\title{
Healthcare quality improvement by redesign
}

\author{
Citation for published version (APA):
}

van Leijen-Zeelenberg, J. E. (2015). Healthcare quality improvement by redesign: aspects of redesigning healtcare processes and the effect on quality of care. [Doctoral Thesis, Maastricht University]. Maastricht University. https://doi.org/10.26481/dis.20151218jl

\section{Document status and date:}

Published: 01/01/2015

DOI:

10.26481/dis.20151218jl

Document Version:

Publisher's PDF, also known as Version of record

\section{Please check the document version of this publication:}

- A submitted manuscript is the version of the article upon submission and before peer-review. There can be important differences between the submitted version and the official published version of record.

People interested in the research are advised to contact the author for the final version of the publication, or visit the DOI to the publisher's website.

- The final author version and the galley proof are versions of the publication after peer review.

- The final published version features the final layout of the paper including the volume, issue and page numbers.

Link to publication

\footnotetext{
General rights rights.

- You may freely distribute the URL identifying the publication in the public portal. please follow below link for the End User Agreement:

www.umlib.nl/taverne-license

Take down policy

If you believe that this document breaches copyright please contact us at:

repository@maastrichtuniversity.nl

providing details and we will investigate your claim.
}

Copyright and moral rights for the publications made accessible in the public portal are retained by the authors and/or other copyright owners and it is a condition of accessing publications that users recognise and abide by the legal requirements associated with these

- Users may download and print one copy of any publication from the public portal for the purpose of private study or research.

- You may not further distribute the material or use it for any profit-making activity or commercial gain

If the publication is distributed under the terms of Article $25 \mathrm{fa}$ of the Dutch Copyright Act, indicated by the "Taverne" license above, 








\section{Healthcare Quality Improvement by Redesign \\ Aspects of redesigning healthcare processes and the effect on quality of care}


The research presented in this thesis was conducted at the School for Public Health and Primary Care (CAPHRI), Department of Health Services Research, Maastricht University.

Printing and dissemination of this thesis was financially supported by CAPHRI RHC program, MUMC+, Department of Otolaryngology and Opera Consultancy \& Implementatie.

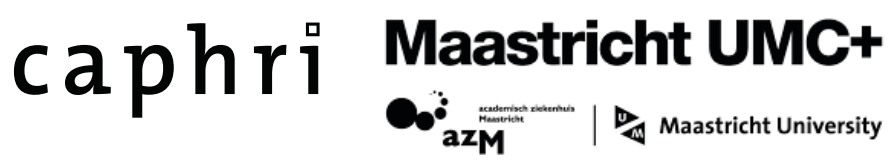

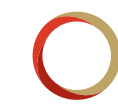

OPERA

Part of the research was performed with the financial support of the Netherlands Organization for Health Research and Development (ZonMw).

(c) Copyright Janneke van Leijen-Zeelenberg Maastricht 2015.

All rights reserved. No part of this thesis may be reproduced or transmitted in any form or by any means, electronic or mechanical, including photocopying, recording, or any information storage or retrieval system without permission from the author, or when appropriate, from the publishers of the publications.

Alle rechten voorbehouden.

Niets uit deze uitgave mag worden verveelvoudigd, opgeslagen in een geautomatiseerd gegevensbestand en/of openbaar gemaakt in enige vorm of op enige wijze, hetzij elektronisch, mechanisch, door fotokopieën, opnamen of op enige andere manier zonder voorafgaande schriftelijke toestemming van de uitgever.

Omslagontwerp: Idske Koning

Vormgeving en druk: PersoonlijkProefschrift.nl, Heleen de Vos

ISBN: 978-90-9029370-7 


\title{
Healthcare Quality Improvement by Redesign
}

\section{Aspects of redesigning healthcare processes and the effect on quality of care}

\author{
PROEFSCHRIFT \\ ter verkrijging van de graad van doctor aan de Universiteit Maastricht, \\ op gezag van de Rector Magnificus, Prof. dr. L.L.G. Soete, \\ volgens het besluit van het College van Decanen, \\ in het openbaar te verdedigen, \\ op vrijdag 18 december 2015 om 14:00 uur
}

door

Janneke Eveline van Leijen-Zeelenberg

Geboren op 17 september 1985 te Benschop 


\section{Promotores}

Prof. dr. D Ruwaard

Prof. dr. B Kremer

Prof. dr. H.J.M. Vrijhoef, Singapore

\section{Co-promotor}

Dr. A.J.A. van Raak

\section{Beoordelingscommissie}

Prof. dr. H.A.M Maarse (voorzitter)

Prof. dr. H.R. Haak

Prof. dr. H.A.M. Marres, Radboud UMC Nijmegen

Prof. dr. G.G. van Merode

Prof. dr. G.P. Westert, Radboud UMC Nijmegen 
Voor mama

'Hoe kostbaar is een kwetsbaar mens' 



\section{CONTENTS}

Chapter $1 \quad$ General Introduction and Aim $\quad 9$

Chapter 2 The Influence of Redesigning Healthcare Processes on the Quality of 27 Care: A Systematic Review

Submitted

Chapter 3 Interprofessional Communication Failures in Acute Care Chains: How 75 Can We Identify the Causes?

Journal of Interprofessional Care, 2015 [epub ahead of print]

Chapter $4 \quad$ Barriers to Implementation of a Redesign of Information Transfer and 97 Feedback in Acute Care: Results From a Multiple Case Study BMC Health Services Research, 2014;14:149.

Chapter 5 Using Lean Thinking Principles at an Otorhinolaryngology Outpatient

Clinic to Improve Patient Care

Laryngoscope, 2015; accepted for publication

Chapter 6 Experiences and Preferences of Patients Visiting an Otolaryngology

Outpatient Clinic: a Qualitative Study

Health Expectations, 2015 [epub ahead of print]

Chapter $7 \quad$ General Discussion and Valorisation

Summary

Nederlandse Samenvatting

Dankwoord

Curriculum Vitae \& List of Publications 



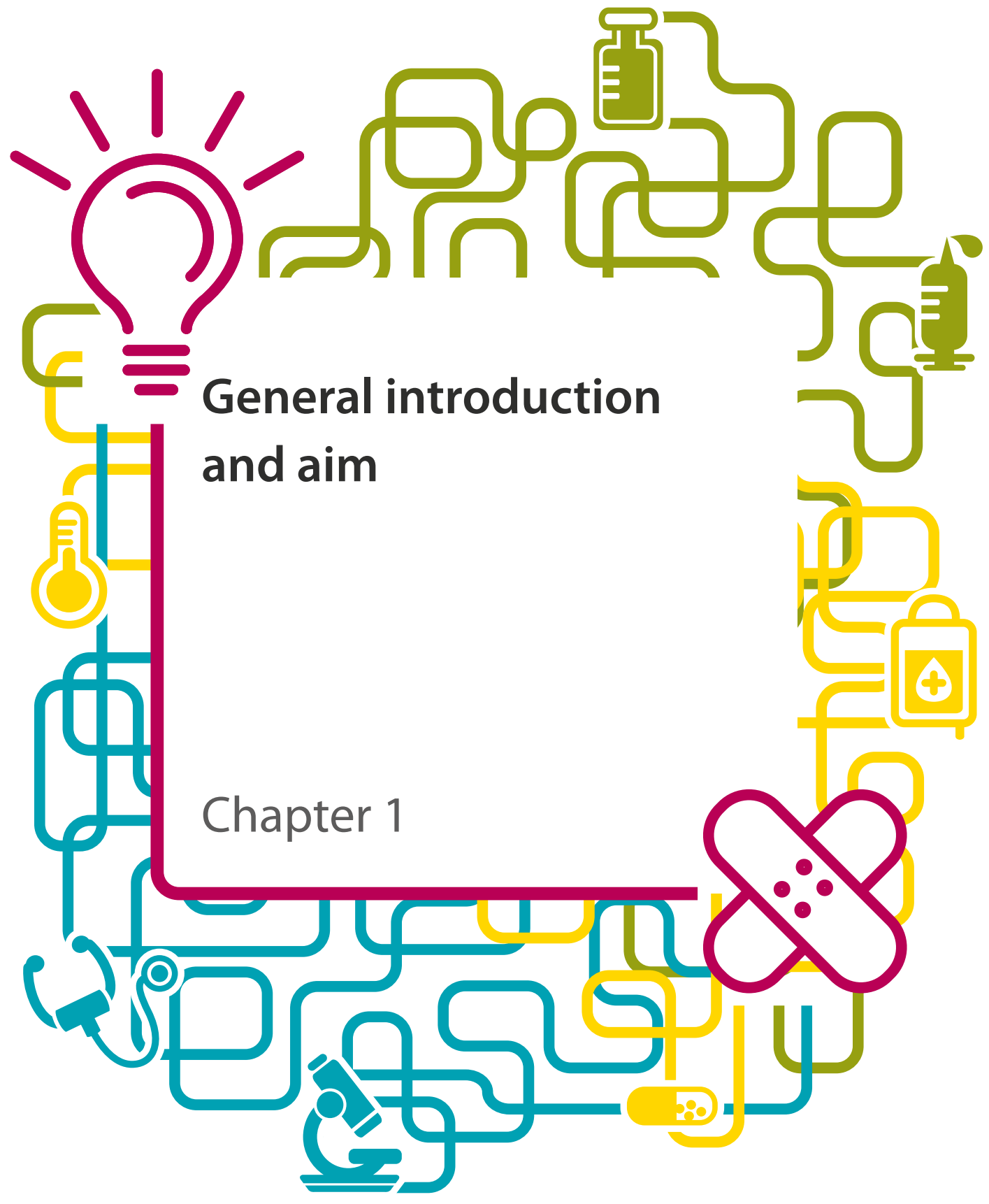


Limitations in achieved quality of care levels and considerable costs are issues in current health systems. Patients do not always receive the right treatments at the right time, and high levels of medical errors - "the failure of a planned action to be completed as intended (i.e., error of execution) or the use of a wrong plan to achieve an aim (i.e., error of planning)"(p.28) ${ }^{1}-$ are still present ${ }^{1-4}$. In addition, increased life expectancy, improved access to care and technological innovation in the last few decades are associated with considerable costs. Alongside these developments, the number of chronically ill patients in the Netherlands is estimated to rise to $40 \%$ of the population in $2030^{5}$, requiring a shift in healthcare service delivery. Self-management, integrated care services, population based health and long-term care facilities are of increased importance ${ }^{5-7}$. These trends can be seen in all Western countries, and are therefore not specific for the Netherlands.

At the same time governments have the tendency to spend less per capita on health care, putting pressure on the health systems across Western countries ${ }^{8,9}$. In the Netherlands, healthcare spending is still rising with an estimated spending of $19 \%$ of the gross domestic product on health care in $2030^{5}$. The growth of care expenditure in the Netherlands is however at its lowest point since $2000^{10}$. Within health care, costs associated with hospital care grow most rapidly as compared to other sectors such as care for the elderly ${ }^{10}$. In order to improve quality of care, to react to the increase of patients with chronical diseases and/or multi morbidities, while keeping care expenditure low, the need to redesign healthcare systems and its processes is internationally acknowledged and many initiatives are presented in literature ${ }^{1,2,11-15}$. An important goal of redesigning healthcare processes is to improve quality of care. Redesign initiatives in health care have shown to lead to improvements such as reduction of waiting times, medical errors and waste. Much of the literature is however descriptive of nature or does not address the need to report how process redesign leads to improved outcomes and in what circumstances ${ }^{2,15}$.

This dissertation reports on two process redesign initiatives in Maastricht, a region in the south of the Netherlands, and explains if, why and how redesign initiatives are successful and which factors contribute to or inhibited its success. This Chapter first describes how quality of care is addressed in this dissertation. The meaning, goals and applications of process redesign and how quality of care is addressed by process redesign is described hereafter. The two process redesign initiatives in the Maastricht region which form the foundation of this dissertation are described and finally, the aims, research methods and outline of the dissertation are presented at the end of this Chapter.

\section{QUALITY OF CARE}

Quality of care is an important topic to clinicians, scientists, policymakers, patients and clients for over decades. Numerous articles have been published and many definitions for quality of care exist. In the Netherlands, making quality of care remunerative for patients, healthcare providers and health insurance companies is emphasized on by the government ${ }^{16}$. An important prerequisite is for all parties involved to understand the definition of quality of care and have access 
to reliable information on quality of care ${ }^{16}$. In 2001, the Institute of Medicine (IoM) published its ground-breaking report 'Crossing the Quality Chasm: A new Health System for the $21^{\text {st }}$ Century' in which important pitfalls in healthcare quality are described and six aims for improvement of healthcare quality are given. This report serves as an important basis for healthcare redesign, as the loM poses that only with fundamental redesign of healthcare systems, high quality of care can be guaranteed. Quality of care is defined by the loM as "the degree to which healthcare services for individuals and populations increase the likelihood of desired outcomes and are consistent with current professional knowledge" ${ }^{11}$. In order to work with this general definition towards improvement, the loM translated the definition into six aims for improvement; health care should be efficient, effective, timely, safe, equitable and patient-centered. The six aims of quality improvement are used as a guideline to define and measure quality of care throughout this thesis. The meaning and application of each aim is explained in this section.

\section{Efficiency}

Efficient healthcare systems are able to use their resources in a way that delivers the best value for the money spent on them'. Value is ultimately defined by the 'customer' and is expressed by a specific product or service which is created by the producer ${ }^{17}$. Opposite to efficiency is waste which can be defined as any activity that does not add value to the customer. In health care, seven types of waste can be defined; (1) transportation - unnecessary movement of patients and employees; (2) inventory - excess stock not being used or patients sitting in the waiting room; (3) motion - any motion that is not necessary for completion of the services, e.g. staff searching for equipment; (4) waiting - idle time between operations, e.g. waiting for results, staff or medicines; (5) overproduction - production of components not for immediate use, e.g. unnecessary diagnostics; (6) over-processing - doing more than necessary, e.g. asking for patients' details several times; (7) defects - steps that need correction, e.g. repeating tests because of errors $^{18}$.

The current healthcare systems are considered to contain large amounts of waste, including overuse and errors that lead to lower quality'. The creation of value adding processes by reducing waste in healthcare systems is therefore considered to lead to a higher level of healthcare quality'.

\section{Effectiveness}

Healthcare effectiveness mainly refers to evidence-based practices ${ }^{1}$. Important components of evidence-based practices are (1) best research evidence - referring to clinically relevant research resulting from four main research types, i.e. laboratory experiments, clinical trials, epidemiological research and outcomes research; (2) clinical expertise - referring to the use of clinical skills and past experience in order to determine the patient's health state, diagnosis risks and benefits of the potential interventions and personal values and expectations; (3) patients values - referring to unique patient preferences, expectations and concerns to be integrated into clinical decision making ${ }^{1}$. As a result of evidence-based practice, patients should only receive 
those interventions or treatments that produce the best outcomes for them and this should be monitored by healthcare organizations. Continuous monitoring should be used to improve one's organization as well as to further knowledge on effective healthcare interventions.

\section{Timeliness}

High quality health care should be delivered on time and the care process should flow smoothly. In the current fragmented healthcare systems, waiting times exist for both patients and providers. Reduction of waiting times is also likely to lead to lower healthcare costs. Several examples and best practices to reduce waiting times exist, also in other industries ${ }^{1}$. The Just-inTime system - an inventory strategy where products or services are only received when needed in the production or delivery process - developed by Taiichi Ohno for the care industry is such an example of minimal waiting times in a production system ${ }^{17}$.

\section{Safety}

In 2000, the loM published its report 'To Err is Human: Building a Safer Health System' in which they made the case for improvement of patient safety ${ }^{19}$. Safety was defined as the absence of accidental injuries and in their report the loM states that health care should be safe for all patients, in all of its processes, all the time. Safety must be enhanced by seamless care processes in which patients only need to tell the caregiver their story once, and where information is accessible, available and correctly transferred to other caregivers. Healthcare organizations should be transparent with regard to their defects and the efforts to continuously improve the organization. According to the loM, a higher level of patient safety in health care will be the first step in improving overall healthcare quality.

\section{Equitable}

High quality health care should be available for all patients in a healthcare system. The health system should improve the health status of a population in that disparities between subgroups are reduced. All patients should have equal access to health care. Next, quality of care should not differ based on characteristics of the individual patients - e.g. gender, age, ethnicity, race, education, disability, sexual orientation, location of residence or income'.

\section{Patient-centeredness}

Health care is patient-centered when it 'delivers care respectful of and responsive to individual patient preferences, needs, values, and ensures that patient values guide all clinical decisions' ${ }^{1}$ p.48). The loM describes six dimensions of patient-centered care (PCC), these being: (1) respect for patients' values, preferences and expressed needs - health care is patient-centered when precisely responding to patient's preferences, needs and wishes; (2) information, communication and education - health care is patient-centered if information on diagnosis, prognosis and treatment is responsive, attentive, trustworthy information tailored to the individual's needs, (3) coordination and integration of care - PCC ensures smooth care transitions to other settings, accurate and timely information transfer to the right persons; (4) emotional support-relieving 
fear and anxiety - PCC reacts to any fear, anxiety or other negative feelings caused by the illness or injury to both the patient and one's family; (5) physical comfort - PCC provides timely and tailored support to experienced shortness of breath, pain or other discomfort of a patient; and (6) involvement of family and friends - In PCC, family and friends of patients are - if appropriate - to be involved in decision making, are supported as caregivers and respected and welcomed in the care delivery setting ${ }^{1}$.

Apart from being a goal in itself, PCC is also considered to lead to improved health outcomes ${ }^{20}$. Applying PCC is considered to lower the costs per patients by reducing the use of diagnostic test, referrals and other health resource utilization, and to improve patient well-being. PCC is also known to lead to increased adherence to treatment and is associated with improved quality of life $\mathrm{e}^{1,20-23}$.

\section{Assessing quality of care}

Once the definition of quality of care is determined, the next challenge is to assess the status of quality of care as delivered. In 1988 Donabedian described a conceptual model to examine healthcare services and to assess quality of care ${ }^{24}$. The model describes three categories of information on quality of care, namely (a) structure - referring to the context in which care is delivered, (b) process - transactions between patients and providers, ranging from prevention to treatment, and (c) outcome - the effect of health care on patients and populations $\mathrm{s}^{24,25}$. Donabedian argues that structure provides the foundation for upright processes which on its term should yield the best health outcomes possible given the present state of health technology. In this dissertation, process redesign initiatives are assessed on their influence of quality of care. Analogously to Donabedian's categories, three questions (i.e. if, why and how) guide the assessment of the redesign initiatives here. Even though Donabedian's work serves as a valuable guide to assessing quality of care, in essence the work described in this dissertation needs to be distinguished here. The work in this dissertation does not attempt to purely assess the state of quality of care, but the effect of process redesign initiatives on quality of care. The model of Donabedian was therefore altered to the specific aims of this dissertation by posing the if, why and how-question. The if-question refers to categories process and outcome, the why-question refers to the categories process and structure and the how-question refers to the category structure of Donabedian's work. The conceptual model used in this dissertation can be found in the aims and outline section of this chapter.

\section{PROCESS REDESIGN}

In order to improve quality of health care, several initiatives are designed in the last few decades. Among others, these initiatives include Total Quality Management (TQM), Business Process Redesign (BPR), Lean Thinking and Six Sigma. Most of these techniques originate from other industries than health care (e.g. the car industry) and the usefulness of these techniques in the healthcare setting remains an issue of debate. Although applying these techniques in healthcare settings has led to improvements, there is still a lack of clear evidence on their effect on quality 
of care - the reasons of which will be described later on in this dissertation ${ }^{12,13,26}$. This section describes the four most known techniques as mentioned earlier in some more detail.

\section{Total Quality Management}

TQM is an approach that aims to improve quality while reducing costs and has been applied to healthcare settings since the late $80^{\prime} \mathrm{s} / 90^{\prime} \mathrm{s}^{27} . \mathrm{TQM}$ is considered to be an organization wide approach incorporating a strategy for personnel and organizational development, a quality management and an informational structure. Not only includes TQM methods for process improvement, it also emphasizes on team methods. TQM is also called continuous quality improvement (CQI) and often defined by four key characteristics of 'good management': (1) empowering clinicians and managers to analyze and improve processes; (2) customer - both patients and providers in health care - preferences are the primary determinant of quality; (3) developing a multidisciplinary approach that exceeds conventional professional lines; and (4) rational and data based cooperative approach to process analysis and change ${ }^{28,29}$. Apart from the four characteristics of good management, the main components of TQM include (1) internal and external customer focus, (2) process analysis, (3) quality project teams, (4) systematic ways of applying simple methods to analyze quality problems, plan changes and evaluate the results, (5) use of data to identify and analyze problems and to evaluate changes, and (6) implementation of change. Implementing TQM in European health care has led to successes, however problems with the implementation of TQM have also occurred ${ }^{27,30}$. The most common barriers towards the implementation of TQM concerned time, sustaining continuous improvements, professional resistance, management resistance and costs of investment without certain returns for the organization ${ }^{27,30}$.

\section{Business Process Redesign}

BPR was developed by industrial staff to implement organizational transformations necessary to shift towards more customer-focused and cost-effective care ${ }^{12,31}$. Although BPR is sometimes also referred to as business process reengineering, both approaches have different starting points. Whereas business process reengineering concerns "the fundamental rethinking and radical redesign of business processes to achieve dramatic improvements in critical contemporary measures of performance, such as cost, quality and speed"32, business process redesign "seeks to improve aspects of the current state while keeping the foundation of the process intact."(p. 115) ${ }^{31}$. BPR-projects are usually carried out in five phase ${ }^{33}$, these being:

1. Planning; identification of project goals, definition of project scope, establishment of project team and development of project plan and schedule;

2. Analysis; full analysis and documentation of the current process also called drawing up the AS IS process. This process is presented and refined until consensus exists. Next, a draft for the redesign of the AS IS process is also created in this phase;

3. Redesign; the AS IS process is reviewed against the improvement goals and improvement opportunities are defined. Next, a new - or adjusted - process is created, also referred to 
as the SHOULD BE process. Complex projects may also include several alternatives in this phase; also referred to as COULD BE processes. Sometimes different COULD BE processes are simulated to facilitate decision making. Finally, the new - or adjusted - process will be described and transition plans will be initiated;

4. Resource development; required resources, space, job descriptions, training, management systems and software systems will be acquired;

5. Transition; this phase concerns the actual implementation of the redesigned process. It is often the hardest phase, since the required change often comes with resistance.

BPR-projects in healthcare settings typically involve multiple interventions and results reported in literature are hard - or even impossible - to compare. Although beneficial outcomes are reported from BPR-projects, most study designs are weak and lack to assign reported outcomes to the implemented intervention components ${ }^{12}$.

\section{Lean Thinking}

Lean Thinking is a method for quality improvement in service delivery that originates from the car industry and is increasingly adopted in health care. Lean Thinking was first applied by Toyota and aims to create value in a system while eliminating waste at the same time. The starting point and first principle of Lean Thinking is to define value to the customer. This definition is not straightforward to obtain and should be carefully defined in terms of specific products or services with specific capabilities and specific customers ${ }^{17}$. Once value is defined, the second principle is to define the value stream map - which is the set of all specific actions required to bring a specific product or service through the business. Most of the times, identifying the value stream map results in the exposure of an amount of waste. The loM uses seven types of waste in defining their concept of efficiency in health care, as described earlier in this Chapter'.

Once the value stream is fully mapped and the waste that was exposed is eliminated, the third principle of Lean Thinking is to create flow. Creating flow requires fundamentally rethinking the organization's structure, in the sense that dividing organization's in departments, functions and careers does not lead to optimal functioning. Rather, focus should lie on making the value flow through the organization. The fourth principle of Lean Thinking is to create a pull system. In a pull system, a product or service is only created then when the client asks for it. Finally, the fifth principle of Lean Thinking is to create a state of perfection. Lean organizations should keep on striving for perfection, as by the creation of flow and pull systems, it is likely that other forms of waste will eventually occur ${ }^{17}$.

Lean can be applied to different healthcare settings, such as outpatient clinics or surgical units. Two recent reviews of literature on the application of Lean Thinking in health care show that thus far, these methodologies are most frequently applied to operation rooms, emergency departments or patient wards ${ }^{11,13}$. In the large majority of these studies, application of Lean led to performance improvements. The implementation of Lean Thinking has shown to lead to 
improved patient satisfaction while enhancing staff morale at the same time, caused by a more efficient use of their expertise ${ }^{34}$.

\section{Six Sigma}

The Six Sigma approach was created by Motorola in the late 1980s and aims to reduce variance and control processes in order to assure high quality performance. Six Sigma produces statistical units reflecting the likelihood that errors will occur and relies on rigorous statistical methods. In essence, Six Sigma seeks to improve processes in such a way that they are consistent within six standard deviations (six sigma's) and thereby only fail in 3.4 out of one million cases. Error reduction in health care has received considerable attention since $2000^{19}$ and Six Sigma has been applied to health care as a means to reduce the amount of avoidable medical errors ${ }^{35}$. Process improvement projects using Six Sigma methods are often guided by a so called champion - a person that is the project sponsor or a member of a steering committee. In most projects, a so called master black belt (who has passed all Six Sigma examinations and has sufficient experience with Six Sigma projects) guides the project. Six Sigma also knows black belts (someone with explicit knowledge of Six Sigma), green belts (with slightly less qualifications of Six Sigma) and sometimes yellow belts (with knowledge of the basic principles of Six Sigma) ${ }^{33}$. Most Six Sigma projects follow the DMAIC circle, which refers to ${ }^{29}$ :

Define - definition of the project, identifying the customer, clarifying the scope, documentation of the project, and definition of project goals.

Measure - identify key measures for the performance of the process, development and testing of data collection methods, compare key measures with customer requirements Analyze - analysis of the existing process, explore possible causes and test hypotheses to identify causes.

Improve - select a solution and pilot test that solution; measure the results and standardize the solutions finally leading to full implementation of the solution.

Control - Control the performance of the process by documenting its performance and keep score of the results through ongoing monitoring.

In health care several barriers to implement Six Sigma exist. First, it can be a challenge to obtain baseline performance data as not all healthcare organizations use optimized monitoring systems. Second, it is often difficult to define processes in health care that can be measured in terms of defects or errors. Third, the statistical and business language of Six Sigma can act as a barrier and fourth, investing in the Six Sigma Belt training system can act as a barrier in health care ${ }^{35}$. If appropriately implemented however, Six Sigma can lead to significant benefits in efficiency, cost-effectiveness and higher process quality in health care ${ }^{11,35-37}$. 


\section{PROCESS REDESIGN IN MAASTRICHT REGION: A TRADITION}

The work described in this dissertation was conducted in two projects. The projects address both acute care delivery as well as elective hospital care. The first project concerns the redesign of information transfer and feedback in acute care chains in the Maastricht region and was carried out by means of a grant by ZonMw. The second project concerns the redesign of the care process of the otorhinolaryngology (ORL) outpatient clinic at the Maastricht UMC+ by means of Lean Thinking. Both projects are embedded in the Research program Redesigning Health Care (RHC) of the CAPHRI Research School (Faculty of Health, Medicine and Life Sciences, Maastricht University) in close cooperation with the Maastricht UMC+ hospital. In the RHC research program, focus lies on interaction and collaboration of different providers working within one or in different settings or echelons in health care. The evaluation of innovative ways to deliver patient- and client-tailored health care (like process redesign) has a long tradition in the RHC program. Early studies in the 1990s addressed quality assurance issues in health care in general ${ }^{38-40}$, and later on studies focused on the effectiveness and evaluation of diverse process innovations (e.g. the implementation and evaluation of disease management programs for chronic diseases ${ }^{41-45}$, integrated care delivery ${ }^{46-55}$, nurse-led healthcare delivery ${ }^{56-59}$, telemonitoring in patients with heart failure ${ }^{60-64}$, evaluating healthcare designs by simulation ${ }^{65-70}$, and optimizing outpatient healthcare delivery ${ }^{71,72}$ ).

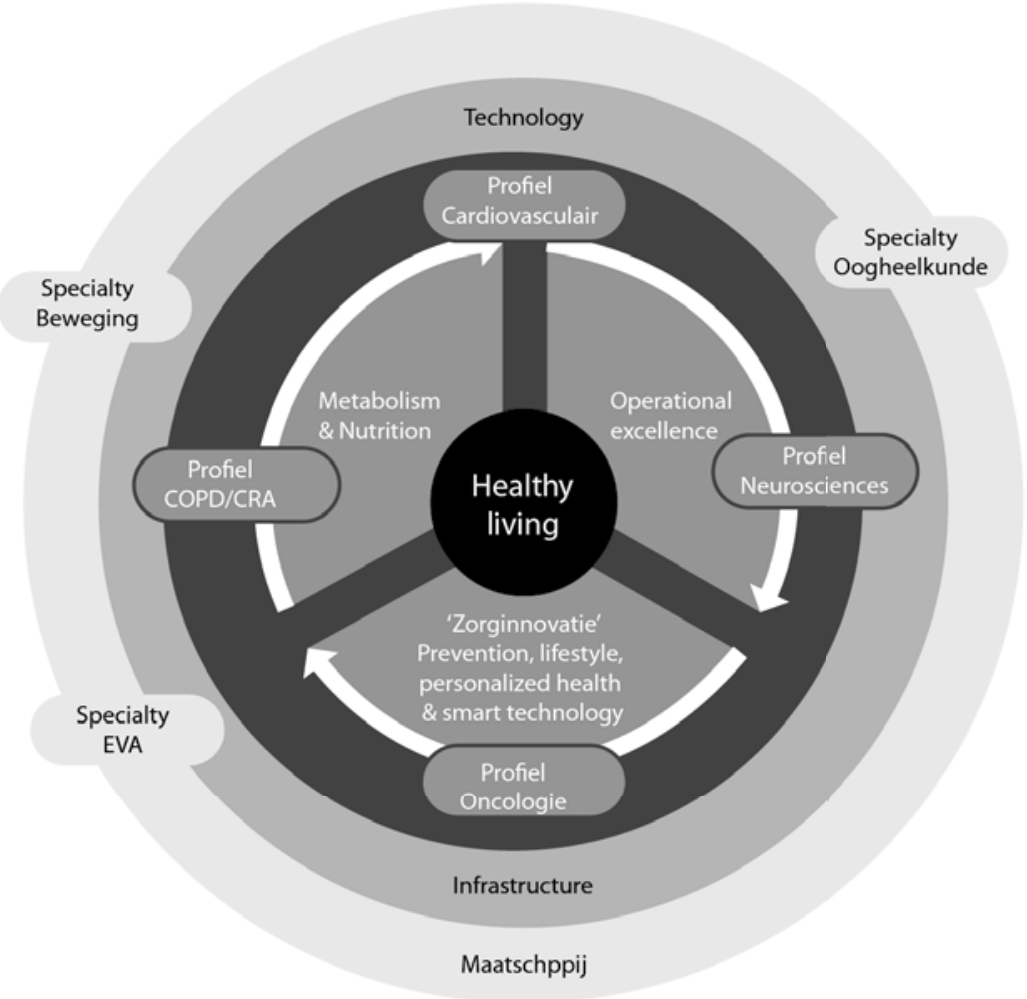

Figure 1 - Adapted from Maastricht Universitair Medisch Centrum. Gezond Leven. Maastricht UMC+ strategie 2020. Maastricht: Maastricht UMC $+; 2015^{73}$ 
The Maastricht UMC+ emphasizes on "delivering the best care possible and to improve the health in the region by integration of patient care delivery, research and education"(p. 1, in Dutch ${ }^{73}$. Ultimately, the Maastricht UMC+ has the ambition to create a state of 'healthy living' in its region ${ }^{74}$. In order to be able to realize and finance this ambition, three transcending innovation themes are emphasized on, these being; Metabolism \& Nutrition, Health Innovation and Operational Excellence (see Figure 1, adapted from Maastricht Universitair Medisch Centrum. Gezond Leven. Maastricht UMC+ strategie 2020. Maastricht: Maastricht UMC+; 2015.). Operational excellence is seen as an organization wide approach and a hard precondition that enables the Maastricht UMC+ to realize its ambition. By 2020, the organization should breath operational excellence and the system approach should no longer be present anymore ${ }^{73}$. The research conducted for this dissertation closely relates to the ambition of the Maastricht UMC+, as it executes research in the field of operational excellence (i.e. improving value for the customer and reduction of waste).

The projects carried out for this dissertation are therefore well embedded both within the RHC research program and the Maastricht UMC+.

\section{Redesign of information transfer and feedback in acute care chains}

In the Netherlands, acute care is increasingly organized in care chains with multiple care providers, with the aim to provide seamless care to patients with acute care needs ${ }^{75}$. Healthcare providers in these acute care chains indicated that communication failures exist, leading to unwanted situations in care provision ${ }^{76}$. In order to improve information transfer in these acute care chains, the redesign project aimed to understand the root causes of the communication failures and consecutively to implement a model for information transfer and feedback. Together with understanding the root causes of communication failures, evaluating the barriers and success factors for implementation of the redesign were the central aims of this study. The research project was carried out between October 2009 and April 2011 by means of a grant from ZonMw (nr. 82711006) and the project was entitled "Improvement of communication and information transfer in acute care chains by implementation of a model for information transfer and feedback".

\section{Redesigning the primary care process at an otorhinolaryngology outpatient clinic}

Between September 2011 and April 2013, a project which aimed to increase efficiency of the ORL outpatient clinic of Maastricht UMC+ hospital was carried out. The ORL outpatient clinic is a tertiary outpatient clinic with both an academic and a regional function. In 2011 the outpatient clinic formulated the mission to create the ideal outpatient clinic for both patients and the employees. In this outpatient clinic the patient knows beforehand what to expect when visiting the outpatient clinic, and after his visit, the expectations are met or even exceeded. This mission was formulated at the kick-off of the redesign project. The main goals of the project were to detect and reduce waste at the outpatient clinic and to improve both patient and provider satisfaction. For this end, Lean Thinking principles were implemented and its effects on waste, patient satisfaction and provider satisfaction were evaluated. The success factors and barriers 
for implementation was evaluated and, finally, patients' experiences with and preferences for care provided at the outpatient clinic were explored in this study.

\section{AIM AND OUTLINE}

\section{Aim and research methods}

The overall aim of this dissertation is to explore if, why and how redesign initiatives are successful and which factors contribute to or inhibit the success of process redesign. In more detail, the studies in this dissertation describe:

- The effects of the redesign initiatives on several dimensions of quality of care (i.e. if the redesign works);

- Factors contributing to the success or failure of the redesign initiative (i.e. why the redesign works or not); and

- The effect of the redesign initiative on other aspects of the organizations (i.e. how the redesign works).

Together, these three questions form the framework for the analysis of the redesign initiatives (Figure 2). The questions of the framework are applied to both the acute and elective care setting.

\section{Evaluating process redesign \\ initiatives in health care}



The IF-question:

What is the effect of

the redesign initiative on quality of care?

- Effectiveness

- Efficiency

- Timeliness

- Patient-centeredness

- Safety

- Equity

The WHY-question:

Why did the

redesign initiative

work or not?

- Facilitators

- Barriers

- Sense of Urgency
The HOW-question:

How did the redesign

initiative affect the

organization?

- Willingness to

change

- Openness

- Feedback Culture

Figure 2 - Conceptual framework for this dissertation

As stated earlier, the framework presented here uses an alteration of Donabedian's three categories ${ }^{24}$ to structure the research aims and questions. In contrast to Donabedian's framework, the if-question (outcome) is first addressed here, after which the why (process and structure) and how-question (structure) are addressed. The underlying hypothesis used in this dissertation is that process redesign initiatives not only affect quality of care (outcomes), but also affect process and structure of the organizations involved. We believe this understanding is necessary to oversee the full impact of redesign initiatives and hence goes beyond understanding the 
impact of redesign initiatives on outcomes alone.

In both projects participatory action research (PAR) is used as main research method. The main difference between PAR and more classic designs of research is the active involvement of people who are affected by the subject under study ${ }^{77}$. This means that a project group containing both researchers and end-users of the redesign initiatives is responsible for the implementation of the redesign intervention in the projects and that the evaluation of the projects also involves collaboration between the researcher and the end-users of the redesigns. The researcher therefore takes a participatory role, leading to a situation where study objects and the researcher jointly work towards improvement and learn from each other. An important feature of this type of research is its ability to bridge the gap between research and practice, by emphasizing on characteristics like culture, settings and people rather than on effectiveness alone ${ }^{78}$. Using PAR as a research strategy often involves using mixed methods for data collection, as is done in this dissertation. Using mixed methods enables researchers to triangulate data, leading to deeper understandings of the subject under issue ${ }^{79,80}$. Semi-structured interviews, focus groups, questionnaires, collection of physical artifacts and archival records are the main sources of data collection in this dissertation. The use of these techniques is applied and mixed differently throughout the projects. In order to understand the root causes of communication failures in acute care chains (addressing the why-question), semi-structured interviews, collection of physical artifacts (describing the communication process) and archival records (on communication failures and process descriptions) are used and triangulated. Barriers for the implementation of a communication redesign initiative (addressing the why-question) are assessed by using focus group interviews to deepen the findings of a questionnaire. The effects of Lean implementation at an ORL outpatient clinic (addressing if and how-question) are described using semi-structured interviews to understand the effects on waste (if) and organizational culture (how) and questionnaires to measure changes in patient satisfaction (if) and provider satisfaction (how). Finally, a focus group is used to verify and deepen the findings on patients' expectations and preferences retrieved by semi-structured interviews for ORL outpatient care (if).

\section{Dissertation outline}

The aim of this dissertation is elaborated on in different Chapters of this dissertation. Chapter 2 focuses on the influence of process redesign on quality of care in general by means of a systematic review of the available literature. In Chapter 3 root causes for communication failures in acute care chains are explored, using theory on organization characteristics and organizational routines as a perspective. Chapter 4 describes barriers for the implementation of a redesign for information transfer and feedback in an acute care setting. In Chapter 5, the effect of lean implementation on patient and provider satisfaction, waste reduction and providers' perspectives on cultural change at an ORL outpatient clinic is described. Chapter 6 describes an in-depth evaluation of patients' perspectives on PCC in an ORL outpatient clinic. In Chapter 7 the main findings of this dissertation are summarized, methodological consideration and societal impact of these findings are discussed and recommendations are presented. 


\section{REFERENCES}

1. Institute of Medicine. Crossing the Quality Chasm: A New Health System for the 21st Century. Washington, DC: National Academies Press; 2001.

2. Locock L. Healthcare redesign: meaning, origins and application. Qual Saf Health Care. 2003;12(1):53-57.

3. Leape LL, Berwick DM. Five years after To Err Is Human: what have we learned? Jama. 2005;293(19):2384-2390.

4. Stelfox HT, Palmisani S, Scurlock C, Orav EJ, Bates DW. The "To Err is Human" report and the patient safety literature. Qual Saf Health Care. 2006;15(3):174-178.

5. Rijksinstituut voor Volksgezondheid en Milieu. Een gezonder Nederland. Volksgezondheid Toekomst Verkenning 2014. Bilthoven: RIVM;2014.

6. Directorate-General for Research and Innovation. Horizon 2020. Work programme 2014-2015. Health, Demographic change and wellbeing. Brussels: European Commission;2013.

7. World Health Organization. People-centered and integrated health services: an overview of the evidence. Interim Report. Geneva: World Health Organization;2015.

8. OECD. Health at a Glance: Europe 2012. Paris: The Organisation for Economic Co-operation and Development; 2012.

9. Raad voor de Volksgezondheid en Zorg. Perspectief op gezondheid 20/20. Den Haag; 2010.

10. Centraal Bureau voor de Statistiek. CBS: growth care expenditure at lowest level in 15 years. Den Haag; 2013.

11. DelliFraine JL, Langabeer JR, 2nd, Nembhard IM. Assessing the evidence of Six Sigma and Lean in the health care industry. Qual Manag Health Care. Sep 2010;19(3):211-225.

12. Elkhuizen SG, Limburg M, Bakker PJ, Klazinga NS. Evidence-based re-engineering: re-engineering the evidence--a systematic review of the literature on business process redesign (BPR) in hospital care. Int J Health Care Qual Assur Inc Leadersh Health Serv. 2006;19(6-7):477-499.

13. Mazzocato P, Savage C, Brommels M, Aronsson H, Thor J. Lean thinking in healthcare: a realist review of the literature. Qual Saf Health Care. 2010;19(5):376-382.

14. Radnor ZJ, Holweg M, Waring J. Lean in healthcare: the unfilled promise? Soc Sci Med. 2012;74(3):364-371.

15. Walshe K, Freeman T. Effectiveness of quality improvement: learning from evaluations. Qual Saf Health Care. 2002;11(1):85-87.

16. Schippers El. Het jaar van de transparantie. Den Haag: Ministerie van Volksgezondheid Welzijn en Sport; 2015; 723160-133094-MC.

17. Womack J, Jones D. Lean Thinking. Banish waste and create wealth in you corporation. New york: Free Press; 2003. 
18. Tapping D, Luyster T, Shuker T. Value Stream Management. Eigt steps to planning, mapping, and sustaining lean improvements. New York: Productivity Press; 2002.

19. Institute of Medicine. To Err is Human: Building a Safer Health System. Washington D.C.: Institute of Medicine; 2000.

20. De Boer D, Delnoij D, Rademakers J. The importance of patient-centered care for various patient groups. Patient Educ Couns. 2013;90(3):405-410.

21. Bauman AE, Fardy HJ, Harris PG. Getting it right: why bother with patient-centred care? Med J Aust. 2003;179(5):253-256.

22. Bertakis KD, Azari R. Patient-centered care is associated with decreased health care utilization. JAm Board Fam Med. 2011;24(3):229-239.

23. Hunter R, Carlson E. Finding the fit: patient-centered care. Nurs Manage. 2014;45(1):38-43.

24. Donabedian A. The Quality of Care - How Can It Be Assessed. Jama-J Am Med Assoc. 1988;260(12):1743-1748.

25. Donabedian A. An introduction to Quality Assurance in Health Care. Orford: University Press; 2003.

26. Schouten LM, Hulscher ME, van Everdingen JJ, Huijsman R, Grol RP. Evidence for the impact of quality improvement collaboratives: systematic review. BMJ. 2008;336(7659):1491-1494.

27. Ovretveit J. Total quality management in European healthcare. Int J Health Care Qual Assur. 2000;13(2):74-79.

28. Kaluzny AD, McLaughlin CP, Simpson K. Applying total quality management concepts to public health organizations. Public Health Rep. 1992;107(3):257-264.

29. McLaughlin CP, Kaluzny AD. Total quality management in health: making it work. Health Care Manage Rev. 1990;15(3):7-14.

30. Mosadeghrad AM. Obstacles to TQM success in health care systems. Int J Health Care Qual Assur. 2013;26(2):147-173.

31. Bohn A, Te SA. Process Redesign in Healthcare. In: Larson JA, ed. Management Engineering. A Guide to Best Practices for Industrial Engineering in Health Care. Boca Raton: CRC Press, Taylor \& Francis Group, LLC; 2014.

32. Hammer M, Champy J. Reengineering the corporation: A manifesto for business revolution. Business Horizons. 1993;36(5):90-91.

33. Harmon P. Business Process Change. A manager's guide to improving, redesigning and automating processes. San Francisco, CA: Morgan Kaufmann Publishers; 2003.

34. Dickson EW, Anguelov Z, Vetterick D, Eller A, Singh S. Use of lean in the emergency department: a case series of 4 hospitals. Ann Emerg Med. 2009;54(4):504-510.

35. Taner M, Sezen B, Antony J. An overview of Six Sigma applications in healthcare Industry. Int J Health Care Qual Assur Inc Leadersh Health Serv. 2007;20(4):329-340. 
36. DelliFraine JL, Wang Z, McCaughey D, Langabeer JR, 2nd, Erwin CO. The use of six sigma in health care management: are we using it to its full potential? Qual Manag Health Care. 2013;22(3):210-223.

37. Feng $\mathrm{Q}$, Manuel $\mathrm{CM}$. Under the knife: a national survey of six sigma programs in US healthcare organizations. International journal of health care quality assurance. 2008;21(6):535-547.

38. Harteloh PP, Verheggen FW. Quality assurance in health care. From a traditional towards a modern approach. Health Policy. 1994;27(3):261-270.

39. Verheggen FW, Harteloh PP. [Service quality in health care: the application of the results of marketing research]. Salud Publica Mex. 1993;35(3):316-320.

40. Verheggen FW, Van Wijmen FC. [The effectiveness of quality assurance committees in a university hospital]. Salud Publica Mex. 1993;35(3):311-315.

41. Steuten L, Lemmens K, Vrijhoef B. Health technology assessment of asthma disease management programs. Curr Opin Allergy Clin Immunol. 2007;7(3):242-248.

42. Steuten L, Palmer S, Vrijhoef B, van Merode F, Spreeuwenberg C, Severens H. Cost-utility of a disease management program for patients with asthma. Int JTechnol Assess Health Care. 2007;23(2):184-191.

43. Steuten LM, Vrijhoef HJ, Landewe-Cleuren S, Schaper N, Van Merode GG, Spreeuwenberg C. A disease management programme for patients with diabetes mellitus is associated with improved quality of care within existing budgets. Diabet Med. 2007;24(10):1112-1120.

44. Steuten LM, Vrijhoef HJ, Spreeuwenberg C, Van Merode GG. Participation of general practitioners in disease management: experiences from The Netherlands. Int J Integr Care. 2002;2:e24.

45. Vrijhoef HJ, Spreeuwenberg C, Eijkelberg IM, Wolffenbuttel BH, van Merode GG. Adoption of disease management model for diabetes in region of Maastricht. BMJ. 2001;323(7319):983-985.

46. Paulus AT, van Raak A, Keijzer F. Informal and formal caregivers' involvement in nursing home care activities: impact of integrated care. J Adv Nurs. 2005;49(4):354-366.

47. Paulus AT, van Raak AJ. The impact of integrated care on direct nursing home care. Health Policy. 2008;85(1):45-59.

48. Paulus AT, van Raak AJ, Maarse HJ. Is integrated nursing home care cheaper than traditional care? A cost comparison. Int J Nurs Stud. 2008;45(12):1764-1777.

49. Van Raak A, Groothuis S, van der Aa R, Limburg M, Vos L. Shifting stroke care from the hospital to the nursing home: explaining the outcomes of a Dutch case. J Eval Clin Pract. 2010;16(6):1203-1208.

50. Van Raak A, Meijer E, Meijer A, Paulus A. Sustainable partnerships for integrated care: the role of decision making and its environment. Int $J$ Health Plann Manage. 2005;20(2):159-180.

51. Van Raak A, Mur-Veeman I, Paulus A. Understanding the feasibility of integrated care: a rival viewpoint on the influence of actions and the institutional context. Int $J$ Health Plann Manage. 1999;14(3):235-248.

52. Van Raak A, Paulus A, Cuijpers R, Te Velde C. Problems of integrated palliative care: a Dutch case study of routines and cooperation in the region of Arnhem. Health Place. 2008;14(4):768-778. 
53. Van Raak A, Paulus A, Cuijpers R, te Velde C. Problems of integrated palliative care: a Dutch case study of routines and cooperation in the region of Arnhem. Health Place. 2008;14:768-778.

54. Van Raak A, Paulus A, van Merode F, Mur-Veeman I. Integrated care management: applying control theory to networks. J Manag Med. 1999;13(6):390-404.

55. Van Raak A, Paulus ATG, Groothuis S. Integrated Care Delivery: Process Redesign and the Role of Rules, Routines and Transaction Costs. In: Klein LA, Neumann EL, eds. Integrated Heatlh Care Delivers. New York: Nova Science Publishers; 2008:115-135.

56. Hendriks JL, Nieuwlaat R, Vrijhoef HJ, de Wit R, Crijns HJ, Tieleman RG. Improving guideline adherence in the treatment of atrial fibrillation by implementing an integrated chronic care program. Neth Heart J. 2010;18(10):471-477.

57. Hendriks JM, de Wit R, Crijns HJ, et al. Nurse-led care vs. usual care for patients with atrial fibrillation: results of a randomized trial of integrated chronic care vs. routine clinical care in ambulatory patients with atrial fibrillation. Eur Heart J. 2012;33(21):2692-2699.

58. Hendriks JM, de Wit R, Vrijhoef HJ, Tieleman RG, Crijns HJ. An integrated chronic care program for patients with atrial fibrillation: study protocol and methodology for an ongoing prospective randomised controlled trial. Int J Nurs Stud. 2010;47(10):1310-1316.

59. Hendriks JM, Vrijhoef HJ, Crijns HJ, Brunner-La Rocca HP. The effect of a nurse-led integrated chronic care approach on quality of life in patients with atrial fibrillation. Europace. 2014;16(4):491-499.

60. Boyne JJ, Vrijhoef HJ, Wit R, Gorgels AP. Telemonitoring in patients with heart failure, the TEHAF study: Study protocol of an ongoing prospective randomised trial. Int J Nurs Stud. 2011;48(1):94-99.

61. Boyne JJ, Vrijhoef HJ, Spreeuwenberg M, De Weerd G, Kragten J, Gorgels AP. Effects of tailored telemonitoring on heart failure patients' knowledge, self-care, self-efficacy and adherence: a randomized controlled trial. Eur J Cardiovasc Nurs. 2014;13(3):243-252.

62. Boyne JJ, Vrijhoef HJ, Crijns HJ, De Weerd G, Kragten J, Gorgels AP. Tailored telemonitoring in patients with heart failure: results of a multicentre randomized controlled trial. Eur J Heart Fail. 2012;14(7):791-801.

63. Boyne JJ, Vrijhoef $\mathrm{HJ}$. Implementing telemonitoring in heart failure care: barriers from the perspectives of patients, healthcare professionals and healthcare organizations. Curr Heart Fail Rep. 2013;10(3):254-261.

64. Boyne JJ, Van Asselt AD, Gorgels AP, et al. Cost-effectiveness analysis of telemonitoring versus usual care in patients with heart failure: the TEHAF-study. J Telemed Telecare. 2013;19(5):242-248.

65. Groothuis S, Goldschmidt HM, Drupsteen EJ, de Vries JC, Hasman A, van Merode GG. Application of computer simulation analysis to assess the effects of relocating a hospital phlebotomy department. Ann Clin Biochem. 2002;39(Pt 3):261-272.

66. Groothuis S, Goldschmidt HM, Drupsteen EJ, de Vries JC, Hasman A, van Merode GG. Turn-around time for chemical and endocrinology analyzers studied using simulation. Clin Chem Lab Med. 2002;40(2):174-181. 
67. Groothuis S, Hasman A, van Pol PE, et al. Predicting capacities required in cardiology units for heart failure patients via simulation. Comput Methods Programs Biomed. 2004;74(2):129-141.

68. Groothuis S, van Merode GG. Discrete event simulation in the health policy and management program. Methods Inf Med. 2000;39(4-5):339-342.

69. Molema JJ, Groothuis S, Baars IJ, et al. Healthcare system design and parttime working doctors. Health Care Manag Sci. 2007;10(4):365-371.

70. van Merode GG, Groothuis S, Schoenmakers M, Boersma HH. Simulation studies and the alignment of interests. Health Care Manag Sci. 2002;5(2):97-102.

71. Rouppe van der Voort MM, van Merode FG, Berden BH. Making sense of delays in outpatient specialty care: a system perspective. Health Policy. 2010;97(1):44-52.

72. Rouppe van der Voort MM, van Wijngaarden J, Janssen S, Berden B, van Merode F. Sustainability of improvements in access to outpatient specialist care in the Netherlands. J Health Serv Res Policy. 2014;19(2):94-101.

73. Maastricht Universitair Medisch Centrum. Gezond Leven. Maastricht UMC+ strategie 2020. Maastricht: Maastricht UMC+; 2015.

74. Maastricht Universitair Medisch Centrum. Better health in a changing world. Maastricht: Maastricht UMC+; 2012.

75. Gijsen R, Kommer GJ, Bos N, van Stel H. Hoe is acute zorg georganiseerd? In: Volksgezondheid Toekomst Verkenning, Nationaal Kompas Volksgezondheid 2010; retrieved from:

76. http://www.nationaalkompas.nl/zorg/acute-zorg/hoe-is-de-acute-zorg-georganiseerd/, 2010

77. Traumacentrum Limburg. Failure Mode and Effects Analysis CVA, Myocardinfarct, Heuptrauma, Obstetrie, Psychiatrie. Maastricht: Traumacentrum Limburg; 2009.

78. Cornwall A, Jewkes R. What is participatory research? Soc Sci Med. 1995;41(12):1667-1676.

79. Cargo M, Mercer SL. The value and challenges of participatory research: strengthening its practice. Annu Rev Public Health. 2008;29:325-350.

80. Halcomb E, Hickman L. Mixed methods research. Nurs Stand. 2015;29(32):41-47.

81. Creswell JW PCV. Choosing a mixed methods design. Designing and conducting mixed methods research. Thousand Oaks, California: 



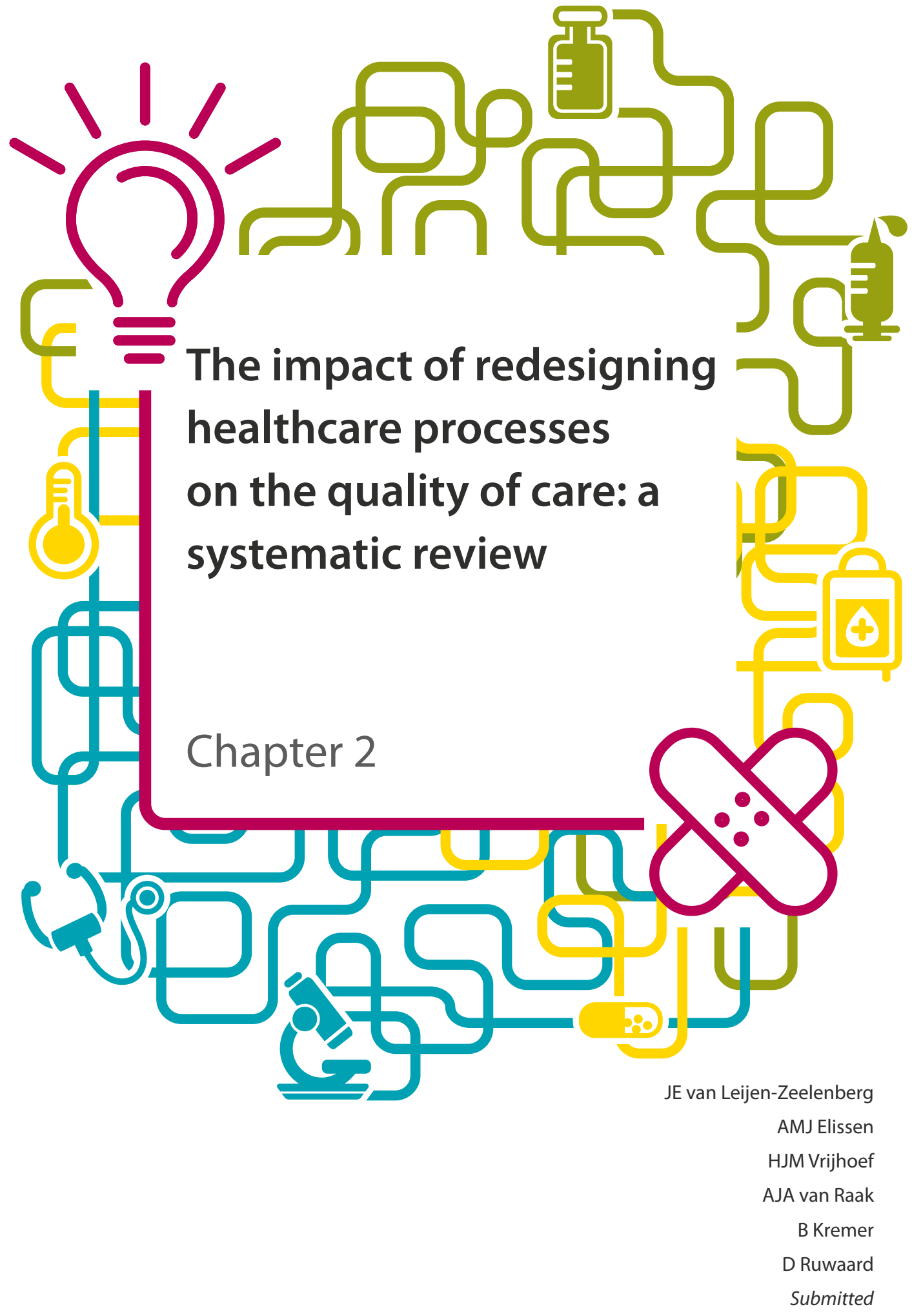




\section{ABSTRACT}

\section{Background:}

This literature review evaluates the current state of knowledge about the impact of process redesign on the quality of healthcare.

\section{Methods:}

Pubmed, CINAHL, Web of Science and Business Premier Source were searched for relevant studies published in the last ten years [2004-2014]. To be included, studies had to be original research, published in English with a before-and-after study design, and be focused on changes in healthcare processes and quality of care. Studies that met the inclusion criteria were independently assessed for excellence in reporting by three reviewers using the SQUIRE checklist. Data was extracted using a framework developed for this review.

\section{Results:}

Reporting adequacy varied across the studies. Process redesign interventions were diverse, and none of the studies described their effects on all dimensions of quality defined by the Institute of Medicine.

\section{Conclusions:}

The influence of process redesign on quality of care could not be established, as none of the articles reported on all its aspects. A wide range of outcome measures were used, and research methods were limited. This review demonstrates the need for further investigation of the impact of redesign interventions on the quality of healthcare.

\section{Key Words:}

Process redesign, quality of care, healthcare processes, systematic review 


\section{INTRODUCTION}

Growing expenditure on healthcare and ongoing efforts to improve services give impetus to change in processes and systems ${ }^{1}$. As life expectancy increases, so does chronic disease, which is associated with a greater demand for multidisciplinary care ${ }^{2,3}$. At the same time, public outlay on healthcare has decreased, inducing potential shortages of healthcare providers ${ }^{3}$. Long-term implications for the quality of care are unclear and should be carefully monitored ${ }^{3}$. According to the Institute of Medicine (IoM), patients do not always receive the most suitable care, at the best time or the best place 2 . Its influential report 'Crossing the Quality Chasm: A New Health System for the $21^{\text {st }}$ Century' emphasized the need to redesign healthcare processes and systems in response to this quality gap. It called upon providers to ensure more efficient, safe, timely, effective, patient-centered and equitable care ${ }^{2,4}$.

Although some initiatives were undertaken before 2001, the publication of the loM report served as a catalyst ${ }^{2,5}$. Numerous interventions - disease management programs for the chronically ill, quality improvement collaboratives, and change programs - are tested and implemented annually on different scales and within different setting $\mathrm{s}^{5}$. Nonetheless, progress is slow; evaluations of initiatives are inconsistent and available knowledge fragmented ${ }^{5}$. The effects are not homogeneous and the research designs used to measure them are generally weak $k^{4,6,7}$.

This study seeks to establish, through a review of the literature, what is known about the influence of redesigning healthcare processes on the quality of care delivered in the last ten years. Its specific aims are to report (a) the content of the interventions (their objectives and implementation methods); (b) the characteristics of the redesign investigations (study design and setting); and (c) the outcomes on quality of care (patient safety, effectiveness, efficiency, patient-centeredness, timeliness, and equitability). The objective of this literature review is to summarize the current state of knowledge on redesigning healthcare processes and present an overview of improvement efforts in the field.

The review applies several key concepts. The first is 'process redesign', defined as any methodology that focuses on creating new processes or changing existing ones in major ways ${ }^{8}$. That definition is deliberately broad so as to cover as many interventions as possible; recourse to dedicated design concepts - such as 'lean thinking', 'business process re-engineering' or 'six sigma' - might exclude relevant studies. The second is 'quality of care', connoting healthcare that is safe, effective, patient-centered, timely, efficient and equitable ${ }^{2}$. The third is 'healthcare processes', defined as "the activities that constitute healthcare - including diagnosis, treatment, rehabilitation, prevention, and patient education - usually carried out by professional personnel, but also including other contributions to care, particularly by patients and their families"(p. 46).

\section{METHODS}

\section{Information sources and search strategy}

The search strategy was guided by the PRISMA statement ${ }^{10}$. It was designed to access published work and comprised two stages: 
1. An extensive search in Pubmed, CINAHL, Business Source Premier and Web of Science, using predefined search terms and free-text words;

2. A search of the reference lists in the included full-text articles.

From March through April 2014, the databases PubMed, CINAHL, Web of Science and Business Premier Source (EBSCO-host) were searched by one reviewer (JvL). In PubMed, MeSH terms were used; CINAHL Heading terms were used for CINAHL; and Thesaurus terms were used for Business Premier Source. For Web of Science no predefined keywords were available. Additionally, free-text words were used for all databases. An overview of the search terms is given in Appendix 1.

The database search was limited to articles published in English between January 2004 and April 2014. Articles were included if they presented original research on redesign of healthcare processes, quality of care, and if they assessed the same outcome measures before and after an intervention. (See Table 1 for inclusion and exclusion criteria). Three reviewers (JvL, KG \& AE) independently screened titles and abstracts for relevance. The reviewers then held a consensus meeting on the inclusion of articles. When that did not yield agreement, the full text was reviewed and discussed to arrive at a decision. Subsequently, reference lists and bibliographies of all included full-text articles from the first stage were searched for additional studies.

Table 1 - Inclusion and exclusion criteria

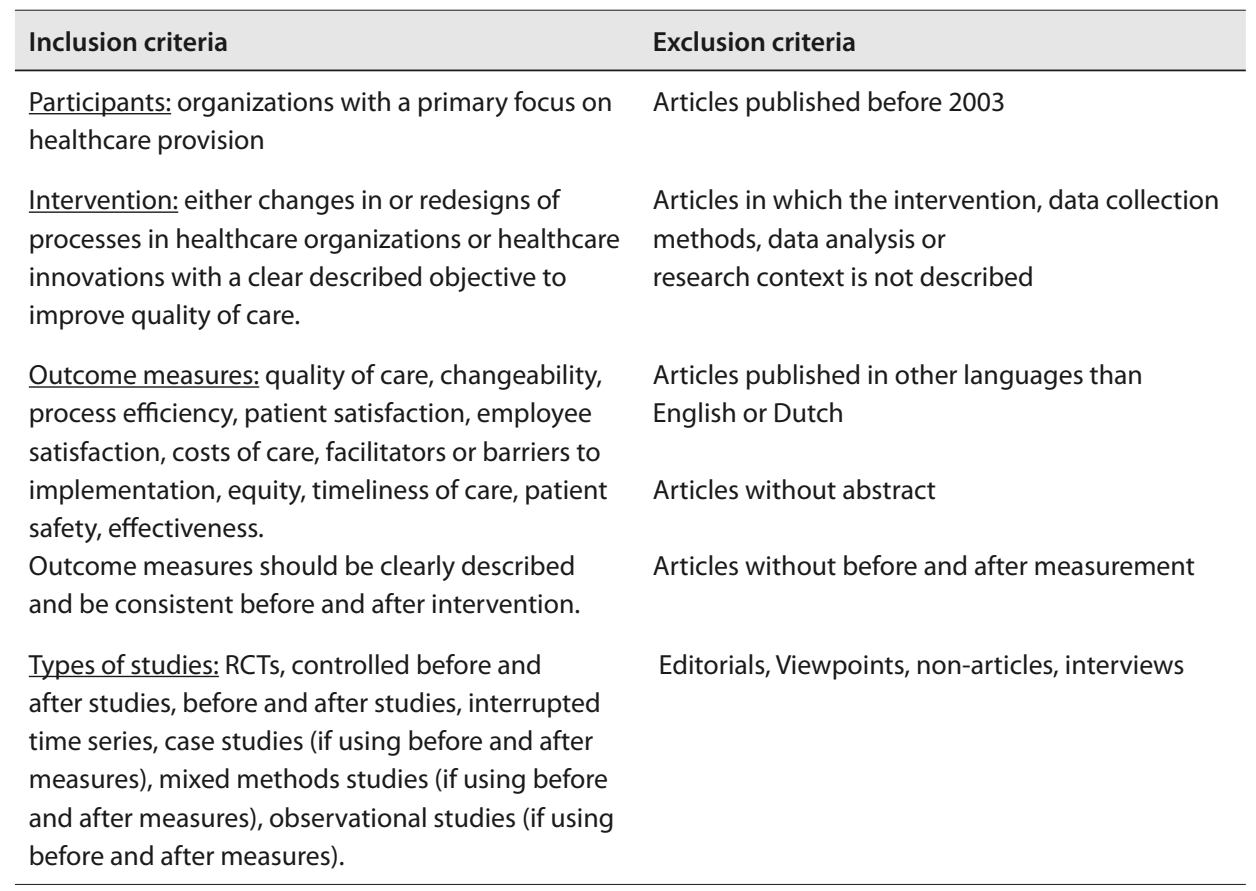




\section{Critical appraisal}

Studies meeting the criteria were assessed independently for reporting excellence by three reviewers ( $J \mathrm{VL}, \mathrm{AE} \& \mathrm{KG}$ ) prior to inclusion in light of the Standards for Quality Improvement Reporting Excellence (SQUIRE). That checklist provides guidelines for reporting of studies assessing the effectiveness of interventions to improve quality and safety of care. Its 19 items comprise 38 components ${ }^{11}$. Any disagreements between reviewers were resolved through consensus.

\section{Data extraction and analysis}

After compliance with the reporting guidelines had been assessed, data were extracted independently by three reviewers ( $\mathrm{JL}, \mathrm{KG} \& \mathrm{AE}$ ) from the results and discussion/conclusion sections. For that purpose, a framework was developed; the form contained variables such as publication year, study objectives, characteristics of the redesign and outcome measures. Any disagreements were resolved through consensus. Meta-analysis could not be performed because the studies used different outcome measures and research designs.

\section{RESULTS}

Figure 1 shows the steps leading to inclusion in the review. Initially, after removing duplicates $(\mathrm{N}=27), 451$ articles were found in the first stage, 11 of which were then included on the basis of their titles and abstracts. Perusal of their reference lists yielded another 24 articles for screening of title and abstract. Based on titles and abstracts, 21 articles were assessed for eligibility. On eight of these, consensus was only reached after reviewing the full text. After assessing the reporting excellence, three articles were excluded. One was removed because it did not describe data collection and timepoints, so it could not be determined whether a before-and-after measurement was performed. Another was removed because it was unclear whether it concerned original research; moreover, the main intervention (presence of a nurse coordinator) did not qualify as process redesign. The third was removed because it was unclear whether the intervention was actually implemented and whether before-and-after measurement was carried out but also because the outcome measures differed at various timepoints.

\section{Reporting Excellence}

Table 2 summarizes the findings according to SQUIRE guidelines. The number of components described range from $11^{12}$ to $27^{13}$, with most articles reporting on 20 or more ${ }^{13-22}$. Overall, methods of evaluation and analysis are the least well described. The majority described the research setting $(\mathrm{N}=16)^{12-27}$, intervention components and parts $(\mathrm{N}=16)^{13-16,18-28}$, main factors in the choice of intervention $(\mathrm{N}=15)^{12-18,20,22-28}$, and primary and secondary outcomes $(\mathrm{N}=15)^{12-14,16-}$ $24,28,29$. Thirteen articles presented evidence on the strength of the association between the intervention and changes observed $(\mathrm{N}=13)^{12,13,16-22,24,25,27-29}$. Half gave details on the qualitative and quantitative methods applied $(\mathrm{N}=9)^{13,17-20,24,25,28,29}$ or aligned the unit of analysis with the intervention $(\mathrm{N}=9)^{13-15,18-21,24,28}$. Six described internal and external validity $13,15,17-20,28$, whereas two dealt with the validity and reliability of instruments ${ }^{17,28}$. Whereas none of the articles explicitly stated 
the study questions, all of them specified the aims of the intervention. Most data concerned changes observed in the care delivery process $(\mathrm{N}=12)^{13-15,18,21-24,26,28,29}$ or differences in patient outcomes $(\mathrm{N}=12)^{13,16-24,28,29}$.



Figure 1 - Search strategy 


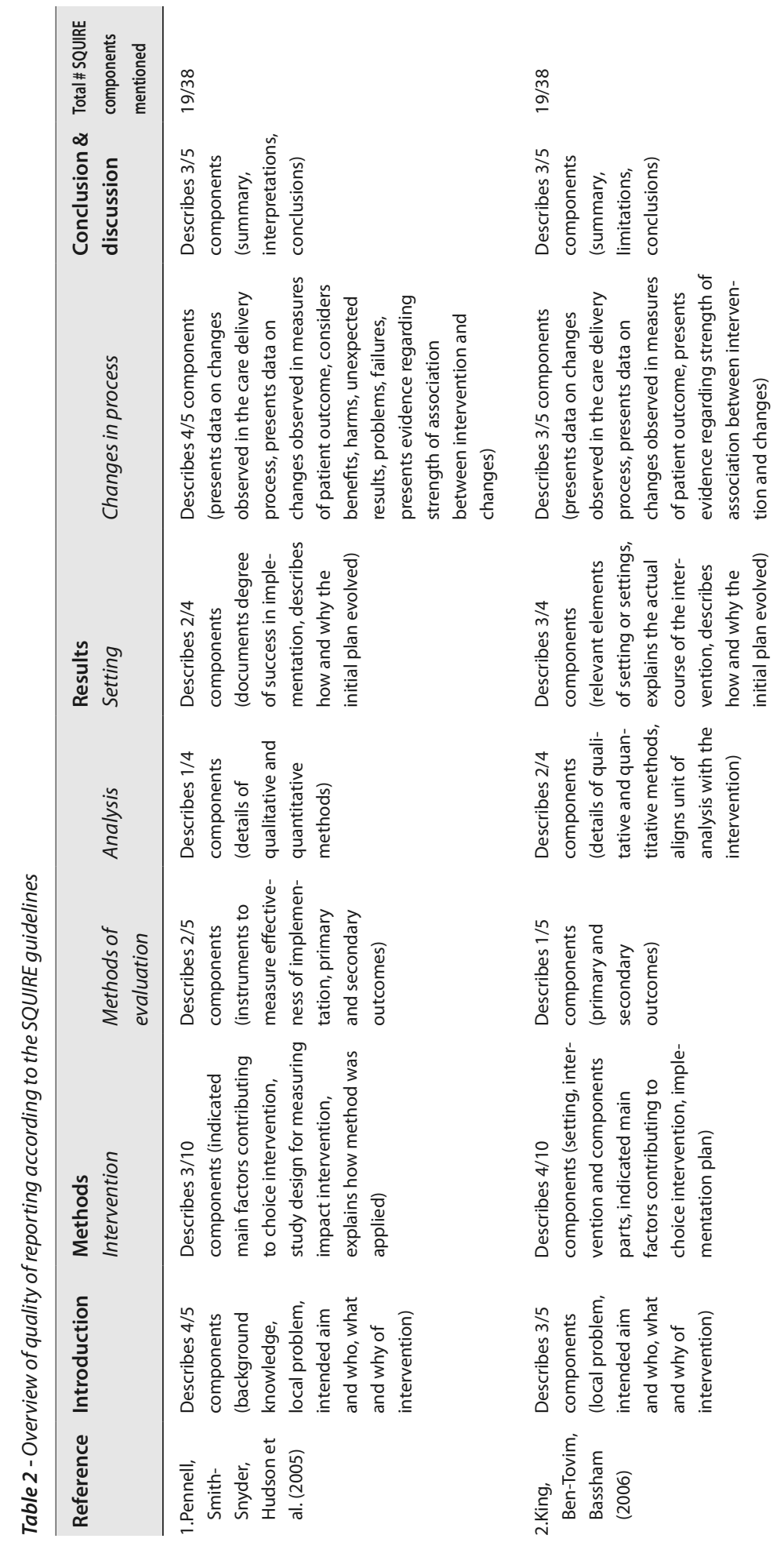














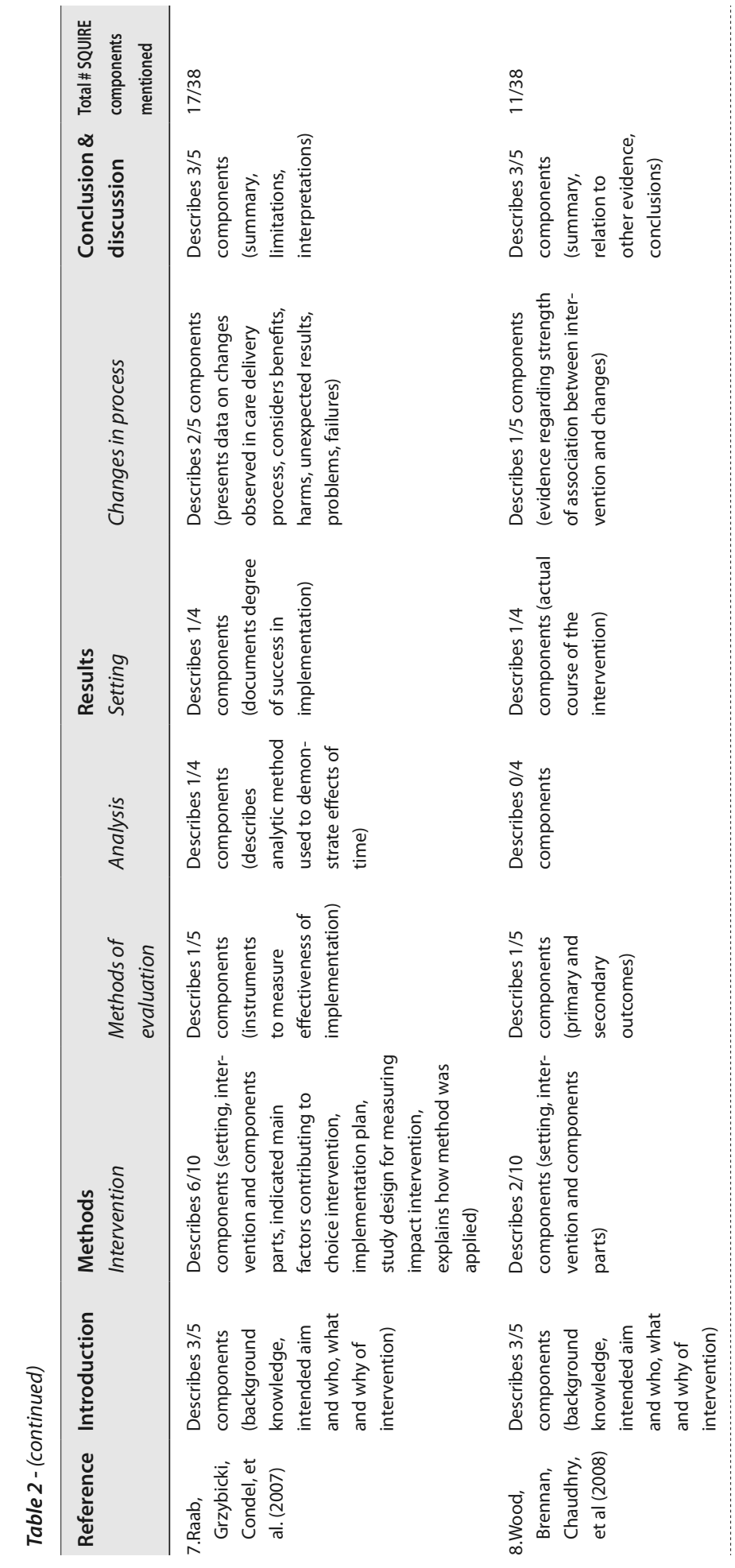




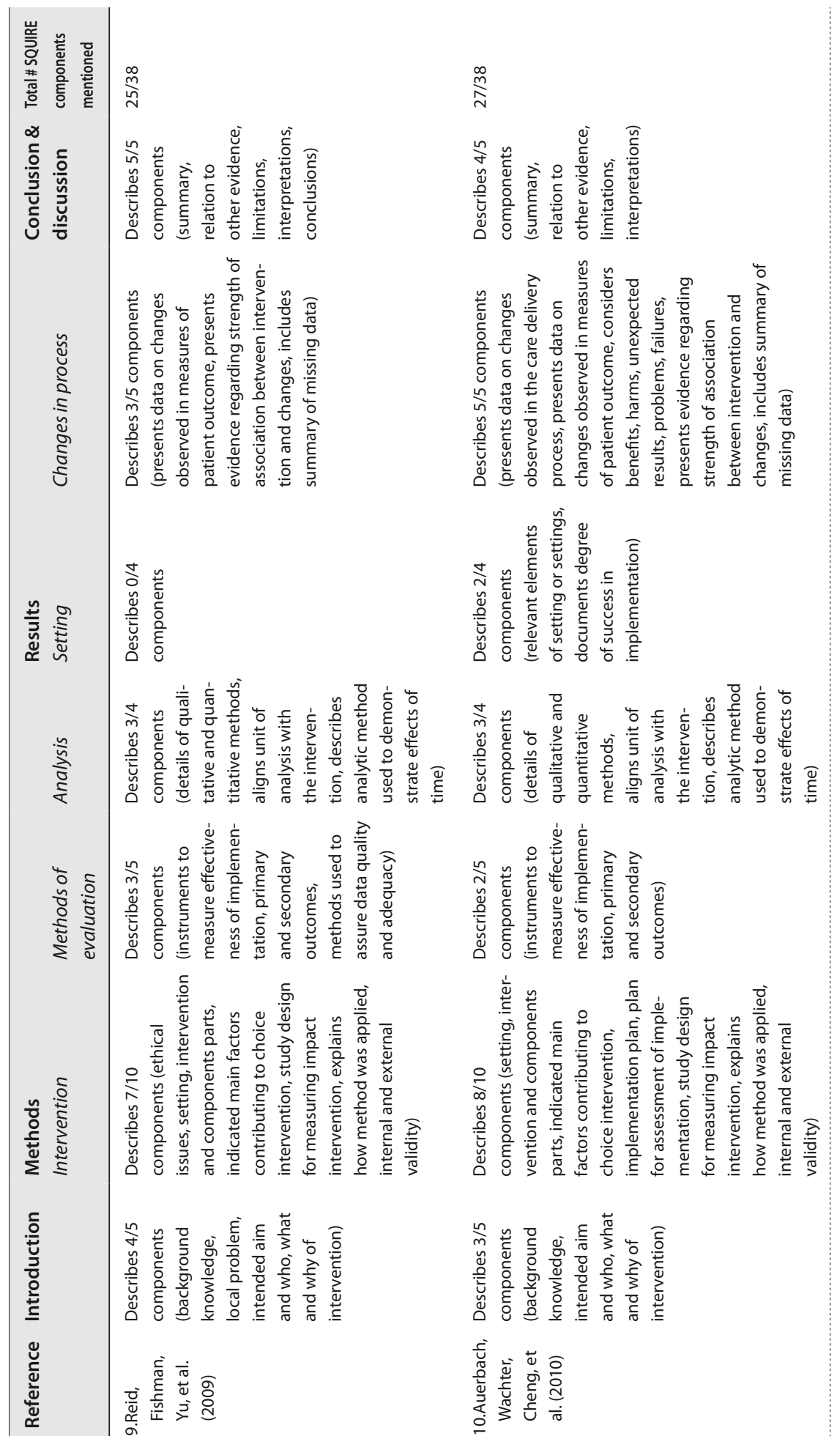




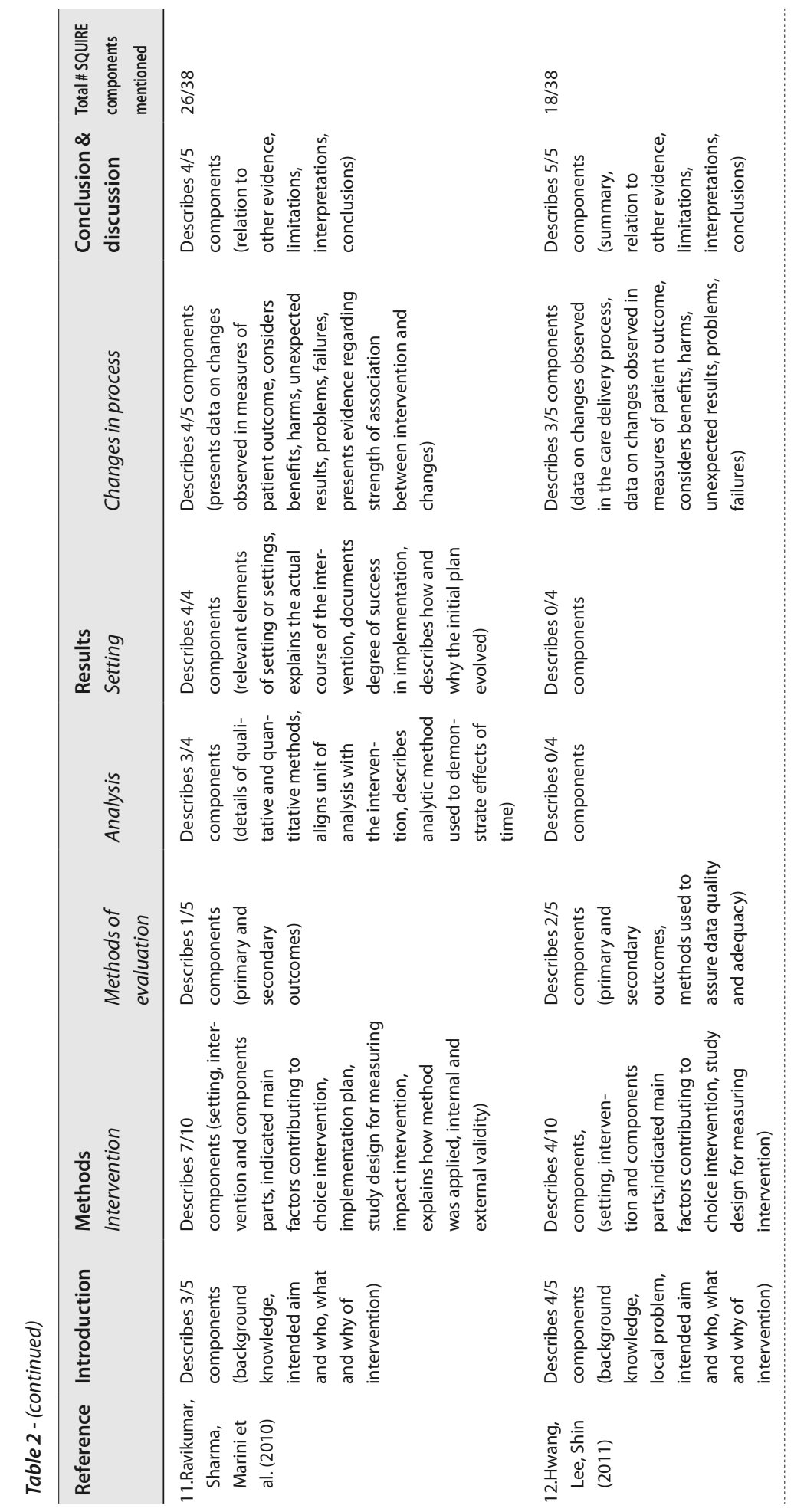




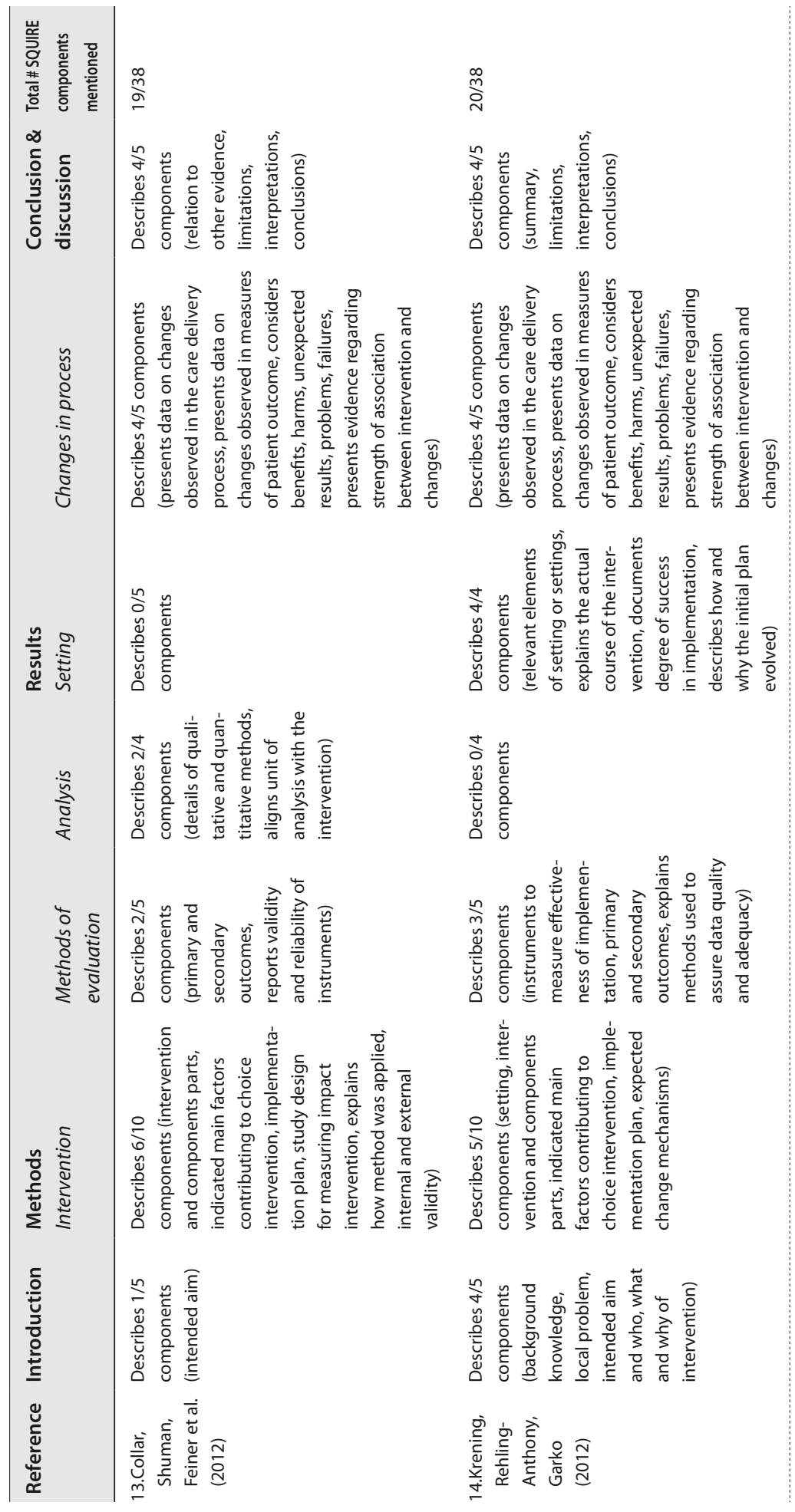




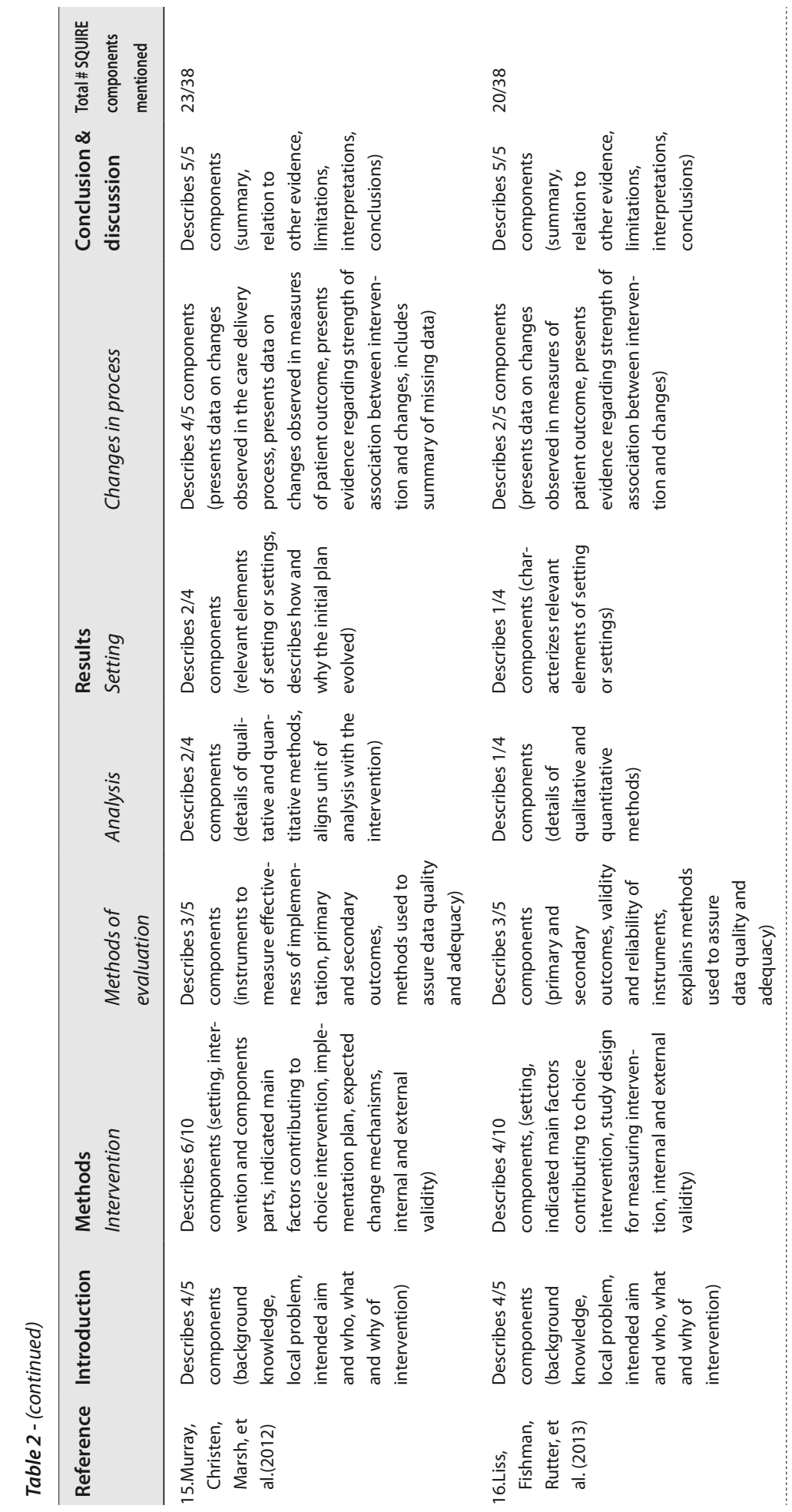




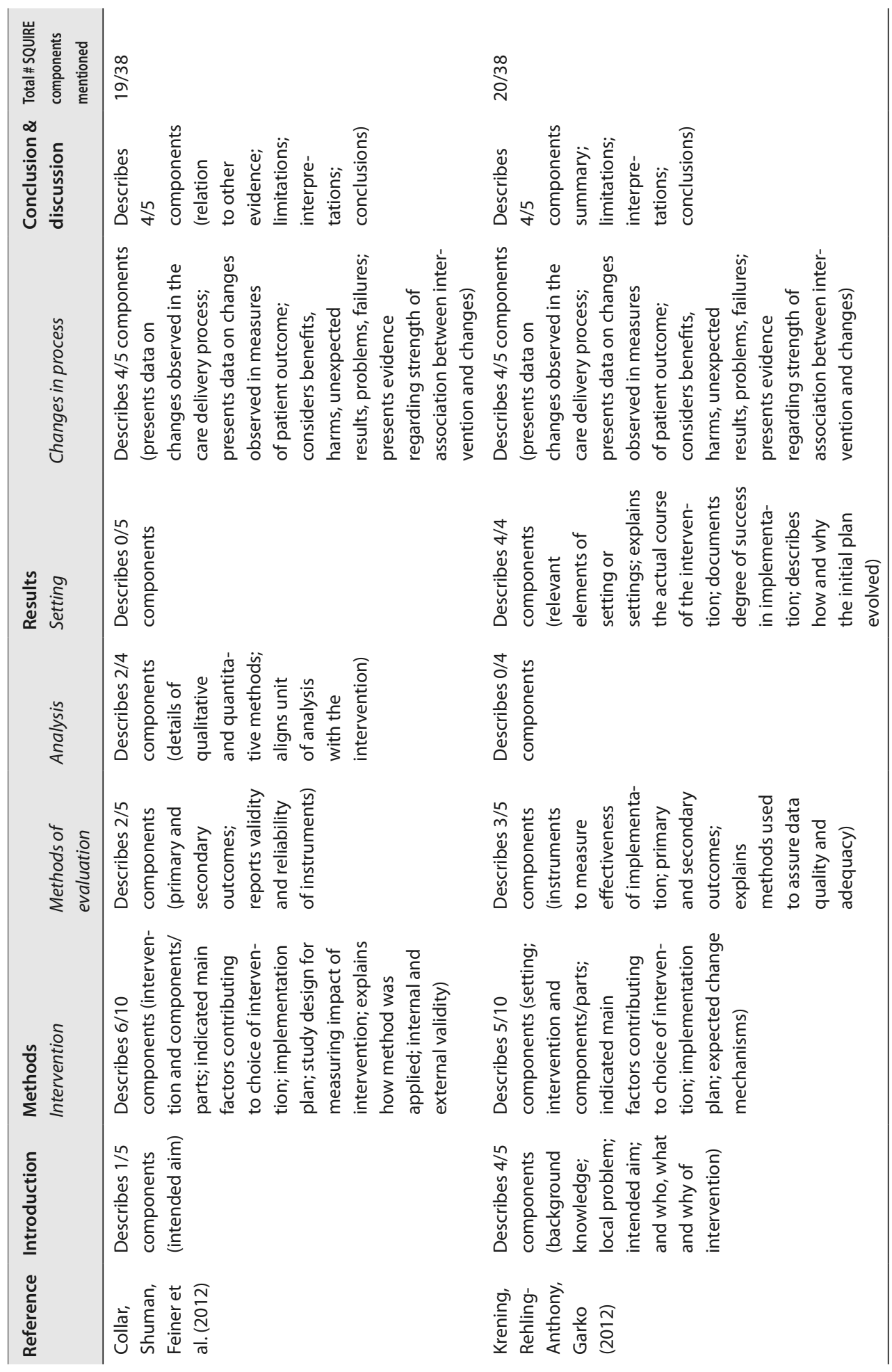




\section{Types of redesign interventions}

Table 3 summarizes the redesign interventions and study methods used. The objective of most studies was the implementation and evaluation of a specific redesign intervention. Improving quality of care was explicitly stated as an objective in seven studies ${ }^{12,15,18,20,23,25,26}$. Half of the redesign interventions implemented the approach known as lean thinking/Toyota production system $(\mathrm{N}=9)^{12,14,15,21,24-28}$. Two studies described the implementation of the concept of patientcentered medical home ${ }^{17,20}$, and three described more general forms of process redesign (structure redesign vs. process redesign ${ }^{23}$, evidence-based redesign ${ }^{18}$, nurse practitioner-led practice redesign $\left.{ }^{29}\right)$. Other interventions included a general process improvement project ${ }^{16}$, appreciative inquiry ${ }^{22}$, a hospitalist-led co-management neurosurgery service ${ }^{13}$ and a continuum of care $^{19}$.

Fourteen studies were performed in the USA ${ }^{12,13,15-17,19-22,25-29}$, two in Australia ${ }^{14,24}$, one in South $\mathrm{Korea}^{23}$ and one in Scotland ${ }^{18}$. Most took place in a hospital setting $(\mathrm{N}=12)^{13-16,19,21-24,27-29}$; others were conducted in primary care $(\mathrm{N}=3)^{12,17,20}$, a specialized clinic $(\mathrm{N}=1)^{18}$ or a laboratory $(\mathrm{N}=2)^{25,26}$. Length of follow-up ranged from three ${ }^{18}$ to $48^{27}$ months with a median of 12 months, though five studies did not mention its duration ${ }^{12,14,15,26,29}$. Patients were the most common unit of analysis $(\mathrm{N}=14)^{13-15,17-18,20-25,27-29}$. However, some studies reported on staff $(\mathrm{N}=2)^{12,21}$ or clinical notes $(\mathrm{N}=1)^{12}$ while a few did not define the unit of analysis $(\mathrm{N}=3)^{16,19,26}$. Mean sample size was $27,932.87(\mathrm{SD}=61,506.98)$, ranging from $49^{21}$ to $228,510^{20}$. Thirteen studies used a before-andafter design $(\mathrm{N}=12)^{12,14-16,20-24,27-29}$, while five used a controlled before-and-after design ${ }^{13,17,19,25,26}$. In summary, half of the redesign interventions were characterized as 'lean thinking' and took place in a hospital setting. Length of follow-up and sample size diverged widely, and most studies used an uncontrolled before-and-after design to evaluate the effectiveness of the intervention. 


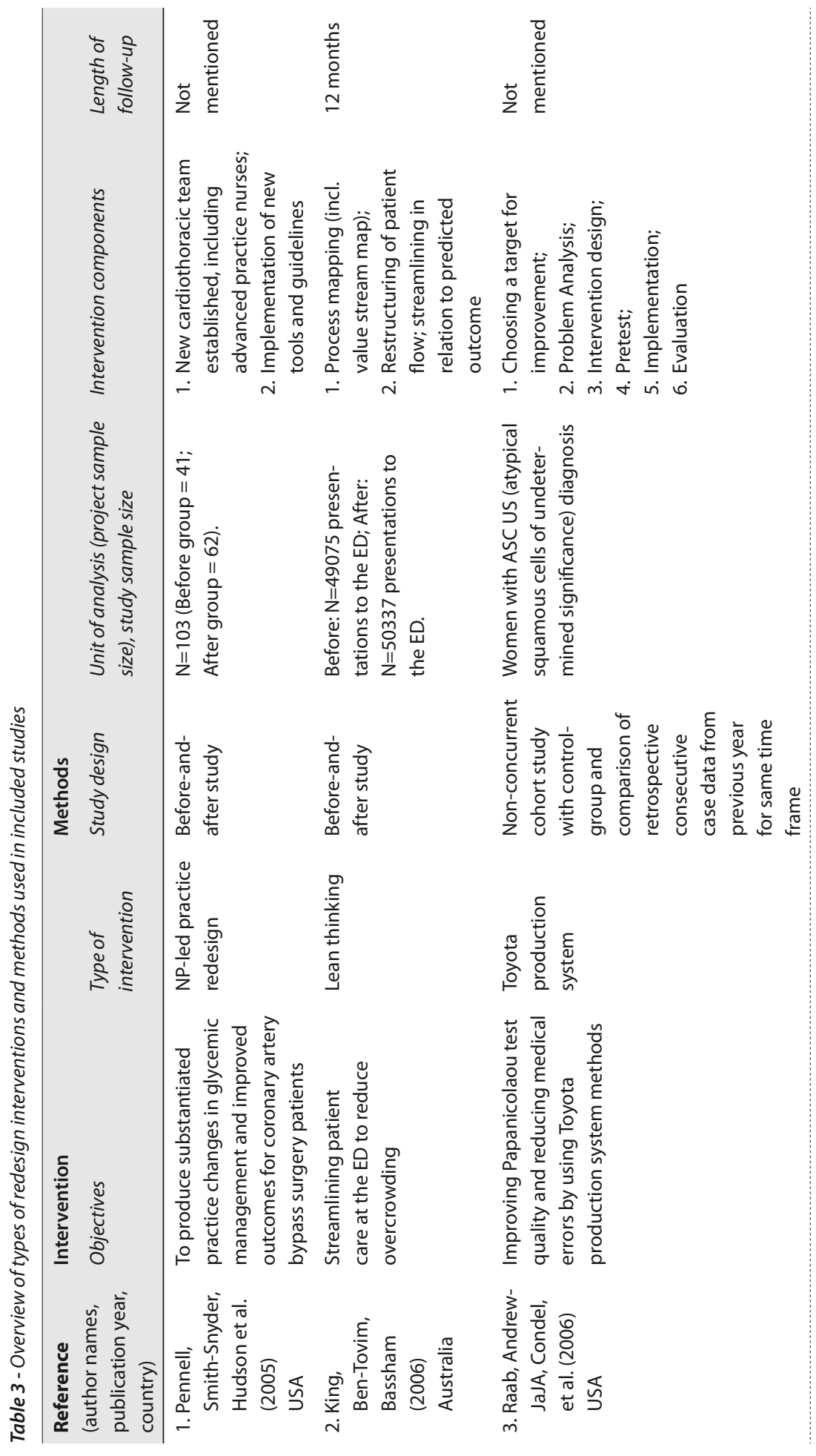




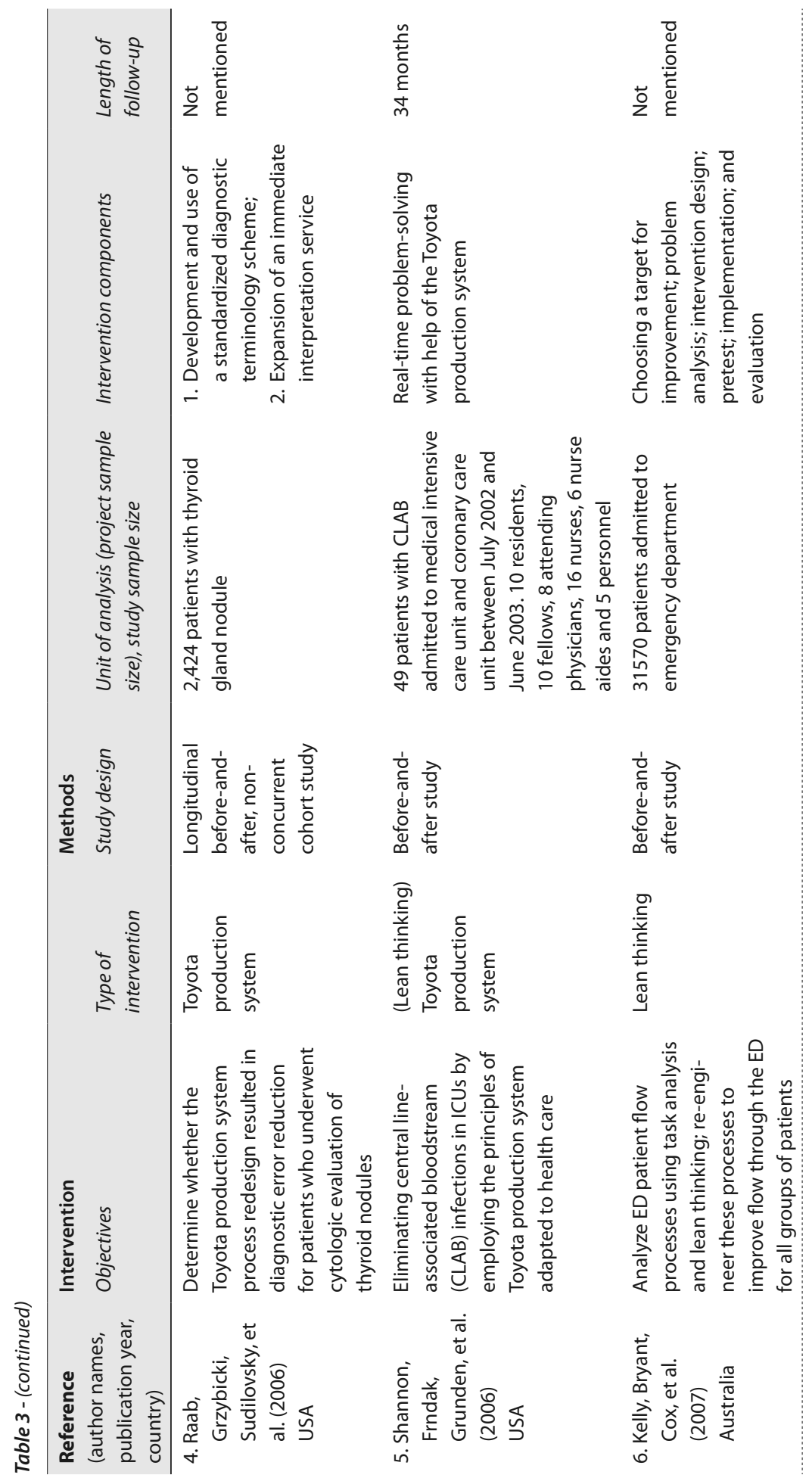




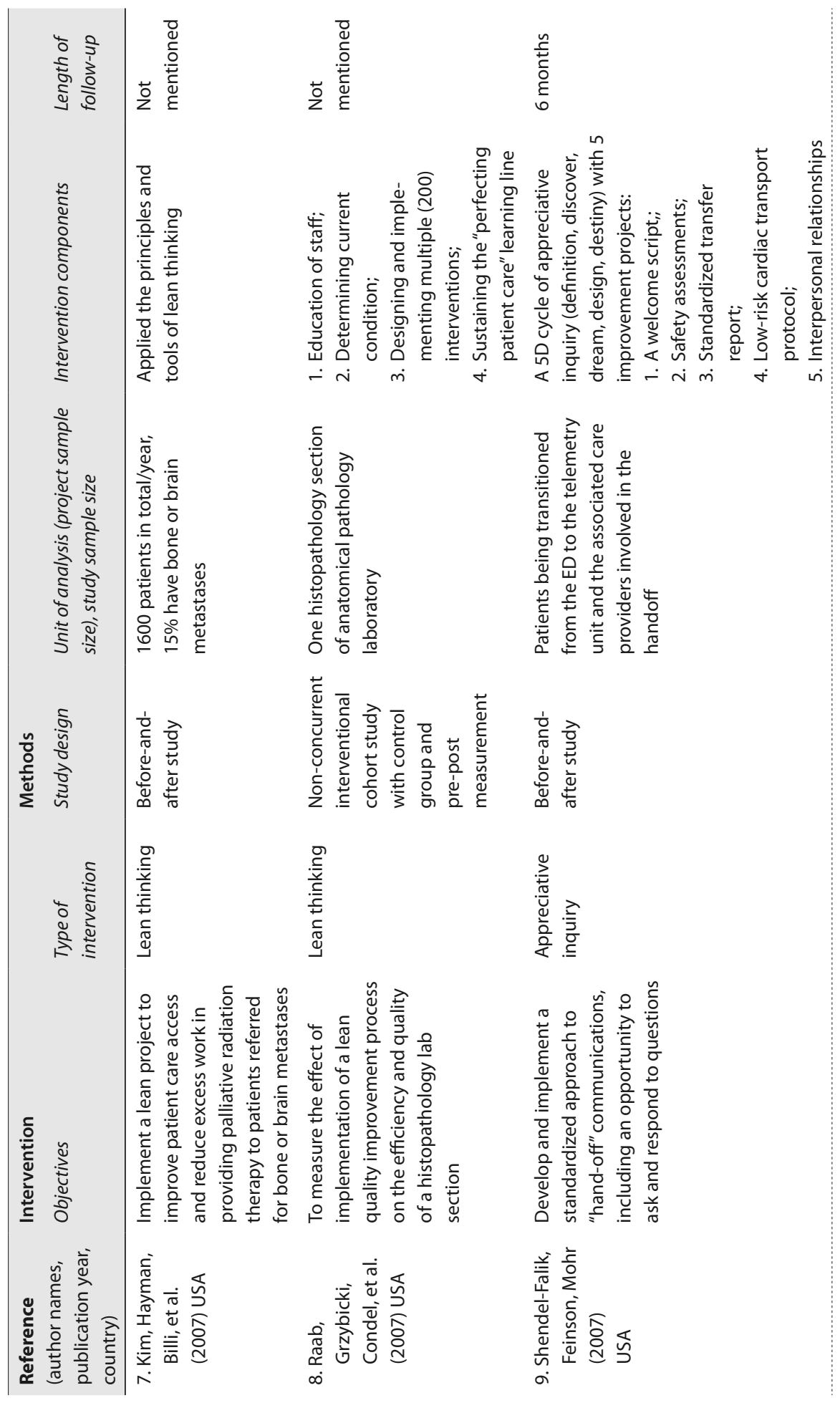









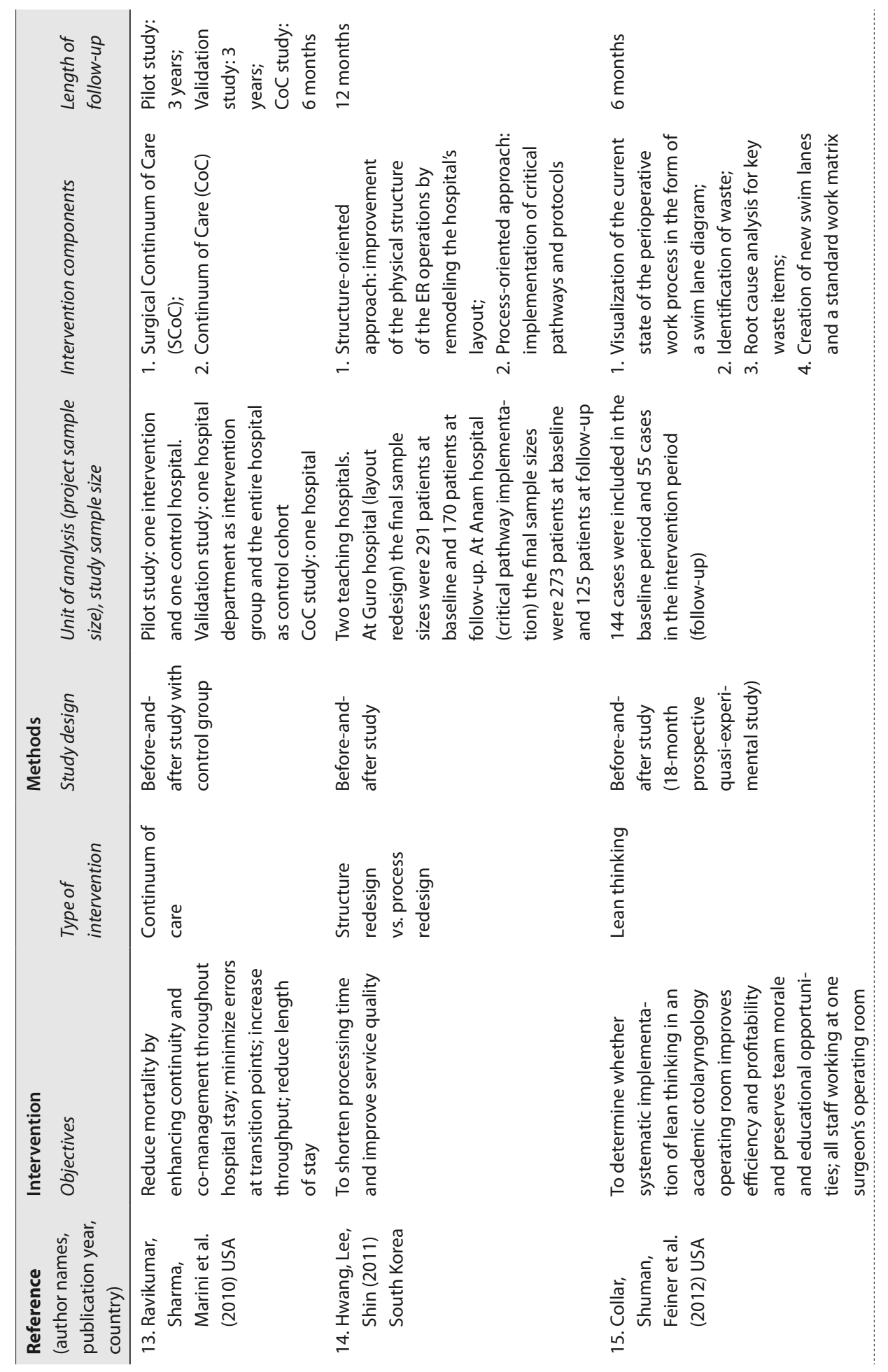




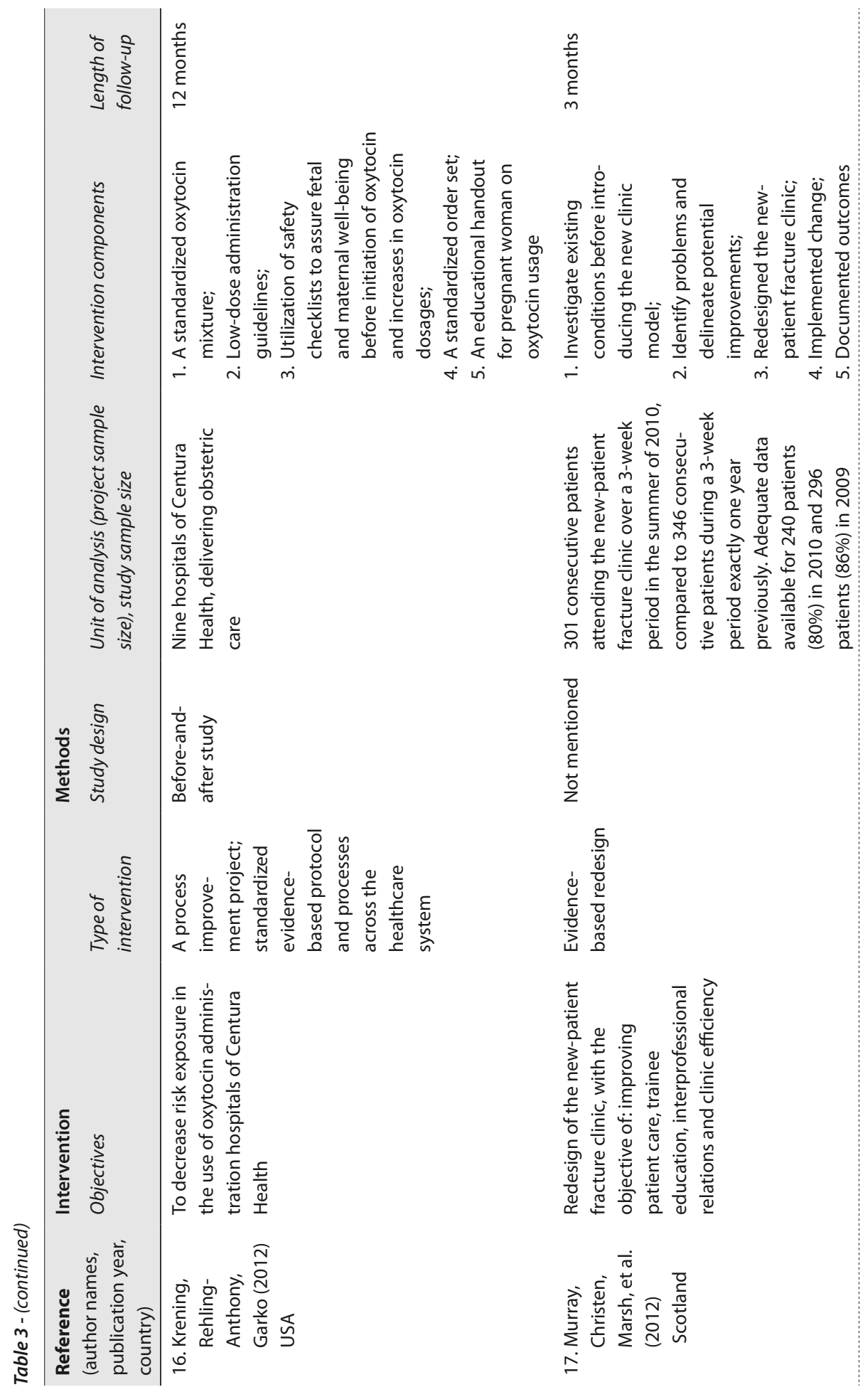




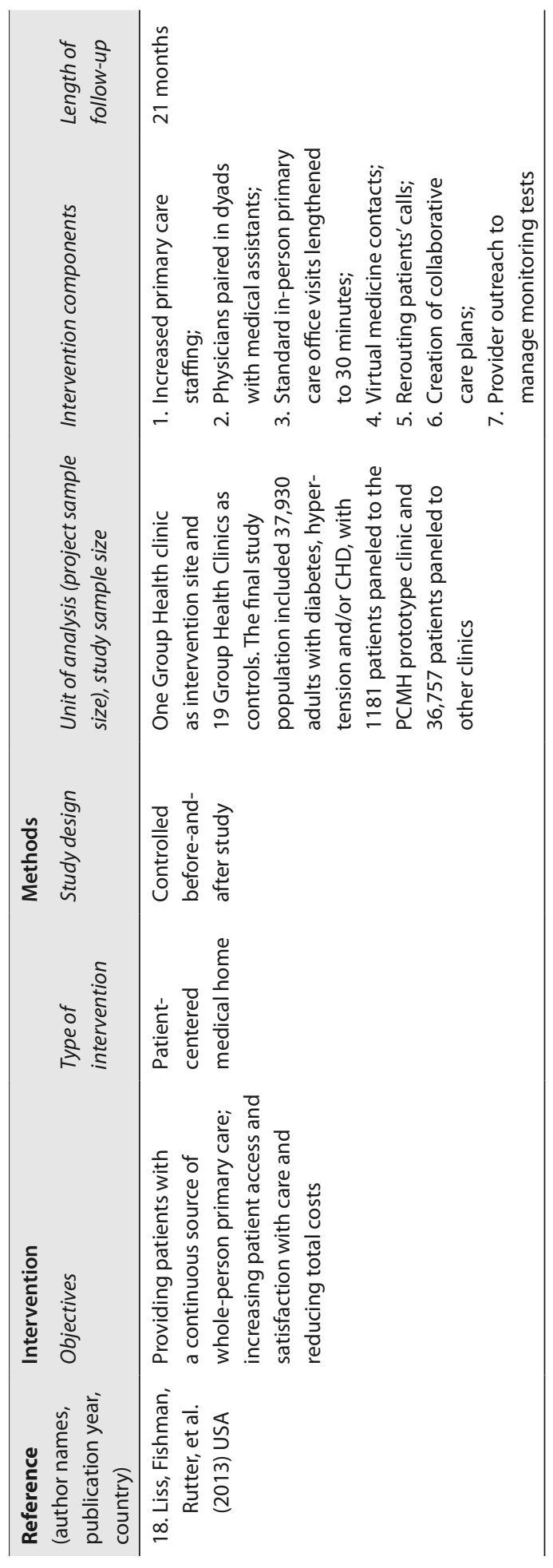




\section{Effects of redesign on quality of care}

Table 4 summarizes the outcomes of the studies. All reported improvements as a result of process redesign, while three ${ }^{14,20,23}$ also found declines in quality. Significant improvements were mentioned in 15 studies ${ }^{13,14,16-21,23-28}$, mostly gains in effectiveness ${ }^{16-21,25,27}$ and/or efficiency ${ }^{14,17-}$ $20,23,24,26,28$. Outcome measures showed great variance between studies. However, 'effectiveness' and'efficiency'were discussed most (1 1 studies reported on both dimensions ${ }^{13,14,16-22,25,29}$ ). Changes in efficiency were demonstrated by 17 studies $^{12-25,28,29}$. Efficiency was improved by decreasing

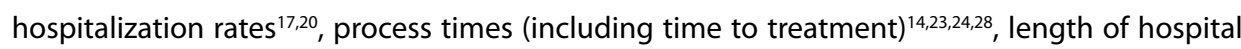
stay $^{19,23,29}$; by a shift in the writing of clinical notes ${ }^{12}$, savings on (estimated) $\operatorname{costs}^{13,16,19,20,25,28}$, raising provider productivity ${ }^{21,22,26}$ and reducing process steps and variability ${ }^{15,18,24,25}$. Efficiency also deteriorated: an increase was shown in process time for a sub-category of patients ${ }^{14,23}$, in specialty care visits ${ }^{20}$ and in specialty care costs ${ }^{20}$.

Changes in effectiveness were demonstrated in 12 studies $^{13,14,16-22,25,27,29}$. These reported improvements in disease conditions ${ }^{17,20,29}$ and adequate treatment usage ${ }^{16,22,29}$ as well as increases in discharged patients ${ }^{14,18}$ and diagnostic accuracy $y^{25,27}$.

Two studies ${ }^{14,15}$ found changes in timeliness as a result of process redesign, which reduced waiting time. Changes in patient-centeredness were demonstrated in three studies ${ }^{13,20,22}$ : improvements in patient satisfaction or experiences ${ }^{13,20,22}$; higher scores on doctor-patient interaction; and better coordination of care ${ }^{20}$. Changes in patient safety were found in 11 studies ${ }^{12,14}$ 16,18,19,21,24,25,27,29: increased physician identification ${ }^{12}$; improved documentation ${ }^{12}$; a decrease in complications ${ }^{14,16,19,21,29}$; fewer errors in routing patients to appointments ${ }^{15}$; fewer false-negative diagnoses ${ }^{25,27}$; and an overall sense of improvement in patient safety ${ }^{24}$.

None of the studies measured equity of care. Eight mentioned other outcomes unrelated to the six quality dimensions, such as changes in provider satisfaction ${ }^{12,22}$, staff perceptions of the implemented change $\mathrm{e}^{13,14,18,21}$, changes in team morale ${ }^{28}$, or changes in incident rates ${ }^{18}$. 


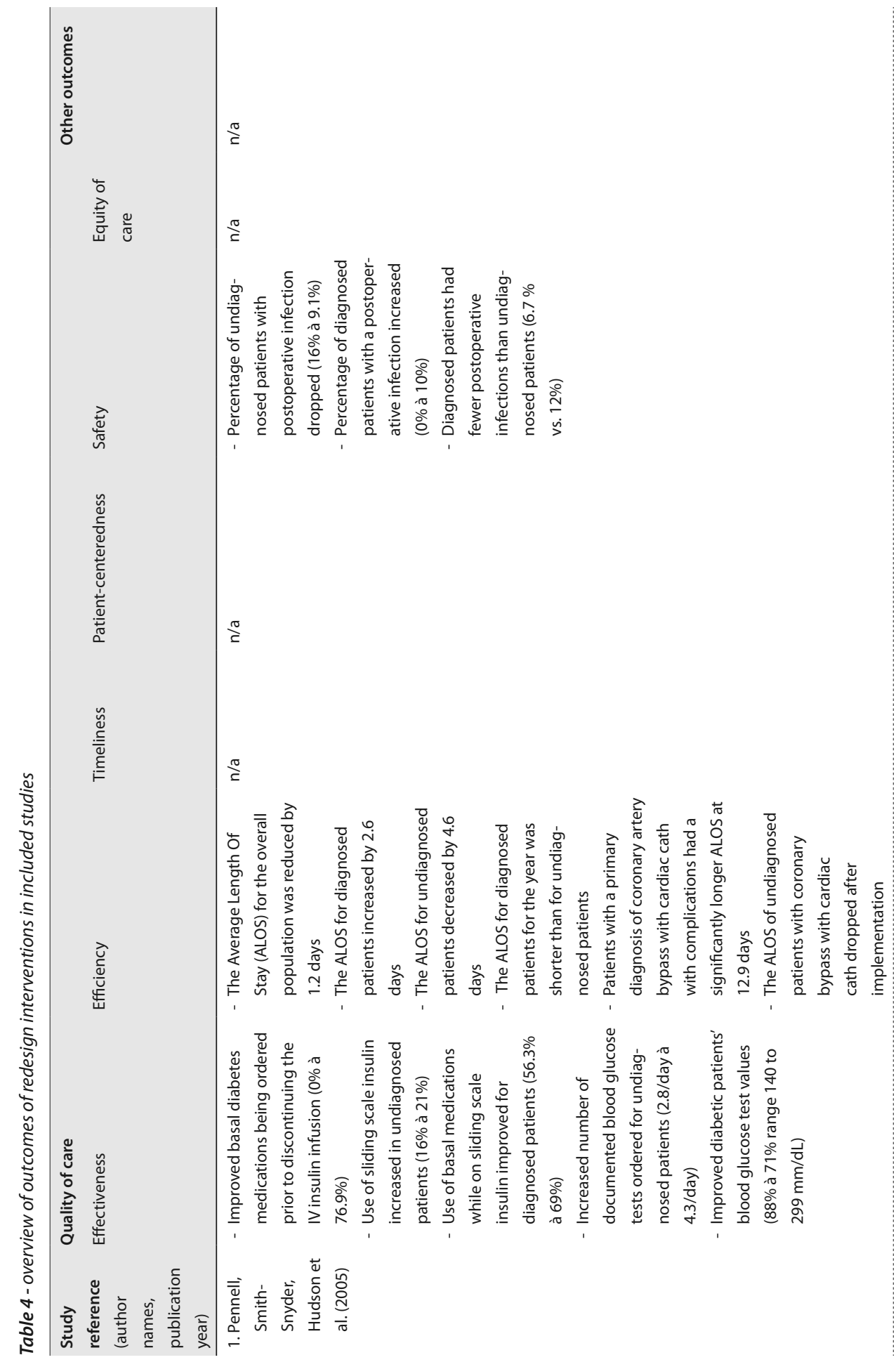




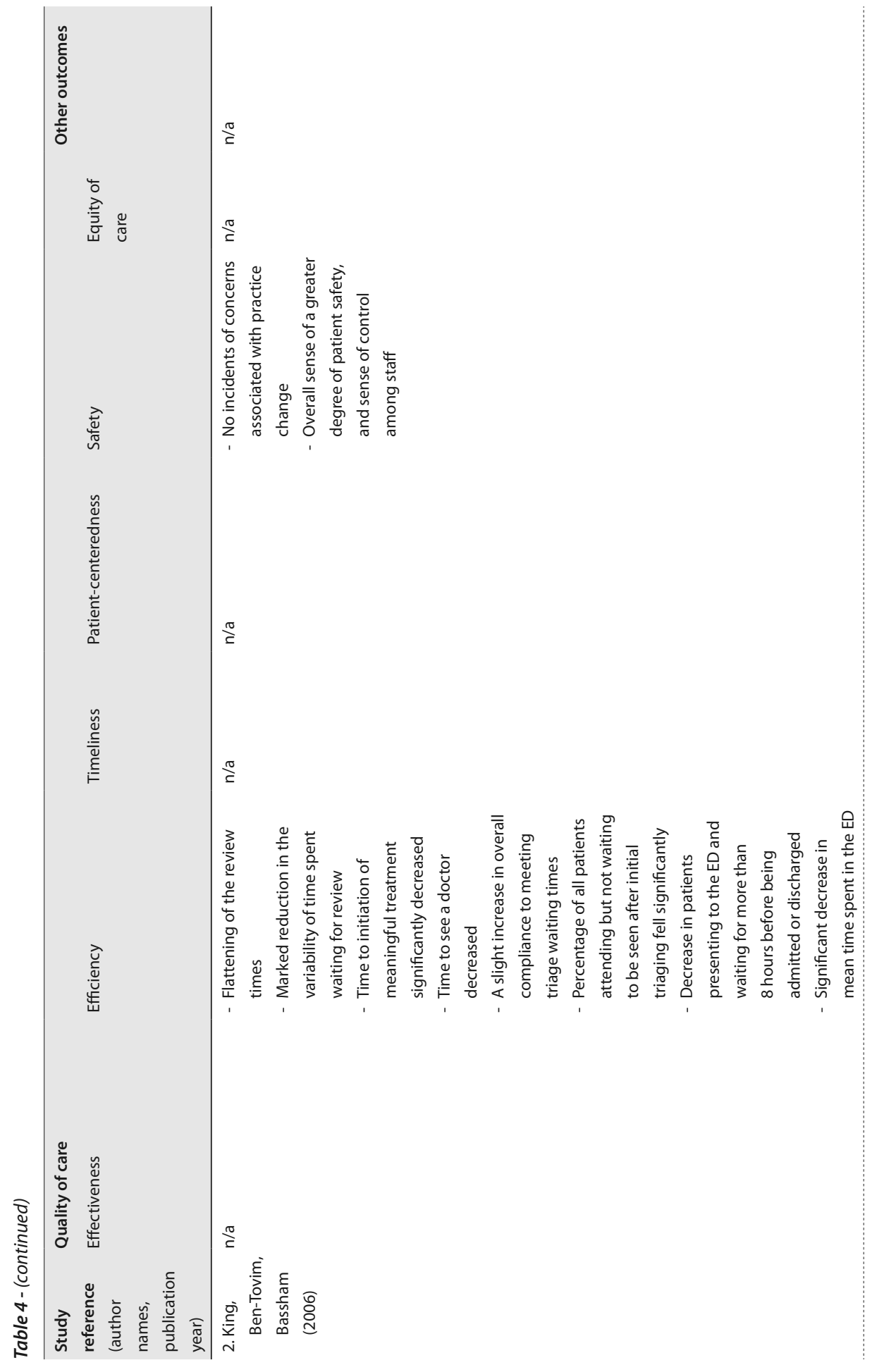


The impact of redesigning healthcare processes

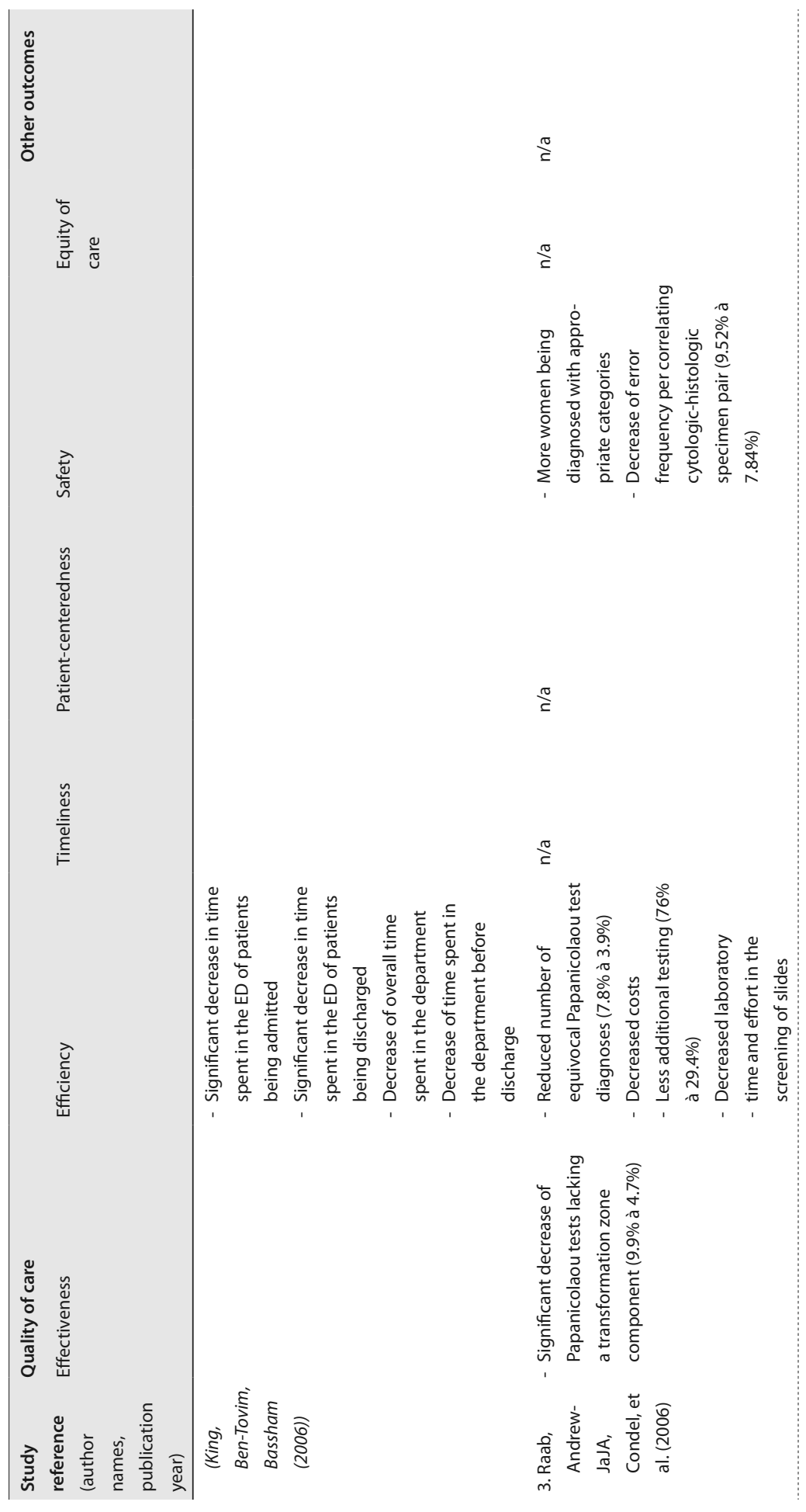




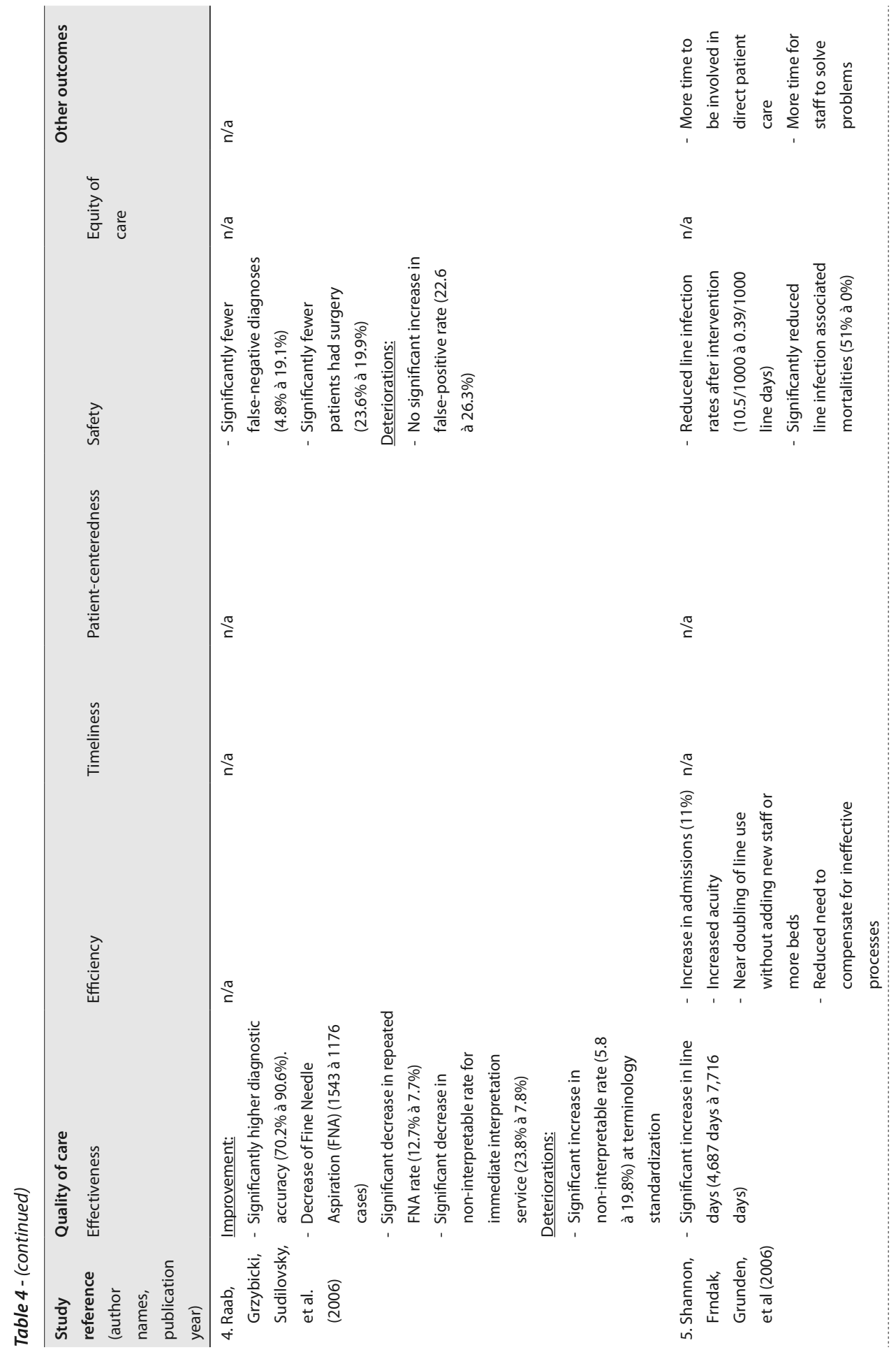




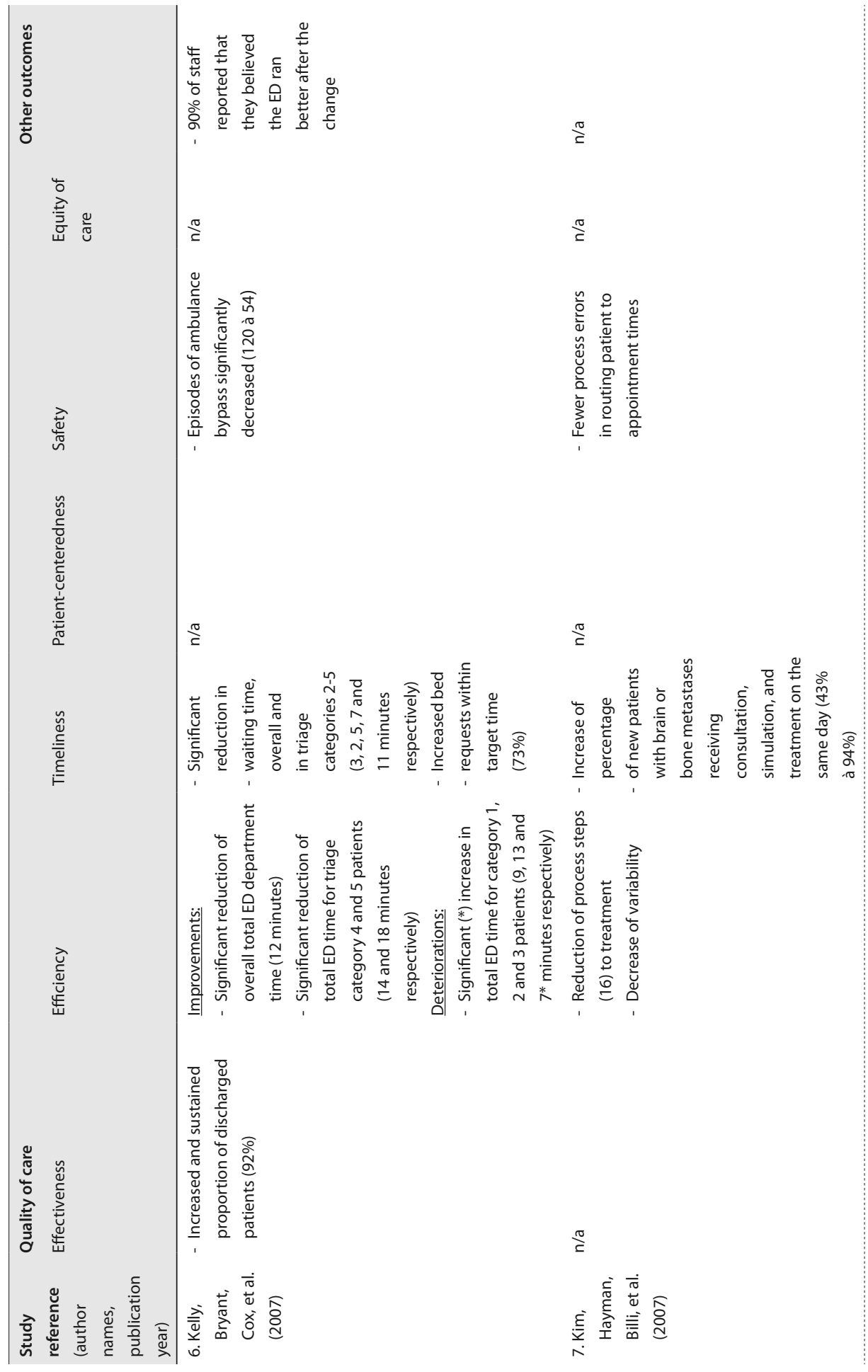




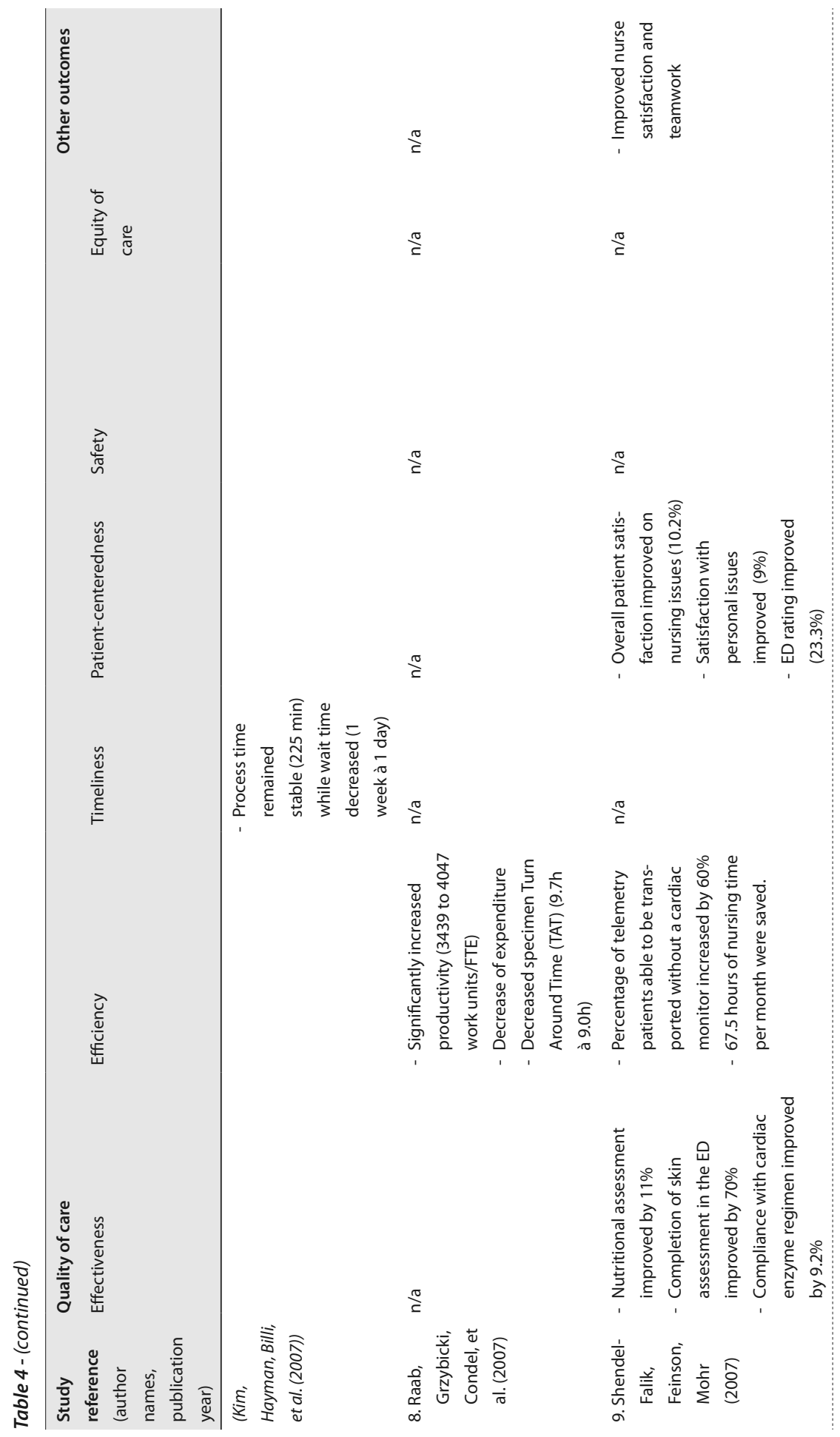




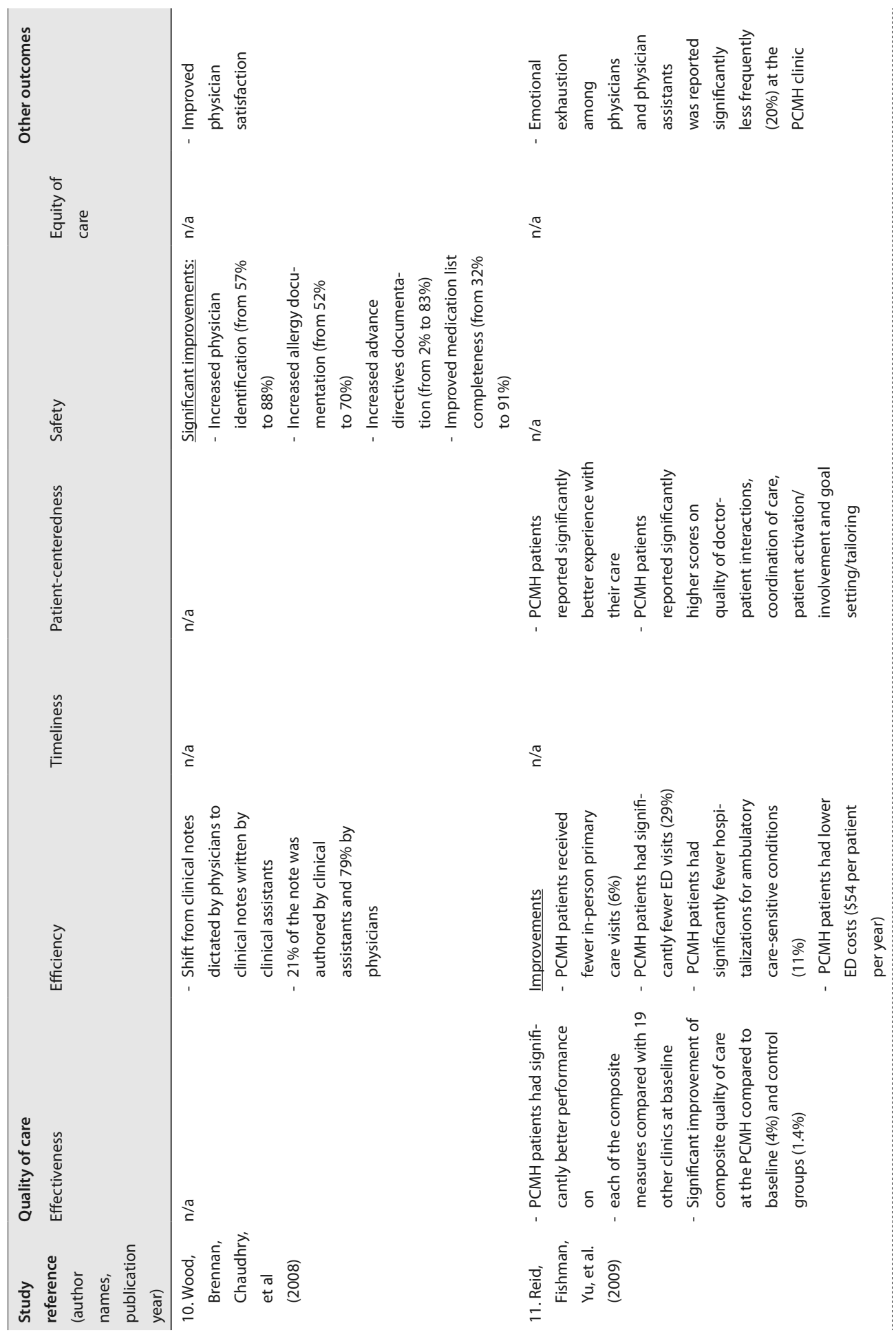




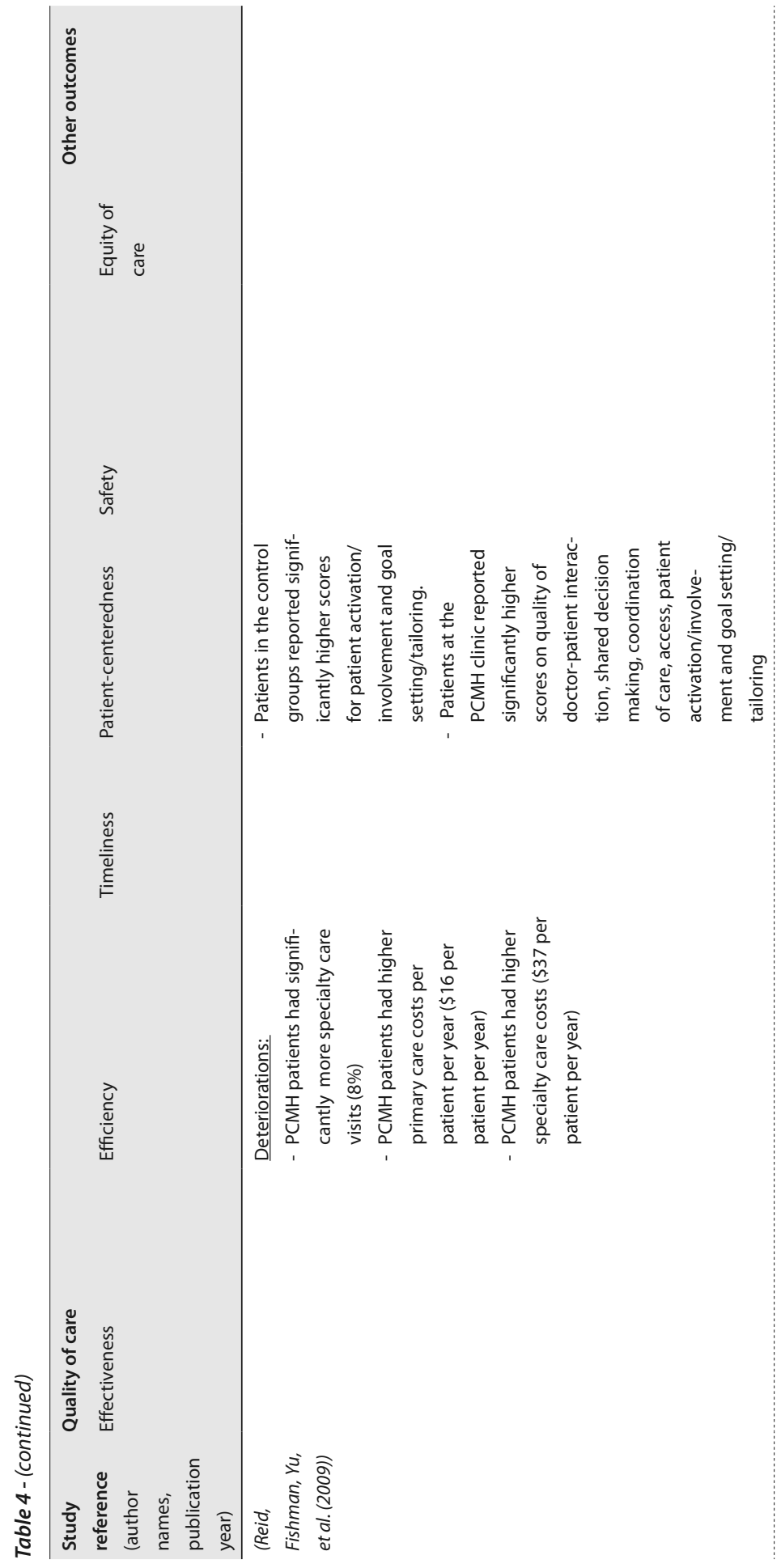




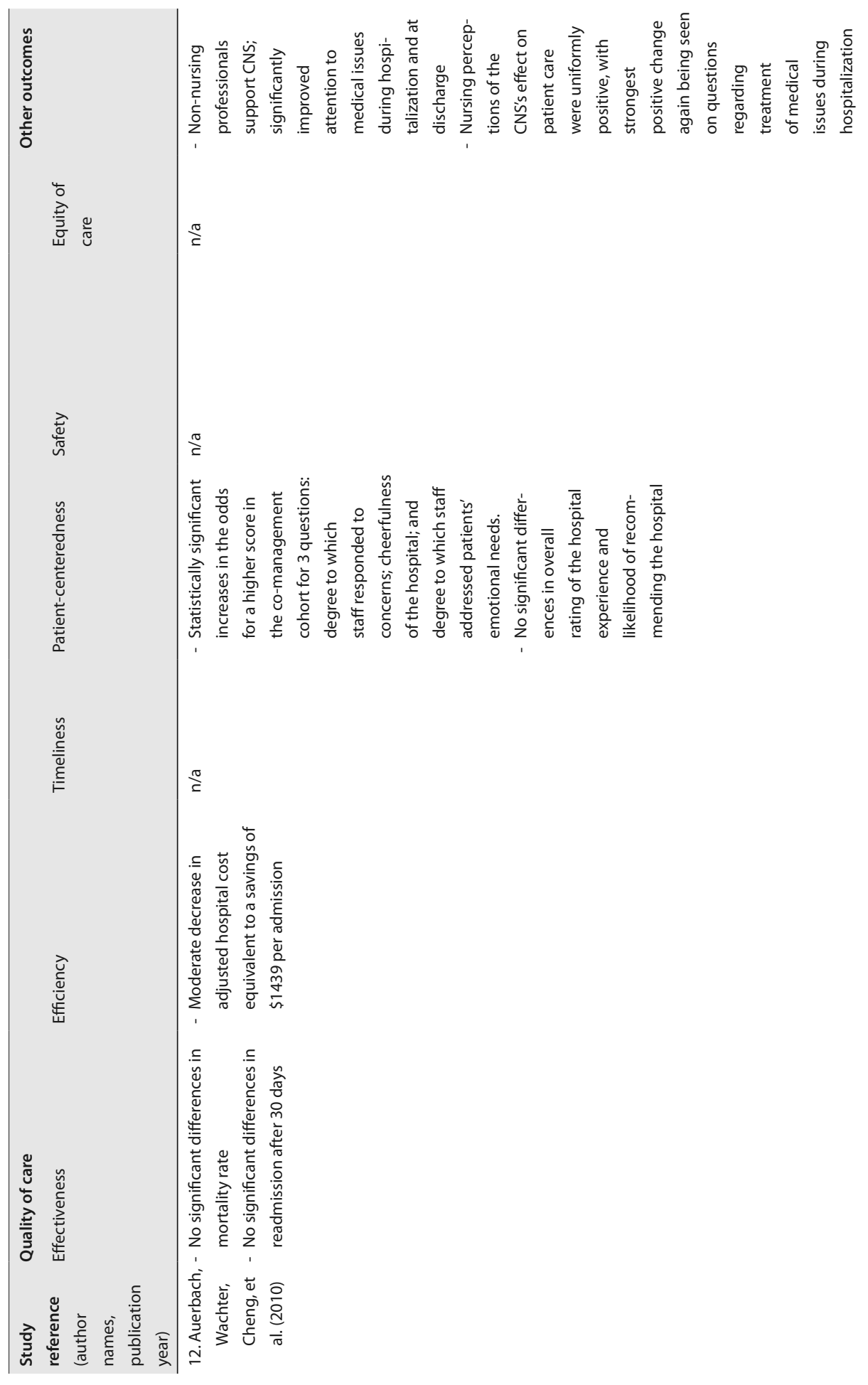









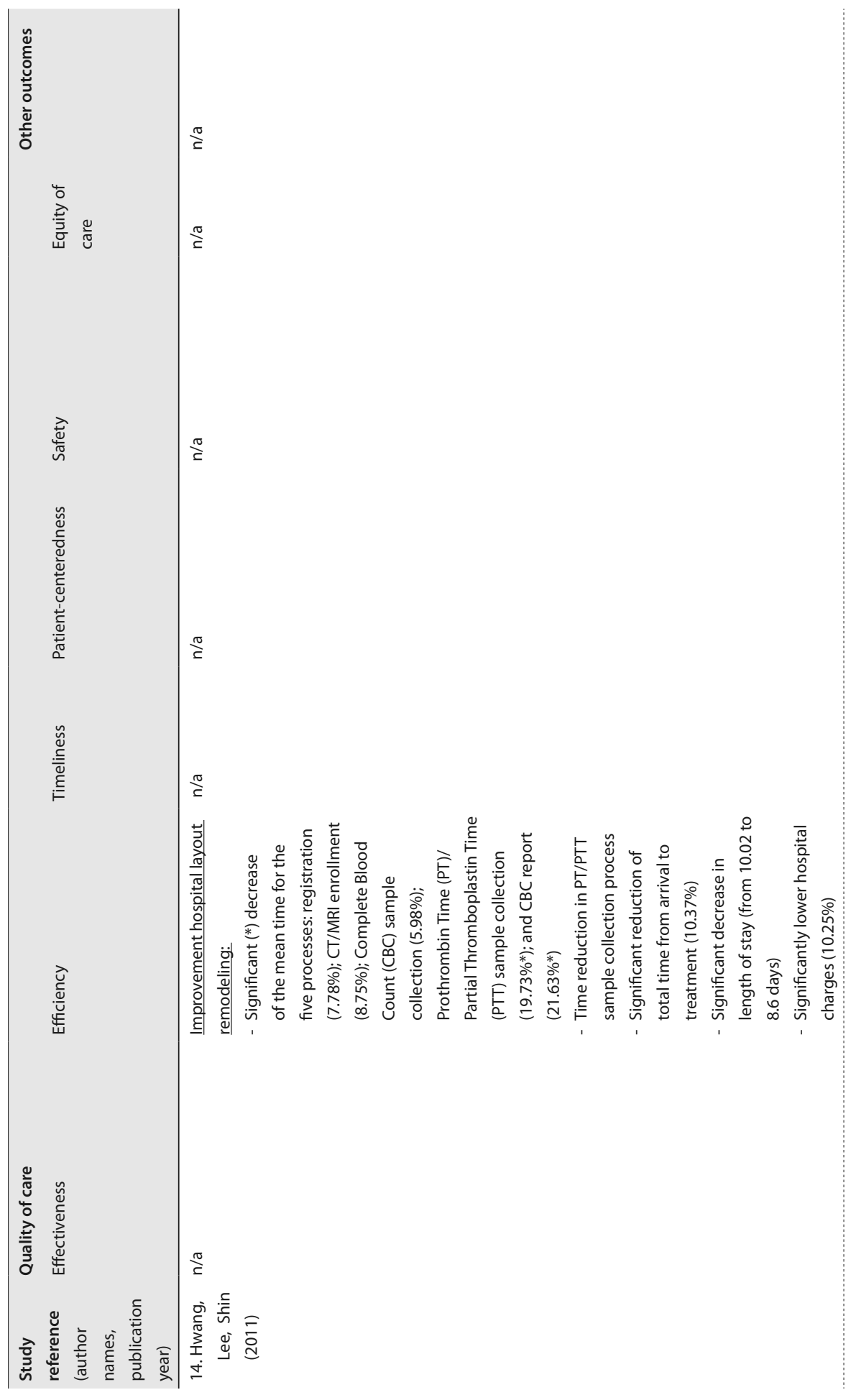









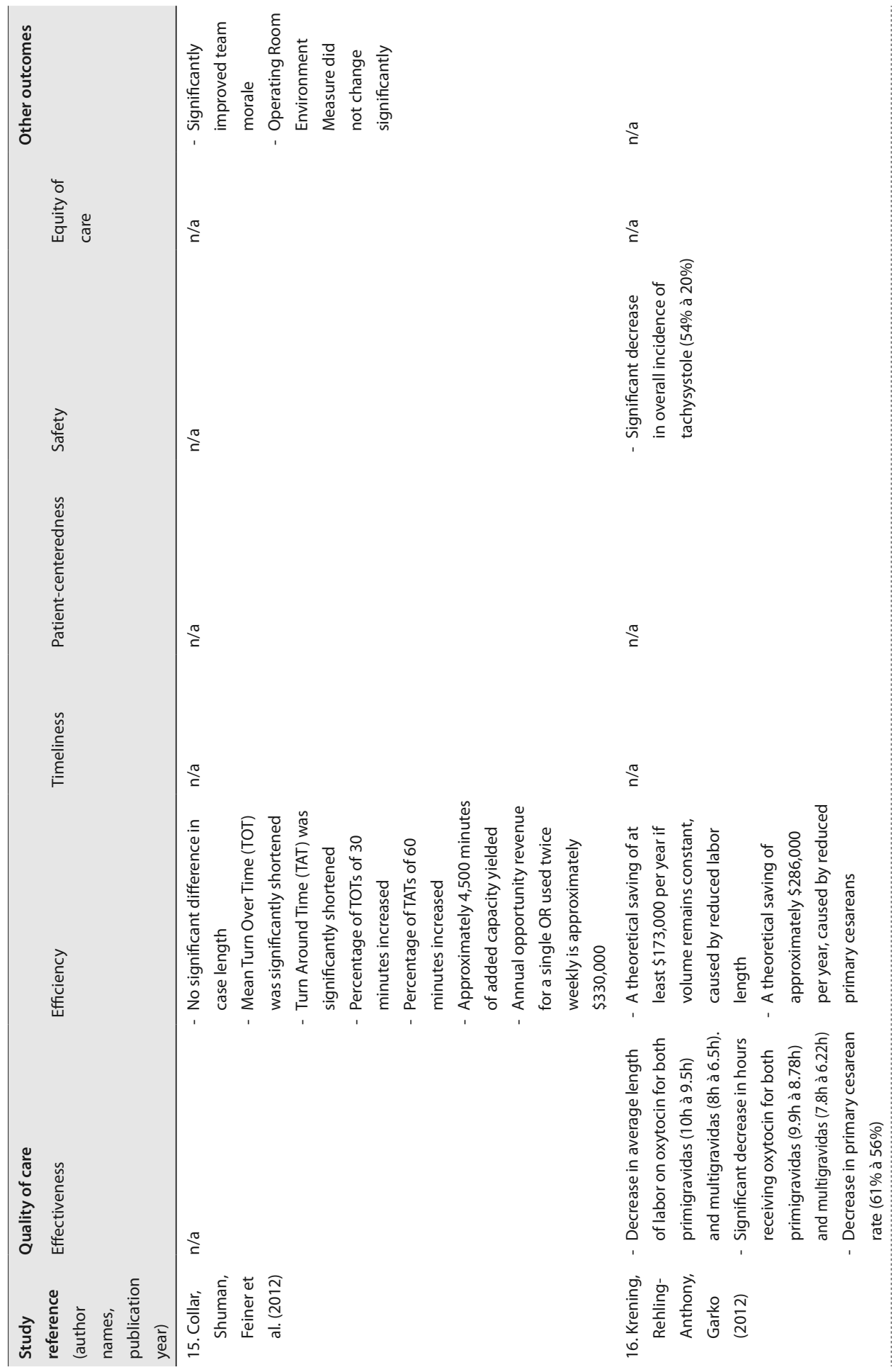









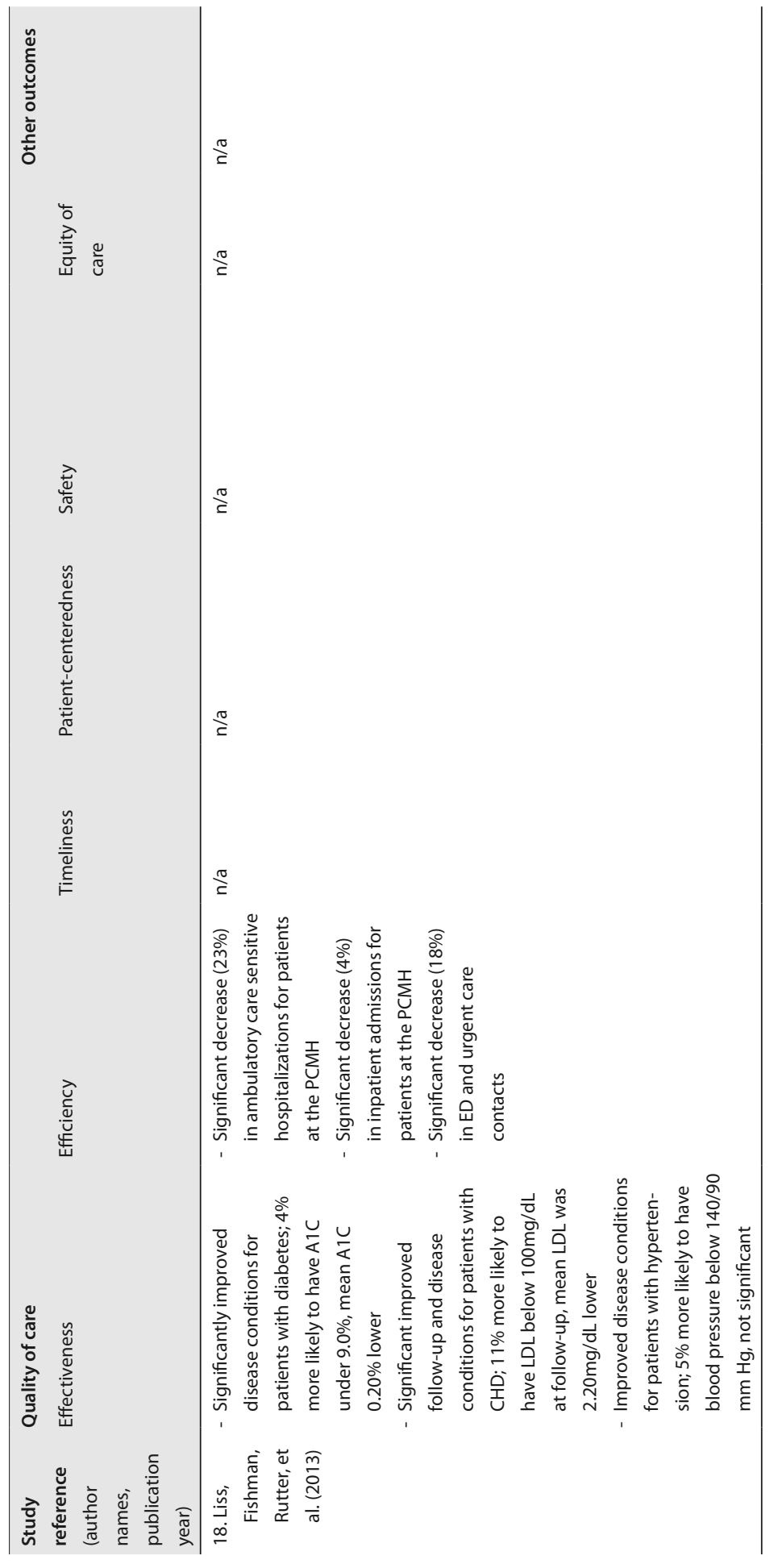




\section{DISCUSSION}

The need to redesign healthcare processes in order to address deficits in quality of care and create more sustainable care processes is acknowledged worldwide ${ }^{2,3,5}$. The effects of process redesign have not been clearly described, however ${ }^{5,6}$. By synthesizing evidence from 18 studies in the international literature, this systematic review contributes to a better understanding of the influence of process redesign interventions on quality of care. It suggests that they have positive effects on certain aspects of quality. However, the full impact cannot be determined on the basis of the literature. Studies differed in the type of redesign implemented, study setting, methods used for evaluation, and outcome measures. All types of intervention seemed to improve outcomes in one or more respects. Nonetheless, it is not clear which type of redesign has the most potential in a particular setting. Efficiency, effectiveness and patient safety gains were best described in the included studies, while the effects on patient-centeredness, timeliness and equity of care received little attention.

Applying the SQUIRE guidelines demonstrated that overall the reporting was weak. Given the study designs, the results are subject to bias, as changes in the research settings might be responsible for the effects ${ }^{30,31}$. In addition, changes in process might have been induced by background factors ${ }^{31}$. Longitudinal effects of redesign interventions were hardly evaluated, as follow-up varied from three to 48 months with a median of 12 months. The methodological problems of studies reporting on quality improvement interventions like process redesign are well known ${ }^{6,31-34}$. Yet the methodology of the studies covered here was no better than in preceding studies. These weaknesses form potential threats to the internal and external validity of the findings. Unless a more uniform and robust evaluation of process redesign interventions is carried out, general conclusions cannot be drawn about their impact on quality of care.

To the best of our knowledge, this is the first systematic review of the effect of process redesign on quality of care, using broad definitions for both study setting and types of redesign. Elkhuizen et al. ${ }^{6}$ performed a systematic review of the evidence of business process redesign in hospital settings until 2004. However, that review included studies combining multiple interventions, which made comparison impossible. Those authors concluded that studies were hard to find and lacked a clear and consistent research methodology. In that light, they recommended the development of reporting guidelines.

Specific redesign interventions have been reviewed recently. In one, Mazzocato et al..$^{35}$ reviewed the 'lean-thinking' literature from a realist perspective, focusing on the mechanisms through which 'lean thinking' operated. The authors identified positive effects of lean implementation in all included studies and common contextual factors interacting with components of the lean interventions that triggered the change mechanisms. Here too, the use of unclear study designs or outcome measures is mentioned. The authors suspect publication bias, as only positive effects were being reported. 
The impact of quality-improvement collaboratives was reviewed by Schouten et al. ${ }^{36}$. Although the outcomes were positive, the strength of evidence was limited by methodological constraints due to weak study designs, and the authors suspect positively biased findings. Implementation of the concept 'patient-centered medical home' was reviewed by Jackson et al. ${ }^{37}$, who showed small positive effects on patient experience and care delivery. There too, the strength of evidence was moderate to low. Publications were hard to find, evidence was fragmented, and comparison between studies was hard if not impossible.

The findings of the present review are therefore in line with those of earlier studies on this topic in the sense that a broad perspective on redesign interventions and settings generates similar results.

\section{Limitations}

Even though a systematic approach guided this review, the findings might be subject to some bias, which should be kept in mind when interpreting them.

First, publication bias might be present: most of the studies report on positive findings, and there is a general tendency in scientific literature to over-represent positive results ${ }^{38}$. As previous research on this topic also raised concerns about publication bias, this issue is pertinent to this review too. It is unlikely that using predefined redesign concepts would have addressed this problem, as publication bias was a concern in reviews that did use such concepts[35], underlining the need to report all outcomes of redesign in healthcare.

Second, limiting the scope by only including studies that used before-and-after measurement might have led to some selection bias. Nonetheless, limiting the search strategy did ensure a solid basis for comparison of the effects of the redesign interventions.

Third, since the terminology used to describe the interventions varies greatly, we could have missed some relevant studies. We circumvented this problem by searching multiple databases with database-specific headings like $\mathrm{MeSH}$ terms and amplifying the strategy by searching with free-text words.

Fourth, the SQUIRE guidelines might not be the only instrument for assessing excellence in reporting. Although they were specifically developed to assess reporting excellence for this type of studies, the checklist does not provide a value judgment on the methodology (or strength of evidence) of the studies ${ }^{11}$. Nonetheless, by covering methodological components, the SQUIRE checklist gives a sense of the methodological strengths of a study.

Finally, using the loM dimensions of quality of care might have made it difficult to compare findings across studies. Since the loM does not specify which outcome measures belong to the six dimensions, there is room for interpretation. Even though this might have influenced the presentation of findings in this review, using the loM dimensions facilitated classification of the outcomes, thereby revealing gaps in the research literature. 


\section{CONCLUSION}

Scientific evidence supporting process redesign in healthcare is limited and inconsistent. Outcome measures for the effect of redesign interventions vary across studies to the extent that it is impossible to draw conclusions about the impact on overall quality of care, or even on some of its dimensions. The findings of this systematic review suggest that the evaluation of process redesign interventions should be improved to reveal their full effect. It should meet the basic standards for reporting (SQUIRE guidelines) and apply more robust research designs. The influence of process redesign on patient-centered care, equity of care and timeliness warrants further research, applying outcome measures that capture the full scope of quality of care. Current research tends to ignore the long-term effects of process redesigns. Robust evaluations of their implementation should also identify the mechanisms through which effects were realized. This would help researchers and policymakers determine the value of specific interventions and offer an overview of improvement efforts that is less fragmented. 


\section{REFERENCES}

1. Hofmarcher MM, Oxley H, Rusticelli E. Improved Health System Performance Through Better Care Coordination. OECD Health Working Paper No 30. Paris: The Organisation for Economic Co-operation and Development.;2007.

2. Institute of Medicine. Crossing the Quality Chasm: A New Health System for the 21st Century. Washington, DC: National Academies Press; 2001.

3. OECD. Health at a Glance: Europe 20122012.

4. Fuchs VR, Emanuel EJ. Health care reform: Why? What? When? Health Aff. 2005;24(6):1399-1414.

5. Institute of Medicine. Performance Measurement: Accelerating Improvement. Washington, D.C.: Institute of Medicine;2006.

6. Elkhuizen SG, Limburg M, Bakker PJ, Klazinga NS. Evidence-based re-engineering: re-engineering the evidence--a systematic review of the literature on business process redesign (BPR) in hospital care. Int J Health Care Qual Assur Inc Leadersh Health Serv. 2006;19(6-7):477-499.

7. Vest JR, Gamm LD. A critical review of the research literature on Six Sigma, Lean and StuderGroup's Hardwiring Excellence in the United States: the need to demonstrate and communicate the effectiveness of transformation strategies in healthcare. Implement Sci. 2009;4:35.

8. Harmon P. Business Process Change. A manager's guide to improving, redesigning and automating processes. San Francisco, CA: Morgan Kaufmann Publishers; 2003.

9. Donabedian A. An introduction to Quality Assurance in Health Care. Orford: University Press; 2003.

10. Moher D, Liberati A, Tetzlaff J, Altman DG. Preferred reporting items for systematic reviews and meta-analyses: the PRISMA statement. BMJ. 2009;339:b2535.

11. Ogrinc G, Mooney SE, Estrada C, et al. The SQUIRE (Standards for QUality Improvement Reporting Excellence) guidelines for quality improvement reporting: explanation and elaboration. Qual Saf Health Care. 2008;17 Suppl 1:i13-32.

12. Wood R, Wilson P. General practitioner provision of preventive child health care: analysis of routine consultation data. BMC Fam Pract. 2012;13:73.

13. Auerbach AD, Wachter RM, Cheng HQ, et al. Comanagement of surgical patients between neurosurgeons and hospitalists. Arch Intern Med. 2010;170(22):2004-2010.

14. Kelly AM, Bryant M, Cox L, Jolley D. Improving emergency department efficiency by patient streaming to outcomes-based teams. Aust Health Rev. 2007;31(1):16-21.

15. Kim CS, Hayman JA, Billi JE, Lash K, Lawrence TS. The application of lean thinking to the care of patients with bone and brain metastasis with radiation therapy. J Oncol Pract. 2007;3(4):189-193.

16. Krening CF, Rehling-Anthony K, Garko C. Oxytocin administration: the transition to a safer model of care. J Perinat Neonatal Nurs. 2012;26(1):25-26.

17. Liss DT, Fishman PA, Rutter CM, et al. Outcomes among chronically ill adults in a medical home prototype. Am J Manag Care. 2013;19(10):e348-358. 
18. Murray O, Christen K, Marsh A, Bayer J. Fracture clinic redesign: improving standards in patient care and interprofessional education. Swiss Med Wkly. 2012;142:w13630.

19. Ravikumar TS, Sharma C, Marini C, et al. A validated value-based model to improve hospital-wide perioperative outcomes: adaptability to combined medical/surgical inpatient cohorts. Ann Surg. 2010;252(3):486-496.

20. Reid RJ, Fishman PA, Yu O, et al. Patient-centered medical home demonstration: a prospective, quasi-experimental, before and after evaluation. Am J Manag Care. 2009;15(9):e71-87.

21. Shannon RP, Frndak D, Grunden N, et al. Using real-time problem solving to eliminate central line infections. Jt Comm J Qual Patient Saf. 2006;32(9):479-487.

22. Shendell-Falik N, Feinson M, Mohr BJ. Enhancing patient safety: improving the patient handoff process through appreciative inquiry. J Nurs Adm. 2007;37(2):95-104.

23. Hwang TG, Lee Y, Shin H. Structure-oriented versus process-oriented approach to enhance efficiency for emergency room operations: what lessons can we learn? J Healthc Manag. 2011;56(4):255-267.

24. King DL, Ben-Tovim DI, Bassham J. Redesigning emergency department patient flows: application of Lean Thinking to health care. Emerg Med Australas. 2006;18(4):391-397.

25. Raab SS, Andrew-Jaja C, Condel JL, Dabbs DJ. Improving Papanicolaou test quality and reducing medical errors by using Toyota production system methods. Am J Obstet Gynecol. 2006;194(1):57-64.

26. Raab SS, Grzybicki DM, Condel JL, et al. Effect of Lean method implementation in the histopathology section of an anatomical pathology laboratory. J Clin Pathol. 2008;61(11):1193-1199.

27. Raab SS, Grzybicki DM, Sudilovsky D, Balassanian R, Janosky JE, Vrbin CM. Effectiveness of Toyota process redesign in reducing thyroid gland fine-needle aspiration error. Am J Clin Pathol. 2006;126(4):585-592.

28. Collar RM, Shuman AG, Feiner S, et al. Lean management in academic surgery. J Am Coll Surg. 2012;214(6):928-936.

29. Pennell L, Smith-Snyder CM, Hudson LR, Hamar GB, Westerfield J. Practice changes in glycemic management and outcomes in coronary artery bypass surgery patients. $J$ Cardiovasc Nurs. 2005;20(1):26-34.

30. Dimitrov DM, Rumrill PD, Jr. Pretest-posttest designs and measurement of change. Work. 2003;20(2):159-165.

31. Shojania KG, Grimshaw JM. Evidence-based quality improvement: the state of the science. Health Aff (Millwood). 2005;24(1):138-150.

32. Hulscher ME, Laurant MG, Grol RP. Process evaluation on quality improvement interventions. Qual Saf Health Care. 2003;12(1):40-46.

33. Nolte E, Conklin, A., Adams, J., Brunn, M., Cadier, B., Chevreul, K., Durand-Zaleski, I., Elissen, A., Erler, A., Flamm, M., Frolich, A., Fullerton, B., Jacobsen, R., Knai, C., Krohn, R., Pohlmann, B., Saz Parkinson, Z., Sarria Santamera, A., Sonnichsen, A., Vrijhhoef, H.,. Evaluating chronic disease management. Recommendations for funders and users. Santa Monica: RAND Corporation; 2012. 
34. Ovretveit J, Gustafson D. Evaluation of quality improvement programmes. Qual Saf Health Care. 2002;11(3):270-275.

35. Mazzocato P, Savage C, Brommels M, Aronsson H, Thor J. Lean thinking in healthcare: a realist review of the literature. Qual Saf Health Care. 2010;19(5):376-382.

36. Schouten LM, Hulscher ME, van Everdingen JJ, Huijsman R, Grol RP. Evidence for the impact of quality improvement collaboratives: systematic review. BMJ. 2008;336(7659):1491-1494.

37. Jackson GL, Powers BJ, Chatterjee R, et al. Improving patient care. The patient centered medical home. A Systematic Review. Annals of Internal Medicine. 2013;158(3):169-178.

38. Chalmers I, Glasziou, P., Godlee, F., All trials must be registered and te results published. Brit Med J. 2013;346:f105. 


\section{APPENDIX 1. SEARCH TERMS USED}

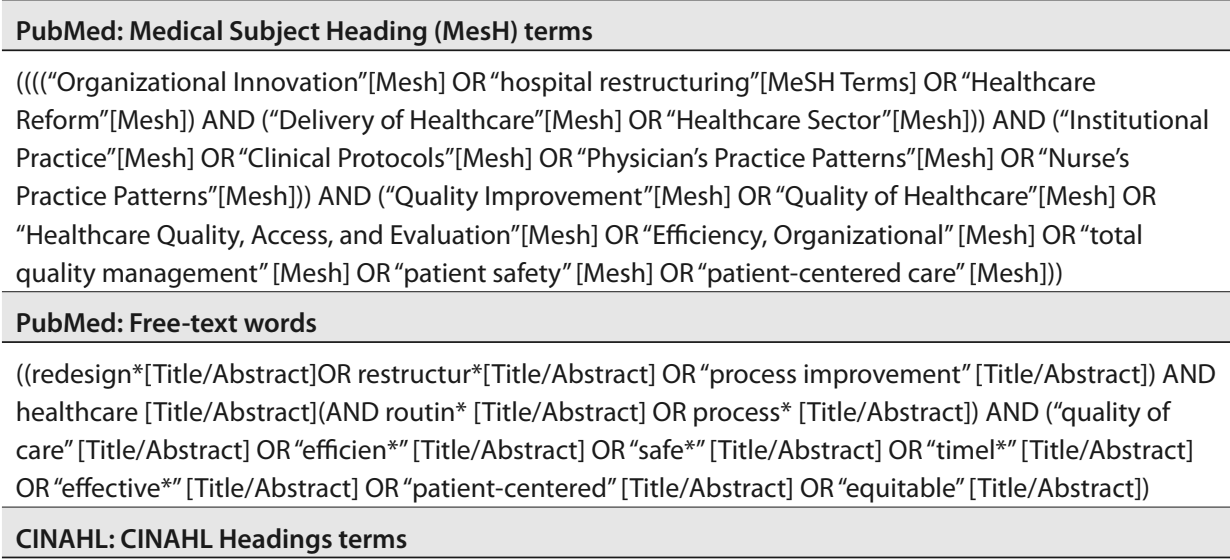

((MH “Work Redesign") OR (MH “Healthcare Reform”) OR (MH “Organizational Change") OR (MH "Organizational Restructuring")) AND (MH “Healthcare Delivery") AND ((MH "Medical Practice") OR (MH "Advanced Nursing Practice") OR (MH "Professional Practice, Research-Based") OR (MH "Professional Practice, Theory-Based") OR (MH "Nursing Practice") OR (MH "Professional Practice, Evidence-Based") OR (MH "Nursing Practice, Theory-Based") OR (MH "Nursing Practice, Research-Based") OR (MH "Nursing Practice, Evidence-Based") OR (MH "Medical Practice, Research-Based") OR (MH "Medical Practice, Evidence-Based") OR (MH “Nursing Care") OR (MH “Practice Patterns")) AND ((MH "Quality of Healthcare") OR (MH “Quality Management, Organizational”) OR (MH “Quality Assessment”) OR (MH “Quality Improvement") OR (MH “Quality Assurance") OR (MH "Quality of Nursing Care") OR (MH "Patient Safety") OR (MH “Organizational Efficiency") OR (MH "Patient Centered Care"))

\section{CINAHL: Free-text words}

(redesign* OR restructure* OR "process improvement") AND healthcare AND (routin* OR proces*) AND ("quality of care" OR efficiency OR safe* OR timel* OR effectiveness OR "patient-centered" OR equitable)

\section{Web of Science: Free-text words}

(redesign* OR restructure* OR "process improvement") AND healthcare AND (routin* OR proces*) AND ("quality of care" OR efficiency OR safe* OR timel* OR effectiveness OR "patient-centered" OR equitable)

\section{Business Premier Source: Thesaurus terms}

(((((DE “REENGINEERING (Management)") OR (DE “PROCESS optimization")) OR (DE "ORGANIZATIONAL change")) AND (DE "MEDICAL care")) AND (DE "ORGANIZATIONAL effectiveness"))

Business Premier Source: free-text words

(redesign* OR restructure* OR "process improvement") AND healthcare AND (routin* OR proces*) AND ("quality of care" OR efficiency OR safe* OR timel* OR effectiveness OR "patient-centered" OR equitable) 
The impact of redesigning healthcare processes 



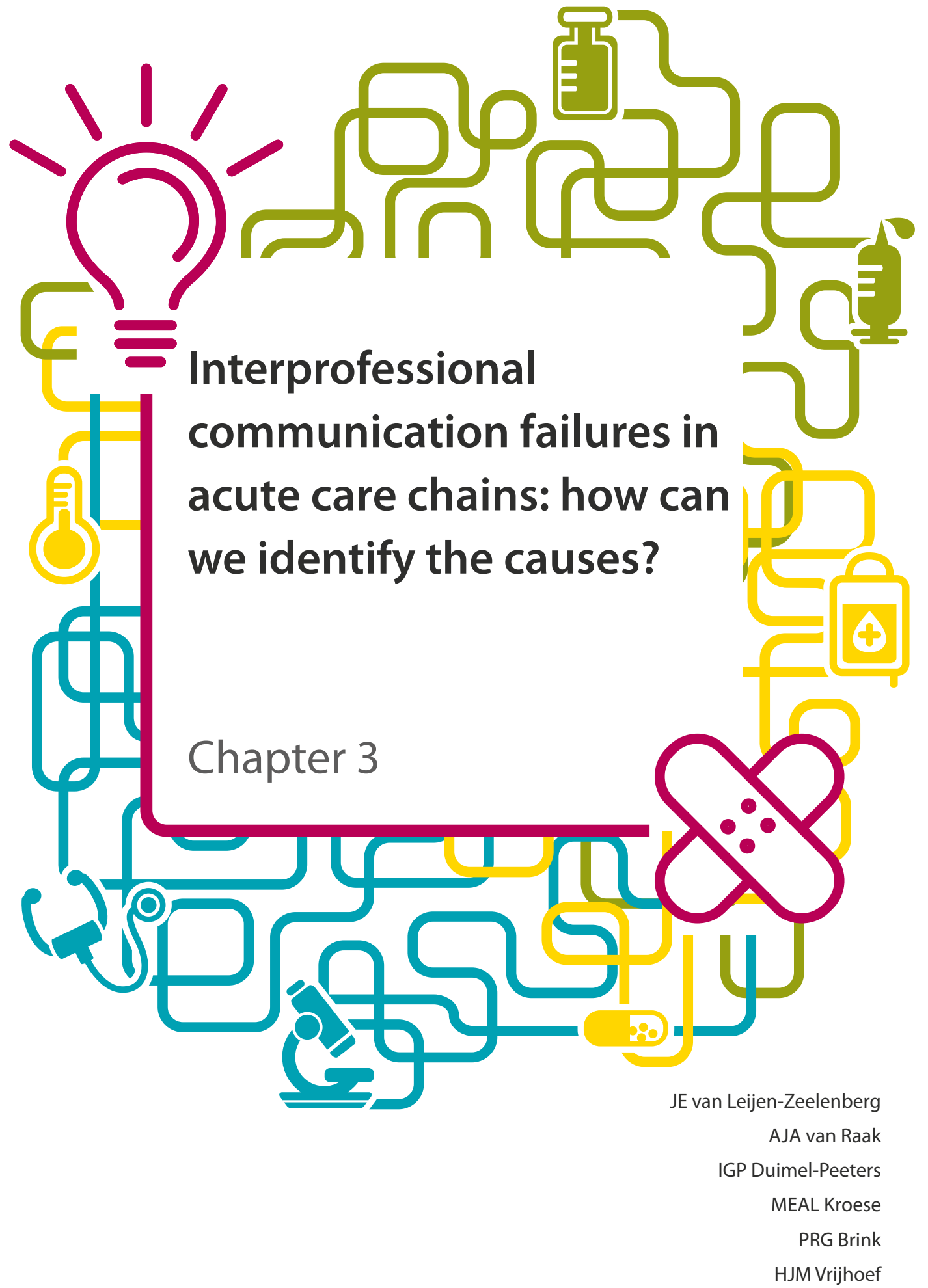

Journal of Interprofessional Care 2015, vol. 29, No. 4, Pages 320-330 


\title{
Chapter 3
}

\begin{abstract}
Although communication failures between professionals in acute care delivery occur, explanations for these failures remain unclear. We aim to gain a deeper understanding of interprofessional communication failures by assessing two different explanations for them. A multiple case study containing six cases (i.e. acute care chains) was carried out in which semi-structured interviews, physical artifacts and archival records were used for data collection. Data were entered into matrices and the pattern matching technique was used to examine the two complementary propositions. Based on the level of standardization and integration present in the acute care chains, the six acute care chains could be divided into two categories of care processes, with the care chains equally distributed among the categories. Failures in communication occurred in both groups. Communication routines were embedded within organizations and descriptions of communication routines in the entire acute care chain could not be found. Based on the results, failures in communication could not exclusively be explained by literature on process typology. Literature on organizational routines was useful to explain the occurrence of communication failures in the acute care chains. Organizational routines can be seen as repetitive action patterns and play an important role in organizations, as most processes are carried out by means of routines. The results of this study imply that it is useful to further explore the role of organizational routines on interprofessional communication in acute care chains to develop a solution for failures in handover practices.
\end{abstract}




\section{INTRODUCTION}

In 2000, the Institute of Medicine (IOM) focused attention to preventable errors in healthcare by publishing their report 'To Err is Human: building a safer Health System' ${ }^{\prime}$. The IOM reported that the emergency department was the location with one of the highest proportions of adverse events. Interprofessional communication failures are known to be the root cause of those adverse events ${ }^{2,3}$. More specifically, failures in handover communication are known to lead to interruptions in the continuity of care and have the potential to harm the patient - for instance by inappropriate treatments - and are therefore a threat to patient safety ${ }^{2,4-6}$. Other undesired consequences of failures in communication are increased patient complications; longer hospital stays; and lower patient satisfaction ${ }^{4,7-10}$. In acute care, communication often takes place in a busy and time-limited setting, challenging the professional ${ }^{4,11}$. As mistakes by professionals at the emergency department are often the result of poor communication, it is important to improve handover communication among professionals working in acute care $\mathrm{e}^{4,5,12}$.

In the Netherlands, growing attention is being paid to improving quality of acute care delivery. Acute care is increasingly organized in care chains, stimulated by the Dutch Ministry of Health, Welfare and Sports. In these acute care chains, care for patients with acute problems is delivered by multiple providers, with the aim to provide seamless care to patients with acute care needs ${ }^{13}$. Interprofessional collaboration structures - such as acute care chains - receive increasing attention in literature as they are considered to improve the quality of healthcare ${ }^{14-}$ ${ }^{16}$. Despite the growing body of literature promoting interprofessional collaboration however, challenges in interprofessional collaboration such as professional hierarchy or a lack of interprofessional feedback can prevent an interprofessional work system from achieving its goal in improving quality of care ${ }^{14-16}$. An exploratory Failure Mode and Effect Analysis carried out by the Network Acute Care Limburg (NAZL - in Dutch "Netwerk Acute Zorg Limburg") showed that it is apparent to healthcare providers in the acute care chains that communication failures exist and it is desired to evaluate the communication failures and assess where improvements can be made ${ }^{17}$. Factors that are considered to be of influence on (interprofessional) communication failures are amongst others the quality of leadership, the design of the care system and a lack of common understanding amongst an interprofessional team ${ }^{2,8,14,15,18,19}$. The Institute of Medicine addresses the importance of the design of the system and process characteristics in enhancing patient safety and in a broader perspective, emphasizing the need to redesign current healthcare systems in order to improve quality of care 1 . Understanding the design of the acute care chains can therefore be a first step towards gaining insight into the root causes of communication failures. Van Merode, Molema and Goldschmidt argue that analyzing healthcare processes by the degree of integration and standardization can be very useful in finding root causes for failures and creating opportunities for improvement ${ }^{20}$. However, since a lot of work or work processes are carried out through routines, using the concept of organizational routines to search for root causes of failures might also result in useful explanations ${ }^{21,22}$. Previous studies have shown that this concept can help explaining difficulties in interprofessional cooperation between healthcare ${ }^{23-25}$. 
We aim to gain a deeper insight into the causes for communication failures by translating the two aforementioned perspectives into the following propositions:

1. Failures in handover communication are linked to the type of care process, meaning that the more integrated and standardized a care process is, the fewer failures will occur ${ }^{20}$;

2. Failures in handover communication are caused by a lack of shared understandings about the organizational communication routine amongst providers, which results in differences in the way a routine is carried out ${ }^{26}$.

The two perspectives and propositions are rarely used in literature to explain the root causes of failures in handover communication and the insights into communication failures as derived by using these perspectives can be complementary to existing root cause analysis of communication failures in interprofessional settings.

\section{BACKGROUND}

The conceptual framing on which the two complementary propositions are based are described below.

\section{Process characteristics}

Characteristics of the clinical process are increasingly thought to be of influence to medical errors ${ }^{1}$. Besides errors like adverse events, characteristics of the clinical process might also influence errors in handover communication and can therefore be a source of explanation of communication failures. Van Merode, et al. ${ }^{20}$ state that two characteristics are of considerable importance on the quality of (care) processes, namely the degree of integration and the degree of standardization. Integration refers to the degree in which services are targeted to the patient's needs or - in cases of a low level of integration - to professional techniques used in healthcare. Standardization refers to the use of protocols and care processes with a minimum of variety and a high level of predictability. Analyzing processes based on their level of integration and standardization leads to a distinction of four processes, i.e. (1) procedure-based factories with highly standardized processes focused on professional techniques, (2) focused factories with high levels of standardization focused on the patient needs, (3) integrated care processes with low levels of standardization and focused on the needs of the patients, and (4) archipelagos with low levels of standardization and focused on professional techniques (see figure 1) 20,27. $^{2}$.

Processes with a low level of standardization (i.e. integrated care processes and archipelagos) have more decision points and each additional decision points weakens the process, as all types of errors - including communication errors - can occur at decision points. Thus, following Van Merode, errors - including communication failures - will more frequently occur in processes containing a high number of decision points (i.e. integrated care and archipelago structures).

If process characteristics are the root cause of communication failures in acute care chains, the acute care chains with a lower level of standardization should show a higher number of communication failures. 


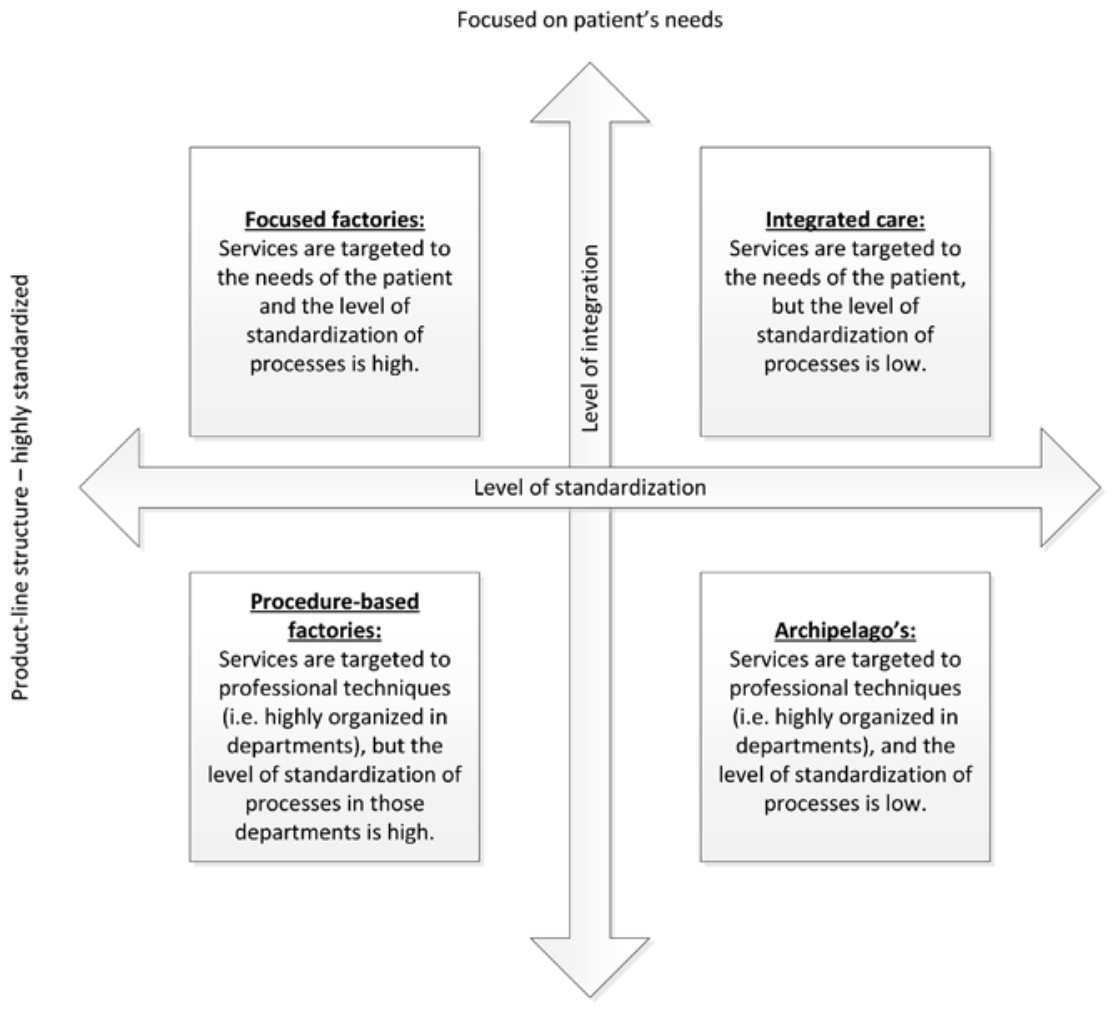

Focused on professional techniques

Figure 1 - Health care process arranged according to their characteristics (Van Merode, Molema, Goldshmidt, 2004)

\section{Organizational routines}

Organizational routines can be seen as repetitive action patterns and play an important role in organizations, as most processes are carried out by means of routines ${ }^{21,22,26,28}$. Using organizational routines as theoretical background to study problems in the delivery of interprofessional healthcare already showed the importance of routines in changing towards a new way of working. Discrepancies between the desired state of interprofessional cooperation and the emphasis on mono-professional organizational rules and routines for instance can hamper interprofessional cooperation ${ }^{23}$. Feldman \& Rafaeli ${ }^{26}$ noted that because carrying out organizational routines involves multiple individuals, these individuals will interact with each other and transfer information necessary to carry out the routine. Each connection that is created by doing so enables individuals to exchange information, which attributes to a shared understanding of the organizational routine and how the routine should be carried out. A lack of shared understanding can lead to either differences in how a routine is carried out or uncertainty about why a routine should be carried out. Applying this proposition to acute care chains, discrepancies in the way healthcare providers describe handover communication and discrepancies in existing artifacts or rules of involved organizations might be the cause of communication failures. 


\section{METHODS}

A multiple case study ${ }^{29}$ was carried out between October 2009 and April 2010 in the south of the Netherlands. The case study contained six cases i.e. acute care chains for: (a) cerebrovascular accidents (CVA), (b) acute myocardial infarction (AMI), (c) acute psychiatric care (APC), (d) acute obstetric care (AOC), (e) acute hip traumas (AHT) and (f) acute abdominal complaints (AAC). The selection of the cases was based on an assignment of the Dutch Ministry of Health, Welfare and Sports that was given to all acute care networks in the Netherlands. The university hospital in Maastricht is assigned by the Dutch Ministry of Health, Welfare and Sports to coordinate the organization of acute care in this region ${ }^{30}$. This coordination is realized by establishing a collaboration structure between the care providers (like hospitals, GPs, and ambulance care) - called the Netwerk Acute Zorg Limburg (NAZL, Dutch acronym for acute care network Limburg) who in turn make agreements on collaboration. These agreements attempt to lead to a seamless transition between each phase of acute care, in which the responsibilities of each care provider are clear and the patient receives the right care at the right place as soon as possible, ensuring continuity of acute care ${ }^{30}$. This is translated into acute care chains for five diagnosis categories (i.e. AMI, AHT, CVA, AOC, APC). Next to the five mentioned diagnosis categories, a sixth acute care chain $(A A C)$ was added in this region. All acute care chains existing in the research area were included in the case study as the authors were commissioned to analyze all six acute care chains.

\section{Study context}

In general, emergency care in the Netherlands is provided by General practitioners (GPs), midwives, (psychiatric) hospitals and ambulance care as most important healthcare providers. During office hours, all GPs are available for acute care; hence patients with acute care needs contact their own GP. Outside office hours however, patients with acute care needs should direct their care demand to the out of hour's primary care. The out of hours primary care (also called after-hours primary care) is a collaboration of GPs in a defined area which is available for primary care outside office hours and during the entire weekend ${ }^{31}$. A similar structure applies to psychiatric care; during office hours, patients with acute psychiatric care needs should direct their demand for care to their attending psychiatric care provider if already receiving treatment. Outside office hours however, patients with acute care needs should direct their demand to the out of hours primary care. For the AOC care chain, midwifes play an important role, as pregnancy check-ups are regularly carried out by midwifes (acting as independent profession rather than an in hospital service) and midwifes are therefore the first contact person of most patients.

\section{Data Collection}

Semi structured interviews, archival records and physical artifacts were used for data collection. These data collection techniques are among the commonly used data collection methods for case studies ${ }^{29}$. Combining three data collection techniques allowed us to cross examine the data (triangulation), enhancing validity and reliability of the findings ${ }^{29}$. Purposive sampling ${ }^{32}$ was used to select respondents for the semi structured interviews. Based on expert opinion, key 
providers in the six acute care chains were defined, leading to 48 respondents. Selection of these respondents was based on four selection criteria: (a) the respondent needed to work within the research area, (b) the respondent needed to work within the field of (at least) the acute care chain of subject and have knowledge of the specific acute care chain, (c) the respondents needed to show commitment to the research project and (d) for GPs specifically: the GP should have experience in working at the out of hours primary care. The names and addresses of the respondents were provided by the NAZL. Table 1 provides an overview of the characteristics of the respondents.

The semi-structured interview dealt with three major subjects; (a) the description of the care process of the acute care chain (providing data for the first proposition (i.e. process characteristics), (b) the description of the communication process within this acute care chain (providing data for the second proposition (i.e. organizational routines) and (c) strengths, weaknesses, opportunities and threats of communication and feedback. In total, the interview consisted of 22 questions. The respondents were asked to answer the questions for the specific acute care chain at which they worked. The time necessary to conduct an interview varied from 30 minutes to two hours - mainly due to available time and the length of answers given - with an average of one hour. Archival records describing the current situation of handover communication and characteristics and process descriptions of the six acute care chains were collected from a database of the Acute Care Network Limburg. Next to the archival records, physical artifacts were collected during the case study. Physical artifacts can be any physical records being gathered during observations, interviews or site visits ${ }^{29,33}$. For all care chains, physical artifacts e.g. handover communication forms or protocols describing handover communication - were collected during the semi-structured interviews.

\section{Data Analysis}

The pattern-matching technique ${ }^{29}$ - in which an empirically based pattern is compared to an already described one - was used as analytic approach. The two propositions were used as theoretical pattern and compared to the empirical pattern as found by data collection. Interviews were audio recorded and transcribed verbatim to increase validity. Data matrices ${ }^{32}$ were used for data analysis for all acute care chains. A template approach ${ }^{34}$ was chosen for the coding process with the codebook based on the key elements of the theory underlying the two propositions. These being (a) features of the acute care chain; (b) coordination; (c) information transfer where, what, when, whom, how; (d) feedback, (e) standardization - protocols, flowcharts, audits, other documents; (f) strengths of the acute care chain - tasks, responsibilities, coordination, information transfer, feedback; and (g) weaknesses of the acute care chain - tasks, responsibilities, coordination, information transfer, feedback. The 'bracketing' technique ${ }^{35}$ was used to fill the cells, firstly containing so called thick descriptions (i.e. literal interview passages) which were transformed into thin description(i.e. summarizing the respondents answers per concept) at a later stage. After entering interview data in these matrices, information from archival records and physical artefacts were entered into the same data matrices. 
Particularly, the researchers classified each acute care chain according to the presence or absence of standardization and integration and searched for communication errors within these processes. Likewise, the researchers then searched for discrepancies in handover communication routines between professionals and organizations and shared understandings of the communication routine amongst professionals and organizations. Pattern matching was done both within and across cases (i.e. the acute care chains). Table 2 provides an overview of the pattern matching technique used.

Data analysis was done independently by two researchers (JVL, AVR) and discrepancies between the researchers were solved through discussion until consensus was reached. Together with the use of multiple data collection techniques which allowed us to cross examine the data, reliability, validity as well as credibility and objectivity of the research findings was increased ${ }^{36}$.

\section{Ethical Considerations}

According to national regulation, full ethical approval was deemed unnecessary because participants in this study were not subject to any acts, nor were they forced to change their behavior at any point during the study ${ }^{37}$. The study was carried out in accordance with the standards of expected ethical behavior based on The Code of Ethics of the World Medical Association ${ }^{38}$. Anonymity was guaranteed to the respondents of the interviews and all respondents gave their consent for both the interview and the recording of the interview. 
Table 1 - Characteristics of interview respondents

\begin{tabular}{|c|c|c|c|c|c|}
\hline $\begin{array}{l}\text { Acute Care } \\
\text { Chain* }\end{array}$ & Discipline & \# interviews & $\begin{array}{l}\text { Response } \\
\text { rate }(\%)\end{array}$ & $\begin{array}{l}\text { \# years experience } \\
\text { in this role/ in total }\end{array}$ & Gender \\
\hline \multirow[t]{4}{*}{ 1. CVA } & GPs & 2 & 100 & $35 / 35+$ unkown & $M+M$ \\
\hline & ED Nurses & 2 & 100 & $22 / 22+3 / 3$ & $M+F$ \\
\hline & Ambulance caregivers & 2 & 100 & $10 / 10+22 / 22$ & $M+F$ \\
\hline & Medical specialists & 1 & 50 & $12 / 12$ & $\mathrm{~F}$ \\
\hline Total CVA & & 7 & 87.5 & Mean: 17.3/17.3 & $\begin{array}{l}\text { Male: } 4 \\
\text { Female: } 3\end{array}$ \\
\hline \multirow[t]{4}{*}{ 2. AMI } & GPs & 1 & 50 & $20 / 20$ & M \\
\hline & CCU Nurses & 2 & 100 & $30 / 30+25 / 30$ & $M+M$ \\
\hline & Ambulance caregivers & 2 & 100 & $8 / 8+6 / 24$ & $M+M$ \\
\hline & Medical specialists & 2 & 100 & $8 / 8+30 / 30$ & $M+M$ \\
\hline Total AMI & & 7 & 87.5 & Mean: 18.1/21.4 & $\begin{array}{l}\text { Male:7 } \\
\text { Female: } 0\end{array}$ \\
\hline \multirow[t]{4}{*}{ 3. $\mathrm{AO}$} & GPs & 1 & 50 & $25 / 25$ & M \\
\hline & Midwifes & 3 & 150 & $20 / 20+27 / 27+10 / 10$ & $\mathrm{~F}+\mathrm{F}+\mathrm{F}$ \\
\hline & Ambulance caregivers & 2 & 100 & $27 / 27+16 / 16$ & $M+F$ \\
\hline & Medical specialists & 2 & 100 & $14 / 14+29 / 29$ & $\mathrm{~F}+\mathrm{M}$ \\
\hline Total AO & & 8 & 100 & Mean: 21/21 & $\begin{array}{l}\text { Male: } 3 \\
\text { Female: } 5\end{array}$ \\
\hline \multirow[t]{4}{*}{ 4. AHT } & GPs & 1 & 50 & $10 / 18$ & M \\
\hline & ED Nurses & 2 & 100 & $10 / 13+5 / 5$ & $M+F$ \\
\hline & Ambulance caregivers & 1 & 50 & $14 / 14$ & M \\
\hline & Medical specialists & 2 & 100 & $13 / 13+$ unkown & $M+M$ \\
\hline Total AHT & & 6 & 75 & Mean: 10.4/12.6 & $\begin{array}{l}\text { Male: } 5 \\
\text { Female: } 1\end{array}$ \\
\hline \multirow[t]{4}{*}{ 5. APC } & GPs & 2 & 100 & $17 / 21+17 / 20$ & $\mathrm{~F}+\mathrm{M}$ \\
\hline & psychiatric Nurses & 1 & 50 & $40 / 40$ & M \\
\hline & Ambulance caregivers & 1 & 50 & $5 / 5$ & M \\
\hline & Medical specialists & 1 & 50 & $5 / 5$ & M \\
\hline Total APC & & 5 & 62.5 & Mean: 16.8/18.2 & $\begin{array}{l}\text { Male: } 4 \\
\text { Female: } 1\end{array}$ \\
\hline \multirow[t]{4}{*}{ 6. AAC } & GPs & 2 & 100 & $7 / 7+4 / 19$ & $M+M$ \\
\hline & ED Nurses & 2 & 100 & $5.5 / 5.5+3 / 3$ & $\mathrm{~F}+\mathrm{F}$ \\
\hline & Ambulance caregivers & 1 & 50 & $5 / 5$ & M \\
\hline & Medical specialists & 2 & 100 & Unkown + 11/11 & $\mathrm{F}+\mathrm{M}$ \\
\hline Total $A A C$ & & 7 & 87.5 & Mean: 4.6/8.4 & $\begin{array}{l}\text { Male: } 4 \\
\text { Female: } 3\end{array}$ \\
\hline $\begin{array}{l}\text { Total } \\
\text { interviews }\end{array}$ & & 40 & 83.3 & Mean: 14.7/16.5 & $\begin{array}{l}\text { Male: } 27 \\
\text { Female: } 13\end{array}$ \\
\hline
\end{tabular}

${ }^{*} \mathrm{CVA}=$ cerebrovascular accident, $\mathrm{AMI}=$ acute myocardial infarction, $\mathrm{AO}=$ acute obstetrics, $\mathrm{AHT}=$ acute hip traumas, $\mathrm{APC}=$ acute psychiatric care, $\mathrm{AAC}=$ acute abdominal complaints 


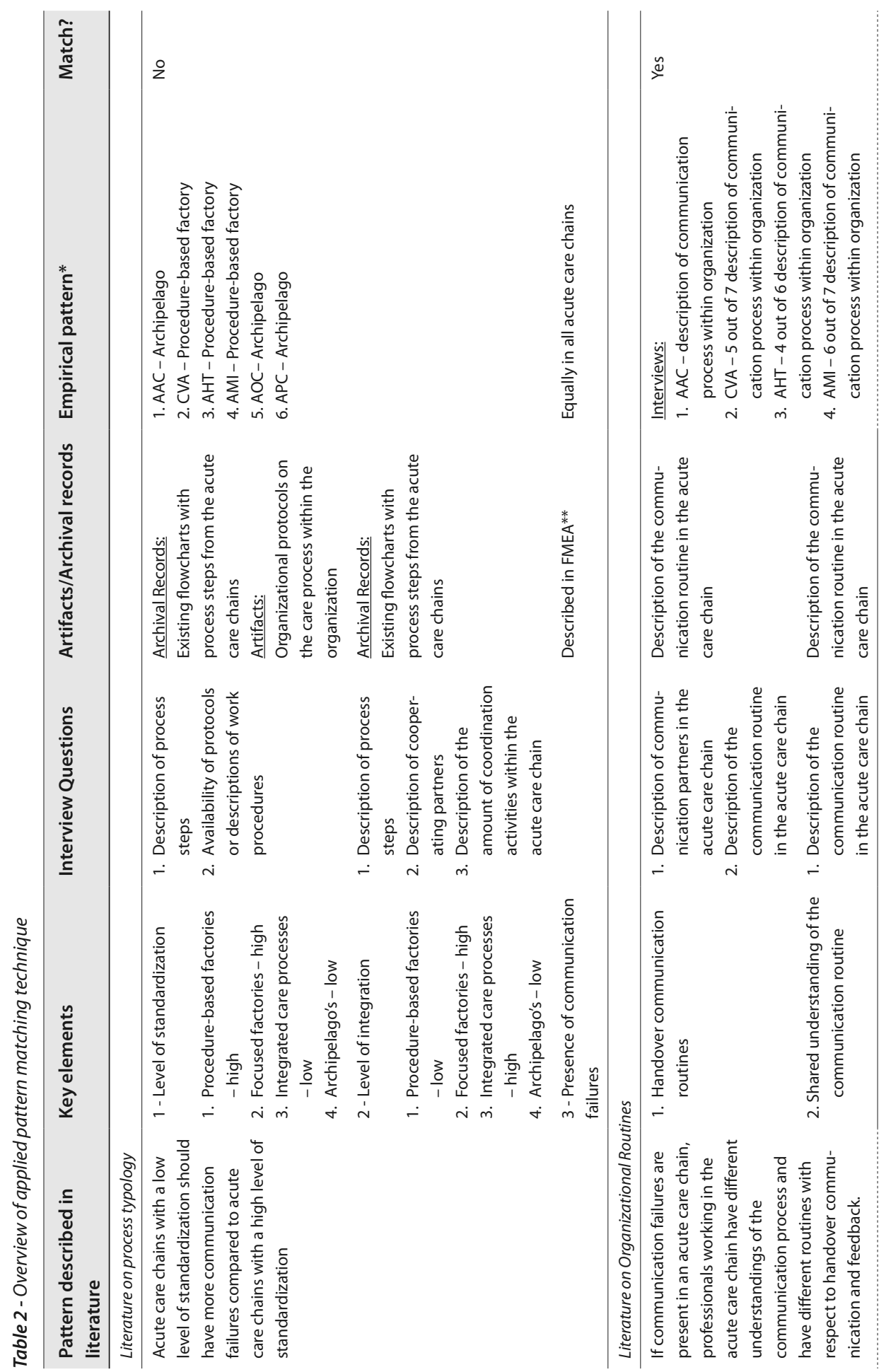


Interprofessional communication failures in acute care

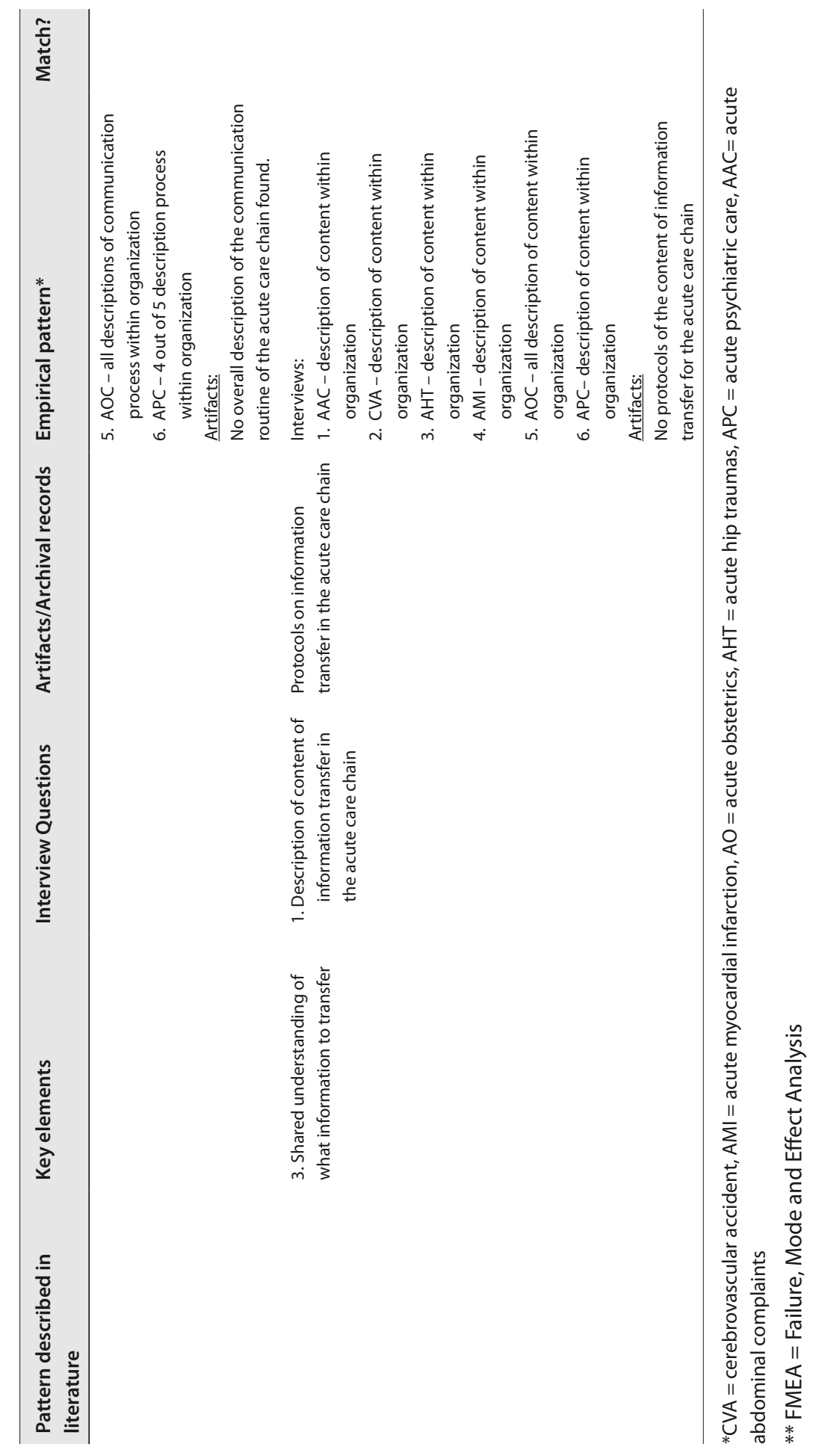




\section{RESULTS}

From the 48 respondents that were approached, 40 were interviewed (response rate $83.3 \%$ ). The response rates range from $62.5 \%$ (acute psychiatric care) to 100\% (acute obstetric care). From all provider categories, at least one was interviewed per acute care chain. Respondents were not obligated to provide reasons for non-response, however, most non-responders lacked time to participate in an interview, since the duration of the interview was approximately one hour and respondents were working in a busy and time limited healthcare setting. All interviews were held in the Dutch language and the quotes from the interviews as displayed here are translated into English. Archival records contained a Failure Mode and Effect Analysis (FMEA) of five acute care chains (CVA, myocardial infarction, acute hip trauma's, acute psychiatric care and acute obstetric care) performed by the Acute Care Network Limburg. In this FMEA, failures were described, with their possible causes and effects. Archival records also contained flowcharts describing five acute care chains (CVA, MCl, AHT, APC and AOC). No records existed on acute abdominal complaints. Most of the physical artifacts were forms for information transfer to other care providers in the acute care chain or protocols describing parts of the acute care chain.

\section{Communication failures}

A number of failures in handover communication were mentioned by providers from the different acute care chains (see Table 3). Failures that occurred in all acute care chains were a lack of feedback, a lack of structure of handover communication and an overall poor quality of handover communication. Most respondents clearly stated that providing feedback was uncommon in the acute care chains, but would be desirable.

Table 3 - Described communication failures in acute care chains

\begin{tabular}{ll}
\hline Communication failure & Acute Care Chain* \\
\hline Poor quality of handover & All \\
Handover too late & CVA, AHT, AAC \\
Handover lacks structure & All \\
Handover incomplete & CVA, AHT, AMI, AAC \\
Communication failures between specific care providers & AHT, AMI, AOC, APC, AAC \\
Lack of feedback & All \\
Poor coordination of care & AMI, AOC \\
\hline
\end{tabular}

* CVA = Cerebrovascular Accident; AHT= Acute Hip Traumas; AMI = Acute Myocardial Infarction; $\mathrm{AOC}=$ Acute Obstetric Care; $\mathrm{APC}=$ Acute Psychiatric Care; $\mathrm{AAC}=$ Acute Abdominal Complaints

"I think that GPs feel a strong need to get feedback about what happened with a patient after they referred him to the hospital." (GP1, AMI)

Sometimes, providers note that expectations on handover communication are unclear in the acute care chain: 
"To me it is unclear which information a psychiatrist desires from a GP, there is no fixed structure for that" (GP1, APC).

In general, all respondents indicated failures in handover communication.

\section{Process Characteristics}

The six cases and their process characteristics are described in Table 4. The six cases differed in the amount of standardization. However, all processes had a low level of integration, as care providers of different organizations functioned relatively solitarily in the acute care chains. Most respondents mention some form of fragmentation or experience difficulties in collaboration in the acute care chains.

"In the end we all work on separate islands, every organization has its own way of working, even though a regional protocol for CVA treatment exists" (Ambulance Nurse1, CVA).

In addition, respondents note that the way of working is based on one's own organization:

"We [midwifes and hospital] have different protocols and we don't always have up to date information on protocol changes and mutual expectations are also unclear sometimes". (Midwive1, AOC)

Highly standardized processes were seen in three care chains: for CVA, AMI and AHT. For CVA and $A M I$, protocols were available describing process steps and responsibilities of the different providers. For AHT, no protocols were available, but respondents could easily describe the acute care chain.

"The care chain is organized in a tight way; it is clear which people should be called or informed." (Nurse1, AHT)

For CVA, a nurse stated that

"The process lays down in protocols, within 4,5 hours from the development of the complaints, the patient should receive thrombolysis. (Nurse2, CVA)

In general, these processes were considered to be "easy" and "transparent" to the respondents and for every patient, similar process steps were described by the various respondents.

The three other studied processes were considered to be more diverse, depending on the presentation of complaints by the patient or the way a patient is referred within the acute care chain. As a result, carrying out each process step could lead to varying succeeding steps. According to a psychiatrist speaking about the process for APC:

"the route does not lay down in a protocol yet. Acute care does not function as a whole yet."(Psych1, APC)

indicating both a low level of standardization and integration. Respondents in the AOC care chain gave similar answers on these questions. A gynecologist indicated that protocols exist:

"depending on the problem that occurs, a few things are captured in flowcharts, but that is still under development since half a year." (Specialist 1, AOC) 
Table 4 - Characteristics of the six cases (i.e. acute care chains)

\begin{tabular}{llll}
\hline Acute care chain* $^{*}$ & Degree of integration & Degree of standardization & Organization type \\
\hline AAC & Low & Low & Archipelago \\
CVA & Low & High & Procedure-focused factory \\
AHT & Low & High & Procedure-focused factory \\
AMI & Low & High & Procedure-focused factory \\
AOC & Low & Low & Archipelago \\
APC & Low & Low & Archipelago \\
\hline
\end{tabular}

*CVA = cerebrovascular accident, $\mathrm{AMI}=$ acute myocardial infarction, $\mathrm{AO}=$ acute obstetrics,

$\mathrm{AHT}=$ acute hip traumas, $\mathrm{APC}=$ acute psychiatric care, $\mathrm{AAC}=$ acute abdominal complaints

\section{Organizational Routines}

Considering the routines of handover communication in the acute care chains, every organization seemed to have its own communication routines and a communication routine of the total acute care chain could not be defined. Respondents in different acute care chains found it difficult to explain the routine of the entire care chain in which they were involved, but could easily indicate the communication routine in their own organization. Hence, most respondents were only able to describe a fragment of the communication routine of the acute care chain in which they were involved (i.e. communication routine in their own organization).

"Communication mostly is about gearing activities from the referring partner to the emergency department. I wouldn't know what else is to be communicated." (Specialist 2, AAC)

About the communication routine in the APC care chain, a GP mentioned that

"We don't have a fixed structure for that, we transfer information orally.... I actually don't know what they [the psychiatric department] do. They probably make a record, but I don't make one for them." (GP2, APC)

A nurse working at the delivery room indicated that a communication routine on the level of the acute care chain does not exist:

"Communication highly depends on the situation, we do communicate with each other, but there is no structural way to do so" (Nurse1, AOC)

Mostly, the communication routine in the acute care chain was explained by mentioning communication methods (e.g. tools used to communicate with colleagues in the acute care chain or organization) or communication partners, mostly related to the organization in which the respondent worked. When respondents were asked to indicate the communication routine of the acute care chain, different respondents from the same organizations described similar routines within their organization, but only six out of 40 respondents (15\%) were able to describe the communication routine of the entire acute care chain (see Table 5). 
Table 5 - Description of organizational routines given by healthcare provider

\begin{tabular}{|c|c|c|c|c|}
\hline \multirow{2}{*}{$\begin{array}{l}\text { Acute Care } \\
\text { chain / } \\
\text { Healthcare } \\
\text { Provider* }\end{array}$} & \multicolumn{2}{|c|}{$\begin{array}{l}\text { \# providers being able to provide a } \\
\text { description of Care process }\end{array}$} & \multicolumn{2}{|c|}{$\begin{array}{l}\text { \# providers being able to provide a } \\
\text { description of communication process }\end{array}$} \\
\hline & $\begin{array}{l}\text { Organization } \\
\text { related }\end{array}$ & $\begin{array}{l}\text { Acute care chain } \\
\text { related }\end{array}$ & $\begin{array}{l}\text { Organization } \\
\text { related }\end{array}$ & $\begin{array}{l}\text { Acute care chain } \\
\text { related }\end{array}$ \\
\hline \multicolumn{5}{|c|}{ Acute Abdominal complaints } \\
\hline AT & 1 & & 1 & \\
\hline GP / GP HAP & 1 & 1 & 2 & \\
\hline Nurse ED & 2 & & 2 & \\
\hline Specialist ED & 2 & & 2 & \\
\hline \multicolumn{5}{|c|}{ Acute Cerebrovascular Accident } \\
\hline AT & 1 & 1 & 2 & \\
\hline GP / GP HAP & 2 & & 2 & \\
\hline Nurse ED & 1 & 1 & 1 & 1 \\
\hline Specialist ED & & 1 & & 1 \\
\hline \multicolumn{5}{|c|}{ Acute Hip traumas } \\
\hline AT & 1 & & 1 & \\
\hline GP / GP HAP & & 1 & 1 & \\
\hline Nurse ED & 2 & & 1 & 1 \\
\hline Specialist ED & 2 & & 1 & 1 \\
\hline \multicolumn{5}{|c|}{ Acute Myocardial Infarction } \\
\hline AT & 2 & & 2 & \\
\hline GP / GP HAP & 1 & & 1 & \\
\hline Nurse CCU & 1 & 1 & 1 & 1 \\
\hline Specialist CCU & 1 & 1 & 2 & \\
\hline \multicolumn{5}{|c|}{ Acute Obstetric Care } \\
\hline AT & 2 & & 2 & \\
\hline Midwife & & 1 & 1 & \\
\hline GP /GP HAP & 1 & & 1 & \\
\hline Nurse OD & 2 & & 2 & \\
\hline Specialist OD & 2 & & 2 & \\
\hline \multicolumn{5}{|c|}{ Acute Psychiatric Care } \\
\hline AT & 1 & & 1 & \\
\hline GP / GP HAP & 2 & & 2 & \\
\hline Nurse PED & 1 & & 1 & \\
\hline Specialist PED & 1 & & & 1 \\
\hline
\end{tabular}

* AT= Ambulance Team; HAP= out of hour's primary care (Dutch: Huisartsenpost); ED= Emergency

Department; CCU: Cardiac Care Unit; OD= Obstetrics Department; PED: Psychiatric Emergency Department 


\section{DISCUSSION}

Two types of care processes were distinguished in this study; procedure-based factories (high level of standardization, low level of integration) and archipelago's (low level of standardization, low level of integration). Failures in handover communication were indicated in all acute care chains, thus failures occur regardless of the level of standardization of an acute care chain. The pattern found by data collection therefore does not match the pattern described in literature. This implies that higher levels of standardization, by means of the presence of protocols and standardized work procedures, in an acute care chain do not prevent communication from failures. However, it should also be noted that most protocols or standardized work procedures were present at organizational level - with the exception of the CVA protocol. Standardization of work processes only within organizations involved in an acute care chain only is therefore unlikely to prevent communication failures.

Results indicated that shared understandings of the communication routine on the level of the acute care chain are not yet developed, or are very weak. Respondents were only able to indicate communication routines within their organization and physical artifacts were also organizationbased (as displayed by hospital based protocols or specific midwifes protocols for instance). Consequences of this lack of shared understanding can occur at two levels according to Feldman \& Rafaeli ${ }^{26}$; either the care providers in the acute care chain have (a) different understandings on what tasks they have to perform or (b) on why the routine is carried out ${ }^{26}$. Accordingly, in the acute care chains, care providers might have different understandings on what and how to communicate in the acute care chain and/or why the communication routine should be carried out. This is also in line with the types of communication failures that were described the most (i.e. handover communication being unstructured and of poor quality)

In the end, when healthcare providers do not share understandings on the communication routine, this will lead to differences in the way information is being transferred in the acute care chain or in what information is being transferred and to whom. The communication routines which the providers hold on to are embedded within their organization and might conflict with communication routines of other organizations involved in the same acute care chain.

Amongst others, effective communication is described in literature to be a core competency for collaborative practice which is of considerable complexity as effective communication has multiple components ${ }^{9}$. Improvement of communication skills however, is perceived to positively influence the quality of patient care ${ }^{9,39}$. Literature on organizational routines shows promising results in better understanding a variety of healthcare related problems $s^{23-26,40}$, but focus is not yet on communication in (acute) care chains. In addition, the importance of building shared understandings through open communication is described earlier in an OR (Operating Room) setting ${ }^{41}$. Studies evaluating the effect of communication tools like SBAR - Structure, Background, Assessment and Recommendation ${ }^{42}$ and SCRIPT - Structuring Communication Relationships for Interprofessional Teamwork ${ }^{43}$ aim to improve effectiveness of information transfer between healthcare providers by means of standardized communication procedures 
and focusing on core competencies of collaborative communication. Our results add to existing literature that discrepancies in organizational routines between collaborating professionals can be of influence on the quality of handover communication in acute care chains.

As this study was conducted in the south of the Netherlands, generalization of findings may be limited. Routines lay down in organizational rules, which can differ from one organization to another. Results and implications may therefore differ from one organization to another, stressing the importance of organizational features. Organizational rules can also exist on the level of acute care chains, and may therefore also differ from one care chain to another. Hence, not only features of the organization but also features of care chains should be acknowledged. However, acute care chains are described according to a national assignment, implying that the cases being studied here are to a high extent comparable to the other acute care chains in the Netherlands. More generally, context-specific data collection is inherent to case study research, as a specific context is the object of study ${ }^{29}$. In addition, case study research is important to develop a nuanced understanding of the real-life context in which theories are applied ${ }^{44}$. Applying the two theoretical propositions to more cases both nationally and internationally (i.e. replication) would increase the external validity of the finding ${ }^{29}$.

The number of participants ( $n=40$ ) can be seen as a limitation of this study, however purposive sampling was used to address this problem. In addition, as respondents within organizations indicated similar communication patterns, variability of findings in a larger group of respondents in the same organizations can be doubted. Moreover, the emphasis of this study focused upon examining two complementary theoretical propositions for failures in handover communication in acute care chains, rather than to create a general understanding. A case study is a suitable research method for this purpose (Yin, 2009). By limiting the analysis to using two propositions, the findings of this study do not reflect all causes of communication failures. Within the interprofessional literature, factors like professional hierarchy, the ability to build trusting relationships with other professions and the absence of electronic communication tools amongst others, are mentioned to be of influence on effective collaborative communication ${ }^{14,15,18,19}$. The findings of this study remain important however, as discrepancies in organizational routines as causes of communication failures indicate a need to pay attention to changing routines in improving collaborative communication. In addition, some studies already pointed routines to act as a barrier for improvement ${ }^{15,45}$. To increase validity of the findings of this study data was cross examines and data was analyzed independently by two reviewers, using a predefined codebook.

\section{CONCLUDING COMMENTS}

In addition to existing literature which describes the influence of leadership, professional hierarchy, building trusting relationships and other factors to be of influence on collaborative practice and communication, this study adds that organizational routines can also be of influence on interprofessional communication. Accordingly, the results imply attempts focused 
on solving the existing failures in handover communication should not only focus on structuring communication and prescribing communication methods, but also consider connecting care providers within the acute care chains. The ultimate goal would be to create a strong communication network between the care providers in the acute care chains, ensuring a shared understanding on how communication in the care chains should be carried out. As a communication network is established by the existence of connections between individuals involved in the routine ${ }^{26}$, the first step would be to closely explore all connections in the acute care chain and search for the exact discrepancies in interprofessional communication routines involved in the acute care chain. In addition, since professionals in the acute care chains use organization based communication routines, the lack of shared understanding should be evaluated (e.g. what are the expectations of the involved professionals with regards to what, how and to whom information should be transferred). Ultimately, as the study of Kerber et al. ${ }^{39}$ indicated, an improved approach to communication will lead to enhanced quality of care in acute care chains. Finally, if taking into account contextual characteristics, literature on organizational routines might lead to promising understanding in other fields of healthcare where two or more organizations cooperate and flaws in the communication process exist.

\section{DECLARATION OF INTEREST}

The authors declare no conflicts of interest. The authors are responsible for the writing and content of this paper. 


\section{REFERENCES}

1. Institute of Medicine. To Err is Human: Building a Safer Health System. Washington D.C.: Institute of Medicine;2000.

2. Cheung DS, Kelly JJ, Beach C, et al. Improving handoffs in the emergency department. Ann Emerg Med. 2010;55(2):171-180.

3. Patterson ES, Roth EM, Woods DD, Chow R, Gomes JO. Handoff strategies in settings with high consequences for failure: lessons for health care operations. Int J Qual Health Care. 2004;16(2):125-132.

4. Eisenberg EM, Murphy AG, Sutcliffe K, et al. Communication in Emergency Medicine: Implications for Patient Safety This Study was funded by a generous grant from the National atient Safety Foundation. Communication Monographs. 2005;72(4):390-413.

5. Kesavan S, Kelay T, Collins RE, et al. Clinical information transfer and data capture in the acute myocardial infarction pathway: an observational study. J Eval Clin Pract. 2013;19(5):805-811.

6. WHO Collaborating Centre for Patient Safety Solutions. Communication During Patient HandOvers. Patient Safety Solutions. 2007;1(3).

7. Collins SA, Mamykina $L$, Jordan $D$, et al. In search of common ground in handoff documentation in an Intensive Care Unit. J Biomed Inform. 2012;45(2):307-315.

8. Kripalani S, LeFevre F, Phillips CO, Williams MV, Basaviah P, Baker DW. Deficits in communication and information transfer between hospital-based and primary care physicians: implications for patient safety and continuity of care. Jama. 2007;297(8):831-841.

9. Suter E, Arndt J, Arthur N, Parboosingh J, Taylor E, Deutschlander S. Role understanding and effective communication as core competencies for collaborative practice. J Interprof Care. 2009;23(1):41-51.

10. Waibel S, Henao D, Aller MB, Vargas I, Vazquez ML. What do we know about patients' perceptions of continuity of care? A meta-synthesis of qualitative studies. Int J Qual Health Care. 2012;24(1):39-48.

11. Bost N, Crilly J, Wallis M, Patterson E, Chaboyer W. Clinical handover of patients arriving by ambulance to the emergency department - a literature review. Int Emerg Nurs. 2010;18(4):210-220.

12. Beach C, Croskerry P, Shapiro M. Profiles in patient safety: emergency care transitions. Acad Emerg Med. 2003;10(4):364-367.

13. Gijsen R, Kommer GJ, Bos N, van Stel H. Hoe is acute zorg georganiseerd? In: Volksgezondheid Toekomst Verkenning, Nationaal Kompas Volksgezondheid 2010; Derived from: http://www.nationaalkompas.nl>NationaalKompas Volksgezondheid\Zorg\Sectoroverstijgend\Acutezorg, 2010.

14. Reeves S, Rice K, Conn LG, Miller KL, Kenaszchuk C, Zwarenstein M. Interprofessional interaction, negotiation and non-negotiation on general internal medicine wards. J Interprof Care. 2009;23(6):633-645.

15. Rice K, Zwarenstein M, Conn LG, Kenaszchuk C, Russell A, Reeves S. An intervention to improve interprofessional collaboration and communications: a comparative qualitative study. J Interprof Care. 2010;24(4):350-361. 
16. Zwarenstein M, Reeves S. Working together but apart: barriers and routes to nurse--physician collaboration. Jt Comm J Qual Improv. 2002;28(5):242-247, 209.

17. Traumacentrum Limburg. Failure Mode and Effects Analysis CVA, Myocardinfarct, Heuptrauma, Obstetrie, Psychiatrie. Maastricht: Traumacentrum Limburg; 2009.

18. Chatalalsingh C, Reeves S. Leading team learning: what makes interprofessional teams learn to work well? J Interprof Care. 2014;28(6):513-518.

19. Zwarenstein M, Rice K, Gotlib-Conn L, Kenaszchuk C, Reeves S. Disengaged: a qualitative study of communication and collaboration between physicians and other professions on general internal medicine wards. BMC Health Serv Res. 2013;13:494.

20. Van Merode F, Mollema H, Goldschmidt H. GUM and six sigma approaches positioned as deterministic tools in quality target engineering. Accreditation and Quality Assurance. 2004;10:32-36.

21. Becker MC. Organizational Routines: a review of the literature. Industrial and Corporate Change. 2004;13(4):634-677.

22. Pentland BT, Feldman MS. Organizational routines as a unit of analysis. Industrial and Corporate Change. 2005;14(5):793-815.

23. Elissen AMJ, Van Raak A, Paulus A. Can we Make sense of multidisciplinary co-operation in primary care by considering routines and rules? Heatlh and Social Care in the community. 2010.

24. Van Raak A, Groothuis S, van der Aa R, Limburg M, Vos L. Shifting stroke care from the hospital to the nursing home: explaining the outcomes of a Dutch case. J Eval Clin Pract. 2010;16(6):1203-1208.

25. Van Raak A, Paulus A, Cuijpers R, te Velde C. Problems of integrated palliative care: a Dutch case study of routines and cooperation in the region of Arnhem. Health Place. 2008;14:768-778.

26. Feldman MS, Rafaeli A. Organizational Routines as sources of connections and understandings. $J$ Manage Stud. 2002;39(3):309 - 331.

27. Van Merode F. A prelude of the 2004 Antwerp Quality Conference: Targets and target values - integrating quality management and costing. Accreditation and Quality Assurance. 2004;9:168-171.

28. Van Raak A, Paulus ATG, Groothuis S. Integrated Care Delivery: Process Redesign and the Role of Rules, Routines and Transaction Costs. In: Klein LA, Neumann EL, eds. Integrated Heatlh Care Delivers. New York: Nova Science Publishers; 2008:115-135.

29. Yin RK. Case Study Research: Design and Methods Vol 5. 4 ed. Thousand Oaks: SAGE Inc.; 2009.

30. Traumacentrum Limburg. Traumacentrum Limburg: Wat zijn onze taken? 2011; http://www.azm.nl/ zorgcentra/zorgcentra/traumazorg/werkgebied/taken. Accessed 14-04-2011, 2011.

31. Giesen P, Smits M, Huibers L, Grol R, Wensing M. Quality of After-Hours Primary Care in the Netherlands: A Narrative Review. Annals of Internal Medicine. 2011;155(2):108-U196.

32. Miles M, Huberman M. Qualitative data analysis: An expanded sourcebook (2nd ed.). Thousand Oaks, CA: Sage; 1994.

33. Tellis W. Application of a Case Study Methodology. The Qualitative Report. 1997;3(3):1. 
34. Fereday J, Muir-Cochrane, E., . Demonstrating rigor in thematic analysis: A hybrid approach of inductive and deductive coding and theme development. International Journal of Qualitative Methods. 2006;5(1):80-92.

35. Denzin NK. Interpretive Interactionism. Newbury Park: Sage Publications; 1989.

36. Eisenhardt KM. Building Theories from Case Study Research. Academy of Management Review 1989;14(4):532-550.

37. Ministerie van Volksgezondheid, Welzijn en Sport. Wet medisch-wetenschappelijk onderzoek met mensen. BWBR00094082014. Den Haag: 2014.

38. World Medical Association. Declaration of Helsinki, Ethical Principals for Medical Research Involving Human Subjects. 2013.

39. Kerber KA, Hofer TP, Meurer WJ, Fendrick AM, Morgenstern LB. Emergency department documentation templates: variability in template selection and association with physical examination and test ordering in dizziness presentations. BMC Health Serv Res. 2011;11:65.

40. Zisberg A, Young HM, Schepp K, Zysberg L. A concept analysis of routine: relevance to nursing. J Adv Nurs. 2007;57(4):442-453.

41. Gillespie BM, Gwinner K, Chaboyer W, Fairweather N. Team communications in surgery - creating a culture of safety. J Interprof Care. 2013;27(5):387-393.

42. Dunsford J. Structured communication: improving patient safety with SBAR. Nurs Womens Health. 2009;13(5):384-390.

43. Zwarenstein M, Reeves S, Russell A, et al. Structuring Communication Relationships for Interprofessional Teamwork (SCRIPT): a cluster randomized controlled trial. Trials. 2007;8:23.

44. Flyvbjerg B. Five Misunderstandings about Case-Study Research. Qualitative Inquiry. 2006;12(2):219-245.

45. van Leijen-Zeelenberg JE, van Raak AJ, Duimel-Peeters IG, et al. Barriers to implementation of a redesign of information transfer and feedback in acute care: results from a multiple case study. BMC Health Serv Res. 2014;14:149. 



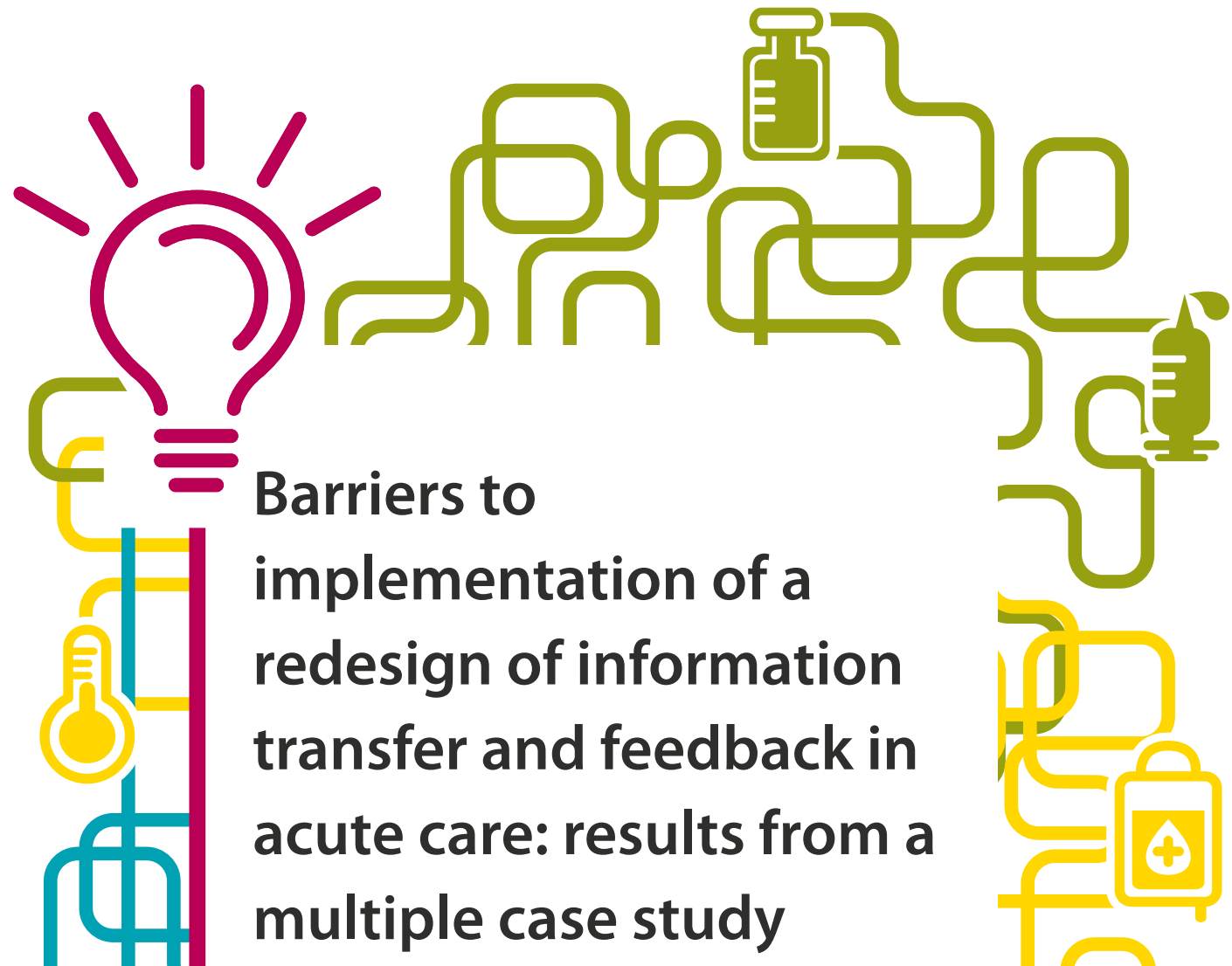

Chapter 4

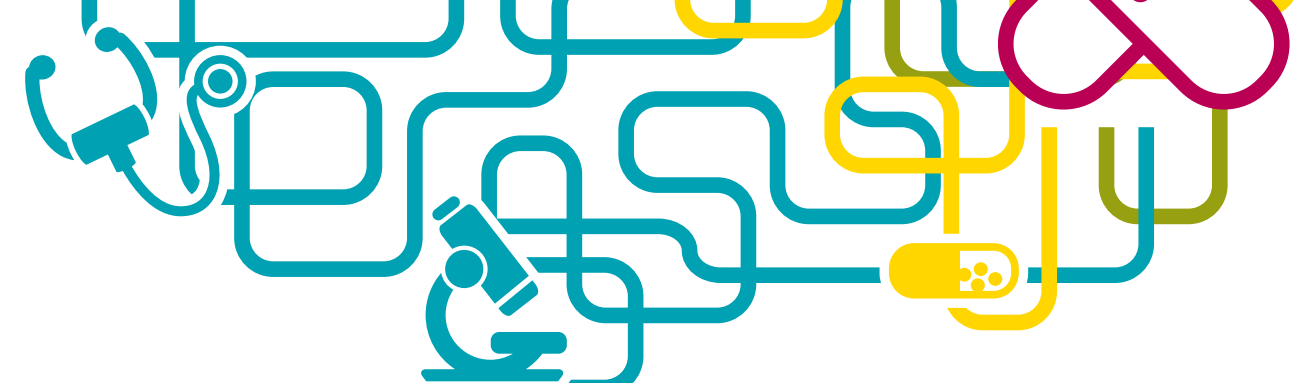

JE van Leijen-Zeelenberg AJA van Raak IGP Duimel-Peeters MEAL Kroese PRG Brink D Ruwaard

HJM Vrijhoef BMC Health Services Research, 14: 149 (2014) 


\section{ABSTRACT}

Background: Accurate information transfer is an important element of continuity of care and patient safety. Despite the demonstrated urge for improvement of communication in acute care, there is a lack of data on improvements of communication. This study aims to describe the barriers to implementation of a redesign of the existing model for information transfer and feedback.

Methods: A case study with six cases (i.e. acute care chains), using mixed methods was carried out in the Netherlands. The redesign was implemented in one acute care chain while the five other acute care chains served as control groups. Focus group interviews were held with members of the acute care chains and questionnaires were sent to care providers working in the acute care chains.

Results: Respondents reported three sets of barriers for implementation of the model: (a) existing routines for information transfer and feedback in organizations within the acute care chain; (b) barriers related to the implementation method and time period; and (c) the absence of a high 'sense of urgency' amongst providers in the acute care chain which would aid in improving the communication process.

Conclusions: This study shows that organizational factors play an important role in the success or failure of redesigning a communication process. Organizational routines can hamper implementation of a redesign if it differs too much from the routines of care providers involved. Besides focusing on provider characteristics in the implementation of a redesigned process, specific attention should be paid to unlearning existing organizational routines.

Keywords: Communication, Redesign, Barriers, Implementation, Acute care, Emergency Care, Healthcare providers 


\section{BACKGROUND}

Accurate communication is an important feature of seamless care and enhances patient safety $^{1-4}$. Information transfer and patient handovers are noted to be potentially hazardous areas for error in emergency care. Failures in information transfer between healthcare professionals can lead to several errors in care processes, such as poor coordination, inefficient functioning of healthcare providers and longer waiting and throughput times for patients ${ }^{5,6}$. In emergency care, communication failure is known to be the root cause of most adverse events ${ }^{1,7}$. Improving communication in emergency care is therefore necessary, although the subject has received relatively little attention and published studies are of variable quality ${ }^{1,4,5,7,8}$. In the Netherlands, emergency care is partially organised by means of acute care chains. An acute care chain can be defined as the description of the patient flow of a specific diagnosis category, in need of acute care, including agreements on the responsibilities of the healthcare providers involved ${ }^{9}$. Despite efforts of cooperation in a care chain, healthcare providers mention the presence of bottlenecks in communication such as shortage of information on the patients' case or absence of feedback ${ }^{9}$. A redesign of the communication process, focusing on information transfer and feedback was developed, however implementation of the redesign failed (See Methods: Implementation). The aim of this study was to understand the barriers to implementation of the redesign. The main research question addressed in this article therefore is: What barriers to implementation of redesign of acute care in the Maastricht Heuvelland area are perceived by healthcare providers?

On its turn, understanding barriers to implementation helps to create a solid understanding of efforts necessary to improve communication in emergency care. Hence, even in broader perspective, there is a need to prevent under-reporting of research results ${ }^{10}$. In addition, this study also aims to provide information to overcome the existing knowledge gap in improving communication in emergency care.

\section{METHODS}

Because of the explorative nature of the research question, a multiple case study comprising six cases, using mixed methods was carried out between October 2009 and April 2011 in the Netherlands. The redesign was implemented in the acute care chain for acute abdominal complaints (AAC) with the five other acute care chains serving as control groups.

\section{Intervention}

The redesign for information transfer and feedback aimed to improve information transfer in two ways (Figure 1):

1. A standardized electronic referral form to be used between all healthcare providers involved in the acute care chain should ensure availability of the right information for the right healthcare provider, in the right format at the right time.

2. A standardized feedback form to be used between all healthcare providers involved in the acute care chain should help the healthcare providers to continuously improve the 
quality of information transfer. The healthcare provider can state his or her preferences for feedback at each referral in the acute care chain and feedback can be requested on information transfer, medical performance, the care process and the referral.

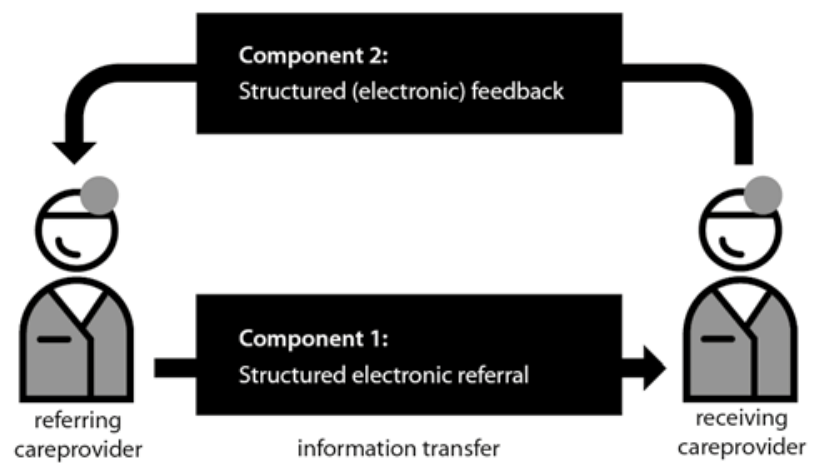

Figure 1 - Redesign for information transfer and feedback

Literature on information transfer and feedback ${ }^{11-14}$ combined with input from healthcare providers working in acute care chains served as input for the redesign. Three consensus meetings with professionals of the acute care chain for AAC were held in order to develop the redesign. A researcher (JVLZ) chaired these meetings and provided the professionals with adjusted versions of the redesign.

\section{Implementation}

For the implementation of the redesign an implementation group was established. This group consisted of an implementation group leader (PB), a content related coordinator, a logistical coordinator (JVLZ), and five opinion leaders active in the acute care chain for AAC. Being involved at the management level in the trauma center, the implementation group leader was considered to be an opinion leader. The opinion leaders were considered capable to influence the other healthcare professionals working in the acute care chain for AAC, as they were either positioned at an intermediate management level of one of the organizations involved or were considered to be experts on the field of AAC. Implementation activities consisted of the introduction of the redesign to management of the Emergency Care unit and its staff, the ambulance staff, the GPs and staff working at the out-of-hours primary care service (OHPCS). These groups were repeatedly informed of the implementation and use of the redesign, by means of newsletters, emails and presentations. During the implementation period (June 2010 - January 2011), the implementation group held monthly meetings in which goals were set and activities were defined and adjusted based on an intermediate evaluation. The redesign of the information transfer and feedback was hardly implemented, if at all. The first component of the redesign (i.e. the structured electronic referral) was not implemented and the second component of the redesign (i.e. the structured feedback) was implemented but sporadically used. The feedback form was implemented in paper form, and of the 80 forms distributed in the care chain, only 
four were used. Of these four forms, one was used accurately, one was ripped apart and had to be corrected and replaced and two were not filled in properly.

\section{Research area}

The selection of cases follows from an assignment of the Dutch Ministry of Public Health, Sports and Welfare of the 11 regional trauma centers in which five acute care chains are defined. These being: (a) cerebrovascular accidents (CVA), (b) acute myocardial infarction (AMI), (c) acute hip traumas (AHT), (d) acute psychiatric care (APC) and (e) acute obstetric care (AOC). All of these acute care chains were included in the case study. Located in the Maastricht Heuvelland $(\mathrm{MH})$, other important features of the case study include (1) the existence of a sixth acute care chain - for AAC, (2) the OHPCS located next to the emergency department, (3) in some cases, ambulances from an ambulance base other than Maastricht take care of the transport of patients and (4) the psychiatric hospital located in Maastricht being responsible for hospital care delivery to patients of the $\mathrm{MH}$ area with acute psychiatric complaints outside office hours. The OHPCS 'Maastricht Heuvelland' is a collaboration of GPs in MH and works during evening, night and weekend shifts ${ }^{15,16}$.

\section{Data Collection}

Following the implementation period, written questionnaires and focus group (FG) interviews were used to determine perceived barriers to implementation of the redesign. Purposive sampling ${ }^{17}$ was used to select respondents for the questionnaires representative for the entire acute care chain. Initially four key categories of healthcare providers were selected; (a) Ambulance caregivers, (b) General Practitioners (GPs), (c) Nurses from the Emergency Department and (d) Medical specialists working at the Emergency Department. These four categories were considered to represent the main disciplines and professionals involved in an acute care chain. The acute care chains for acute psychiatrics, acute myocardial infarction and acute obstetrics, however, are organized in such a way that the Emergency Department is only indirectly involved in these acute care chains. For these acute care chains, the disciplines (a) psychiatry nurse, (b) psychiatrist, (c) nurses from the Cardiac Care Unit, (d) medical specialists working at the Cardiac Care Unit, (e) midwives, (f) nurses from the obstetric unit and (g) medical specialists working at the obstetrics unit respectively were added.

In total, 40 respondents were approached. In the questionnaires respondents were shown 14 characteristics of the acute care chains. For each characteristic, respondents were asked to indicate - on a five-point scale - in what manner they thought the characteristic would influence the quality of information transfer and feedback (see Table 4). Two further questions asked respondents whether they perceived improvement in information transfer and feedback to be important. FG interviews were planned for all acute care chains (size of the groups ranged from three to fourteen participants), with an aim to determine perceived barriers to implementation. FG interviews were planned during regular meetings of the coordinating group of the acute care chain. A coordinating group consists of representatives of all care providers involved in an 
acute care chain and were therefore considered to be an optimal representation of the acute care chains. Because we used the coordinating group of the care chains for the FG interviews, we did not balance in age, work experience or hierarchical status of the respondents. Therefore, although participants were considered to be representative for the acute care chains, the composition of the FGs might have caused some bias. Not all acute care chains had such a coordinating group however. In addition, a FG interview was planned with the implementation group. All FG interviews were moderated by a researcher (JVLZ). Before the start of the FG interviews the moderator ensured anonymity and all participants gave their consent.

The FG interviews were semi structured and contained 14 questions for the control care chains and 16 for the acute care chain for AAC. The 14 questions posed to both groups concerned factors possibly influencing implementation of the redesign and were based on implementation literature. More explicit, participants were posed questions about organizational routines ${ }^{18-}$ ${ }^{20}$, organizational factors such as care chain coordination and policy of participating institutions $^{21-23}$, a sense of urgency for chang $\mathrm{e}^{21-23}$ and the implementation methods used. The AAC care chain was posed two extra questions concerning the use of the redesign after implementation and the implementation techniques used. In the control care chains, the moderator presented the redesign as developed for the acute care chain for AAC and asked the respondents to answer as if this redesign were to be implemented into the care chain in which they were involved.

\section{Data analysis}

The responses to the questionnaires were entered into one database for all six acute care chains using SPSS 16.0. Frequency tables were produced for all characteristics of the acute care chains and for each characteristic, a direction of the influence was given (i.e. negative or positive). Missing values were entered into the database as unknown. The FG interviews were recorded and transcribed verbatim to increase validity. Answers were entered into data matrices ${ }^{17}$, one for the acute care chain of ACC and one for the control care chains. The rows of the data matrices contained the factors possibly influencing implementation. The 'bracketing' technique ${ }^{24}$ was used to fill the cells, firstly containing so called thick descriptions (i.e. literal interview passages). Thick descriptions were coded by one researcher - based on the pre-defined concepts - and then transformed into 'thin' descriptions - i.e. summarizing the respondents answers per concept. The data matrices containing thick and thin descriptions were discussed by two researchers (JVLZ and AVR) until consensus about the content of the data matrices was reached.

Using two data collection methods allowed us to cross examine the data (data triangulation), increasing validity and reliability as well as objectivity and credibility of the study findings. The RATS guideline was followed to ensure quality of reporting of the study ${ }^{25}$.

\section{Ethical Considerations}

The study was carried out in accordance with the standards of expected ethical behavior based on The Code of Ethics of the World Medical Association ${ }^{26}$. According to national regulation, full ethical approval was deemed unnecessary because participants in this study were not 
subject to any acts, nor were they forced to change their behavior at any point during the study ${ }^{27}$. Anonymity was guaranteed to both the respondents of the questionnaires and the FG participants.

\section{RESULTS}

Seven FG interviews were planned with five eventually being held (Table 1). For AOC, no coordinating group existed yet and we were unable to set a date with a representative group of care providers in the field to replace the coordinating group. The coordinating group for AMI was splitting up into two groups at the time of the study and it was therefore not possible to plan a FG interview for this care chain. The length of the FG interviews varied from thirty minutes to one and a half hours, depending on the time available. Time constraints meant not all questions were answered.

Table 1 - Overview of Focus Group interviews

\begin{tabular}{lll}
\hline Acute care chain & Participants & Non response \\
\hline CVA & 7 & \\
Myocardial infarction & - & Split in care chain coordination, FG interview not feasible \\
Acute Obstetrics & - & no existing FG \\
Acute hip traumas & 11 & \\
Acute psychiatrics & 14 & \\
Acute abdominal complaints & 4 & \\
Implementation group & 3 &
\end{tabular}

Questionnaires were sent to 40 care providers and 23 were returned (response rate $57.5 \%$ ). Response rates varied between acute care chains, varying from $42.8 \%$ (CVA) to $80 \%$ (APC) (Table 2). Results were categorized according to routines, organization, sense of urgency and implementation methods. Besides barriers to implementation being explored, facilitators to implementation were also discussed by respondents.

Table 2 - Overview of Response to Questionnaires

\begin{tabular}{|c|c|c|c|}
\hline Acute care chain & Questionnaires send & Questionnaires returned & $\%$ \\
\hline CVA & 7 & 3 & 42.8 \\
\hline Myocardial infarction & 7 & 5 & 71.4 \\
\hline Acute Obstetrics & 8 & 5 & 62.5 \\
\hline Acute hip traumas & 6 & 3 & 50.0 \\
\hline Acute psychiatrics & 5 & 4 & 80.0 \\
\hline Acute abdominal complaints & 7 & 3 & 42.9 \\
\hline Total & 40 & 23 & 57.5 \\
\hline
\end{tabular}




\section{Routines}

Respondents indicated that existing routines in the organization might have acted as barriers for implementation in several ways. Firstly, respondents mentioned that routines differed between the organizations involved in the care chain. Respondents in the acute care chain for AAC mentioned that

"giving feedback is more common amongst specialists than it is amongst GPs, because of peer reviews and handovers at shift change".

Respondents from the acute care chain for AHT answered "yes", when asked to indicate whether work routines in general differ amongst individual providers involved in the acute care chain. In the FG with the acute care chain for CVA, respondents indicated that

"everyone has their own perspective" and "on top of that, all hospitals work differently"

Secondly, respondents mentioned that the redesign differed from the current routines of organizations involved the care chain in that

"the feedback form is not digital yet" (AAC)

Respondents from the acute care chain for AHT mentioned that

"... we have never done this before so we don't have a routine. Like we said, we mostly work digitally, so a paper form doesn't actually fit."

Participants in the acute care chain for CVA mentioned that

"we want to start a certain treatment as soon as possible, so anything that adds logistic throughput or paperwork to the process is a problem"

indicating that the redesign adds work to the existing routines. Finally, participants mentioned that

"it [providing feedback] is not an explicit role between GP, specialist and ambulance caregiver..." and that "it is not a habit - apart from the question of whether professionals find it useful - it is just an extra task" (AAC)

In the FG with the acute care chain for AHT, respondents mentioned that

"For some it's a habit, but for most it's not a habit to do this [providing feedback]"

In the acute care chain for APC, respondents noted that

"in acute psychiatric care, we always say, 'don't just provide feedback on paper, but also call" indicating that the redesign does not match with the routine. Results from the questionnaires demonstrate that $21.7 \%$ (information transfer) to $30.4 \%$ (feedback) of the respondents believe that routines existing information transfer routines negatively influence implementation. Only $13 \%$ of the respondents believe that existing information transfer routines and feedback positively influence implementation. 
Table 3 - Barriers and Facilitators Mentioned in Focus Groups

\begin{tabular}{|c|c|c|}
\hline Acute care chain & Barrier & Facilitator \\
\hline $\begin{array}{l}\text { Implementation } \\
\text { care chain } \\
\text { (acute abdominal } \\
\text { complaints) }\end{array}$ & 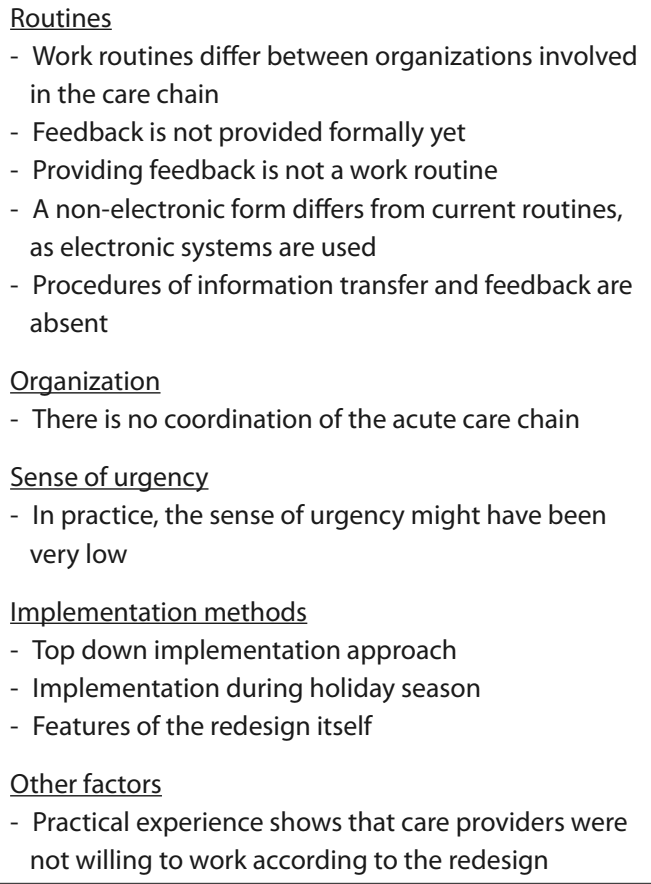 & $\begin{array}{l}\text { Routines } \\
\text { - } \\
\text { Organization } \\
\text { - The redesign should fit } \\
\text { into the organization's } \\
\text { policy } \\
\text { Sense of urgency } \\
\text { - On a higher organiza- } \\
\text { tional level, there was } \\
\text { a sense of urgency for } \\
\text { improvement } \\
\text { Other factors } \\
\text { - The redesign is desirable }\end{array}$ \\
\hline $\begin{array}{l}\text { Control care } \\
\text { chains* } \\
\text { (acute hip } \\
\text { traumas, acute } \\
\text { psychiatric care, } \\
\text { CVA) }\end{array}$ & $\begin{array}{l}\text { Routines } \\
\text { - Work routines differ between districts and organiza- } \\
\text { tions involved in the care chain } \\
\text { - The redesign differs from the current work routines } \\
\text { - Organizations are used to work with digital systems } \\
\text { instead of paperwork. } \\
\text { Organization } \\
\text { - There is no coordination of the acute care chain } \\
\text { Sense of urgency } \\
\text { - A need for improvement in information transfer and } \\
\text { feedback is experienced, although may not be very } \\
\text { urgent } \\
\text { Implementation methods } \\
\text { - Top down implementation approach } \\
\text { - Implementation during holiday season } \\
\text { - Features of the redesign itself } \\
\text { Other factors } \\
\text { - Willingness to work with the redesign depends } \\
\text { on whether it is digital or not. As a paper version, } \\
\text { willingness would not be very high }\end{array}$ & $\begin{array}{l}\text { Routines } \\
\text { - } \\
\text { Organization } \\
\text { - The redesign should fit } \\
\text { into the organization's } \\
\text { policy } \\
\text { Sense of urgency } \\
\text { - } \\
\text { Other factors } \\
\text { - The ideas behind the } \\
\text { redesign are probably } \\
\text { desirable }\end{array}$ \\
\hline
\end{tabular}

*Acute care chains for Obstetrics and myocardial infarction are missing here since no FG interview was held with those care chains. 


\section{Organizational aspects}

On an organizational level, the absence of a coordinator for the care chains was mentioned as a possible barrier to implementation. Respondents mentioned that

"there is no such thing as a hierarchy in which a protocol can be established, it should be based on equality" (AAC)

Additionally, the absence of a coordinator

"doesn't have to be a barrier, but in this case it probably was" (AAC)

Respondents in the acute care chain for AHT confirmed the negative influence of the absence of a coordinator with a simple "yes" and indicated that

"it does have an adverse effect; nobody tells you what to do"

The respondents in the acute care chain for CVA also confirmed that

"we don't have a care chain coordinator"

The division of responsibilities and authority between providers in the care chain is believed to negatively influence implementation according to $30 \%$ of respondents of the questionnaires. Furthermore, inadequate cooperation between providers in the care chain $(26.1 \%)$ and the nonexistence of a protocol for information transfer on the care chain level (26.1\%) is mentioned as negatively influencing implementation (Table 4). Besides barriers, respondents mentioned a facilitator on this topic. The redesign should fit with the organizational policy according to respondents, thus stimulating implementation. When asked whether the redesign does not fit with organizational policy, respondents indicated

"on the contrary, it is really good to do it according to protocol [information transfer and feedback]" (CVA)

Participants state that

"you should not ask whether it fits [into the organizational policy], because it should. It is a quality improvement impulse and you do want the quality of the care chain to improve" (AHT)

Respondents in the acute care chain for AAC concurred,

"we all have a quality system in which you think that way"

In addition to matching the redesign with organizational policy, cooperation between multiple disciplines and organizations was identified as having a fairly positive influence on implementation by $39.1 \%$ and $34.8 \%$ of respondents respectively (Table 3 ).

\section{Sense of urgency}

Respondents from all care chains indicated that a sense of urgency for the improvement of information transfer and feedback existed, although this might not have been perceived as very urgent. Participants mentioned that 
"it was the main conclusion from the ROAZ [Dutch acronym for: Regionaal Overleg Acute Zorg, in English: Regional Consultative body for Acute Care] meeting two years ago, that there is a lack of feedback. We don't know what happens with a patient so we don't have any learning points" (Implementation Group)

and that

"a sense of urgency did exist" (AAC)

Furthermore, respondents from the acute care chain for AHT mentioned that

"... regularly, there is a shortage of information for our patients"

and that the problem

"might not be very urgent, but it is very unpleasant of course"

When discussing the sense of urgency in the FG with the acute care chain for CVA, it is mentioned that

"reading between the lines, I believe we think that improvement is possible and we also think it is needed"

Finally, respondents stated that

"It [the current state of handover communication] can always be improved"

and

"it [handover communication, can be faster" (APC)

The need for improvement of information transfer and feedback is indicated to be fairly to very urgent by $39.1 \%$ (information transfer) and $43.4 \%$ (feedback) of respondents (Table 4).

\section{Implementation methods}

Possible barriers relating to implementation methods were discussed in the FG interviews with the acute care chain for AAC and with the implementation group (IG). Three barriers were mentioned.

Firstly, respondents stated that

"the approach was top down, that might not work with professionals" (IG) and "I think that we might question whether we sufficiently introduced the redesign at the professional level" (IG)

Secondly, the timing of the implementation is mentioned as a barrier;

"Maybe the implementation period influenced implementation, it was holiday season at that time" (AAC)

Finally, the features of the redesign itself - mainly concerning not using the electronic capabilities of an organization - were mentioned as a barrier towards implementation. Respondents mentioned that 
"above all, the form should be a digital one, it should be a part of your medical file... it is like that because we live in a digital age. If we were still using paper patient records, this form would have been a part of the record" (IG)

and

"if the form is digital, you don't have the chance to get it returned blank, you simply get a pop-up from the system and have to fill out the form before you can proceed"(AAC)

The results of the FG interviews and questionnaires mostly correspond with each other, with the exception of the sense of urgency feature. Respondents from the FG interviews mention this feature as a barrier to implementation, whereas respondents from the questionnaire indicate to experience a fairly to very high sense of urgency.

An overview of the barriers and facilitators to implementation derived from the FG interviews and questionnaire is shown in tables 3 and 4.

\section{DISCUSSION}

In this study, perceived barriers to implementation of a redesign for information transfer and feedback in acute care chains were defined. Based on the responses of the healthcare professionals, these barriers can be grouped into three main categories, relating to: (a) existing routines, (b) implementation method and (c) a low sense of urgency for improvement. As implementation strategies tailored to specific barriers to change seem to be more effective as general strategies ${ }^{22}$, the identification of these barriers provide valuable insight for acute care practice and the field of implementation science.

\section{The role of existing routines in implementation processes}

Most barriers to implementation of the redesign mentioned by respondents related to routines, indicating that changing existing routines might play an important role in successful implementation of the redesign. Organizational routines are often described as having a high level of stability, leading to organizational inertia ${ }^{20}$. At the same time, organizational routines are also described as sources of continuous change, as repetition of the same routine by multiple actors leads to a variety of performances ${ }^{20}$. Recent research shows the importance of memory in changing organizational routines ${ }^{18}$. Whereas transactional memory enhances adaptation to changes in organizational routines, declarative memory - building from past experiences - can act as a barrier ${ }^{18}$. Changing an organizational routine is therefore not only a matter of learning a new routine, it also involves unlearning the old ${ }^{18,28}$. Feedback has shown to be an efficient method in learning new (communication) routines ${ }^{29,30}$ and was therefore an important component of the redesign for information transfer and feedback. In line with recent research ${ }^{31}$, results show however that providers are largely unknown with providing feedback to each other. Providing only an opportunity for feedback on existing routines was not sufficient for changing them. Literature on unlearning suggests that openness to vulnerability, willingness to listen, reflection of feeling and a high tolerance for raised feelings are important qualities for unlearning ${ }^{32}$. 


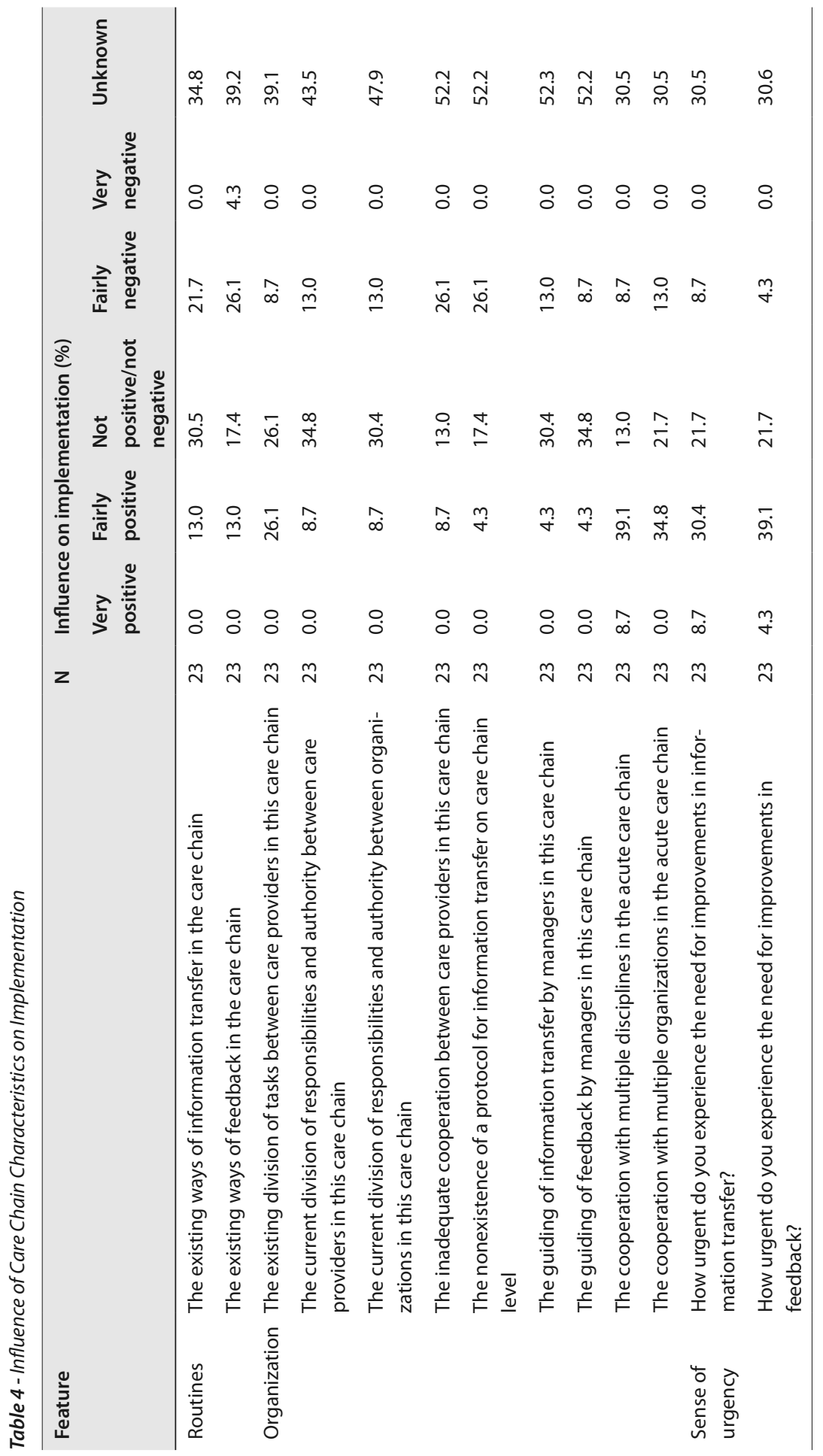


Hence, organizations should focus on creating an environment supporting creativity, vulnerability and openness to stimulate unlearning ${ }^{32}$. In addition, respondents mentioned that the redesign deviated too much from the existing routine. More specific, respondents mentioned that the redesign not being integrated into the electronic systems of the organizations was one of the barriers to implementation. Not only because it introduced extra work, but also because the redesign now deviated from the existing routines. These are important insights, as originally feedback was implemented as a tool aiming to change the existing routine. In practice, the actors in the acute care chain felt confronted with a new routine, whilst also having to change the existing routine. If however, the redesign would have been introduced electronically - as a 'manual' variation of an existing routine, i.e. the electronic patient record - chances of successful implementation might have been higher. Not only would adaptation to this new routine probably be easier since it stores new learning (using feedback forms) along with the old (using the electronic patient record) ${ }^{33,34}$, it also would have been possible to be stricter on the performance of the new routine, by obligating professionals to use the form before they could continue to work in the patient record.

\section{The role of implementation methods}

The top down approach to implementation was indicated as a barrier in this study. This conclusion helps explain why implementation strategies might be more effective if tailored to the different levels within an organization. Hence, this conclusion might also be related to the previous point of changing organizational routines. Seeing routines as sources of continuous change leads to the understanding that change of an organizational routine originates from endogenous factors ${ }^{20}$. Choosing a top down implementation approach however, is based on providing an external stimulus, rather than searching for endogenous factors that would facilitate the same goal.

\section{The importance of a high sense of urgency}

Although respondents indicated a sense of urgency to be present, the level experienced was generally low. A high sense of urgency amongst the users of an intervention is an important factor in successful implementation ${ }^{23}$. Participants should prioritize the implementation process, which should be addressed in implementation projects ${ }^{23}$. Responses from the questionnaires indicate a fairly to very urgent need for improvement. In this area the lack of an overall high sense of urgency was not enough and acted as a barrier to implementation. In addition, establishing a high sense of urgency amongst all healthcare providers involved might be key to successful implementation here ${ }^{35,36}$. From the results of this study, it can be doubted that throughout the acute care chains, the same sense of urgency was present. Communicating with involved healthcare providers about the problems and possibilities of the redesign could already help to establish a sense of urgency. 


\section{Study limitations}

The context of this study and the methods used in this study produce some limitations. First, routines are embedded in organizational rules and can differ from one organization to another, highlighting the importance of organizational features. Additionally, context-specific data collection might be perceived as a limitation. However, as a specific context is the object of study, this is inherent to case study research ${ }^{37}$. Nevertheless, case study research is important to develop a nuanced understanding of the real-life context in which theories are applied ${ }^{38}$ as was the specific aim of this study. The number of respondents to the questionnaires $(n=23)$ can be noted as a limitation to this study, however, purposive sampling and cross examination of data was used to address this problem. A relatively large number of respondents answered questions with "don't know" - ranging from $26.1 \%$ to $52.2 \%$. This could be explained by either a fault in the purposive sampling or by the nature of the questions posed. The use of FG interviews for data collection may also be a limitation of the study. It might be possible that we missed some barriers due to the content and structure of the semi structured FG interviews. Some literature suggests the role of the moderator during the FG interviews might disturb the data collection process; i.e. since the moderator determines the agenda of the FG interview, answers are more or less restricted to this agenda ${ }^{39}$. In this study however, although the agenda for the FG interviews was set by the moderator, the moderator let the participants freely elaborate on each topic. The moderator was well trained and only moved on to the next topic when data saturation was reached. Apart from the influence of the moderator, FG interviews are considered to be an appropriate data collection tool when examining complex behaviours and motivations, as was done in this study ${ }^{39}$. The difference in group size between the FGs may have resulted into different group interactions, but the effect this has on the results of the FG interviews is considered to be small ${ }^{39}$. Coding of thick descriptions into thin description by only one researcher might influence validity of the findings, although we used pre-defined concepts for coding to address this problem. Within the implementation literature, numerous factors are known to influence implementation of healthcare innovations ${ }^{21,22,40}$ and not all of these were specifically addressed in this study as we were interested in those barriers perceived by healthcare professionals. The findings of this study therefore do not reflect all barriers to implementation. The outcomes remain important, as the barriers mentioned are perceived by the respondents to have been of significant influence in the selected cases of acute care chains.

\section{CONCLUSIONS}

In general, these study results show a number of perceived barriers towards implementation. Most of these barriers were related to organizational routines. The study results underline the importance of understanding that, when implementing a redesign of a process, implementation strategies should be tailored to the different actors involved. Additionally, a high sense of urgency might be an important prerequisite for implementation. Future implementation efforts should therefore start off with the establishment of a high sense of urgency amongst involved care providers. Most importantly, in implementation efforts unlearning the existing 
routine should receive equal attention as learning to use a new routine. Further research is needed to understand which specific routines and contexts are important to address here and how routines are to be unlearned. Hence, the likelihood of success in future efforts to improve communication in acute care will increase as specific attention is paid to unlearning existing communication routines.

\section{COMPETING INTERESTS}

The authors declare that they have no competing interest.

\section{AUTHORS' CONTRIBUTIONS}

JVLZ undertook the data collection, analysis and drafted the manuscript. AVR was study lead and helped draft the manuscript. IDP was a member of the project group carrying out the study and helped draft the manuscript. MK contributed to study development and helped draft the manuscript. DR helped draft the manuscript. PB was member of the project group, leader of the implementation group and helped draft the manuscript. HV contributed to study development and helped draft the manuscript. All authors read and approved the final manuscript.

\section{ACKNOWLEDGEMENTS}

This article is based on data collected for the research project "Improvement of communication and information transfer in acute care chains by implementing a model for information transfer and feedback"(in Dutch: "Verbetering van communicatie en informatieoverdracht in ketens voor spoedzorg door invoering van een model voor informatieoverdracht en feedback") This research project is carried out by means of a grant from ZonMw. The authors would like to thank ZonMw for funding the project and the projectgroup which consists of members from Maastricht University, the $\mathrm{MUMC}^{+}$, the Huisartsenpost Maastricht Heuvelland, the GGD ZuidLimburg, the Traumacentrum Limburg and the Regionale Huisartsenzorg Heuvelland. The authors would also like to thank all respondents for participating in this research project. Finally, we would like to thank Hayley Brown for checking the manuscript for the correct use of the English language. 


\section{REFERENCES}

1. Cheung DS, Kelly JJ, Beach C, et al. Improving handoffs in the emergency department. Ann Emerg Med. 2010;55(2):171-180.

2. Donaldson MS. Continuity of care: a reconceptualization. Med Care Res Rev. 2001;58(3):255-290.

3. Haggerty JL, Reid RJ, Freeman GK, Starfield BH, Adair CE, McKendry R. Continuity of care: a multidisciplinary review. BMJ. 2003;327(7425):1219-1221.

4. Manser T, Foster S. Effective handover communication: an overview of research and improvement efforts. Best Pract Res Clin Anaesthesiol. 2011;25(2):181-191.

5. Beach C, Croskerry P, Shapiro M. Profiles in patient safety: emergency care transitions. Acad Emerg Med. 2003;10(4):364-367.

6. Sandkuhl K, Filipe J, Cordeiro J, Cardoso J. Information Logistics in Networked Organizations: Selected Concepts and Applications Enterprise Information Systems. In: Aalst W, Mylopoulos J, Rosemann M, Shaw MJ, Szyperski C, eds. Vol 12: Springer Berlin Heidelberg; 2009:43-54.

7. Patterson PD, Pfeiffer AJ, Weaver MD, et al. Network analysis of team communication in a busy emergency department. BMC Health Serv Res. 2013;13:109.

8. Creswick N, Westbrook J, Braithwaite J. Understanding communication networks in the emergency department. BMC Health Serv Res. 2009;9:247.

9. Traumacentrum Limburg. Failure Mode and Effects Analysis CVA, Myocardinfarct, Heuptrauma, Obstetrie, Psychiatrie. Maastricht: Traumacentrum Limburg; 2009.

10. Chalmers I, Glasziou P, Godlee F. All trials must be registered and the results published. Brit Med J. 2013;346.

11. Jamtvedt G, Young JM, Kristoffersen DT, O'Brien MA, Oxman AD. Audit and feedback: effects on professional practice and health care outcomes. Cochrane Database Syst Rev. 2006(2):CD000259.

12. Sargeant J, Curran V, Allen M, Jarvis-Selinger S, Ho K. Facilitating interpersonal interaction and learning online: linking theory and practice. J Contin Educ Health Prof. 2006;26(2):128-136.

13. Sargeant J, Mann K, Ferrier S. Exploring family physicians' reactions to multisource feedback: perceptions of credibility and usefulness. Med Educ. 2005;39(5):497-504.

14. Sargeant J, Mann K, Sinclair D, et al. Learning in practice: experiences and perceptions of highscoring a. Acad Med. 2006;81(7):655-660.

15. Huisartsenpost Maastricht Heuvelland. Huisartsenpost Maastricht Heuvelland: Organisatie. 2009; http://www.hapmaastricht.nl/organisatie.html. Accessed 14-04-2011, 2011.

16. van Uden CJ, Giesen PH, Metsemakers JF, Grol RP. Development of out-of-hours primary care by general practitioners (GPs) in The Netherlands: from small-call rotations to large-scale GP cooperatives. Fam Med. 2006;38(8):565-569.

17. Miles M, Huberman M. Qualitative data analysis: An expanded sourcebook (2nd ed.). Thousand Oaks, CA: Sage; 1994. 
18. Miller KD, Pentland BT, Choi S. Dynamics of Performing and Remembering Organizational Routines. J Manage Stud. 2012;49(8):1536-1558.

19. Novak L, Brooks J, Gadd C, Anders S, Lorenzi N. Mediating the intersections of organizational routines during the introduction of a health IT system. Eur J Inform Syst. 2012;21(5):552-569.

20. Pentland BT, Hrem T, Hillison D. The (N)Ever-Changing World: Stability and Change in Organizational Routines. Organ Sci. 2011;22(6):1369-1383.

21. Grol R. Successes and failures in the implementation of evidence-based guidelines for clinical practice. Med Care. 2001;39(8 Suppl 2):I146-54.

22. Grol R, Grimshaw J. From best evidence to best practice: effective implementation of change in patients' care. Lancet. 2003;362(9391):1225-1230.

23. Grol RP, Bosch MC, Hulscher ME, Eccles MP, Wensing M. Planning and studying improvement in patient care: the use of theoretical perspectives. Milbank Q. 2007;85(1):93-138.

24. Denzin NK. Interpretive Interactionism. Newbury Park, CA: Sage Publications; 1989.

25. Clark J. How to peer review a qualitative manuscript. In: Godlee F JT, ed. Peer Review in Health Sciences. 2nd edition. London: BMJ Books; 2003:219-235.

26. Association WM. Declaration of Helsinki, Ethical Principals for Medical Research Involving Human Subjects. 2008; http://www.wma.net/en/30publications/10policies/b3/index.html. Accessed 16-10-2013, 2013.

27. Ministerie van Volksgezondheid, Welzijn en Sport. Wet medisch-wetenschappelijk onderzoek met mensen. Vol BWBR00094082014. Den Haag: 2014

28. Akgun AE, Byrne JC, Lynn GS, Keskin H. Organizational unlearning as changes in beliefs and routines in organizations. J Organ Change Manag. 2007;20(6):794-812.

29. Smith S, Hanson JL, Tewksbury LR, et al. Teaching patient communication skills to medical students: a review of randomized controlled trials. Eval Health Prof. 2007;30(1):3-21.

30. Hovlid E, Bukve O, Haug K, Aslaksen AB, von Plessen C. Sustainability of healthcare improvement: what can we learn from learning theory? BMC Health Serv Res. 2012;12:235.

31. Hesselink G, Vernooij-Dassen M, Pijnenborg L, et al. Organizational culture: an important context for addressing and improving hospital to community patient discharge. Med Care. 2013;51(1):90-98.

32. Rushmer R, Davies HT. Unlearning in health care. Qual Saf Health Care. 2004;13 Suppl 2:ii10-15.

33. Bouton ME. Context, ambiguity, and unlearning: sources of relapse after behavioral extinction. Biol Psychiatry. 15 2002;52(10):976-986.

34. Edmondson AC, Bohmer RM, Pisano GP. Disrupted routines: Team learning and new technology implementation in hospitals. Admin Sci Quart. 2001;46(4):685-716.

35. Kotter JP. Leading Change - Why Transformation Efforts Fail. Harvard Bus Rev. 1995;73(2):59-67.

36. Kotter JP. A Sense of Urgency. Boston, Massachusetts: Harvard Business Press; 2008.

37. Yin RK. Case Study Research: Design and Methods Vol 5. 4 ed. Thousand Oaks: SAGE Inc.; 2009. 
38. Flyvbjerg B. Five Misunderstandings about Case-Study Research. Qualitative Inquiry. 2006;12(2):219-245.

39. Morgen DL. Focus Groups. Annual Review of Sociology. 1996;22:129-152.

40. Chaudoir SR, Dugan AG, Barr CHI. Measuring factors affecting implementation of health innovations: a systematic review of structural, organizationals, provider, patient and innovation level measures. Implementation Science. 2013;8(22). 



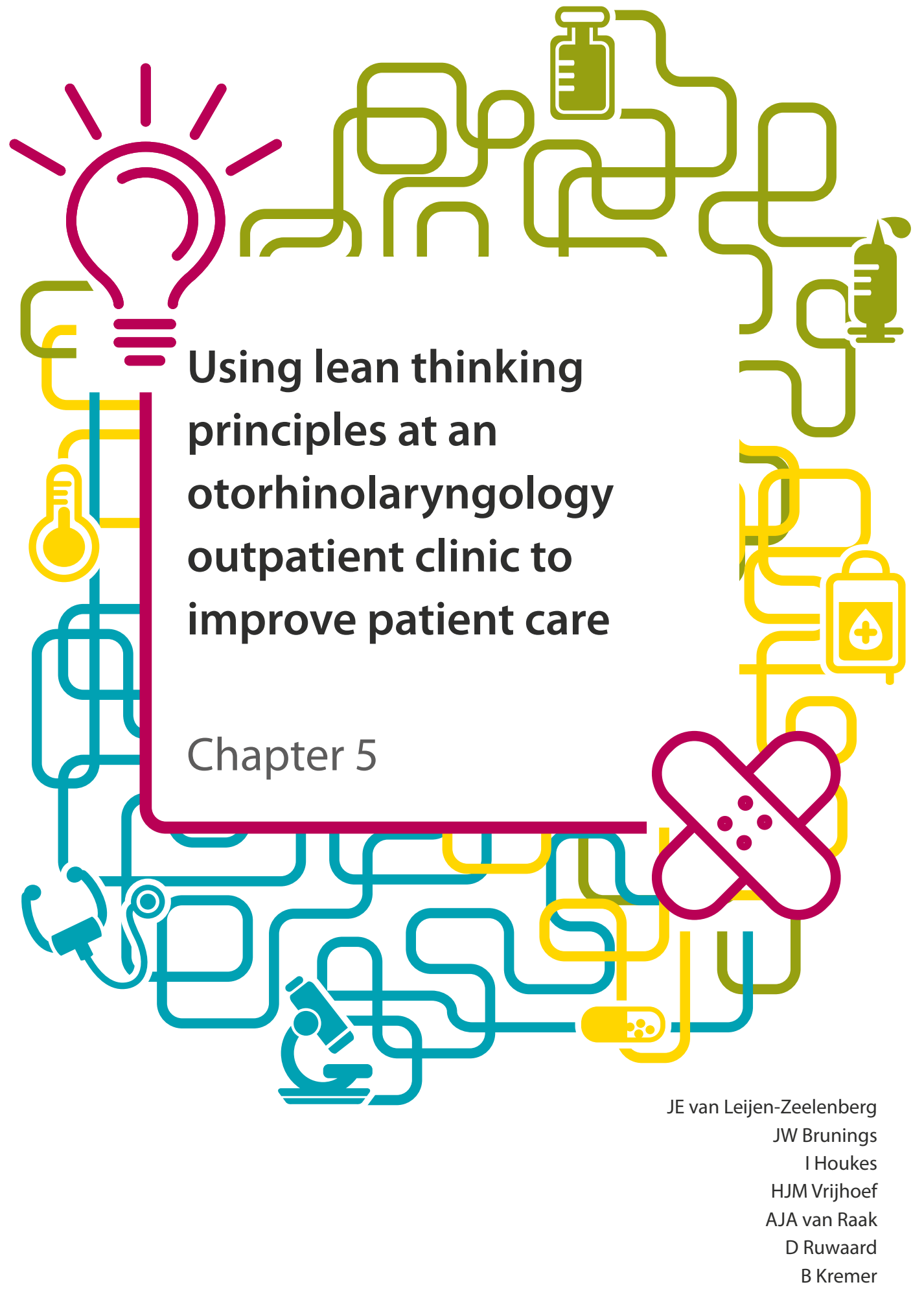

The Laringoscope, 2015; accepted for publication 


\section{Chapter 5}

\section{ABSTRACT}

\section{Introduction:}

Although Lean Thinking has led to considerable improvement in a variety of healthcare settings, its effects on otorhinolaryngology remain underexposed. This study reports on how the implementation of Lean Thinking at an otorhinolaryngology outpatient clinic has affected patient and provider satisfaction, waste reduction and organizational culture.

\section{Methods:}

The 18-month prospective before-and-after design used mixed methods for data collection and analysis. A survey was held to measure satisfaction among patients and providers. Semistructured interviews were conducted to evaluate the effect of Lean Thinking on waste and organizational culture.

\section{Main results:}

During the project, 69 issues were posted on the Lean board. Improvements were made on 36 inefficiency issues, not all concerning a specific type of waste. Employees reported considerable improvement in transportation, motion and waiting. Patient satisfaction was high both at baseline and follow-up and did not change significantly. The effects on provider satisfaction were slight; satisfaction with autonomy and participation decreased significantly but satisfaction with communication increased significantly.

\section{Conclusions:}

The implementation of Lean Thinking at an otorhinolaryngology outpatient clinic reduced waste and increased provider satisfaction with communication. Although patient satisfaction did not change significantly, it cannot be concluded that the intervention had no effect on perceived quality of care. Other approaches to measure patients' perceptions should be considered.

\section{Key words:}

Quality improvement, otolaryngology, outpatient clinic, Lean Thinking, mixed methods

\section{Level of Evidence:}

N/A (basic research) 


\section{INTRODUCTION}

Healthcare services must be redesigned to keep them sustainable and to address limitations in the quality of care as currently delivered ${ }^{1-5}$. Lean Thinking is a method to improve service delivery through redesign. Originating in the automotive industry, it is increasingly applied to healthcare ${ }^{6-8}$. Lean creates value in a system while eliminating waste ${ }^{6-9}$. Activities that do not add value to the customer are labeled 'waste'; in healthcare, these fall into seven types (Table 1),10. Full application of Lean should create flow (smooth and seamless processes) in a pull system (a service is only delivered when the customer asks for it). Lean organizations will continuously strive for perfection, implying continuous quality improvement.

Table 1 - Types of waste and application to Healthcare ${ }^{9,10}$

\begin{tabular}{ll}
\hline Type of waste & Application in Healthcare \\
\hline Transportation & Caused by poor layout: unnecessary movement of patients and staff \\
Inventory & Excess stock: e.g. not being used, patients waiting \\
Motion & $\begin{array}{l}\text { Motion not necessary for completion of the service: staff searching for equipment or } \\
\text { paperwork }\end{array}$ \\
Waiting & $\begin{array}{l}\text { Ile time between operations: waiting for results, staff or medicines, waiting caused } \\
\text { by schedule mistakes }\end{array}$ \\
Overproduction & $\begin{array}{l}\text { Production of components not for immediate use: unnecessary diagnostics, keeping } \\
\text { of time-slots } \\
\text { Doing more than necessary: unnecessary repeating of tests, asking for patient' } \\
\text { details several times } \\
\text { Steps that need correction: repeating tests because of errors, readmissions because } \\
\text { of failed discharge. }\end{array}$ \\
\hline
\end{tabular}

Healthcare settings as diverse as outpatient clinics, patient wards or surgical units are amenable to Lean ${ }^{11}$. As a recent literature review shows, its application to operating rooms, emergency departments or patient wards generally improves performance ${ }^{12}$. The implementation of Lean has increased patient satisfaction while enhancing staff morale by making more efficient use of their expertise ${ }^{13}$. So far, few studies have evaluated the effect of Lean on otorhinolaryngology (ORL) outpatient clinics ${ }^{14,15}$. Nor is much known about its impact on patient satisfaction, physician satisfaction, willingness to change or perceived openness ${ }^{14,15,16}$.

The objective of this study was to evaluate improvements made by implementing Lean and determine the effects on patient satisfaction, employee satisfaction, the amount of waste and organizational culture. 


\section{METHODS}

An 18-month prospective study with a before-and-after design using mixed methods for data collection evaluated the effects of Lean at an outpatient clinic. The project spanned a four-month baseline period (September 2011 - December 2011) leading into an 11-month redesign/ implementation period (January 2012 - December 2012) and a three-month follow-up period (December 2012 - March 2013).

\section{Setting}

Lean was implemented in a tertiary outpatient ORL clinic with an academic and regional function. In 2012, 20,534 patients were seen there. The ORL outpatient department is supported by an Audiology Center (AC) and a Vestibular Center (VC). Altogether, 63 employees are directly involved in patient care (including the $\mathrm{AC}$ and the $\mathrm{VC})^{17}$.

\section{Intervention}

Members of a project group were trained in the basics of Lean Six Sigma and received a 'yellow belt ${ }^{18}$. Two meetings were held with a representative group of the departmental staff to set forth the project aims, explain Lean Thinking and create a value stream map. Root cause analysis entailed asking "why" five times when waste was detected. Employees noted any waste on a 'Lean board' in the central conference room. At a monthly 'Lean session', all postings were discussed: (a) for existing points, progress was charted (status was green once the problem was solved, orange when being worked on, red if on hold); (b) for new points, problems were analyzed and employees assigned to work on them. Sessions were restricted to 30 minutes to avoid creating waste.

\section{Data collection}

Semi-structured interviews, a patient satisfaction survey and an employee satisfaction survey were used to collect data before and after the intervention. To ensure information-rich cases, respondents were selected through purposive sampling ${ }^{19}$. Employees directly involved in primary care were divided into groups (e.g., attending physicians, residents, interns, support staff, AC and VC staff). The project group decided which employees could participate. Attending physicians were all included because of their own special medical expertise. Four broad topics were raised during the interviews: (a) process characteristics; (b) waste; (c) strengths, weaknesses and changeability; and (d) cultural aspects. Beforehand, respondents were asked to give their consent for conducting the interview and recording it.

A patient satisfaction survey was administered before and after the intervention. The 'Core questionnaire for the assessment of Patient Satisfaction in academic hospitals' (COPS), which is commonly used in the Netherlands, is valid and reliable ${ }^{20}$. It was adapted to the project context by removing and adding questions and adding dimensions ${ }^{20}$. The core questionnaire was expanded by adding two dimensions related to otorhinolaryngology, making a total of eight. The questionnaire used for AC patients contained six dimensions; 'medical care' and 'combined appointments' were not included. A 5-point Likert-type scale (from 1=unsatisfied to 5=very 
satisfied) was used. Both questionnaires rated overall satisfaction on a scale of 1-10. The psychometric characteristics of the amended questionnaires were tested and all dimensions showed good reliability $(a>0.7)$. When checking the item discriminant validity, all items showed the strongest correlation to the dimension in which they belonged.

Employee satisfaction was measured pre and post intervention. The questionnaire contained nine dimensions and included an overall satisfaction rate. Eight dimensions (job content, organization of work, work environment, mental and emotional burden, social support, participation, intrinsic work motivation, commitment) were retrieved from a questionnaire that had been validated previously ${ }^{21,22}$ and one dimension (ergonomics) is comprised of two scales ${ }^{23,24}$. All scales, with the exception of work environment and ergonomics, had a good reliability score (a>0.7).

\section{Data analysis}

Semi-structured interviews were audio-recorded and transcribed verbatim to increase validity. All interviews were held in the Dutch language. The analysis was also carried out in Dutch, but the results were translated into English for reporting purposes. A template approach ${ }^{25}$ was used for the analysis, with the topics of the interview guide serving as the code book. NVivo $10^{26}$ was used to generate the final version, which consisted of 21 codes. Answers were entered into data matrices ${ }^{27}$, one for pre and one for post measurement. The rows contained the codes - the concepts used to phrase the questions. The 'bracketing' technique ${ }^{28}$ was used to fill the cells, initially with 'thick' descriptions (verbatim passages), which were then transformed into 'thin' descriptions by summarizing the answers per concept. A third data matrix was then made; the rows contained the codes while the columns contained pre-measurement, post-measurement and compared values.

Both satisfaction surveys were analyzed with SPSS 21 . Since data on patient satisfaction was collected from two groups, the differences between the groups were calculated with a $X^{2}$ test. Differences between pre- and post-measurement values were calculated with an independent samples t-test. Significance was measured one-sided, since we hypothesized that patients would be more satisfied after the intervention. The employee satisfaction survey was handed out to the same respondents before and after the intervention. Consequently, a dependent samples t-test was used for all scales to calculate differences between pre and post measurement. Here too, significance was measured one-sided, having hypothesized that employees would be more satisfied afterwards.

Using multiple data sources allowed us to cross-examine the data, thereby increasing the validity and reliability as well as the objectivity and credibility of the findings.

The study was carried out in accordance with the Code of Ethics of the World Medical Association ${ }^{29}$. According to Dutch national regulations, full ethical approval was deemed unnecessary ${ }^{30}$. Respondents to the questionnaires and the interviews were guaranteed anonymity. The SQUIRE guidelines were used to increase the quality of reporting of this study ${ }^{31}$. 
Table 2 - Overview of issues reported on Lean board

\begin{tabular}{|c|c|c|c|c|}
\hline Type of waste & Status green & Status orange & Status red & Status white \\
\hline Transportation & 1 & 1 & 0 & 0 \\
\hline \multicolumn{5}{|c|}{ Example (status red) } \\
\hline \multicolumn{5}{|c|}{ Problem: More space at the reception desk is needed to avoid patients walking on and of } \\
\hline \multicolumn{5}{|c|}{ Solution: The reception area will be altered in the upcoming renovation of the outpatient clinic } \\
\hline Inventory & 1 & 2 & 0 & 0 \\
\hline
\end{tabular}

Example (status green)

Problem: Treatment room 6 is messy and always running out of inventory

Solution: Drawers are rearranged so that it is easy to oversee what equipment is missing
Motion
3
0
2
0
5

Example (status green)

Problem: We need to search for equipment at each consultation hour, due to missing inventory and varying inventory in rooms)

Solution: A list with standard equipment and amounts for each room is made, rooms are refilled 2 or 3 times a day

Waiting

9

2

3

15

Example (status orange)

Problem: The audiology consultation hour is delayed in cases of ear wax removal

Solution: Explore problem and make improvement plan

Overproduction

3

0

0

4

Example (status green)

Problem: The monthly staff schedule is often late, resulting in rescheduling of patients

Solution: Creating a basic schedule that is not dependent on other departments

$\begin{array}{lllll}\text { Over-processing } & 4 & 2 & 3\end{array}$

10

Example (status red)

Problem: The patient needs to report at the reception desk multiple times if appointments are combined Solution: Develop different system for appointments
Defects
10
3
0
1

Example (status green)

Problem: Patients for the specialist hearing aid consultation hour are planned on the regular time spots Solution: Change the name of the consultation hour to stress the difference to both patients and employees

Other

8

4

0

4

16

Example (status green)

Problem: Not everybody is informed on project agreements

Solution: Inform employees by email after each meeting

Total

37

17

8

7

69 


\section{RESULTS}

\section{Lean improvement events}

Over the entire project, the Lean board accumulated 69 issues (including those identified at the outset). At the end of the project, 36 had a green status, 18 orange, nine red, and six remained to be coded (Table 2). Most issues concerned waiting (15), over-processing (12) and defects (10). Some (18) did not concern a specific type of waste but focused on problems like missing personal information about colleagues (Table 2).

\section{Waste}

Waste was identified during the interviews and by using the Lean board. At baseline, 31 employees were approached to participate in the study, and semi-structured interviews were held with 26 (response rate $83.8 \%$ ). At follow-up, 28 employees were approached to participate and semi-structured interviews were held with 23 respondents (response rate $82.1 \%$ ).

Differences in waste experienced by employees at baseline and follow-up concerned transportation, motion and waiting. Respondents indicated that although improvement had been made, these types of waste still occurred. In the words of a resident,

"What I think is still inefficient is that doctors have to search for the right equipment. Although I can say that things have already improved greatly, it still happens that I have to go and search for materials."

Most of the improvements seem to come from making basic equipment available during consultation hours. For inventory, a better arrangement was mentioned. As an attending physician explained,

"Yes, the drawer has been improved because indeed it is more structured now. First I couldn't find anything. But it sure has improved; if you open a drawer it is not a mess, but it is nicely organized."

A new referral system implemented throughout the hospital was experienced as having a negative influence on overproduction and over-processing .

"Well, we have this new system now, it is still a pilot [...] Well in this system the patient needs to be seen within three days and it happens that a patient who needs a combined appointment only gets one of those appointments because only one appointment is available and needs to be rescheduled afterwards, because he needed the combined appointment in the end." (administrative assistant)

Defects are present in the process, but respondents saw them as exceptional, both at baseline and follow-up. Only planning mistakes are common:

"Yes mistakes are made with planning patients, for instance planning patients at the wrong consultation hour. I think that mistake is the most common one." (audiologist)

The results of the interviews are summarized in Supplementary Table 1. 


\section{Patient satisfaction}

The patient satisfaction questionnaire was returned by 134 persons (response rate $89.3 \%$ ) at baseline and 76 (response rate 50.6\%) at follow-up. There were no statistically significant differences between the groups at baseline and follow-up regarding their characteristics (Table 3).

Table 3- Patient Characteristics - independent groups

\begin{tabular}{|c|c|c|c|c|}
\hline & & Baseline( $\mathrm{N}=134)$ & Follow-up ( $\mathrm{N}=76)$ & $X^{2}$ \\
\hline \multirow[t]{5}{*}{ Age } & $<20$ & $18(13.4 \%)$ & $10(13.2 \%)$ & 0.356 \\
\hline & $20-39$ & 24 (17.9\%) & $6(7.9 \%)$ & \\
\hline & $40-54$ & $25(18.7 \%)$ & $16(21.1 \%)$ & \\
\hline & $55-59$ & $14(10.4 \%)$ & $11(14.5 \%)$ & \\
\hline & $>60$ & $53(39.6 \%)$ & 33 (43.4\%) & \\
\hline \multirow[t]{2}{*}{ Sex } & Male & $63(47.0 \%)$ & $44(58.7 \%)$ & 0.106 \\
\hline & Female & 71 (53.0\%) & 31 (41.3\%) & \\
\hline \multirow[t]{2}{*}{ Native Language } & Dutch & 131 (98.5\%) & 76 (100\%) & 0.283 \\
\hline & Other & $2(1.5 \%)$ & $0(0 \%)$ & \\
\hline \multirow[t]{3}{*}{ Education level } & Low & $27(23.3 \%)$ & $18(25.4 \%)$ & 0.487 \\
\hline & Medium & $50(43.1 \%)$ & 35 (49.3\%) & \\
\hline & High & 39 (20.9\%) & $18(25.4 \%)$ & \\
\hline
\end{tabular}

In general, patient satisfaction scores were already high at baseline. Except for information provided at the $A C$, no statistically significant differences after the intervention were found (Table 4). Patients were more satisfied $(M=4.20, S D=0.65)$ with the information provided at the $A C$ before the intervention than afterwards $(M=3.85, S D=0.91, t(78)=2.001, p=0.025, r=0.22)$, but the effect is small. 


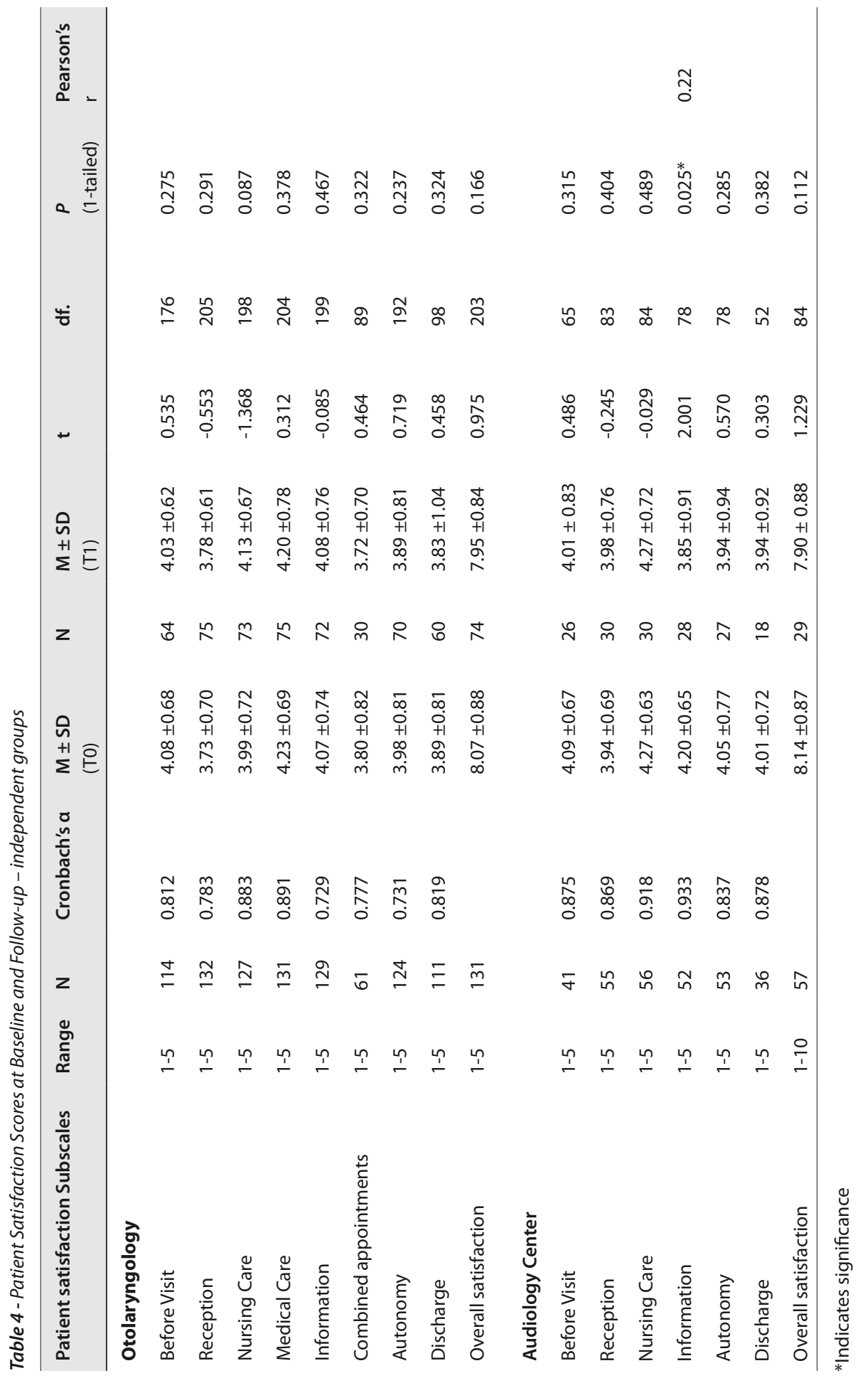




\section{Employee satisfaction}

At baseline, 49 out of 63 employees (77.8\%) returned the questionnaire; at follow-up, 36 of the $63(57.1 \%)$ returned it. Out of the 49 employees who took part at baseline, 21 did not participate at follow-up and were therefore excluded from the analysis. Although reasons for not participating were not systematically gathered, some of the drop-out may be attributed to natural outflow and external internships of residents. There is no significant difference between the drop-outs and the cohort (Table 5).

Table 5 - Employee Characteristics

\begin{tabular}{|c|c|c|c|c|}
\hline & & Cohort Data $(\mathrm{N}=28)$ & Dropout $(\mathrm{N}=21)$ & $X^{2}$ \\
\hline \multirow[t]{3}{*}{ Age } & Mean (SD) & $38,8( \pm 9.9)$ & $37,1( \pm 10.9)$ & \multirow{3}{*}{$\begin{array}{l}0.466 \\
\text { (Mann- } \\
\text { Whitney) }\end{array}$} \\
\hline & Min & 25 & 25 & \\
\hline & Max & 61 & 60 & \\
\hline \multirow[t]{2}{*}{ Sex } & Male & $11(39.3 \%)$ & $6(28.6 \%)$ & \multirow[t]{2}{*}{0.436} \\
\hline & Female & $17(60.7 \%)$ & 15 (71.4\%) & \\
\hline \multirow[t]{6}{*}{ Education } & Elementary school & $0(0 \%)$ & $0(0 \%)$ & \multirow[t]{6}{*}{0.750} \\
\hline & $\begin{array}{l}\text { Lower vocational } \\
\text { education }\end{array}$ & $0(0 \%)$ & $1(5 \%)$ & \\
\hline & $\begin{array}{l}\text { Intermediate vocational } \\
\text { education }\end{array}$ & $4(14.8 \%)$ & $4(20 \%)$ & \\
\hline & $\begin{array}{l}\text { Higher Professional } \\
\text { education }\end{array}$ & $8(29.6 \%)$ & $6(30 \%)$ & \\
\hline & University & $14(51.9 \%)$ & $8(40 \%)$ & \\
\hline & Other & $1(3.7 \%)$ & $1(5 \%)$ & \\
\hline \multirow{2}{*}{$\begin{array}{l}\text { Management } \\
\text { position }\end{array}$} & Yes & $9(32.1 \%)$ & $3(14.3 \%)$ & \multirow[t]{2}{*}{0.150} \\
\hline & No & 19 (67.9\%) & 18 (85.7\%) & \\
\hline \multirow[t]{4}{*}{ Appointment } & Permanent & $22(78.6 \%)$ & $16(76.2 \%)$ & \multirow[t]{4}{*}{0.864} \\
\hline & Temporary & $6(21.4 \%)$ & $5(23.8 \%)$ & \\
\hline & Fulltime & 18 (64.3\%) & 13 (61.9\%) & \\
\hline & Part-time & $10(35.7 \%)$ & $8(38.1 \%)$ & \\
\hline \multirow{3}{*}{$\begin{array}{l}\text { Satisfaction } \\
\text { grade }\end{array}$} & Mean (SD) & $7.87( \pm 0.6)$ & $7.95( \pm 0.6)$ & \multirow[t]{3}{*}{0.781} \\
\hline & Min & 7 & 7 & \\
\hline & Max & 9 & 9 & \\
\hline
\end{tabular}

At follow-up, employees were significantly more satisfied $(M=2.85, S D=0.11)$ with communication in the organization $(M=2.63, S D=0.0 .11, t(27)=-2.497, p=0.009, r=0.19)$. At baseline, they were significantly more satisfied $(M=3.29, S D=0.14)$ about their autonomy than at follow-up $(\mathrm{M}=3.13, \mathrm{SD}=0.15, t(26)=2.039, p=0.026, r=0.14)$. Also, employees were significantly more 
satisfied about participation at baseline $(M=4.05, S D=0.11)$ than at follow-up ( $M=3.77, S D=0.11$, $t(26)=2.945, p=0.007, r=0.22)$. All effects were small, however. Overall satisfaction scores were high at baseline $(M=7.89, S D=0.65)$ and follow-up $(M=7.90, S D=0.62)$ and did not change significantly over time $(p=0.44)$ (Table 6).

\section{Culture}

Willingness to change was more positively evaluated at follow-up than at baseline. Some respondents perceived this shift as a result of the project. As one explained,

"Last time you and I spoke to each other I had the feeling we [assistants and physicians] were working on different islands. And now the willingness to change the way we do things here has increased, just because of the project" (resident).

Not all respondents who noted more willingness to change linked this shift to the project, however.

Openness was also evaluated more positively at follow-up. When asked about the degree of openness, one respondent replied that

"it improved during the last year, based on the results [of the project]" (resident).

However, respondents distinguished between openness in different sections of the outpatient clinic (between the ORL section and the AC or between ORL and the VC, for instance). Some also perceived a hierarchical barrier to openness:

"In general, there is a good degree of openness [...] Yes, it differs [between sections], of course they [ORL, AC, VC] are both your colleagues, but these [VC employees] are your direct colleagues which makes it easier to discuss things with them." (VC assistant).

Summarized results are presented in Table 7. 


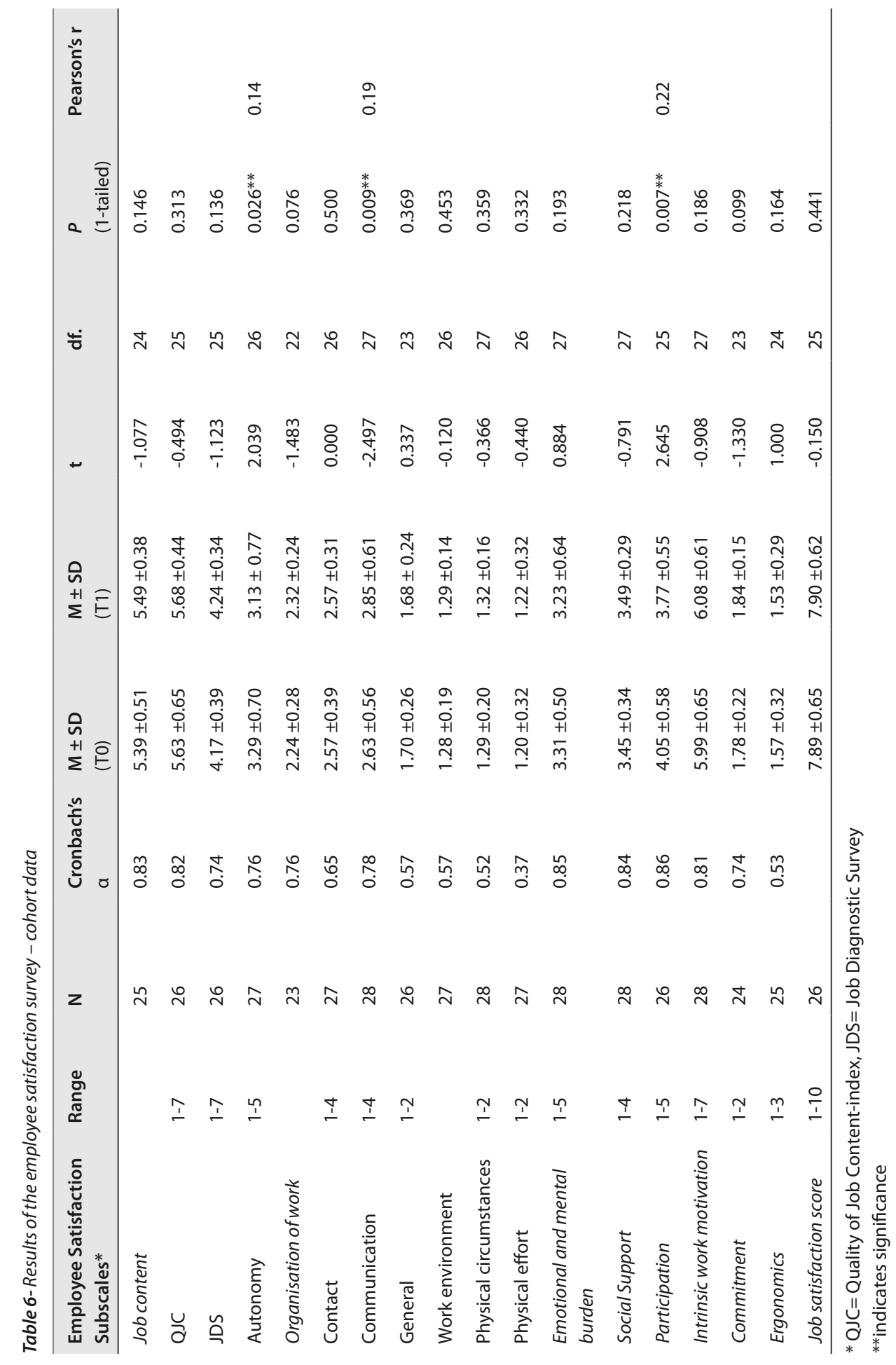




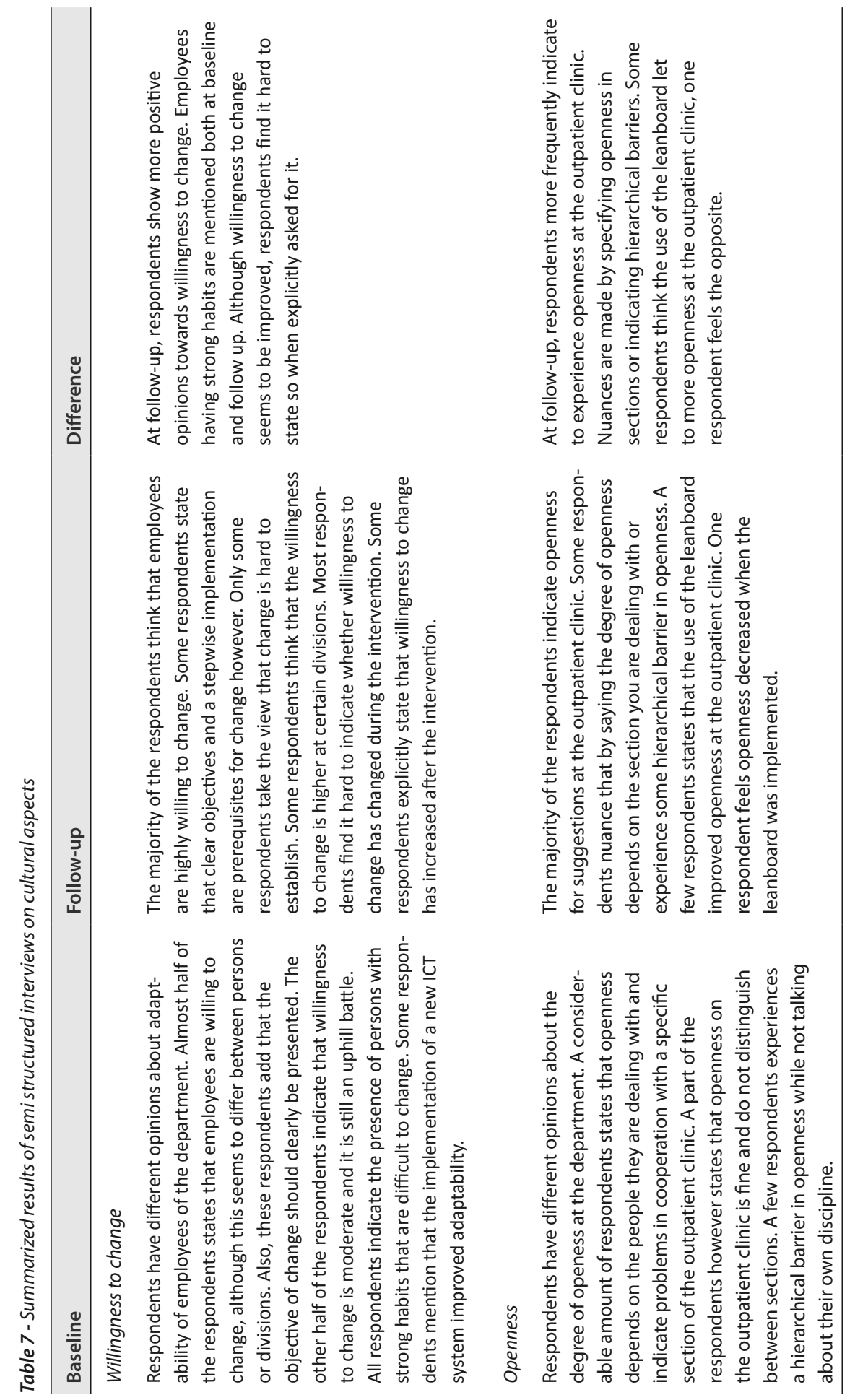




\section{DISCUSSION}

Although Lean is increasingly applied in healthcare settings, its implementation is not consistent ${ }^{7,11,12}$. Defining value for the customer in healthcare is subject to debate, and the effects of Lean on employees and the organizational culture are not well described ${ }^{7,16,32}$. To narrow that gap, this study evaluated Lean implementation with regard to the occurrence of waste and the effects on patient satisfaction, employee satisfaction and organizational culture.

During the project, improvements were made on 36 inefficiency issues. The kinds of waste that received the most remedial attention are waiting, defects and motion. After implementation, employees mentioned improvements in transportation, motion and waiting. Whereas defects were posted rather frequently on the Lean board, improvements in that regard were not mentioned. Since the points posted were not categorized as a specific type of waste, employees might not have perceived certain improvements as addressing defects, which possibly explains the difference. In addition, small improvements might not have been visible.

In general, patient satisfaction was high, both at baseline and follow-up, and the implementation of Lean did not lead to significant differences. Improvement efforts might not have been visible to the patient. Interviewing different groups of patients at baseline and follow-up might have influenced the results, although characteristics that are known to influence patient satisfaction did not differ significantly between the groups $s^{33,34}$. Furthermore, patient expectations $\mathrm{s}^{35}$ and a high score at baseline ${ }^{36}$ might also have influenced the results.

This study shows some small but significant effects of the implementation of Lean on employee satisfaction. Communication within the organization improved, whereas satisfaction with autonomy and participation decreased. Some employees believed that the Lean board promoted structured discussions of inefficiencies, possibly explaining their higher satisfaction with communication. Though not explicitly mentioned, autonomy might have decreased for some employees when standardized working procedures were introduced. Next, although Lean aims to involve frontline staff in the organization of processes, satisfaction with participation decreased after implementation. When presenting these findings in the project group, the members unanimously agreed that a learning effect among employees induced a more critical evaluation of their tasks - and participation opportunities - at follow-up. Furthermore, project group members believed that the shift in the problem-solving approach (employees now had to solve problems, not just report them to management) might have been negatively perceived. The results for employee satisfaction are similar to those found in another study evaluating the effect of Lean on employees ${ }^{6}$.

Improvements were noted regarding willingness to change and openness among employees. Some authors argue that focusing on socio-technical aspects of Lean - such as cultural change - will encourage superior performance in the organization ${ }^{16,37}$. And indeed, this study reveals some effect on cultural aspects. Yet it is too early to draw any firm conclusions, as the sociotechnical realm comprises more than willingness to change and openness. The results of this project do, however, confirm the importance of these aspects in Lean projects. 
In general, true adoption of Lean Thinking takes more than one year ${ }^{10}$. As seen in this project, employees need time to familiarize themselves with the principles. Their implementation implies a change in mind-set, both for employees and management. Notwithstanding the fact that Lean implementation was only in its beginning during this project, implementation showed to be successful. Despite the fact that success factors for implementation were not systematically asked for, some stimulating factors can be indicated. First, the involvement of all employees at the outpatient clinic most likely resulted in a higher willingness to change, a common sense of urgency and the use of tailored implementation strategies throughout improvement projects. Second, the involvement of the management level in the project group guaranteed the availability of manpower and financial resources for the project if necessary. Third, emphasis on creating an environment that stimulates creativity, vulnerability and openness during monthly lean sessions allowed for learning and unlearning existing routines ${ }^{38}$. Even though these factors are not exhaustive and many more barriers and facilitators for implementation are known in international literature ${ }^{39,40}$, they might be useful to consider in similar quality improvement initiatives.

\section{Limitations}

A possible influence of contingent factors cannot be excluded, as no control group was used in this study ${ }^{6,13}$. Such influence might show up in the results of the employee satisfaction survey, although those were in line with findings from other studies that included job satisfaction. Next, although the patient satisfaction survey is commonly used to measure quality of care, whether it is the right instrument for that purpose is intensely debated in the literature ${ }^{41-43}$. There is no consensus on which instrument is most valid, and patient satisfaction surveys are still frequently used. Even though other instruments are needed to evaluate the effects of Lean on patients, patient satisfaction surveys do allow for comparison between studies.

Using semi-structured interviews may have led to an underestimation of waste reduction, as some effects might not have been noticed. An in-depth evaluation using quantitative measures in addition to qualitative ones is necessary to determine the full effects of Lean. Semi-structured interviews were nonetheless useful in evaluating cultural changes. Therefore, it is worth considering their use to evaluate future Lean projects.

\section{CONCLUSION}

Implementation of Lean at an ORL outpatient clinic has led to numerous improvement efforts. Although employees experienced improvements at follow-up, waste was still present. Overall, both patient satisfaction and job satisfaction were high at baseline and had not changed significantly at follow-up. Employee satisfaction with communication in the organization rose significantly, whereas employee satisfaction with autonomy and participation was significantly lower. Willingness to change and openness among employees increased after the intervention, indicating effects beyond operational aspects like waste reduction.

The full effects of the intervention might be underestimated, as no quantitative measurement 
was performed and the time to follow-up was relatively short (one year). Longitudinal evaluation taking both operational and cultural aspects into account is recommended. Finally, as the first step of Lean is determining value to the customer, and the appropriateness of using patient satisfaction surveys to this end has been questioned, more insight is needed into patients' perspectives on quality of care.

\section{ACKNOWLEDGEMENT}

The authors would like to thank all employees working at the ORL outpatient clinic for their participation in both the project and its evaluation. In addition, the authors would like to thank all patients who participated in this study for their valuable contribution. 


\section{BIBLIOGRAPHY}

1. Berwick DM, Nolan TW, Whittington J. The triple aim: care, health, and cost. Health Aff 2008;27(3):759-769

2. Stabile M, Thomson S, Allin S, et al. Health Care Cost Containment Strategies Used In Four Other High-Income Countries Hold Lessons For The United States. Health Aff 2013;32(4):643-652

3. OECD. Health at a Glance: Europe 2012, 2012.

4. Institute of Medicine. Crossing the Quality Chasm: A New Health System for the 21st Century. Washington, DC: National Academies Press, 2001.

5. Institute of Medicine. To Err is Human: Building a Safer Health System. In: Linda T. Kohn, Janet M. Corrigan, Donaldson MS, eds. Washington D.C.: Institute of Medicine, 2000.

6. Holden RJ. Lean Thinking in emergency departments: a critical review. Ann Emerg Med 2011;57(3):265-278

7. Radnor ZJ, Holweg M, Waring J. Lean in healthcare: the unfilled promise? Soc Sci Med 2012;74(3):364-371

8. Young TP, McClean SI. A critical look at Lean Thinking in healthcare. Qual Saf Health Care 2008;17(5):382-386

9. Womack JP, Jones DT. Lean Thinking. Banish waste and create wealth in you corporation. New York: Free Press, 2003.

10. Tapping D, Luyster T, Shuker T. Value Stream Management. Eight steps to planning, mapping, and sustaining lean improvements. New York: Productivity Press, 2002.

11. Mazzocato $P$, Savage $C$, Brommels $M$, Aronsson $H$, Thor J. Lean thinking in healthcare: a realist review of the literature. Qual Saf Health Care 2010;19(5):376-382

12. Dellifraine JL, Langabeer JR, 2nd, Nembhard IM. Assessing the evidence of Six Sigma and Lean in the health care industry. Qual Manag Health Care 2010;19(3):211-225

13. Dickson EW, Anguelov Z, Vetterick D, Eller A, Singh S. Use of Lean in the Emergency Department: A Case Series of 4 Hospitals. Ann Emerg Med 2009;54(4):504-510

14. Helbig M, Helbig S, Kahla-Witzsch HA, May A. Quality management: reduction of waiting time and efficiency enhancement in an ENT-university outpatients' department. Bmc Health Serv Res 2009;9 doi: $2110.1186 / 1472-6963-9-21$

15. Lin SY, Gavney D, Ishman SL, Cady-Reh J. Use of lean sigma principles in a tertiary care otolaryngology clinic to improve efficiency. Laryngoscope 2013;123(11):2643-2648

16. Joosten T, Bongers I, Janssen R. Application of lean thinking to health care: issues and observations. Int J Qual Health Care 2009;21(5):341-347

17. KNO-heelkunde. Annual Report 2012. Department of Otorhinolaryngology [in Dutch]. Maastricht: Maastricht Universitair Medisch Centrum, 2012.

18. Lean Sigma Yellow Belt Certificate. Maastricht: Medtronic inc., 2011. 
19. Patton M. Qualitative evaluation and research methods. Beverly Hills, CA: Sage, 1990.

20. Kleefstra SM, Kool RB, Veldkamp CM, et al. A core questionnaire for the assessment of patient satisfaction in academic hospitals in The Netherlands: development and first results in a nationwide study. Qual Saf Health Care 2010;19(5):e24

21. Houkes I, Janssen PP, de Jonge J, Bakker AB. Personality, work characteristics, and employee well-being: a longitudinal analysis of additive and moderating effects. J Occup Health Psychol $2003 ; 8(1): 20-38$

22. Houkes I, Janssen PPM, de Jonge J, Nijhuis FJN. Work and individual determinants of intrinsic work motivation, emotional exhaustion, and turnover intention: A multi-sample analysis. Int J Stress Manage 2001;8(4):257-283

23. Berndsen MB. Checklist voor de beeldschermwerkplek. Alphen aan den Rijn: Samsom, 1993.

24. Gründemann RWM, Smulders PGW, Winter CRd. VAG. Vragenlijst Arbeid en Gezondheid. Handleiding. Lisse: Swets \& Zeitlinger, 1993.

25. Fereday J, Muir-Cochrane E. Demonstrating Rigor Using Thematic Analysis: A Hybrid Approach of Inductive and Deductive Coding and Theme Development. International Journal of Qualitative Methods 2006;5(1):80-92

26. NVivo qualitative data analysis software, version 10 [program]. QSR International, 2012.

27. Miles M, Huberman M. Qualitative data analysis: An expanded sourcebook (2nd ed.). Thousand Oaks, CA: Sage, 1994.

28. Denzin NK. Interpretive Interactionism. Newbury Park, CA: Sage Publications, 1989.

29. Declaration of Helsinki, Ethical Principals for Medical Research Involving Human Subjects. Secondary Declaration of Helsinki, Ethical Principals for Medical Research Involving Human Subjects 2008. World Medical Association Website. http://www.wma.net/en/30publications/10policies/b3/index. html. Published June, 1964. Updated October, 2013. Accessed March 16, 2015.

30. Ministerie van Volksgezondheid, Welzijn en Sport. The Dutch Medical Research (human subjects) Act [In Dutch]. Den Haag, 2014.

31. Ogrinc G, Mooney SE, Estrada C, et al. The SQUIRE (Standards for QUality Improvement Reporting Excellence) guidelines for quality improvement reporting: explanation and elaboration. Qual Saf Health Care 2008;17 Suppl 1:i13-i32

32. DelliFraine JL, Wang Z, McCaughey D, Langabeer JR, 2nd, Erwin CO. The use of six sigma in health care management: are we using it to its full potential? Qual Manag Health Care 2013;22(3):210-223

33. Hall JA, Dornan MC. Patient sociodemographic characteristics as predictors of satisfaction with medical care: a meta-analysis. Soc Sci Med 1990;30(7):811-818

34. Hekkert KD, Cihangir S, Kleefstra SM, van den Berg B, Kool RB. Patient satisfaction revisited: a multilevel approach. Soc Sci Med 2009;69(1):68-75 
35. Crow R, Gage H, Hampson S, et al. The measurement of satisfaction with healthcare: implications for practice from a systematic review of the literature. Health Technol Assess 2002;6(32):1-244

36. Moret L, Nguyen JM, Pillet N, Falissard B, Lombrail P, Gasquet I. Improvement of psychometric properties of a scale measuring inpatient satisfaction with care: a better response rate and a reduction of the ceiling effect. BMC Health Serv Res 2007;7:197

37. Collar RM, Shuman AG, Feiner S, et al. Lean management in academic surgery. J Am Coll Surg 2012;214(6):928-936

38. Rushmer R, Davies HT. Unlearning in health care. Qual Saf Health Care. 2004;13 Suppl 2:ii10-15

39. Grol R, Grimshaw J. From best evidence to best practice: effective implementation of change in patients' care. Lancet. 2003;362(9391):1225-1230.

40. Chaudoir SR, Dugan AG, Barr CHI. Measuring factors affecting implementation of health innovations: a systematic review of structural, organizationals, provider, patient and innovation level measures. Implementation Science. 2013;8(22).

41. Coyle J, Williams B. Valuing people as individuals: development of an instrument through a survey of person-centredness in secondary care. J Adv Nurs 2001;36(3):450-459

42. de Silva D. Helping measure person-centred care. A review of evidence aboutcommonly used approaches and tools used to help measure person-centred care. London: the Health Foundation, 2014.

43. Tsianakas V, Maben J, Wiseman T, et al. Using patients' experiences to identify priorities for quality improvement in breast cancer care: patient narratives, surveys or both? BMC Health Serv Res 2012;12:271 


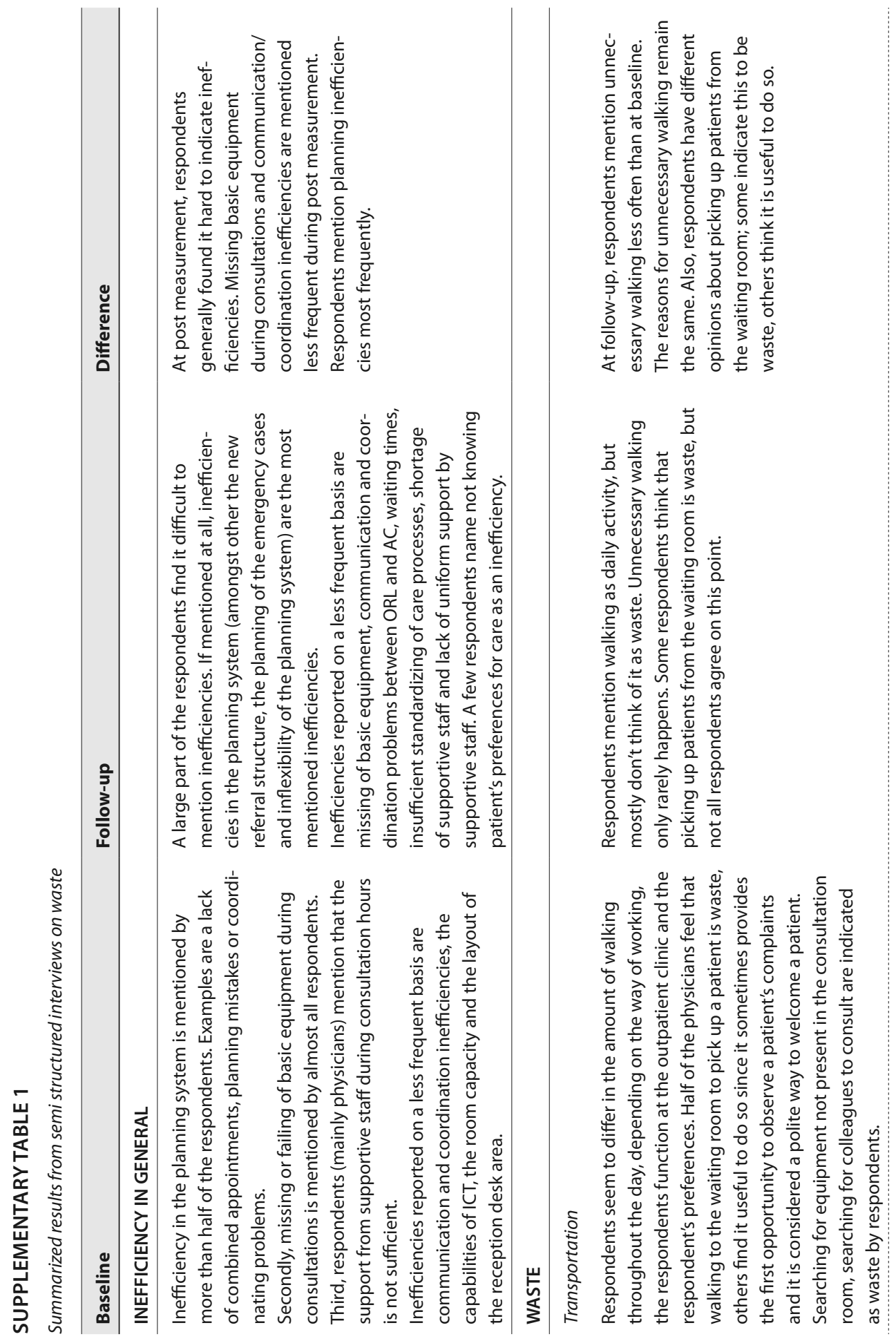




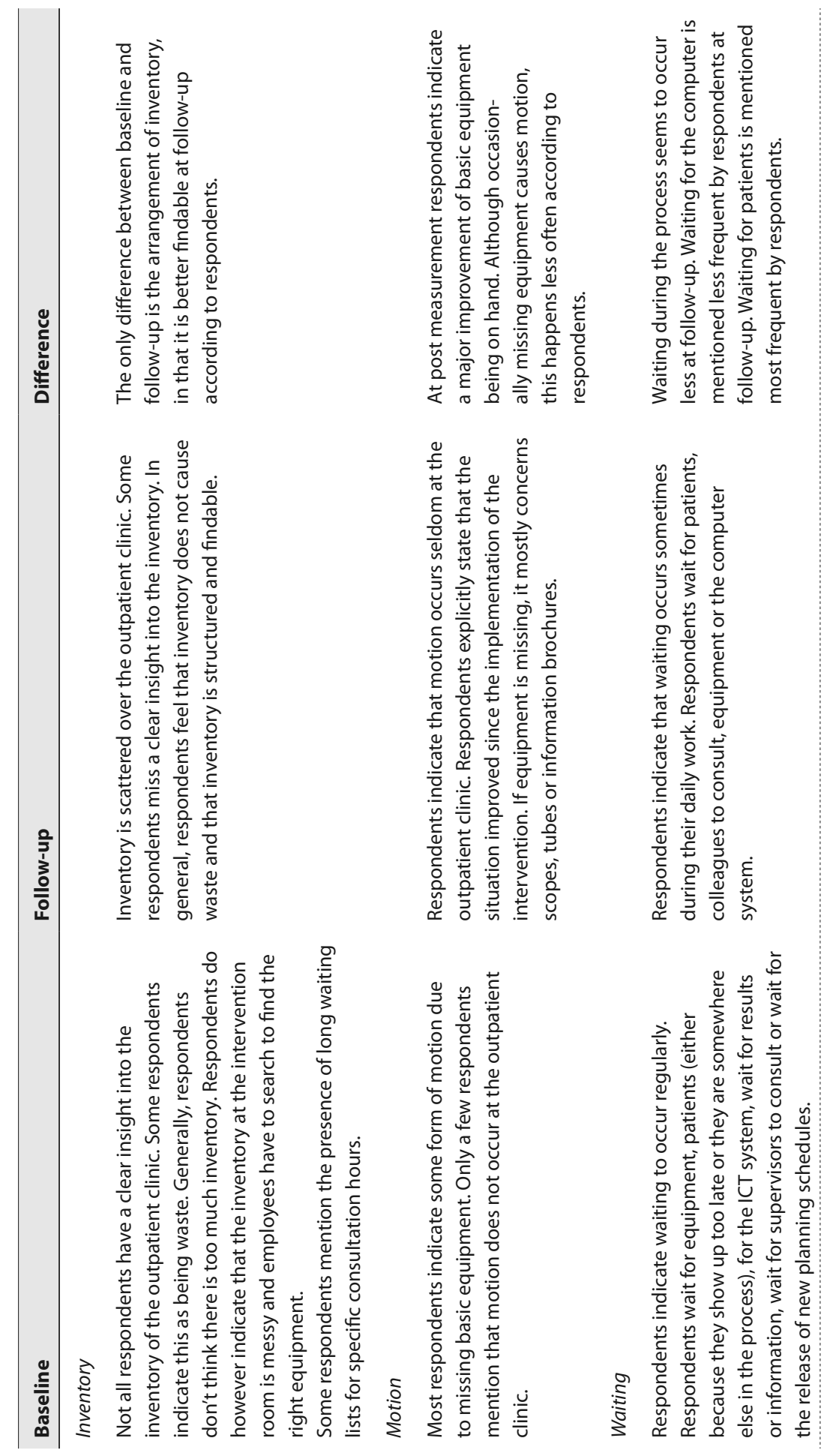




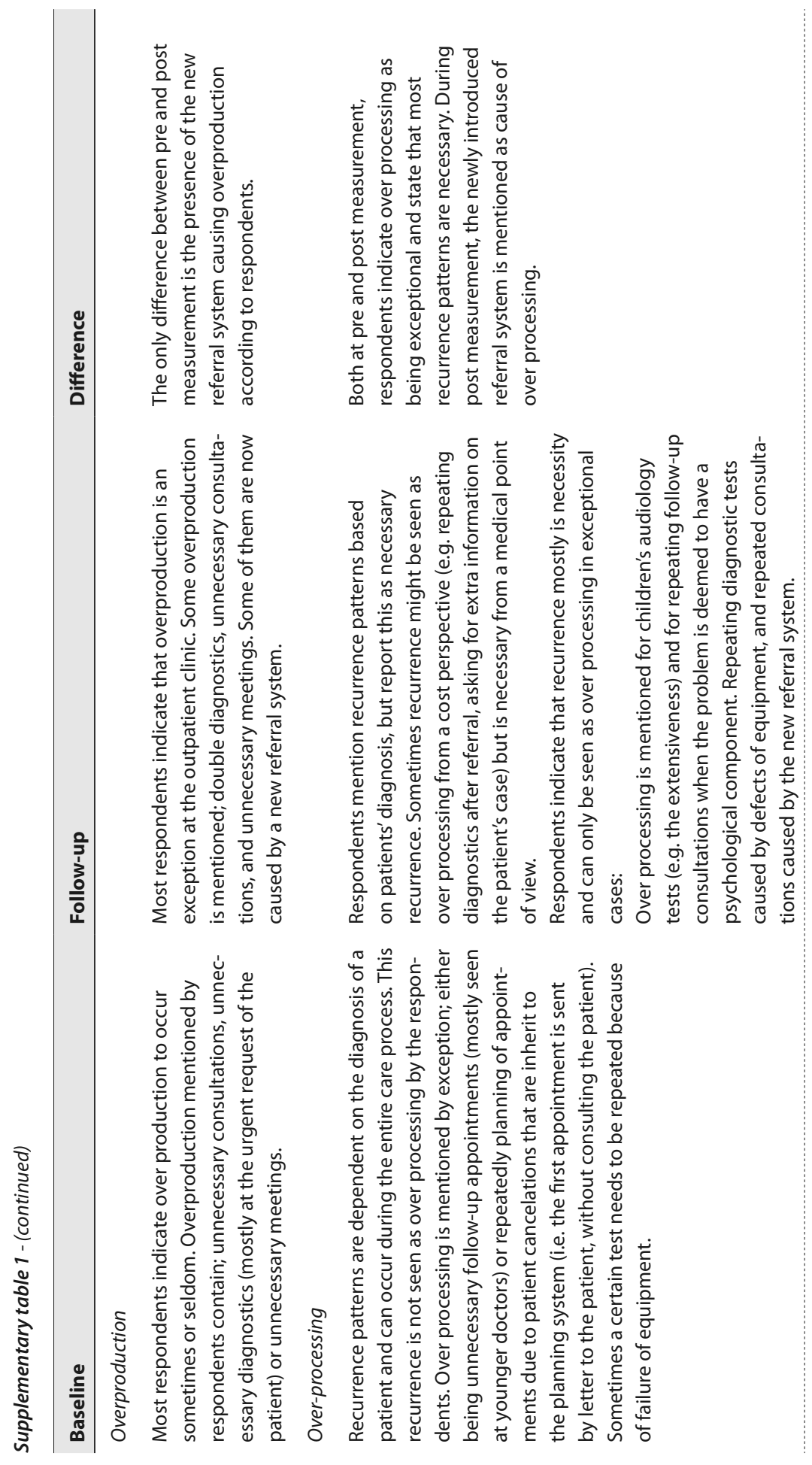










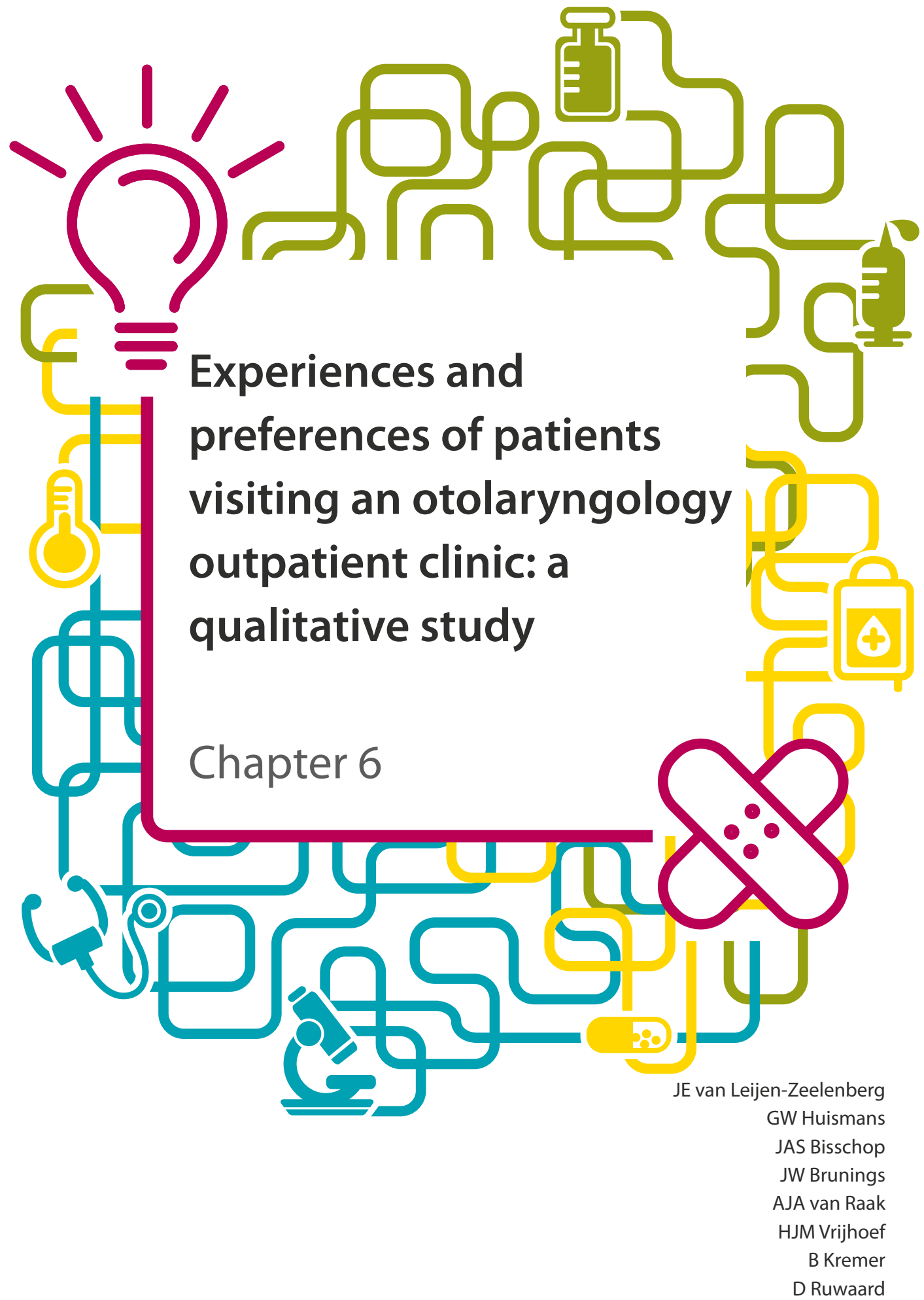

Health Expectations, 2015 (epub ehead of print) 


\section{ABSTRACT}

\section{Background}

Patient-centred care has received considerable attention in the last few decades, but the patients' perspective remains underexposed. This study reports on an in-depth evaluation of patients' experiences and preferences at an otorhinolaryngology outpatient department.

\section{Method}

Qualitative research was conducted on patients' experiences and preferences at an otorhinolaryngology outpatient department in an academic hospital. The study comprised two phases. First, semi-structured interviews were held with 22 patients. Second, results from the interviews were verified and deepened in a focus group $(\mathrm{N}=7)$.

\section{Results}

Overall, experience with patient-centred care was positive at the outpatient department. Three of the six dimensions of patient-centred care predominated in the interviews and the focus group: information, communication and education; coordination and integration of care; respect for patients' values, preferences and expressed needs. The negative experiences were mostly in these dimensions. The dimensions physical comfort and involvement of family and friends were of lesser significance. Opinion on emotional support - relieving fear and anxiety differed as to whether this was the responsibility of the doctor or the patient.

\section{Conclusion}

Qualitative research provided a deeper understanding of patients' experiences and preferences at an otorhinolaryngology outpatient department. Such an in-depth evaluation can be useful in the transition towards patient-centred care. 


\section{INTRODUCTION}

Patient-centred care (PCC) has received considerable attention in the last few decades and is seen as a key element of care delivery'. The Institute of Medicine (IoM) defined PCC as an important area for improvement in healthcare ${ }^{2}$. Apart from being a goal in itself, PCC also leads to improved health outcomes ${ }^{3}$. Besides lowering the costs per patient by reducing diagnostic testing, referrals and other health resource utilization, it improves patient well-being. PCC also increases adherence to treatment and is associated with improved quality of life $e^{2-9}$.

Despite the interest in PCC, this concept has not been clearly defined in the literature ${ }^{3,8,10-12}$. The IoM ascribes six dimensions to PCC: (1) respect for patients' values, preferences and expressed needs; (2) information, communication and education; (3) coordination and integration of care; (4) emotional support - relieving fear and anxiety; (5) physical comfort; and (6) involvement of family and friends ${ }^{2}$. (See Table 1.)

Table 1 - Six dimensions of Patient-Centred Care (PCC) ${ }^{2}$

\begin{tabular}{|c|c|}
\hline Dimension & Definition \\
\hline $\begin{array}{l}\text { Respect for patients' values, } \\
\text { preferences and expressed needs }\end{array}$ & $\begin{array}{l}\text { Health care is patient centred when precisely responding to a } \\
\text { patient's preferences, needs and wishes. }\end{array}$ \\
\hline $\begin{array}{l}\text { Information, communication and } \\
\text { education }\end{array}$ & $\begin{array}{l}\text { Health care is patient centred if information on diagnosis, } \\
\text { prognosis and treatment is responsive, attentive, trustworthy } \\
\text { and tailored to the individual's needs. }\end{array}$ \\
\hline Coordination and integration of care & $\begin{array}{l}\text { PCC ensures smooth care transitions to other settings, accurate } \\
\text { and timely information transfer to the right persons. }\end{array}$ \\
\hline $\begin{array}{l}\text { Emotional support - relieving fear } \\
\text { and anxiety }\end{array}$ & $\begin{array}{l}\text { PCC reacts to any fear, anxiety or other negative feelings caused } \\
\text { by the illness or injury to both the patient and one's family. }\end{array}$ \\
\hline Physical comfort & $\begin{array}{l}\text { PCC provides timely and tailored support to experienced } \\
\text { shortness of breath, pain or other discomfort of a patient. }\end{array}$ \\
\hline Involvement of family and friends & $\begin{array}{l}\text { In PCC, family and friends of patients are - if appropriate - to be } \\
\text { involved in decision making, are supported as caregivers and } \\
\text { respected and welcomed in the care delivery setting. }\end{array}$ \\
\hline
\end{tabular}

As shown in a recent study, these dimensions adequately reflect patients' perceptions of PCC and their overall rating of care ${ }^{12}$. The loM describes PCC as "delivering care respectful of and responsive to individual patient preferences, needs, values, and ensuring that patient values guide all clinical decisions" (p.48)2. From that perspective, efforts to deliver PCC would have to be based on knowledge about patients' expectations ${ }^{13}$. But insight into their preferences and experiences is not obtained through straightforward questioning ${ }^{14}$. Recently, an extensive review was published on the wide range of tools to measure PCC, some from a holistic perspective, others focused on its subcomponents ${ }^{1}$. While structured surveys are the most commonly used tool, questions have been raised about the usefulness of their results ${ }^{1,15,16}$. Instead, in-depth qualitative analysis has proved to be more helpful for making improvements than survey data alone $8,14,15,17,18$. 
Although PCC is important in the field of otorhinolaryngology (ORL), to our knowledge no in-depth qualitative information has been gathered about patients' perspectives on PCC. Recent studies based on surveys have revealed differences in patient experience between subgroups of ORL patients. One implication is that their particular needs should be taken into account ${ }^{13,19-21}$. In a more general sense, PCC means understanding a patient's unique needs and preferences in order to deliver care that is respectful to those preferences. That would entail obtaining in-depth knowledge for each patient. Some dimensions of PCC are more context-specific, however, namely physical comfort and coordination and integration of care. Accordingly, PCC might also be measured for specific groups, such as ORL patients. Understanding their preferences and needs could facilitate efforts to improve the quality of care by explicitly defining PCC for this patient group. Subsequently, adequate outcome measures could be defined to evaluate the shift towards PCC 22,23 . The aim of the present study was to explore the patients' experiences and preferences at ORL outpatient clinics in terms of the six domains of PCC.

\section{METHOD}

\section{Study design}

To ensure quality when reporting the study, the COREQ checklist was used ${ }^{24}$. It concerns the use of interviews and focus groups for collecting data in qualitative research. The items on the checklist are grouped into three domains: (1) research team and reflexivity; (2) study design; and (3) analysis and findings.

This study used a qualitative research design to determine the experiences and preferences of patients visiting the ORL outpatient clinic of the Maastricht University Medical Centre+ (MUMC+) in the Netherlands. At the clinic, over 20,000 patients are seen each year. The study was performed in two phases.

First, 22 semi-structured interviews were conducted with patients visiting the ORL outpatient clinic. The questions were based on the six dimensions of PCC as defined by the loM ${ }^{2}$. On each dimension, patients were asked to share their experience at the ORL outpatient clinic and state their preferences for the future. The questions (listed in appendix 1 ) were tested in a pilot before the start of the project. The interviews were conducted by a male researcher (GWH) who had a Master's degree and was a senior medical intern. The researcher, who was trained by an experienced interviewer, let the participants elaborate freely on the topics. After each interview, the researcher wrote down his perceptions, thereby creating field notes.

Second, a focus group (FG) comprising seven of the interview respondents was held in order to verify and deepen the findings. Three out of the six dimensions of PCC were discussed in the FG: (1) coordination and integration of care; (2) communication, information and education; and (3) emotional support. These were either the dimensions most extensively discussed during the semi-structured interviews or the ones that evoked opposite opinions. For each dimension, at least one statement was formulated on which the respondents could elaborate. In total, six statements were discussed during the FG (appendix 2). Three concerned coordination and 
integration of care; two concerned communication, information and education; and one statement referred to emotional support. At the end of the session, respondents were asked where they would give priority for improvement. The FG was convened by two researchers (GWH and JVLZ), who let the participants freely elaborate on each statement and then summarized the outcomes. One of the researchers was male, the other female. Both hold a Master's degree; one researcher was a senior medical intern at the time of the study $(\mathrm{GWH})$, the other a PhD student (JVLZ). Both were well trained in interviewing.

\section{Sampling strategy}

Maximum variation sampling was used to select respondents $\mathrm{s}^{25}$. Considering the importance of individual preferences and needs in PCC, it is crucial to take the heterogeneity among patients into account. Maximum variation sampling is conducive to searching for patterns shared among a heterogeneous group ${ }^{25}$. As the ORL outpatient clinic considers its population to be heterogeneous, maximum variation sampling was very suitable.

To enhance the probability of a representative selection of respondents, three criteria on which they should vary were formulated. Respondents had to vary in terms of: (1) sub-specialism (ear, nose, throat, balance); (2) degree of case complexity (simple vs. complex); and (3) age category (either below or above 50 years old). Persons with head and neck cancer were excluded from the study, as they are diagnosed and treated at the hospital's oncology centre. There, the organization of care, the physical environment and personnel differ from the outpatient clinic. These characteristics are likely to influence patients' answers to some of the questions of the interviews - questions directly related to organization of care and physical comfort, for instance. Therefore, these oncology patients' opinions would not reflect the experiences of the ORL outpatient clinic. As no standards exist for simple or complex cases within the ORL outpatient clinic, the degree of complexity was determined by the medical head of the department (BK). In addition, since the respondents had to be able to share experiences, patients were only included if they had visited the ORL outpatient clinic more than four times in the last two years.

Patients were deemed eligible for inclusion if they were scheduled for a visit to the ORL outpatient clinic. Two researchers (GWH, JWB) scanned the consultation calendar and selected eligible respondents, who were then approached by telephone. After consenting verbally to participate, respondents were sent information about the study by regular mail or e-mail. Interviews were scheduled either right before or after their consultation at the ORL outpatient clinic. Patients were asked not to mention their participation in order to keep the doctor blind to the study. The interviewer introduced himself as the researcher (not the care provider) to the patients once they reported to the reception desk. At that point, the researcher asked for written consent.

After the interview, patients were asked to participate in the FG. If willing, they were sent information by regular mail or e-mail about the date and content of the FG. At the start of the session, all participants were asked to give their written consent. Both researchers introduced themselves 
at the beginning of the $\mathrm{FG}$, providing the participants with information on their professional background, the aims of the study and the procedure. The semi-structured interviews and the FG were held in the MUMC+. The study protocol was assessed by the Medical Ethical Commission of the MUMC+, but full consideration was deemed unnecessary under Dutch Law.

\section{Analysis}

A template approach ${ }^{26}$ was chosen for the coding process. The codebook was based on the key elements of PCC as defined by the loM. The interviews and the FG sessions were recorded and transcribed verbatim to increase validity ${ }^{27}$. The respondents did not give feedback on the transcripts. Two researchers (GWH, JVLZ) independently coded one of the transcripts and discussed the codebook until consensus was reached. The remaining 20 interviews ${ }^{1}$ were then coded by one reviewer $(\mathrm{GWH})$ using NVivo ${ }^{28}$. The answers were inserted in a data matrix in which the rows give the respondents and the columns contain the concepts from the codebook. Then two researchers $(\mathrm{GWH}, \mathrm{JB})$ independently transformed the 'thick descriptions' into 'thin descriptions' - i.e., summarizing respondents' answers ${ }^{27}$. Next, the thin descriptions were converted into summaries per concept by two researchers independently $(\mathrm{GWH}, \mathrm{JB})$. These summaries were discussed until consensus was reached. If necessary, a third researcher (JVLZ) joined the discussion and made a decision. The answers given by respondents in the FG were entered into a data matrix, with the rows showing the topics and the columns containing the answers. Two researchers (GWH, JVLZ) independently transformed the 'thick descriptions' into 'thin descriptions' which were then transformed into summaries. The summaries were discussed until consensus was reached. Finally, conclusions from the semi-structured interviews and the FG were compared by two researchers (GWH, JVLZ) to identify possible discrepancies, similarities and deeper insights.

\section{RESULTS}

In total, 52 patients were approached and 25 agreed to participate in the study (response rate $48 \%)$. As those refusing were not obliged to say why, the reasons for not participating in the study are unknown. Three respondents dropped out after being enrolled: one felt ill on the day of the interview; one did not show up; and one experienced scheduling problems that prevented him from joining in. No repeat interviews were carried out. Out of the 22 respondents that did take part in the study, six attended the FG. One was accompanied by his wife, which brought the total up to seven FG members. Respondents' characteristics are summarized in Table 2 . The duration of the interviews varied between 21 and 69 minutes (median 52 minutes), mostly depending on the complexity of the respondent's medical case (the more complex the case, the longer the interview). An overview of the main results from both the semi-structured interviews and the FG is presented in Table 3.

1. During the analysis, the recording of respondent no. 6 proved to be unusable because of noise, so this respondent was not included in the final analysis. 
Table 2 - Basic characteristics of respondents $(n=22)$

\begin{tabular}{|c|c|c|c|c|c|}
\hline Nr. & Specialism & Degree of complexity & Sex & Age & Focus Group participation \\
\hline 1 & Vestibulogy & high & $\mathrm{F}$ & 53 & No \\
\hline 2 & Laryngology & high & $\mathrm{F}$ & 57 & No \\
\hline 3 & Otology & low & M & 57 & No \\
\hline 4 & Laryngology & low & $\mathrm{F}$ & 59 & Yes \\
\hline 5 & Otology & high & $\mathrm{F}$ & 24 & Yes \\
\hline 6 & Rhinology & low & M & 10 +father & No \\
\hline 7 & Benign tumours & high & $\mathrm{F}$ & 37 & No \\
\hline 8 & Otology & low & M & $8+$ mother & No \\
\hline 9 & Otology & high & M & 57 & Yes \\
\hline 10 & Laryngology & high & $\mathrm{F}$ & 46 & No \\
\hline 11 & Otology & high & M & 54 & Yes \\
\hline 12 & Otology & high & $\mathrm{F}$ & 63 & No \\
\hline 13 & Vestibulogy & low & $\mathrm{F}$ & 68 & Yes \\
\hline 14 & Vestibulogy & low & M & 65 & Yes \\
\hline 15 & Benign tumours & high & M & 48 & No \\
\hline 16 & Laryngology & high & M & 64 & No \\
\hline 17 & Rhinology & low & $\mathrm{F}$ & 76 & No \\
\hline 18 & Rhinology & low & $\mathrm{F}$ & 18 & No \\
\hline 19 & Vestibulogy & low & $\mathrm{F}$ & 60 & No \\
\hline 20 & Vestibulogy & high & $\mathrm{F}$ & 29 & No \\
\hline 21 & Otology & high & $\mathrm{F}$ & 68 & No \\
\hline 22 & Rhinology & low & M & 52 & No \\
\hline
\end{tabular}

\section{Respect for patients' values, preferences and expressed needs}

On the dimension respect for patients' values, preferences and expressed needs, two main topics emerged from the analysis. First, the majority of the respondents were satisfied with how their preferences were being dealt with. One respondent highlighted the willingness of the doctors to cooperate:

"The doctor even reserved a different treatment room for me, only because I wanted to see the lesion myself. ... That was pleasant. Now I have evidence it is not something my mind just made up." (respondent no. 9) 
Three respondents reported a negative experience in which their preference could not be complied with; the preference concerned refusing appointments with specific physicians. Five respondents felt they had to be assertive at the ORL outpatient clinic in order to get respect for their preferences and needs. Second, with the exception of two respondents, all felt free to ask questions during consultations. Two respondents were less satisfied and felt that there was no opportunity to ask questions or that the answers to their questions were not targeted to the patient's level of knowledge.

Respondents in the FG stated that it is important to get an explanation when an expressed preference is not being complied with. They also found that being able to ask questions is a very important issue. Raising questions could be stimulated by encouraging the clinician to show a calm attitude and ask the patient to summarize the information.

\section{Information, communication and education}

Within the dimension information, communication and education, five main topics emerged from the analysis. First, with exception of one person, all respondents were satisfied with their doctorpatient communication and described it as good and clear. This was verified by the respondents in the FG.

Second, respondents described communication with the administrative staff of the outpatient clinic as friendly, helpful and respectful. Some reported communication problems caused by planning mistakes. Nonetheless, those respondents noted a willingness to solve the problems once identified. The respondents in the FG considered a problem-solving approach to be of great importance in communication with the administrative staff.

Third, respondents reported some negative experiences with external communication, for instance with their GP. Four respondents mentioned external communication to be unclear, untimely or non-existent.

Fourth, all respondents thought their doctor was well prepared at the consultation and they experienced good internal communication. This assessment was verified by the respondents in the FG. During the semi-structured interviews as well as during the FG, respondents mentioned that adding multimedia to the patient file - like pictures or drawings - would assist doctors in being prepared and transferring information among each other. As one respondent noted,

"I feel that a doctor who doesn't know me really faces difficulties in getting an image of what he actually sees in my ear .... I think the patient file should be expanded. It shouldn't only contain written text but could be completed with pictures or movies." (respondent no. 5)

Finally, respondents reported on the information they received at the outpatient clinic, some saying they would have appreciated more guidance on, for instance, procedures or tests. In the FG, the information on logistical procedures in the hospital was considered extensive. Respondents mentioned that a brief explanation of logistical procedures would be helpful. As one FG member stated, 
"It is quite a lot that you need to do when they schedule you for surgery. I'm experienced by now, but if I weren't I'm sure I would have felt lost. What to do with all this information? If they would give a quick step-by-step explanation of what to expect, you'd feel better prepared." (respondent no. 5, FG)

\section{Coordination and integration of care}

Four topics emerged within the dimension coordination and integration of care, namely planning, alternating doctors, access times and waiting in the waiting room.

With regard to planning, respondents experienced inflexibility in the planning system. Some were confronted with the impossibility of planning more than three months in advance, others with the impossibility of making combined appointments (at both the ORL outpatient clinic and the Audiology Centre, for instance) on short notice. As one respondent recounted,

"Indeed, when did I call for the last time? I don't remember. Anyway, after the first phone call I tried two more times. But the schedule still wasn't there. And then, finally in November, the schedule was ready. That is quite short notice since it is now the beginning of December." (respondent no. 20)

The impression of inflexibility was confirmed in the FG. Apart from the problems in the planning system, respondents experienced a efficient care process at the outpatient clinic. This too was confirmed by those in the FG.

Seeing different doctors at subsequent appointments was an important topic in most of the semi-structured interviews as well as in the FG. Negative experiences due to alternating doctors were reported by some patients, especially those with complex cases. As one respondent put it,

"At almost every appointment I saw a different resident. (...) I had the feeling they didn't really know what to do with my case, I think that was the worst part. I needed to tell the same story over and over again. And every time that day's supervising doctor was called for a second opinion ...." (respondent no. 7)

The topic of alternating doctors was discussed in more depth at the FG session. In the end, respondents thought that the reason why alternating doctors was a problem is that patients need some kind of trusted representative to build a relationship with. One respondent in the FG explained it thus:

"I don't really mind the principle of seeing alternating doctors. I can see they all got the information from the last one. So essentially I am not against seeing alternating doctors. But not if each time you see a different doctor. You bond with your doctor. I like that." (respondent no 11)

Members of the FG believed that being screened by a staff member for the first time - for complex cases - and knowing that this person is available as a supervisor for upcoming appointments would already improve the procedure. 


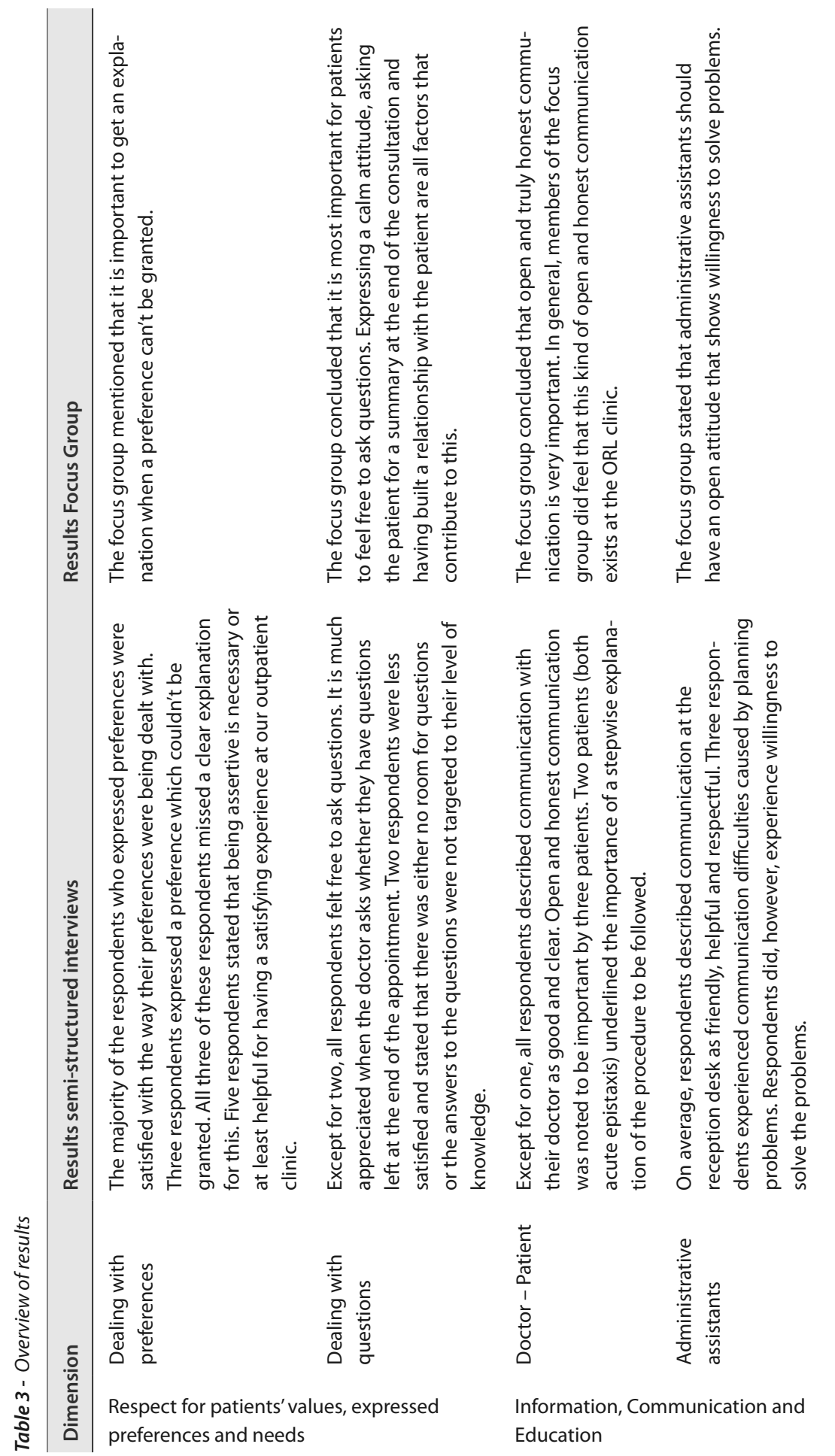




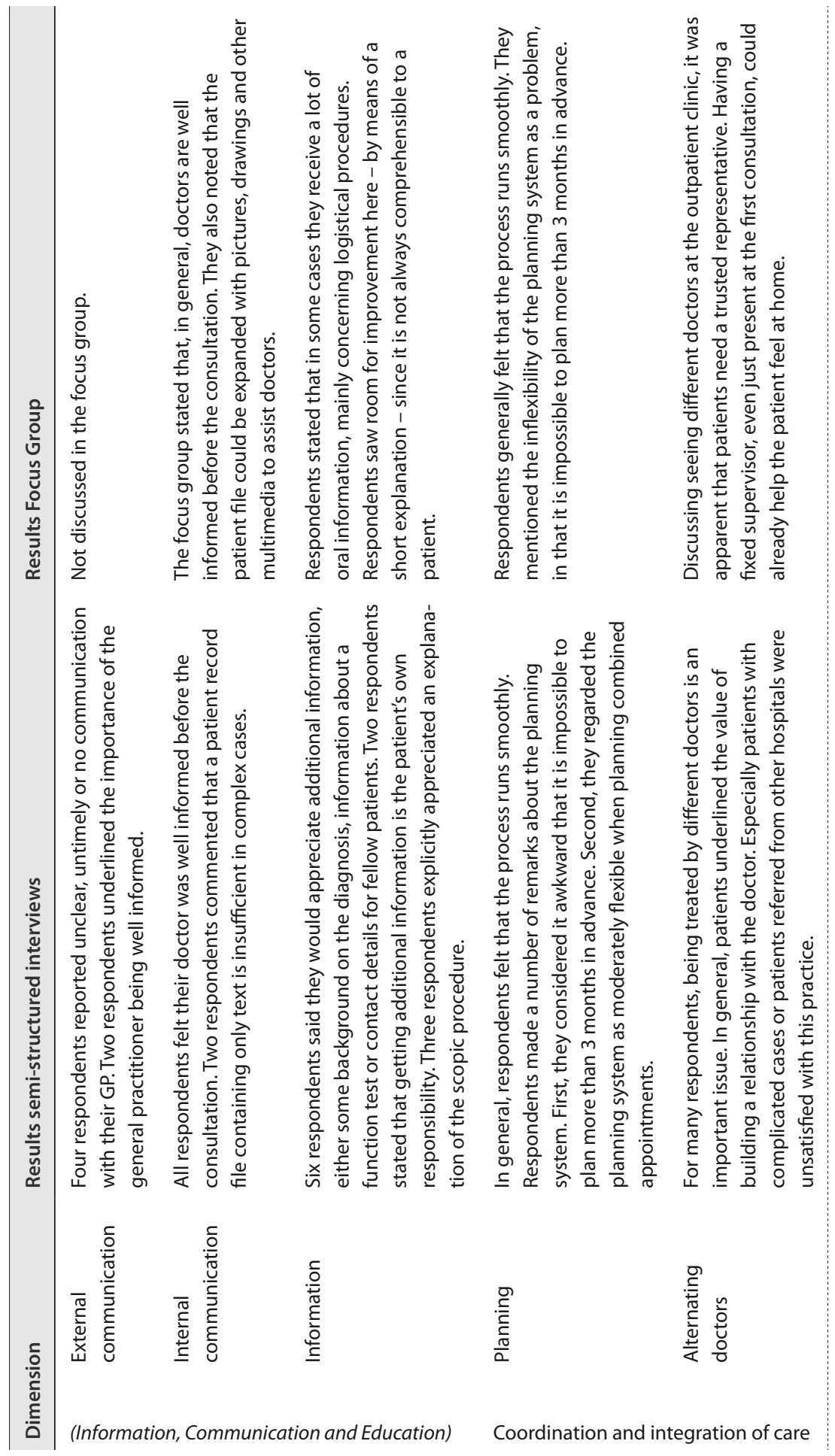









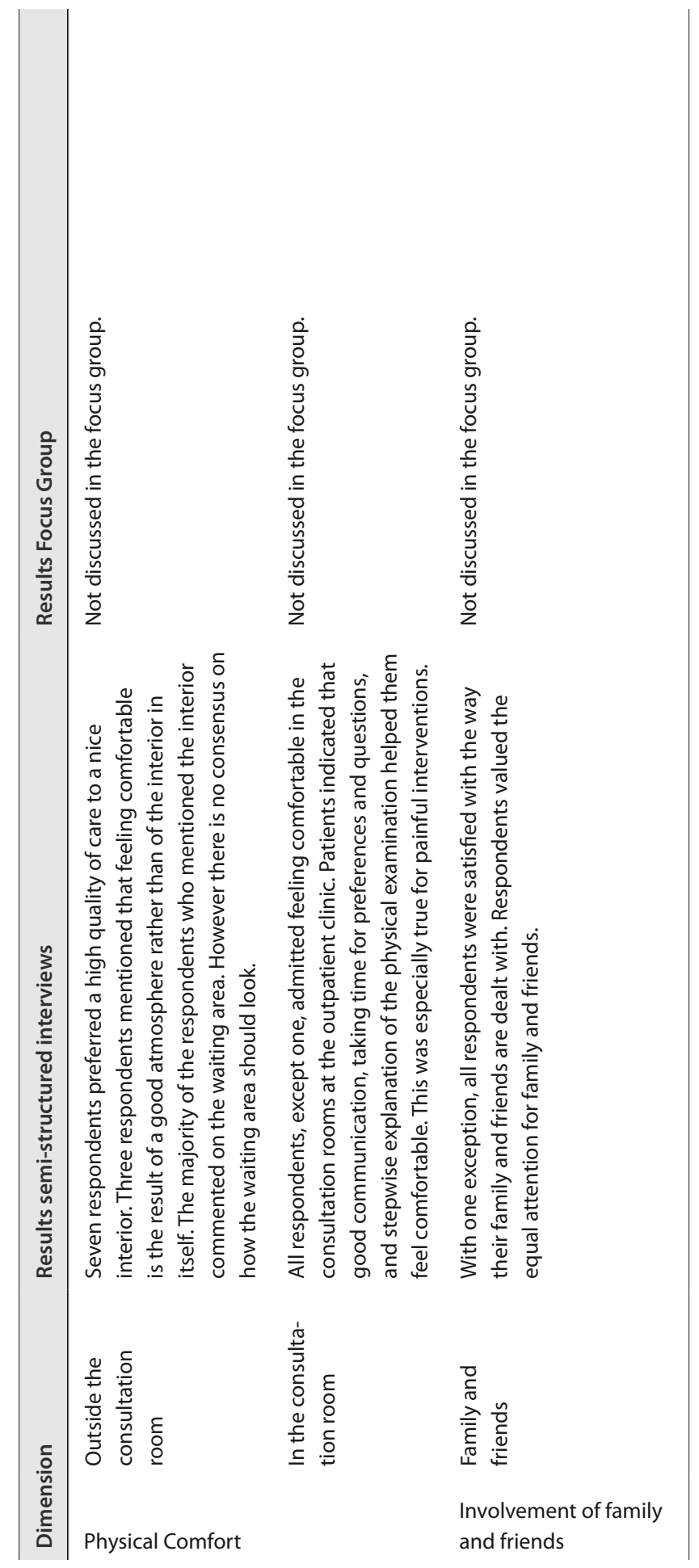


Only two respondents mentioned negative experiences with access times; both concerned vestibular cases. When access was discussed in the FG, respondents mentioned that waiting times can be problematic throughout the entire process. Two nuances were made on this point, however. First, respondents thought that waiting times are more problematic for patients with high ADL restrictive and complex symptoms than they are for patients with low ADL restrictive and relatively simple symptoms. Second, respondents thought that waiting times would be less problematic if they were to be explained.

In general, respondents felt that waiting times on the day of the appointment were short.

\section{Emotional support -- relieving fear and anxiety}

A distinction was made between emotional and psychological support, on the one hand, and social support on the other. Half of the respondents stated that little or no attention was given to emotional and psychological support. For the majority of these respondents, its neglect was in line with their expectations. Three respondents (all vestibular patients) reported incongruence between the expected support and the support received. One respondent said that

\section{"Until now I haven't experienced any emotional support. I do see a psychiatrist and psychol- ogist. It was the GP, however, who referred me when I developed other problems as well." (respondent no. 20)}

During the FG, respondents highlighted the importance of emotional and psychological support. Furthermore, opinion in the FG differed as to whether enough emotional support was provided and if emotional support should be initiated by the doctor.

With respect to social support, the majority of the respondents felt that the doctor gave sufficient attention to the social impact of their disease. Five reported a negative experience, three of whom were vestibular patients and two laryngology patients. This topic was not addressed in the FG.

\section{Physical comfort}

For the dimension physical comfort, a distinction was made between time spent in the consultation room and outside of it. In general, comfort outside the consultation room was considered less important. Some even felt that physical comfort outside the waiting room is influenced by the atmosphere at the outpatient clinic rather than by the features of the interior itself. As one patient put it,

"It is more the prospect of meeting nice and friendly people. You know a hospital is for medical reasons as sober as it is. We are not here to be cosy." (respondent no. 9)

With respect to physical comfort within the consultation room, almost all patients reported positive experiences. Good communication, taking time for patients' physical preferences and giving a stepwise explanation of the physical examination were said to contribute to the patient's physical comfort. 


\section{Involvement of family and friends}

Almost all respondents were positive about the involvement of family and friends. In general, they felt that these parties are given equal attention during the appointment. Only one respondent reported a negative experience, stating that her mother was completely ignored during the consultation. This dimension was not discussed in the FG.

\section{DISCUSSION}

Relatively little is known about experiences and preferences for PCC at ORL outpatient departments. Some recent studies in that setting emphasize the unique needs of certain groups within the ORL population and the importance of further research into patient experiences in ORL ${ }^{19-21}$. The present report provides deeper insight into patients' experiences with and preferences for PCC in ORL. Specifically, it offers three important insights.

First, by using semi-structured interviews and a focus group, this study was able to distinguish areas of explicit importance to patients. In line with findings from patient satisfaction research ${ }^{29,30}$, this study shows that respondents generally report positive experiences with the care at the outpatient clinic. During the semi-structured interviews, most of their remarks concerned the following dimensions: information, communication and education; coordination and integration of care; and respect for patients'values, preferences and expressed needs. Within these dimensions, they attached great importance to three types of action: building a trusting relationship with one's doctor; open and honest communication by all employees of the outpatient department; and the doctor conveying a calm attitude that gives a patient room to express preferences or ask questions. These findings corroborate those of a recent study that explores patient experience with PCC at an in-patient acute care medical unit ${ }^{31}$. In addition, our respondents said that being assertive was an important factor in having a positive experience at the outpatient department. This too is in line with results in the literature ${ }^{31}$.

If respondents reported negative experiences at the outpatient department, they mostly concerned one of the same three dimensions. In general, respondents thought that the dimensions physical comfort and involvement of family and friends were of secondary significance. They either had satisfying experiences with regard to these latter dimensions or thought a particular dimension did not contribute significantly to their perception of a high quality of care. Opinions on the dimension emotional support -- relieving fear and anxiety seemed to differ among the respondents. Although they do attach importance to this dimension, they seemed unsure whether attention for emotional support is the responsibility of the provider or the patient. In contrast, a study evaluating the predictability of the dimensions of PCC for overall quality of care in a general hospital population found the dimension emotional support to have the greatest influence ${ }^{12}$. This might imply that the relative importance of a dimension for patients differs between certain groups of patients.

A second important insight gained from this study is that patients with complex health problems differ from patients with relatively simple health problems in the way they attribute relative 
significance to the dimensions. Patients with high ADL restrictive, complex symptoms reported more negative experiences in the dimension coordination and integration of care and seemed to be more affected by the problems. For this group, seeing the same doctor and reducing waiting times in the process seemed to have greater importance than for patients with low ADL restrictive, relatively simple symptoms.

The third and probably most important insight is that a qualitative research design can lead to a deeper understanding of the needs and preferences of a patient population. An additional benefit is that patients are able to contribute to the process of improvement by offer suggestions on how negative experiences can be dealt with in the future. This could be very helpful when thinking about redesigning healthcare delivery towards more PCC. The challenges in shifting from a provider-centred care system to PCC are well known and described in the literature ${ }^{10,22,31}$ ${ }^{34}$. This study adds to that literature by demonstrating that exploring patients' experiences and preferences in depth offers empirical grounds for thinking about and shifting towards PCC.

\section{Limitations}

The results of this study are comparable to those from similar studies in other settings or carried out with different research methods. Therefore, the results might be self-evident to some healthcare professionals. However, this is the first attempt to explore the experiences and preferences of ORL patients in depth using qualitative research methods in order to create a scientific basis for the delivery of patient-centred care for this group of patients. In addition, this study shows that qualitative research can contribute to a sound understanding of patients' experiences and preferences. As this study was a first attempt to understand patients' perceptions of PCC as defined by the loM, some interesting differences between subgroups of patients - patients with hearing loss vs. balance disturbance, for instance - are not examined here. It might be useful to investigate those differences in more detail in future research.

Using qualitative research methods can place some limitations on the generalizability and validity of the findings. The COREQ guidelines specify 32 items divided into three domains. These domains are said to influence the methodological robustness of qualitative studies ${ }^{24}$. The first concerns the research team and reflexivity. For this study, the interviews and focus group were led by a trained and experienced researcher who had no treatment relationship with the respondents that could influence their answers. The second domain concerns the study design, which influences the quality of the outcomes. For this study, the framework of PCC set forth by the loM was used as a guide, and maximum variation sampling was applied to select respondents. The sample size of the study population might be considered as medium. Nonetheless, using maximum variation sampling to select information-rich cases, narrowing the scope of the study by focusing on the six dimensions of PCC, and conducting a focus group with the same respondents (i.e., repeated measures) decreases the need for a large sample ${ }^{35}$. The reasons for non-response are unknown. Therefore, the findings might be positively biased; i.e., respondents with positive experiences might have been more willing to participate. It should 
be noted that this study was carried out in an academic hospital, and findings can be influenced by context-specific factors. The third domain covers the analysis and findings. Holding a focus group interview to verify and deepen the findings of the semi-structured interviews was seen as a means to overcome the tendency to give socially acceptable answers and to increase validity.

\section{CONCLUSION}

This report provides in-depth insight into patients' experiences and preferences at an ORL outpatient clinic. By using qualitative research methods to evaluate patient experiences, a distinction is made between the relative significance of dimensions of PCC for this patient population and differences are shown in attributed significance between subgroups of patients. The results imply that patients' perceptions of PCC might differ on the basis of characteristics such as disease complexity. Therefore, the findings underline the need to assess patients' perceptions of PCC carefully before initiating improvements in the field of PCC. In addition, although the methods used in this study led to an in-depth exploration of patients' perceptions of PCC at an ORL outpatient clinic, combining a range of research methods will lead to the most robust measure of PCC. Finally, the results of this study imply that patients are able to participate in projects to enhance quality by suggesting ideas for improvements and by explaining their perspectives. When aiming to shift towards more PCC, it is therefore worthwhile to consult patients before planning improvement projects. 


\section{REFERENCES}

1. De Silva D. Helping measure person-centred care. A review of evidence about commonly used approaches and tools used to help measure person-centred care. London: the Health Foundation, 2014

2. Institute of Medicine. Crossing the Quality Chasm: A New Health System for the 21st Century. Washington, DC: National Academies Press, 2001.

3. de Boer D, Delnoij D, Rademakers J. The importance of patient-centered care for various patient groups. Patient Educ Couns. 2013; 90 (3): 405-410.

4. Bauman AE, Fardy HJ, Harris PG. Getting it right: why bother with patient-centred care? Med J Aust. 2003; 179 (5): 253-256.

5. Bertakis KD, Azari R. Patient-centered care is associated with decreased health care utilization. J Am Board Fam Med. 2011; 24 (3): 229-239.

6. Orn M. Impact of incorporating a patient-centered care model. J Nurses Staff Dev. $2010 ; 26$ (4): 164-169.

7. Richards T. Enlist the patients' help. BMJ. 2011 ;343: d5827.

8. Stewart M, Brown JB, Donner A, et al. The impact of patient-centered care on outcomes. J Fam Pract. 2000; 49 (9): 796-804.

9. Hunter R, Carlson E. Finding the fit: patient-centered care. Nurs Manage. 2014; 45 (1): 38-43.

10. Sidani S, Fox M. Patient-centered care: clarification of its specific elements to facilitate interprofessional care. J Interprof Care. 2013; 28(2): 134-41

11. DiGioia A, 3rd, Lorenz H, Greenhouse PK, Bertoty DA, Rocks SD. A patient-centered model to improve metrics without cost increase: viewing all care through the eyes of patients and families. $J$ Nurs Adm. 2010; 40 (12): 540-546.

12. Shulman HM, Sullivan KM, Weiden PL, et al. Chronic graft-versus-host syndrome in man. A long-term clinicopathologic study of 20 Seattle patients. Am J Med. 1980; 69(2): 204-217.

13. Freitag M, Carroll VS. Handoff communication: using failure modes and effects analysis to improve the transition in care process. Qual Manag Health Care. 2011; 20(2): 103-109.

14. Giesen P, Smits M, Huibers L, Grol R, Wensing M. Quality of After-Hours Primary Care in the Netherlands: A Narrative Review. Ann Intern Med. 2011; 155(2): 108-U196.

15. Tsianakas V, Maben J, Wiseman T, et al. Using patients' experiences to identify priorities for quality improvement in breast cancer care: patient narratives, surveys or both? BMC Health Serv Res. 2012; 12: 271 .

16. Coyle J, Williams B. Valuing people as individuals: development of an instrument through a survey of person-centredness in secondary care. J Adv Nurs. 2001; 36(3): 450-459.

17. Little P, Everitt H, Williamson I, et al. Preferences of patients for patient centred approach to consultation in primary care: observational study. BMJ. 2001; 322(7284):468-472. 
18. Stewart M. Towards a global definition of patient centred care. BMJ. 2001; 322(7284): 444-445.

19. Boss EF, Thompson RE. Patient satisfaction in otolaryngology: Can academic institutions compete? Laryngoscope. 2012; 122(5): 1000-1009.

20. Boss EF, Thompson RE. Patient experience in the pediatric otolaryngology clinic: does the teaching setting influence parent satisfaction? Int J Pediatr Otorhinolaryngol. 2013; 77(1): 59-64.

21. Nieman CL, Benke JR, Ishman SL, Smith DF, Boss EF. Whose experience is measured? A pilot study of patient satisfaction demographics in pediatric otolaryngology. Laryngoscope. 2014; 124(1): 290-294.

22. Davidoff RA, Hackman JC. Hyperpolarization of frog primary afferent fibres caused by activation of a sodium pump. J Physiol. 1980; 302: 297-309.

23. Hackman BW. Brunel's half-sovereign. Lancet. 1980; 1(8176): 1038.

24. Tong A, Sainsbury P, Craig J. Consolidated criteria for reporting qualitative research (COREQ): a 32-item checklist for interviews and focus groups. Int J Qual Health C. 2007; 19(6): 349-357.

25. Patton M. Qualitative evaluation and research methods. Beverly Hills, CA: Sage, 1990.

26. Fereday J, Muir-Cochrane E. Demonstrating Rigor Using Thematic Analysis: A Hybrid Approach of Inductive and Deductive Coding and Theme Development. International Journal of Qualitative Methods. 2006; 5(1): 80-92.

27. Miles M, Huberman M. Qualitative data analysis: An expanded sourcebook (2nd ed.). Thousand Oaks, CA: Sage, 1994.

28. QSR International. NVivo qualitative data analysis software. Version 9 [computer program]. 2010.

29. Crow R, Gage H, Hampson S, et al. The measurement of satisfaction with healthcare: implications for practice from a systematic review of the literature. Health Technol Assess. 2002; 6(32): 1-244.

30. Kleefstra S, Kool R, Zandbelt L, de Haes J. An instrument assessing patient satisfaction with day care in hospitals. BMC Health Serv Res. 2012; 12: 125.

31. Ferguson LM, Ward H, Card S, Sheppard S, McMurtry J. Putting the 'patient' back into patientcentred care: an education perspective. Nurse Educ Pract. 2013; 13(4): 283-287.

32. Epstein RM, Street RL, Jr. The values and value of patient-centered care. Ann Fam Med. 2011; 9(2): 100-103.

33. van der Eijk M, Nijhuis FA, Faber MJ, Bloem BR. Moving from physician-centered care towards patient-centered care for Parkinson's disease patients. Parkinsonism Relat Disord. 2013; 19(11): 923-927.

34. Mclnnes DK. Don't forget patients in patient-centered care. Health Aff. 2010; 29(11): 2127; author reply 2128.

35. Morse JM. Determining sample size. Qual Health Res. 2000; 10(1): 3-5. 



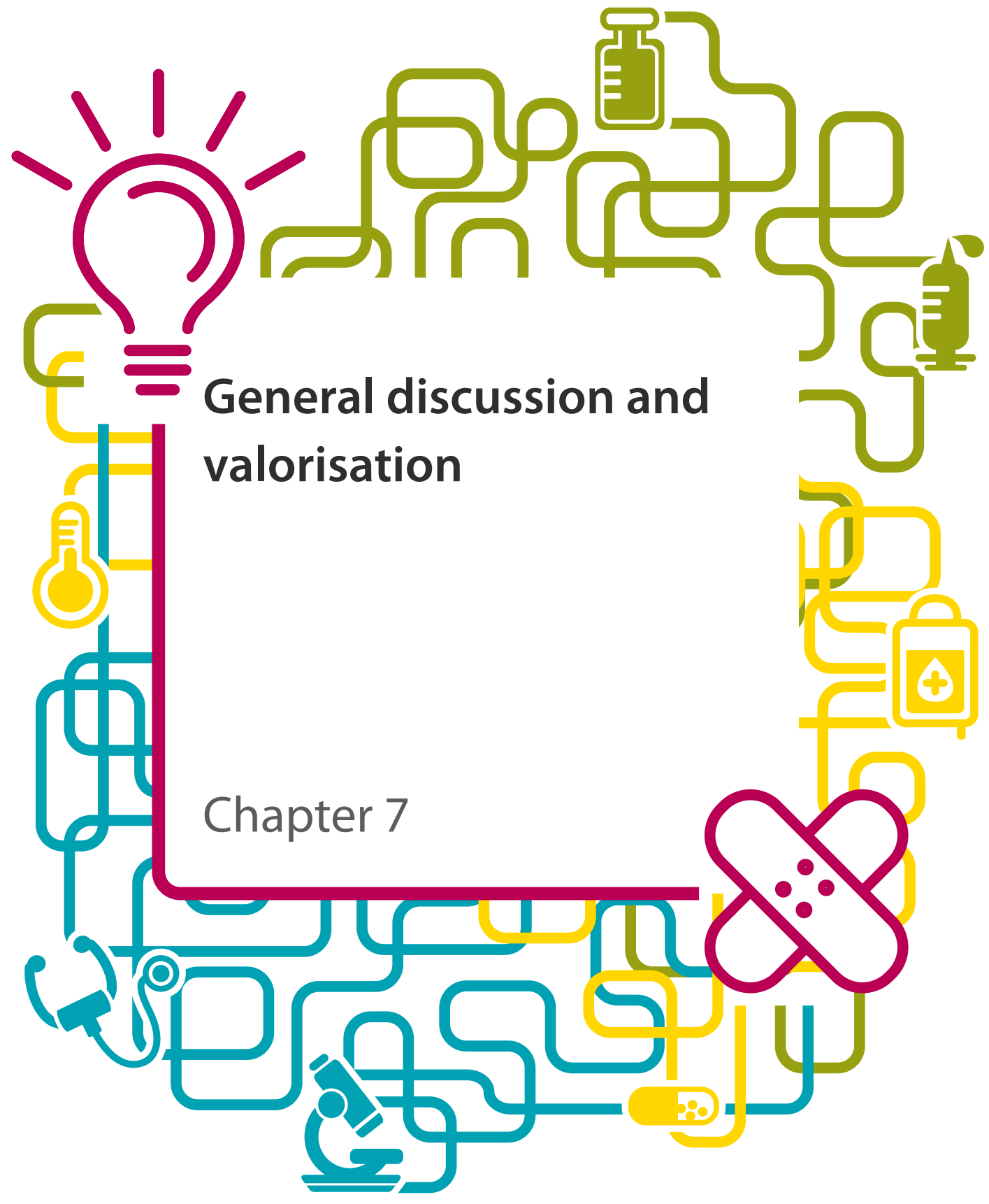


The overall aim of the research presented in this dissertation was to explore if, why and how redesign initiatives were successful. These questions were posed both within an acute and elective care setting. This final Chapter summarizes the main findings by means of these three questions and places them in the context of similar studies, discusses the methodological considerations and societal impact of the findings and provides recommendations for science and practice.

\section{MAIN FINDINGS}

The main conclusions of the studies described in this dissertation can be described as follows:

- In Chapter 2 it was concluded that the influence of process redesign on quality of care could not be established based on the literature as currently available. None of the included articles in the review reported on all its aspects. A wide range of outcome measures were used, and research methods were limited.

- In Chapter 3, literature on organizational routines showed to be useful to explain the occurrence of interprofessional communication failures in the acute care chains. The results of this study also implied that it is worthwhile to further explore the role of organizational routines on interprofessional communication in acute care chains to develop a solution for communication failures.

- The findings of Chapter 4 show that organizational factors play an important role in the success or failure of redesigning a communication process in acute care chains. Organizational routines can hamper implementation of a redesign if it differs too much from the routines of care providers involved. Besides focusing on provider characteristics in the implementation of a redesigned process, specific attention should be paid to unlearning existing organizational routines.

- The findings of Chapter 5 suggest that the implementation of Lean Thinking at an otorhinolaryngology (ORL) outpatient clinic led to a reduction of waste and an increased provider satisfaction on communication. Patient satisfaction did not change significantly, however, it cannot be concluded that the intervention did not have an effect on patients' perceptions of quality of care. Other approaches to measure patients' perspectives should be considered.

- Chapter 6 showed that using qualitative research methods allowed to gain a deeper understanding into patients' experiences and preferences at an ORL outpatient clinic. Using such an in depth evaluation can be very useful in shifting towards more patient-centered care (PPC).

The studies described in this dissertation were part of two research projects, and each of the Chapters highlights one or more questions from the conceptual framework (Figure 1).

Chapter 2 primarily focuses on the if-question, by summarizing the current knowledge available in literature on the influence of redesigning processes on quality of care. Chapters 3 and 4 mainly discuss the why-question by addressing root causes for communication failures and 


\section{Evaluating process redesign initiatives in health care}

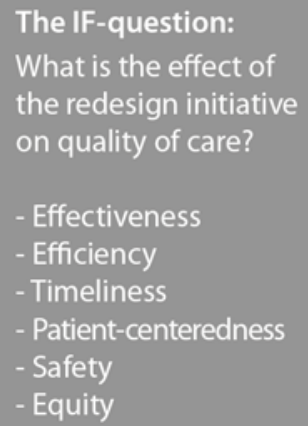

The WHY-question:

Why did the

redesign initiative

work or not?

Facilitators

- Barriers

- Sense of Urgency
The HOW-question:

How did the redesign

initiative affect the

organization?

- Willingness to

change

- Openness

- Feedback Culture

Figure 1 - Conceptual framework

barriers to the implementation of a redesign initiative in acute care chains. Chapter 5 discusses both the if-and how-questions, as it focuses on the effects of Lean implementation on waste (if), patient satisfaction (if), provider satisfaction (how) and organizational culture (how) at an ORL outpatient clinic. Finally, Chapter 6 explores patients' experiences and preferences for ORL outpatient care, thereby addressing the if-question.

\section{ADDRESSING THE IF-QUESTION}

\section{The effects of process redesign on quality of care}

As discussed in Chapter 2, an important goal of redesigning healthcare processes or even systems is to optimize quality of care as delivered to patients ${ }^{2-4}$. For over a decade, redesign initiatives have been undertaken to improve quality of care $^{5}$. Numerous interventions - such as disease management programs, Quality Improvement Collaboratives, Business Process Redesign and Lean Thinking - are tested and implemented in a variety of settings. Despite the efforts, evidence supporting process redesign in health care is limited and inconsistent ${ }^{5}$. Problems with weak study designs, variation of outcome measures and a variety of terminology used to define process redesign hamper the determination of the influence of process redesign on quality of care ${ }^{2,5,6}$. In addition, concerns have been raised that current evaluation studies overestimate the effects of redesign initiatives due to publication bias and the relatively short periods of follow-up ${ }^{6-8}$. In general, process redesign initiatives have the potential to improve quality of care, mostly reported as improvements of effectiveness, efficiency and patient safety. PCC, timeliness and equity of care seem to be neglected in most studies. Attention for PCC is growing however, which may result in more studies evaluating the effect of redesign initiatives on the degree of PCC delivered. Timeliness might partially overlap with efficiency measures and may not be neglected as such in literature, however problems with terminology could be 
responsible for the lack of clear findings on this dimension. The same reasoning holds for equity of care. In general, there is a need to define a robust framework for the evaluation of process redesign initiatives, in which terminology is clarified, research methods are robust and length of follow-up is longer, in order to determine the full effects of redesign initiatives on quality of care ${ }^{2,6-9}$. Unless such a framework is developed and used, comparability and generalizability of research findings is limited, and progress will stay slow and fragmented. Several authors have already made valuable contributions as to how such a framework should look like, but the application of these ideas is lagging behind ${ }^{6,10-13}$. Further on in this Chapter, ideas on this framework resulting from the studies included in this dissertation will be presented.

\section{Exploring patients' experiences and preferences for care delivery}

One of the quality improvement goals as set forward by the loM is to deliver PCC, in which health care is respective and responsive to patients' preferences, needs and values ${ }^{4}$. Achieving such a state of PCC must be founded on insights into the experiences and preferences of patients. One of the findings of Chapter 5 of this dissertation was that a solid insight into experiences and preferences of patients visiting the ORL outpatient clinic was lacking. Exploring patients' experiences and preferences by means of a qualitative study showed to lead to beneficial insights (Chapter 6). In general, patients were able to provide opinions on the care provided to them, and patients were able to make differentiations between several dimensions of PCC. These insights suggest that patients can be valuable partners in redesigning healthcare services around the needs of the patient. In the literature, more examples of patient involvement in the design of evaluation of healthcare processes are known. One of the ways is experience-based co design $(E B C D)$, in which services are co-designed with the patient ${ }^{1,14}$. More specifically, the

1. Setting-up
2. Engaging staff and
gathering experiences
3. Engaging patients/
carers and gathering
experiences
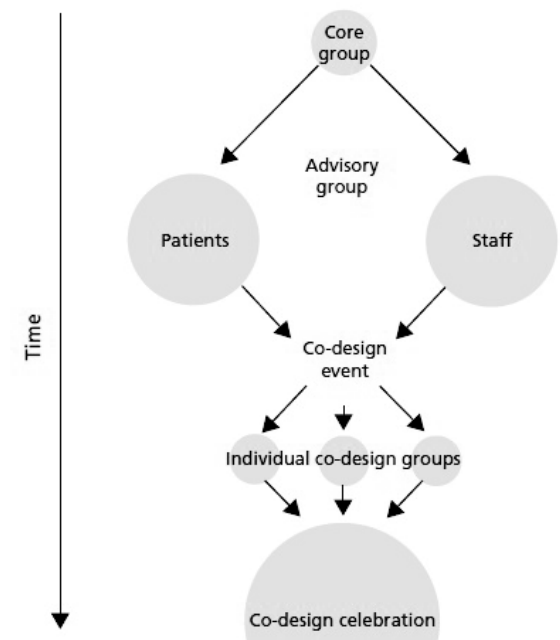

Figure 2 - Six steps of Experienced Based Co Design ${ }^{1}$ 
aim of EBCD is to design experiences, rather than processes in order to improve both patient and provider experiences of health care, thereby improving quality of health care ${ }^{1,14}$. The typical EBCD improvement projects consist of six steps (Figure 2), in which the experiences of both the providers and patients are gathered, brought together, priorities for improvements are set, implemented and eva-luated. This approach has been taken up recently in different countries, leading to sustained improvements in care delivery. In essence, EBCD is not seen as a replacement of existing redesign techniques but rather as an additional perspective that serves to enhance patient-centered health care ${ }^{1,14}$. Since the approach involves rigorous qualitative research techniques it is considered to be time consuming and costly by policymakers in the field however, resulting in a major barrier for its uptake. A recent report investigating the use of accelerated EBCD showed positive findings however, suggesting that methods to overcome this barrier do exist ${ }^{1}$. In addition, one could argue that as using an approach like EBCD will result in improved patient and provider experiences in health care, its associated costs are most likely to be in proportion to its revenues.

\section{Using Lean Thinking to improve quality of care}

Lean Thinking is increasingly applied to healthcare settings with the aim to improve efficiency and optimize care delivery. In general, implementation of Lean shows improvements in the performance of healthcare systems, such as shortened treatment times, reduced waiting times and reduced errors and incidents ${ }^{7,15-21}$. Applying Lean to an ORL outpatient clinic showed promising results, with reduction of transportation, motion and waiting being visible to the employees of the outpatient clinic (Chapter 5). A recent comprehensive review of the literature on Lean suggested that in most cases, Lean led to increased productivity and cost efficiency ${ }^{22}$. In line with the findings as displayed in Chapter 2 of this dissertation however, the authors of this review stress the need for a comprehensive evaluation framework, and the challenge to evaluate the full effects of Lean in health care ${ }^{22}$. The findings of Chapter 5 not only suggest Lean implementation to improve efficiency, it also shows that qualitative research methods can be useful in evaluating the effect of Lean. Using qualitative research methods provided deeper understanding of perceived improvements and can contribute to improved evaluation of Lean implementation. Nevertheless, in order to determine the full effects of Lean, long term comprehensive evaluation using qualitative and quantitative methods should be used $16,23,24$.

\section{ADDRESSING THE WHY-QUESTION}

\section{The influence of organizational routines on process redesign}

Although the concept of organizational routines is rarely applied in healthcare settings, its theoretical background has shown to be useful in understanding difficulties in multidisciplinary cooperation and innovation ${ }^{25-28}$. In Chapter 3 and 4, the concept was used to understand communication failures in acute care chains and implementation barriers of a communication redesign. The concept showed to be a valuable addition to current knowledge in understanding why failures were present in the current process and why implementation of the redesign 
initiative had failed. First, while health professionals increasingly work in a multidisciplinary environment (within or across organizations), organizational routines are often still embedded within organizations or restricted to specific professions ${ }^{29-31}$. The incongruence between the need to closely cooperate with other health professions on the one hand, and organizational routines being mono-professional on the other hand can form a potential threat to effective and efficient cooperation. Since the demand for multidisciplinary, integrated care is rising ${ }^{32-34}$, it seems important to pay attention to changing these organizational routines while redesigning processes in health care. This understanding is confirmed when identifying barriers towards the implementation of a redesign for information transfer and feedback in acute care chains. Next to hampering factors such as top down implementation approaches and the absence of a high sense of urgency for change, implementation of a redesign can also be hampered if the new routine differs too much from the old one (Chapter 4). In this sense, process redesign can be considered to be the alteration of 'old' organizational routines into'new' organizational routines. Successively implementing a redesign requires organizational unlearning of the 'old' routines next to organizational learning of the 'new' routines. While the international literature lacks a clear definition of organizational unlearning, all definitions have in common that unlearning has to do with the elimination of memory. Organizational unlearning involves eliminating beliefs, routines, and physical artifacts ${ }^{35-38}$. Organizational learning and unlearning occur at the same time, as organizational unlearning often involves the replacement of old routines with new ones. Following the concept of eliminating beliefs, routines and physical artifacts however, replacing 'old' routines with 'new' ones will ultimately not suffice and hence will not lead to sustained change. In addition, a recent study on sustainability of healthcare improvement showed that double loop learning - which includes a change in both actions (i.e. routines) and theories and values (i.e. beliefs) - enhances sustained improvements ${ }^{39}$. Therefore, it is very important to emphasize on unlearning beliefs, routines and physical artifacts while learning new beliefs, routines and physical artifacts at the same time. This is likely to lead to improved and sustained results in process redesign.

\section{ADDRESSING THE HOW-QUESTION}

\section{The broader effects of Lean implementation}

As discussed in Chapter 5, besides its effect on operational outcomes, Lean implementation appears to influence employees and the organizational environment as well ${ }^{17}$. The latter factor is receiving little attention in current international literature however. In line with other studies, the findings of the project as carried out for this dissertation suggest that Lean implementation influences providers' perceptions of their organizational environment, such as the willingness to change and the openness among employees at the ORL outpatient clinic (Chapter 5). Even though the available international evidence on Lean implementation suggests improved outcomes on a broad spectrum of outcome measures, some limitations to these findings exist however. First, it seems that the level of Lean implementation in health care is restricted to both implementing Lean as a set of tools rather than an overarching philosophy ${ }^{16,17,19,20,40}$. Second 
implementation of Lean mostly is restricted to individual departments - also applying to Chapter 5 - rather than whole systems ${ }^{19}$. Contrasting to the current state of Lean implementation in health care, the Lean philosophy requires a radical shift in thinking about an organization and its work processes ${ }^{24}$. These statements are underlined by the findings in Chapter 5 , in which respondents stated that they could not oversee the full impact of the Lean project after six months.

Determining the full impact of Lean in healthcare organizations therefore is a matter of long term follow-up, not only including performance measures, but measures on organizational environment and implementation level as well. In line with the findings from Chapter 2, one could argue that the implementation of a whole-system philosophy like Lean, requires holistic evaluations that allow to gain understandings on the effects and the mechanisms through which these effects are established.

\section{COMBINING IF, WHY AND HOW}

The studies in this dissertation addressed several aspects of redesign initiatives in health care. In general, the findings suggest that process redesign initiatives in health care have the potential to improve quality of care and have an impact on organizational aspects such as willingness to change and openness among employees. Asking the if, why and how questions while initiating process redesign in health care is useful to gain a deeper understanding of the implications of redesign initiatives.

Based on current literature, we are unable to define which redesign initiative has the most potential in a particular healthcare setting (Chapter 2). Hence, with the current available literature, concluding if a specific redesign initiative is working, why it works or not and how the initiative will affect the organization is hardly possible. The results of the studies conducted for this dissertation provide more evidence on these aspects and some valuable lessons can be drawn, furthering the current state of evidence. Foremost, the findings in this dissertation form a strong call for an improved evaluation framework for process redesign initiatives in health care. This call is supported by similar studies, reviewing the implementation of initiatives like Lean Thinking ${ }^{7,22}$, Six Sigma ${ }^{16,23}$ and Quality Improvement Collaboratives ${ }^{8}$. Addressing the if, why and how questions in this dissertation also led to the conclusion that the influence of redesign initiatives go beyond quality of care. Instead, process redesign in health care has an effect on performance measures and socio-organizational aspects. These insights provide important knowledge for answering the why question, since changes in routines, values and beliefs are suggested to be of importance in process redesign initiatives. Generally, the results in this dissertation provide a direction for future science and practice. Based on the findings, one could argue that a comprehensive long-term evaluation framework using the three questions as a starting point would further the knowledge on the effects of process redesign in health care. Future directions will be discussed in more detail in the recommendation section of this Chapter. 


\section{METHODOLOGICAL CONSIDERATIONS}

The two projects in this dissertation were carried out by means of participatory action research (PAR), implicating that the researcher plays an active role in the research project. While this type of research might lead to limitations considering objectivity of the research findings, it has also shown to lead to deeper insights into research topics and implications. In this section, the strengths and weaknesses of the methods used will be discussed, and the potential sources of bias in the research findings will be explored.

\section{The value of participatory action research}

The main difference between PAR and more classic designs of research is not the methods used, but the active involvement of people who are affected by the subject under study ${ }^{41}$. An important strength of this type of research is its ability to bridge the gap between research and practice, by emphasizing on characteristics like culture, settings and people rather than on effectiveness alone ${ }^{42}$. In line with this reasoning, Pawson and Tilley ${ }^{43}$ and later on Berwick ${ }^{44}$, argued for realist evaluations of healthcare innovations. In these type of evaluations, mechanisms through which interventions work in the specific context they are implemented in are considered to be responsible for the outcome patterns. Put differently, the outcomes of a redesign initiative are caused by the sum of mechanisms and the intervention itself. The scholars of both PAR and realist evaluation strongly emphasize the weaknesses and incapacity of classic research approaches - like Randomized Clinical Trials and controlled before and after studies - to evaluate the impact of process improvements. These research approaches focus on eliminating factors such as local context or differing mechanisms through which interventions can work in order to reduce bias in research findings. However, such research designs almost never lead to satisfying outcomes in terms of why interventions worked, for whom and in what setting. These questions are however extremely important for policy makers to be answered, creating a knowledge gap between research and practice ${ }^{45}$. The findings in this dissertation are based on PAR and indeed show insights into why a certain redesign was not implemented in a certain setting (Chapter 4), provided a lens through which the existence of communication failures could be explained - thereby unraveling some mechanisms - (Chapter 3), and showed different outcome patterns resulting from the implementation of a lean redesign (Chapter 5 , how it affected the organization in the broader sense). If the research methodology would have been solely focused on determining effectiveness of both redesign interventions, the conclusions would have been limited to the understanding that the implementation of a redesign for information transfer and feedback had failed and that the implementation of lean to an ORL outpatient clinic had been successful in reducing waste. The insights provided by using PAR as a research approach are much richer however, leading to nuanced understandings and providing the ability to formulate more detailed recommendations for both research and practice. 


\section{Validity and reliability of findings}

The use of PAR as a research approach in this dissertation also required the use of mixed methods for data collection in both projects. In the first project - concerning the redesign of information transfer and feedback in acute care chains - semi-structured interviews, questionnaires and focus groups were used as data sources. Combining those data sources led to richer understandings on both the root causes of communication failures and the reasons for implementation failure. In the second project - being the implementation of Lean Thinking at an ORL outpatient clinic - semi-structured interviews, questionnaires and focus groups were used as well. While the impact of the implementation of Lean was measured with semi-structured interviews, patient- and provider satisfaction was measured using questionnaires, and qualitative analysis was used to gain insights into patients' perspectives on patient-centered care. Potential bias was limited by the use of purposive sampling techniques in both projects and the questionnaires used to evaluate patient- and provider satisfaction were based on validated questionnaires and showed good reliability scores. In addition, combining qualitative and quantitative methods enabled us to triangulate data, strengthening validity of findings.

One of the most important characteristics of the findings in this dissertation is the implementation of redesign initiatives in their local context. This might lead to the conclusion that the external validity of findings is limited, however, as PAR and realist evaluation scholars argue, it are these local conditions that are important to acknowledge when evaluating process changes ${ }^{43,44}$. Indeed, it might be that in this particular project organizational routines acted as barriers for implementation (Chapter 4) and this might not hold for other acute care chains in the Netherlands. The value of the findings however, lies in the fact that organizational routines have shown to act as a mechanism through which implementation of the redesign failed. This finding is not limited to the context of the project, but might also hold for other settings. The same reasoning holds for other findings in this dissertation. The direct findings might not be generalizable to other settings, but they indicate mechanisms through which redesigns work or not (i.e. why a redesign initiative works), which is valuable to use in different settings.

As discussed earlier, using purposive sampling techniques enhanced validity of the findings, as did using data triangulation. In the qualitative study (Chapter 6) focus group interviews were used to validate findings from the semi-structured interviews with a sample of the respondents.

Despite the efforts taken to limit potential bias in this dissertation, some limitations remain present and should be taken into consideration when interpreting the findings. First, it is important to stress that the direct findings were related to the research setting and cannot directly be transferred to other settings. Second, while attempting to create solid understandings of the root causes for communication failures, it cannot be guaranteed that factors other than presented in this dissertation have influenced the occurrence of communication failures. The same reasoning holds for presented barriers to implementation of the redesign for information transfer and feedback. Next, the length of follow-up as used in the evaluation of Lean at the ORL outpatient clinic causes a potential underestimation of the effects. In nature, Lean Thinking 
requires a radical change of mindset amongst employees which cannot be expected to be effectuated in one year. Whereas the findings presented in this dissertation might show trends at the ORL outpatient clinic, full effects of Lean implementation should be monitored longitudinally. Publication bias cannot be excluded in general, as there is a tendency to only publish positive results in literature ${ }^{46}$. The results of process redesign initiatives as displayed in Chapter 2 might therefore be overestimated. Next, even though mixed methods were used to evaluate different aspects of redesign initiatives, the specific questionnaires (for both satisfaction research and semi-structured interviews) might not capture the full effects on these measures. The researcher encouraged the respondents to freely elaborate on each topic and asked if there were important issues left that were not discussed earlier on in the interview, thereby creating space for respondents own concerns. In addition, questionnaires were based on earlier validated questionnaires and outcomes were in line with other literature on the same topic.

In both projects, the researcher took an active role in the design, implementation and evaluation of the redesign initiatives. While this is one of the strengths of PAR, is can also potentially weaken the study findings. Personal characteristics of the researcher might have influenced the answers given by the respondents, the way in which implementation activities were carried out and the content of the redesign initiatives. By working in projects groups, training the researcher in holding interviews and regular feedback sessions with a supervision team however, the potential bias accompanying this type of research is kept to a minimum. Finally, while using theories on organizational routines, process typology, implementation barriers, quality of care and patient-centered care enabled structuring of findings, it should be acknowledged that a plethora of terms and factors exist in international literature, describing the same phenomena. It is possible that by restricting the research to these definitions, other factors that might play important roles were left out from the findings.

\section{SOCIETAL IMPACT}

From a societal perspective, the findings of the projects carried out for this dissertation have implications on a national and international level. In this section, the societal impact of the findings presented in this dissertation will be discussed.

\section{Valorisation}

The next two paragraphs will describe how the research conducted for this dissertation is useful both nationally and internationally. The additional value of the research described in this dissertation is presented and its relevance to policymakers, scientists and governments is clarified. In the recommendation section, future plans for practice, education and science are defined.

\section{Quality of care on a national level}

According to the Dutch government, health care should be affordable, accessible and of good quality. Transparency and monitoring of quality of care is being emphasized on by the Dutch government and several bodies exist to enhance or monitor healthcare quality ${ }^{47,48}$. Overall, 
quality of care is monitored by the National Healthcare Institute (In Dutch: Zorginstituut Nederland), emphasizing on measuring patient experiences, enhancing transparency of quality of care and developing standards for quality of $\operatorname{care}^{50}$. In addition, the Health Care Inspectorate (IGZ) enforces quality of health services, prevention measures and medical products by advising the responsible ministers and the application of various measures so that health care providers solely offer 'responsible' care ${ }^{50}$. The Inspectorate investigates and assesses in a conscientious, expert and impartial manner, independent of party politics and unaffected by the current care system ${ }^{50}$. Organized by profession, evidence-based medicine creates an important foundation for high quality care in the Netherlands, as guidelines, protocols and standards of care are being used in care delivery. Finally, quality certificates exist in health care, such as certificates and registers for healthcare organizations, providers, management systems and services ${ }^{51}$. Altogether, quality of health care is strongly being emphasized on by the Dutch government. In addition, the Dutch government recognizes the need for PCC, by emphasizing a transition from systems to persons, thereby creating the need to redesign the organization of healthcare nationally ${ }^{52}$.

Despite the application of research projects on a local level in this dissertation, findings presented are well embedded in the national emphasis on quality of care. The implications of the findings go beyond local application by shedding light on important topics of healthcare quality to address in the future (i.e. effectiveness, efficiency, patient-centeredness, safety, equity and timeliness). The current state of redesigning healthcare services in order to enhance healthcare quality and the methods by which healthcare quality are and should be evaluated and monitored is of paramount importance. In essence, the findings of this dissertation provide policy makers with scientific background to improve the current state of redesign initiatives in health care by addressing important pitfalls in current initiatives.

On a local level, the key findings as presented in this thesis can be used to guide future redesign efforts in the area of Maastricht. As already stated in the introduction, the Maastricht UMC+ strongly focuses on the adoption of an integrated and cohesive approach to health care, acknowledging the changing needs of patients $s^{53-55}$. Redesigning its healthcare services to address the changing needs of patients and achieve the desired level of patient care is an inevitable part of the activities of the Maastricht UMC+. In doing so, lessons can be learned from the efforts described in this dissertation. Most important, the findings suggest that future attempts should be focusing on long-term evaluations of whole-system redesign initiatives, including measures on quality of care (i.e. if the redesign initiative works), mechanisms of change (i.e. why the redesign works) and organizational context (i.e. how the redesign initiative affects the organization).

\section{Quality of care on an international level}

The need to redesign healthcare services in order to increase quality of care is not restricted to the Netherlands, but is acknowledged by most Western countries ${ }^{56-59}$. In line with the international 
literature on process redesign evaluations, the findings in this dissertation too suggest process redesign to positively influence quality of care. The research conducted in light of this dissertation however, adds to existing literature by using different perspectives to evaluate existing healthcare problems (i.e. the concept of organizational routines), in depth exploration of patients' experiences and perspectives with care delivery, evaluating the effects of process redesign (by means of Lean implementation) at an ORL outpatient clinic and systematically reviewing the literature on the effect of process redesign on quality of care. Apart from the specific root causes, implementation barriers, efficiency gains and changes in satisfaction found in this dissertation, a more general message can be formulated. The findings in Chapter 2 led to the understanding that although redesign initiatives have the potential to improve quality of care, we cannot define which redesign initiative has the most potential in what setting. To overcome fragmentation of redesign efforts, we need to build evidence around the three questions posed in this dissertation. More specifically, also internationally, future redesign initiatives should include measures on quality of care (i.e. if the redesign initiative works), mechanisms of change (i.e. why the redesign works) and organizational context (i.e. how the redesign initiative affects the organization). This call for improved evaluations of redesign initiatives closely fits to other efforts made on this topic internationally. The World Health Organization (WHO) has detected important evidence gaps in the delivery of people-centered and integrated health services in a recent interim report ${ }^{59}$. Amongst others, the WHO urges to engage all stakeholders in the development and measurement of people-centered, integrated health services and strongly advises to incorporate quality improvement into health systems reforms ${ }^{59}$. Next, initiated by Berwick ${ }^{56}$ in 2008, scholars on quality improvement are already shifting from only addressing efficacy issues of redesign initiatives towards measuring the so-called 'Triple Aim'. Understanding that the success of quality improvement efforts (such as redesign initiatives) relates to three interdependent goals (i.e. improved experiences of care, improved population health and lower per capita costs) finds audience by policy makers, professionals and scholars around the world. Generally, the impact and success of redesign initiatives go beyond effectiveness and using frameworks like the Triple Aim and the three questions posed in this dissertation can aid further understanding and implementation efforts of redesign initiatives internationally.

\section{RECOMMENDATIONS}

The general message from this dissertation is that better evidence needs to be produced on if redesign initiatives improve quality of care, why redesign initiatives work or not and how redesign initiatives affect the organization or system. Based on this message, recommendations for practice and future research can be made.

\section{Recommendations for practice and education}

The main findings from this dissertation urge policy makers in health care to address the need for quality improvement on a long-term, whole systems manner. Financial incentives need to stimulate long-term redesign initiatives, preferably in whole-system settings. And although a 
whole-system redesign initiative is not likely to be feasible, projects need to move away from redesign initiatives being implemented at departmental level. A first step could be to initiate process redesign for specific populations (such as oncology patients) or in an entire organization (e.g. an entire hospital instead of one outpatient clinic).

In doing so, all stakeholders involved should be aware of the urgency to change, specific goals need to be formulated on all aspects of redesign (i.e. the if, why and how) and financial sources need to stimulate long-term follow-up of the redesign initiative. In essence, policymakers need to specifically focus on the creation and definition of value in health care in general, instead of optimizing specific services ${ }^{13,60}$.

Defining all stakeholders in redesign initiatives goes beyond the scope of this dissertation, the patient is however specifically addressed here. Creating value for the patient can be seen as the overarching goal of healthcare delivery ${ }^{13,60}$, but patients are less frequently involved in process redesigns. Patients' opinions are however of value in such redesign projects. It is therefore recommended to add patients to project groups while undertaking redesign efforts, as is done in experience based co-design (EBCD) projects. Even though such redesign efforts might be timeconsuming at first stage, using EBCD in addition to common redesign techniques add a valuable perspective and will aid redesign projects in reaching their primary goals (i.e. improving quality of care for the patient). Furthermore, as recently evaluated by Locock et al ${ }^{63}$, audio and video archives of patient experiences can be used in more than one project, leading to what they call accelerated EBCD. Therefore, besides the positive associations between patient experiences and health outcomes for a wide range of diseases, investing in EBCD next to common redesign techniques is not necessarily a time-consuming and costly procedure if applied to more than one project - which a whole systems approach implies. In addition, it is important to notice here that EBCD does not replace existing redesign techniques such as Lean Thinking, Six Sigma or BPR. EBCD should be considered as an addition to these techniques.

Project groups concerned with process redesign can consider double loop learning in order to achieve sustained improvements. This double loop learning needs to specifically take into account the unlearning of the 'old' routines (by means of unlearning mechanisms, values and beliefs) while also learning 'new' routines.

Finally, the current developments in health care not only ask for a redesign of its service delivery, healthcare providers require additional skills and a change in mindset as well. Professionals need to be able to closely cooperate with each other in order to deliver multidisciplinary wholesystem care. A committee on Innovation of healthcare professions and education therefore advises to change the existing curriculum of health professions ${ }^{61}$. This dissertation adds to that advice that changing the existing curriculum of medical education is a prerequisite to change existing routines in healthcare and specific attention should be paid to unlearning the existing routines that need to be changed. 


\section{Scientific recommendations}

The call for comprehensive process redesign research can be heard throughout this dissertation. Comprehensive process redesign research can stimulate ongoing efforts in health care by unraveling the black box of process redesign, by stimulating evidence-based redesign implementation and by the uptake of whole-system approaches in process redesign. More specifically, the main message in this dissertation urges scholars in the field of quality improvement to include measures on quality of care (i.e. if the redesign initiative works), mechanisms of change (i.e. why the redesign works) and organizational context (i.e. how the redesign initiative affects the organization) in evaluating redesign initiatives. The interpretation of the if, why and how questions is important in this matter, as uniform evaluations will lead to improved evidencebased redesign initiatives. Therefore, it is also recommended to develop a set of indicators that can be used for the evaluation of redesign initiatives. Multiple initiatives already exist, as the WHO for example currently develops a set of indicators for people-centered and integrated health services ${ }^{59}$. The indicator set should include indicators on experiences of care (e.g. quality of care indicators), population health and costs of care, thereby addressing the importance of the 'Triple Aim' in health care.

The evaluation of redesign initiatives should move away from classic designs such as RCTs and controlled before and after studies and instead focus on using PAR or realist evaluation as an approach. As realist evaluation poses that context and mechanisms together are responsible for outcome patterns, using this type of evaluation will inevitably lead to understandings as to how and why outcomes are achieved, and for whom in which setting. In applying this type of research, it is important to generate a profile of a specific 'PAR-researcher' in order to achieve high quality research. A typical PAR-researcher should have distinct communication skills, is responsive to organizational features and has the capacity to relate and translate science into practice. The role of the researcher in PAR projects should be subject to evaluation during the project as well. Comprehensive evaluation of redesign efforts should carefully consider using longer follow-up periods. Redesign efforts often ask for changes in the mindset of the users, the impact of which can only be determined in the long term. In addition, as redesign projects might use forms of incremental instead of radical change, outcomes can only be expected to present in the long term. Therefore, in order to produce sound evidence, long-term, comprehensive evaluation of redesign projects is necessary. In order to enhance feasibility of long-term evaluation, modeling studies can be used at the outset of redesign initiatives to estimate their effects on certain aspects. It should be carefully noted however, that important contextual factors of redesign initiatives are less suitable for modeling studies and the effects of these studies can therefore be overestimated ${ }^{62,63}$.

Long-term follow up of redesign initiatives can only be effectuated if financial resources stimulate these research designs by developing long-term grants for this type of research. Together, directory boards of healthcare organizations, health insurance companies and other funding agencies like ZonMw and NWO need to reconsider the terms of their research programs 
in order to create a climate for long-term evaluations. This way, skilled PAR-researchers can be retained in redesign initiatives, enabling high quality evaluations of these initiatives.

Finally, in order reach a state of evidence (or impact)-based redesign, the uptake of this type of research in scientific literature needs to be improved. Using structured reporting guidelines such as the SQUIRE guidelines ${ }^{64}$ to report on these initiatives might help researchers to get their studies published and improve reporting of these projects. This recommendation however addresses both the need for improved reporting of redesign initiatives, as well as the need for editors of international peer-reviewed journals to recognize the need to publish these evaluations, regardless of their (positive or negative) outcomes. 


\section{REFERENCES}

1. Locock L, Robert G, Boaz A, et al. Using a national archive of patient experience narratives to promote local patient-centered quality improvement: an ethnographic process evaluation of 'accelerated' experience-based co-design. J Health Serv Res Policy. 2014.

2. Elkhuizen SG, Limburg M, Bakker PJ, Klazinga NS. Evidence-based re-engineering: re-engineering the evidence--a systematic review of the literature on business process redesign (BPR) in hospital care. Int J Health Care Qual Assur Inc Leadersh Health Serv. 2006;19(6-7):477-499.

3. Harmon P. Business Process Change. A manager's guide to improving, redesigning and automating processes. San Francisco, CA: Morgan Kaufmann Publishers; 2003.

4. Committee on Quality of Health Care in America loM. Crossing the Quality Chasm: A New Health System for the 21st Century. Washington, DC: National Academies Press; 2001.

5. Institute of Medicine. Performance Measurement: Accelerating Improvement. Washington, D.C.: Institute of Medicine;2006.

6. Shojania KG, Grimshaw JM. Evidence-based quality improvement: the state of the science. Health Aff (Millwood). 2005;24(1):138-150

7. Mazzocato P, Savage C, Brommels M, Aronsson H, Thor J. Lean thinking in healthcare: a realist review of the literature. Qual Saf Health Care. 2010;19(5):376-382.

8. Schouten LM, Hulscher ME, van Everdingen JJ, Huijsman R, Grol RP. Evidence for the impact of quality improvement collaboratives: systematic review. BMJ. 2008;336(7659):1491-1494.

9. Dimitrov DM, Rumrill PD, Jr. Pretest-posttest designs and measurement of change. Work. 2003;20(2):159-165.

10. Gillam S, Siriwardena AN. Evidence-based healthcare and quality improvement. Qual Prim Care. 2014;22(3):125-132.

11. Siriwardena AN. Understanding quality improvement through social network analysis. Qual Prim Care. 2014;22(3):121-123.

12. Siriwardena AN, Gillam S. Evaluating improvement. Qual Prim Care. 2014;22(2):63-70.

13. Porter ME. A strategy for health care reform--toward a value-based system. $N$ Engl J Med. 2009;361(2):109-112.

14. Bate $P$, Robert $G$. Experience-based design: from redesigning the system around the patient to co-designing services with the patient. Quality \& Safety in Health Care. 2006;15(5):307-310.

15. Collar RM, Shuman AG, Feiner S, et al. Lean management in academic surgery. J Am Coll Surg. 2012;214(6):928-936.

16. DelliFraine JL, Langabeer JR, 2nd, Nembhard IM. Assessing the evidence of Six Sigma and Lean in the health care industry. Qual Manag Health Care. 2010;19(3):211-225.

17. Joosten T, Bongers I, Janssen R. Application of lean thinking to health care: issues and observations. Int J Qual Health Care. 2009;21(5):341-347. 
18. Lin SY, Gavney D, Ishman SL, Cady-Reh J. Use of lean sigma principles in a tertiary care otolaryngology clinic to improve efficiency. Laryngoscope. 2013;123(11):2643-2648.

19. Poksinska B. The Current State of Lean Implementation in Health Care: Literature review. Qual Manag Health Care. 2010;19(4):319-329.

20. Radnor ZJ, Holweg M, Waring J. Lean in healthcare: the unfilled promise? Soc Sci Med. 2012;74(3):364-371.

21. Toussaint JS, Berry LL. The promise of Lean in health care. Mayo Clin Proc. 2013;88(1):74-82.

22. D'Andreamatteo A, lanni L, Lega F, Sargiacomo M. Lean in healthcare: A comprehensive review. Health Policy. 2015.

23. DelliFraine JL, Wang Z, McCaughey D, Langabeer JR, 2nd, Erwin CO. The use of six sigma in health care management: are we using it to its full potential? Qual Manag Health Care. 2013;22(3):210-223.

24. Womack J, Jones D. Lean Thinking. Banish waste and create wealth in you corporation. New york: Free Press; 2003.

25. Novak L, Brooks J, Gadd C, Anders S, Lorenzi N. Mediating the intersections of organizational routines during the introduction of a health IT system. Eur J Inform Syst. 2012;21(5):552-569.

26. Pentland BT, Hrem T, Hillison D. The (N)Ever-Changing World: Stability and Change in Organizational Routines. Organ Sci. 2011;22(6):1369-1383.

27. van Raak A, Paulus A, Cuijpers R, Te Velde C. Problems of integrated palliative care: a Dutch case study of routines and cooperation in the region of Arnhem. Health Place. 2008;14(4):768-778.

28. Edmondson AC, Bohmer RM, Pisano GP. Disrupted routines: Team learning and new technology implementation in hospitals. Admin Sci Quart. 2001;46(4):685-716.

29. Elissen AM, van Raak AJ, Paulus AT. Can we make sense of multidisciplinary co-operation in primary care by considering routines and rules? Health Soc Care Community. 2011;19(1):33-42.

30. van Raak A, Groothuis S, van der Aa R, Limburg M, Vos L. Shifting stroke care from the hospital to the nursing home: explaining the outcomes of a Dutch case. J Eval Clin Pract. 2010;16(6):1203-1208.

31. Van Raak A, Paulus ATG, Groothuis S. Integrated Care Delivery: Process Redesign and the Role of Rules, Routines and Transaction Costs. In: Klein LA, Neumann EL, eds. Integrated Heatlh Care Delivers. New York: Nova Science Publishers; 2008:115-135.

32. OECD. Health at a Glance: Europe 2012. Paris: The Organisation for Economic Co-operation and Development; 2012.

33. Raad voor de Volksgezondheid en Zorg. Perspectief op gezondheid 20/20. Den Haag;2010.

34. Rijksinstituut voor Volksgezondheid en Milieu. Een gezonder Nederland. Volksgezondheid Toekomst Verkenning 2014. Bilthoven: RIVM;2014.

35. Akgun AE, Byrne JC, Lynn GS, Keskin H. Organizational unlearning as changes in beliefs and routines in organizations. J Organ Change Manag. 2007;20(6):794-812.

36. Bouton ME. Context, ambiguity, and unlearning: sources of relapse after behavioral extinction. Biol 
Psychiatry. 2002;52(10):976-986.

37. Rushmer R, Davies HT. Unlearning in health care. Qual Saf Health Care. 2004;13 Suppl 2:ii10-15.

38. Tsang EWK, Zahra SA. Organizational unlearning. Hum Relat. 2008;61(10):1435-1462.

39. Hovlid E, Bukve O, Haug K, Aslaksen AB, von Plessen C. Sustainability of healthcare improvement: what can we learn from learning theory? BMC Health Serv Res. 2012;12.

40. Holden RJ. Lean Thinking in emergency departments: a critical review. Ann Emerg Med. $2011 ; 57(3): 265-278$.

41. Cornwall A, Jewkes R. What is participatory research? Soc Sci Med. 1995;41(12):1667-1676.

42. Cargo M, Mercer SL. The value and challenges of participatory research: strengthening its practice. Annu Rev Public Health. 2008;29:325-350.

43. Pawson R, Tilley N. Realist Evaluation. London: Sage Publication; 1997.

44. Berwick DM. The science of improvement. Jama. 2008;299(10):1182-1184.

45. Jansen MW, Ruwaard D. Making an impact instead of 'publish or perish'. Eur J Public Health. 2012;22(5):613-614.

46. Chalmers I, Glasziou P, Godlee F. All trials must be registered and the results published. Brit Med J. $2013 ; 346$

47. Maarse JA, Ruwaard D, Spreeuwenberg C. The governance of quality management in dutch health care: new developments and strategic challenges. Qual Manag Health Care. 2013;22(3):236-247.

48. Schippers E, van Rijn M. Gezamenlijke agenda VWS 'Van systemen naar mensen'. Den Haag: Ministerie van Volksgezondheid, Welzijn en Sport; 2013.

49. Zorginstituut Nederland. Toetsingskader kwaliteitsstandaarden en meetinstrumenten. Den Haag: Zorginstituut Nederland;2014.

50. Inspectie voor de Gezondheidszorg. Jaarbeeld 2013. Den Haag: Inspectie voor de Gezondheidszorg. Ministerie van Volksgezondheid, Welzijn en Sport.;2014.

51. Huntink H, van Oostenbrugge L. Keurmerken, Certificaten en kwaliteitsverklaringen in de zorg. Den Haag: Nictiz \& Kwaliteitsinstituut; 2014.

52. Ministerie van Volksgezondheid, Welzijn en Sport. De maatschappij verandert. Verandert de zorg mee? Den Haag: Ministerie van Volksgezondheid Welzijn en Sport;2014.

53. Maastricht UMC+. Better health in a changing world. Maastricht: Maastricht UMC+; 2012.

54. Maastricht UMC+. Jaarverslag 2012. Maastricht: Maastricht UMC+;2012.

55. Maastricht UMC+. Gezond Leven. Maastricht: Maastricht UMC+;2015.

56. Berwick DM, Nolan TW, Whittington J. The triple aim: care, health, and cost. Health Aff (Millwood). 2008;27(3):759-769.

57. Fuchs VR, Emanuel EJ. Health care reform: Why? What? When? Health Aff (Millwood). 2005;24(6):1399-1414. 
58. Institute of Medicine. Crossing the Quality Chasm: A New Health System for the 21st Century. Washington, DC: National Academies Press; 2001.

59. World Health Organization. People-centered and integrated health services: an overview of the evidence. Interim Report. Geneva: World Health Organization;2015.

60. Porter ME. What is value in health care? N Eng/ J Med. 2010;363(26):2477-2481.

61. Commissie Innovatie Zorgberoepen \& Opleidingen. Naarnieuwezorg en zorgberoepen: de contouren. Den Haag: Zorginstituut Nederland;2015.

62. Baines TS, Asch R, Hadfield L, Mason JP, Fletcher S, Kay JM. Towards a theoretical framework for human performance modelling within manufacturing systems design. Simul Model Pract Th. 2005;13(6):486-504.

63. Shub AN, Stonebraker PW. The human impact on supply chains: evaluating the importance of "soft" areas on integration and performance. Supply Chain Manag. 2009;14(1):31-40.

64. Ogrinc G, Mooney SE, Estrada C, et al. The SQUIRE (Standards for QUality Improvement Reporting Excellence) guidelines for quality improvement reporting: explanation and elaboration. Qual Saf Health Care. 2008;17 Suppl 1:113-32. 



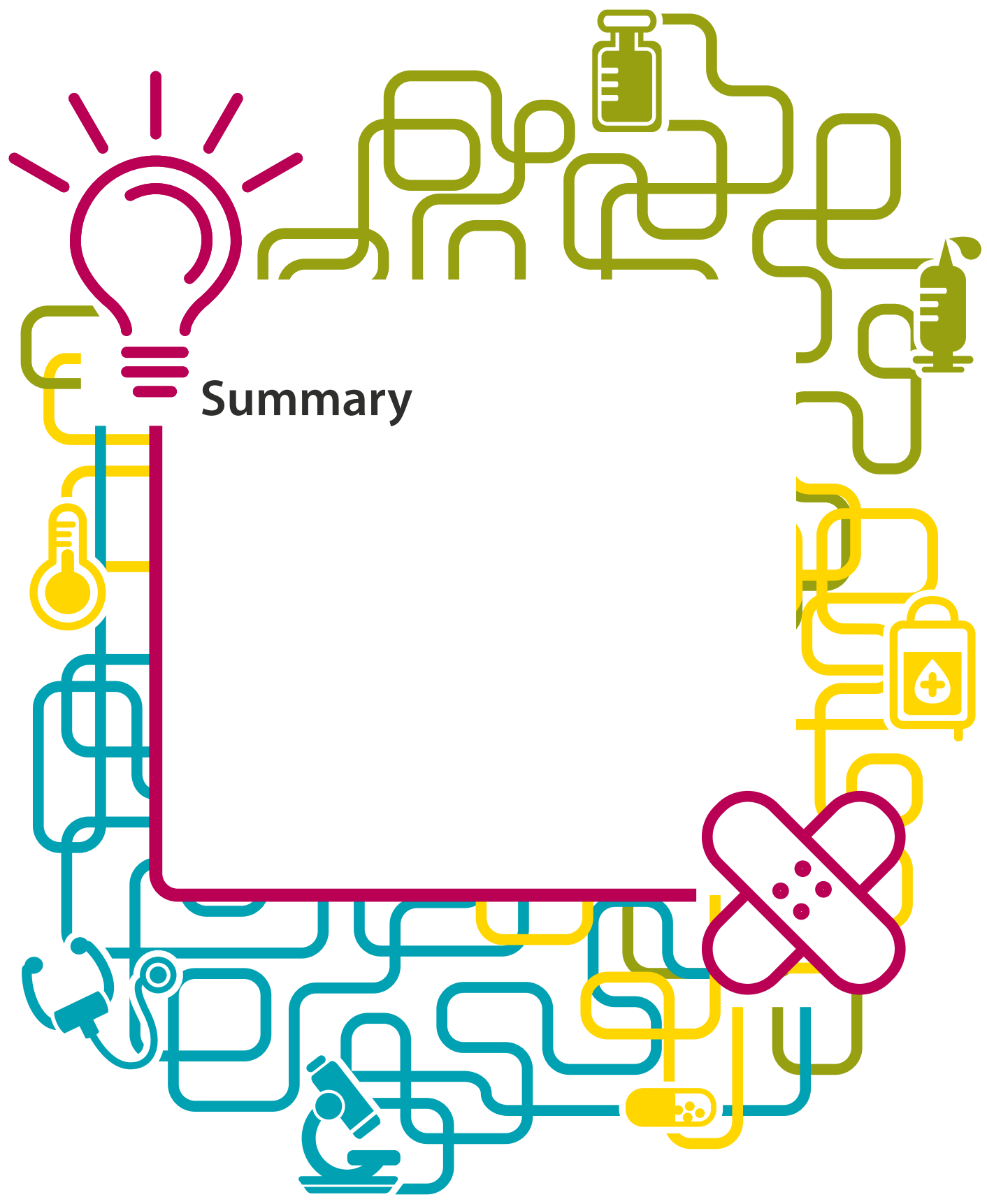




\section{SUMMARY}

The overall aim of the research presented in this dissertation was to explore if, why and how redesign initiatives were successful. These questions were posed both within an acute and elective care setting. Besides understanding the effects of the redesign initiatives on several dimensions of quality of care (i.e. if the redesign had worked or not), attention was being paid to factors contributing to the success or failure of the redesign initiative (i.e. why the redesign had worked or not), and to understand the influence of the redesign initiative on other aspects than quality of care (i.e. how the redesign had worked).

The main focus of Chapter 2 lays on the if-question, by summarizing the current knowledge available in literature on the impact of redesigning processes on quality of care. Pubmed, CINAHL, Web of Science and Business Premier Source were searched for relevant studies published in the last ten years (2004-2014). Identified studies that met the inclusion criteria were independently assessed for reporting quality by three reviewers prior to inclusion in the review, using the SQUIRE checklist. The full impact of process redesign on quality of care could not be determined on the basis of the literature. Studies differed in the type of redesign implemented, study setting, methods used for evaluation, and outcome measures. All types of intervention seemed to improve outcomes in one or more respects. Nonetheless, it is not clear which type of redesign has the most potential in a particular setting. The results underline the need for to further the knowledge on the impact of redesign interventions on the quality of care delivered. In addition, the development of a more uniform methodology to assess the impact of redesign interventions would strengthen the evidence underlying process redesign interventions.

In Chapter 3, the why-question was addressed by exploring the root causes of interprofessional communication failures in acute care chains. Two different explanations were used to gain a deeper insight into these root causes. The first explanation stated that failures depend on the level of integration and standardization of a care process, meaning that the more standardized and integrated a care process is, the fewer failures will occur. The second explanation was based on organizational routines - being repetitive action patterns in organizations. This explanation stated that failures in handover communication were caused by a lack of shared understandings about the organizational communication routine amongst providers, which results in differences in the way a routine is carried out. A multiple case study containing six cases (i.e. acute care chains) was carried out in which semi-structured interviews, physical artifacts and archival records were used for data collection. Based on the level of standardization and integration present in the acute care chains, the six acute care chains could be divided into two categories of care processes. Failures in interprofessional communication occurred in both groups. Communication routines were embedded within organizations and descriptions of communication routines of the acute care chain as a whole - indicating shared understandings - could not be found. Based on the results, failures in interprofessional communication could not exclusively be explained by literature on process typology. Literature on organizational routines was useful to explain the occurrence of interprofessional communication failures in 
the acute care chains. The results of this study imply that it is useful to further explore the role of organizational routines on communication in acute care chains to develop a solution for failures in interprofessional communication.

Chapter 4 defined barriers to the implementation of a redesign of information transfer and feedback in acute care chains, thereby addressing the why-question. As a response to existing communication failures in acute care chains, the information transfer and feedback process was redesigned. Its implementation however failed. In order to understand the barriers to implementation of the redesign, a case study with six cases (i.e. acute care chains), using mixed methods was carried out. Focus group interviews and questionnaires were used for data collection. Respondents reported three sets of barriers for implementation of the redesign: (a) existing routines for information transfer and feedback in organizations within the acute care chain; (b) the implementation method and time period; and (c) the absence of a high 'sense of urgency' amongst providers in the acute care chain which would aid in improving the communication process. The findings of this chapter show that organizational factors play an important role in the success or failure of redesigning a communication process. Organizational routines can hamper implementation of a redesign if it differs too much from the routines of care providers involved. Besides focusing on provider characteristics in the implementation of a redesigned process, specific attention should be paid to unlearning existing organizational routines.

Chapter 5 described the evaluation of a Lean Thinking project at an otorhinolaryngology (ORL) outpatient clinic. This chapter thereby focused on the if and the how-question. The effects of application of Lean to ORL settings as well as the impact on provider satisfaction and organizational culture is underreported. Therefore, an 18-month prospective before and after design using mixed methods was used to determine the impact of Lean on the ORL outpatient clinic. Patient and provider satisfaction was measured by means of a survey and semi structured interviews were held to evaluate the effect of Lean implementation on waste and organizational culture. During the project, 69 issues were written down on the lean board and improvement efforts were realized for 36 inefficiency issues, not all of them concerning a specific type of waste. Considerable improvements were reported by employees for transportation, motion and waiting. Patient satisfaction was high both at baseline and follow-up and did not change significantly. Small effects on provider satisfaction were seen; satisfaction on autonomy and participation decreased significantly and satisfaction on communication increased significantly. The findings of this chapter suggest that the implementation of Lean Thinking at an ORL outpatient clinic led to a reduction of waste and an increased provider satisfaction on communication. Although patient satisfaction did not change significantly, it cannot be concluded that the intervention did not have an effect on patients' perceptions of quality of care. Other approaches to measure patients' perspectives should be considered.

Chapter 6 discussed experiences and preferences of patients visiting an ORL outpatient clinic in the context of patient-centered care (PCC). Herewith the chapter addressed the if-question in more detail. PCC has received considerable attention in the last few decades, however an 
extensive insight from the patient's perspective is lacking. Qualitative research methods were used to measure patients' experiences and preferences, firstly by conducting 22 semi structured interviews, followed by a focus group to verify and deepen the findings. Overall, patients reported positive experiences with PCC at the outpatient department. Dimensions receiving most attention during the interviews and the focus group were information, communication and education, coordination and integration of care, and respect for patients' values and expressed preferences and needs. If patients reported negative experiences it mostly concerned one of these dimensions. The dimensions physical comfort and involvement of family and friends were considered to be of secondary significance to respondents. Patients' opinions about the dimension emotional support - relieving fear and anxiety differed from each other as to whether this was the responsibility of the doctor or the patient. Using qualitative research methods allowed to gain a deeper understanding into patients' experiences and preferences at an ORL outpatient department. Using such an in depth evaluation can be very useful in shifting towards more PPC.

In Chapter 7, the main findings from the research in this dissertation was summarized by addressing the if, why and how-question. The studies in this dissertation touched on several aspects of redesign initiatives in health care. In general, the findings suggest that process redesign initiatives in health care have the potential to improve quality of care and have an impact on organizational aspects such as willingness to change and openness. Asking the if, why and how questions while initiating process redesign in health care seems useful to gain a deeper understanding of the implications of redesign initiatives. The main recommendations of this dissertations therefore are to; (a) develop an evaluation framework which can be used to assess the full impact of process redesign initiatives, (b) include the patient in process redesign initiatives as a partner, for example by using the Experience Based Co-Design template, (c) incorporate double-loop learning in the implementation of redesign initiatives to increase the chances on successful implementation, and (d) improve the uptake of this type of research projects in scientific literature in order to stimulate evidence-based redesign in health care. 



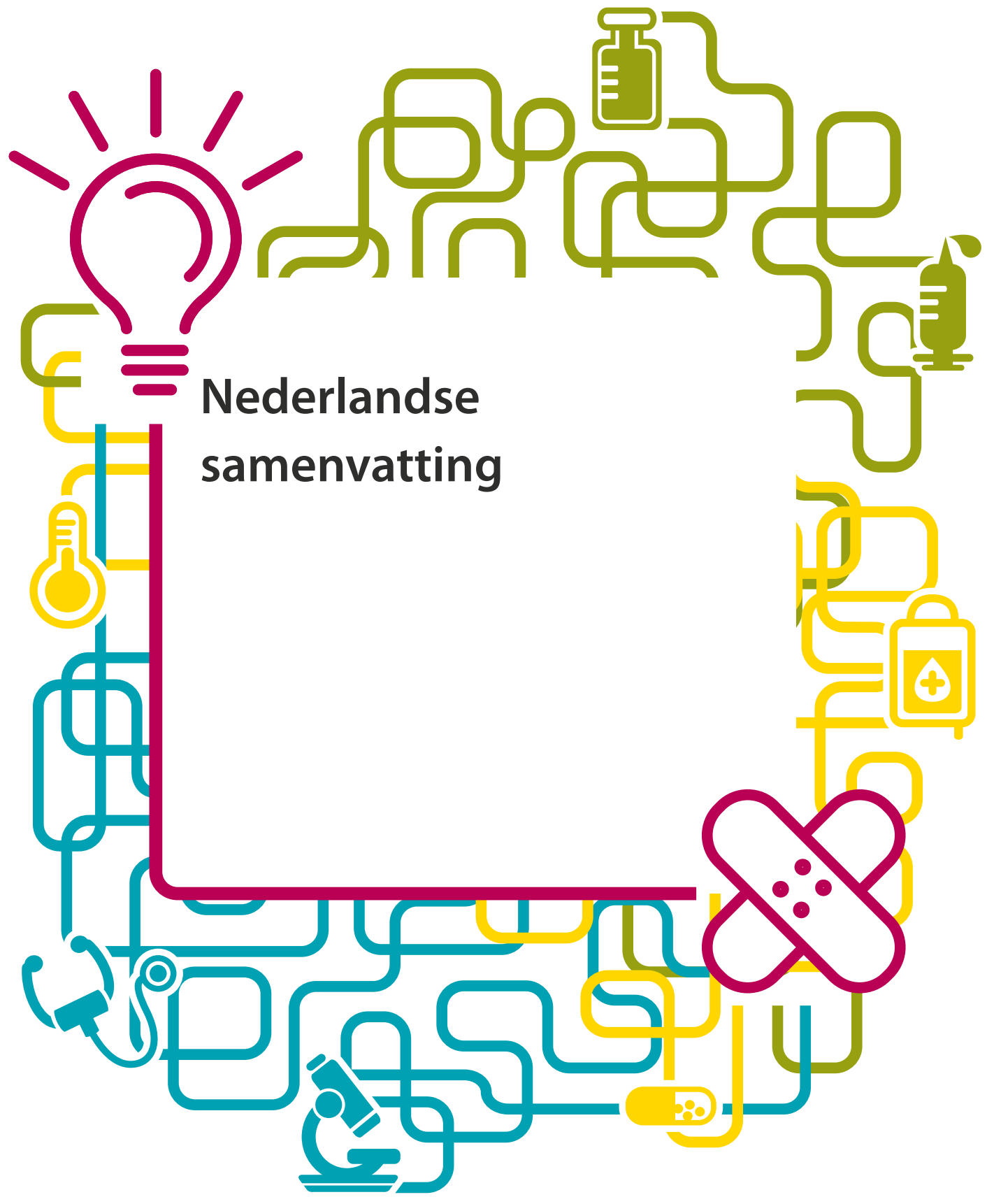




\section{NEDERLANDSE SAMENVATTING}

De centrale doelstelling van het onderzoek in dit proefschrift was om te beschrijven of, waarom en hoe herontwerp initiatieven succesvol waren. Deze vragen werden zowel in een acute alsook in een electieve setting gesteld. Naast het onderzoeken van de effecten van het herontwerpen van zorgprocessen op de kwaliteit van zorg (of het herontwerp had gewerkt of niet), werd ook aandacht besteed aan de factoren die een bijdrage hadden geleverd aan het succes of falen van het herontwerp (waarom het herontwerp had gewerkt of niet) en aan de invloed van het herontwerp op andere organisatorische aspecten dan kwaliteit van zorg (hoe het herontwerp had gewerkt of niet).

In Hoofdstuk 2 werd aandacht besteed aan de of-vraagstelling, door de huidige kennis op het gebied van de effecten van het herontwerpen van zorgprocessen op de geleverde kwaliteit van zorg te onderzoeken. Dit werd bepaald middels een systematische beoordeling van de internationale wetenschappelijke literatuur over dit onderwerp. De databases Pubmed, CINAHL, Web of Science en Business Premier Source werden doorzocht op relevante studies die in de afgelopen tien jaar gepubliceerd werden (2004-2014). Studies die aan de inclusiecriteria voldeden werden door 3 onderzoekers onafhankelijk beoordeeld op de kwaliteit van rapporteren van bevindingen, door middel van het gebruik van de SQUIRE richtlijnen. Het algemeen effect van het herontwerpen van zorgprocessen op de kwaliteit van de geleverde zorg kon op basis van de beschikbare literatuur niet bepaald worden. Studies verschilden in het type herontwerp dat geïmplementeerd werd, studie setting, gebruikte methoden voor evaluatie en uitkomstmaten. Alle herontwerp typen lieten verbetering op één of meerdere aspecten van kwaliteit van zorg zien. Het is echter niet mogelijk om te bepalen type herontwerp de meeste kans van slagen heeft in een bepaalde setting. Daarnaast is het van belang om een meer uniforme methodologie te ontwikkelen om het herontwerpen van zorgprocessen te evalueren, om zo tot sterkere bewijskracht te komen.

In Hoofdstuk 3 stond de waarom-vraag centraal, door de achterliggende oorzaken van knelpunten in interprofessionele communicatie in acute zorgketens te onderzoeken. Hiertoe werden twee verschillende benaderingen gebruikt, elk met een andere invalshoek om de knelpunten te analyseren. De eerste benadering was gebaseerd op literatuur over proceseigenschappen, waarbij gesteld werd dat hoe meer gestandaardiseerd en geïntegreerd een proces is, hoe minder knelpunten zich zullen voordoen. De tweede benadering was gebaseerd op literatuur over organisatieroutines, die omschreven kunnen worden als herhaalde actiepatronen die een belangrijke rol spelen in organisaties. Deze benadering stelde dat knelpunten in communicatie veroorzaakt worden door het ontbreken van een gezamenlijk begrip over de routine, waardoor verschillen ontstaan in de uitvoering van de routine. Een meervoudige case studie met zes casussen (acute zorgketens) werd uitgevoerd, waarbij semi gestructureerde interviews, zogenaamde 'physical artifacts' en archief materiaal gebruikt werd als dataverzameling. Gebaseerd op de mate van integratie en standaardisatie konden de acute 
zorgketens in twee type processen worden verdeeld. Knelpunten in interprofessionele communicatie werden in beide groepen gevonden. Organisatieroutines werden binnen organisaties beschreven en een beschrijving van de communicatie routine op het niveau van de zorgketen als geheel bleef achterwege - duidend op de afwezigheid van een gezamenlijk begrip van de routines. Op basis van de resultaten konden de bestaande knelpunten niet uitsluitend verklaard worden met behulp van proceseigenschappen. Literatuur over organisatieroutines toonde wel behulpzaam in het verklaren van knelpunten. De resultaten die in dit hoofdstuk beschreven zijn suggereren daarom dat het zinnig is om naast bestaande verklaringsmechanismen, ook de rol organisatie routines in communicatie in acute zorgketens verder te onderzoeken en uit te diepen.

In Hoofdstuk 4 werd de waarom-vraag besproken door barrières voor de implementatie van een herontwerp van informatieoverdracht en feedback in acute zorgketens te beschrijven. Hoewel dit herontwerp werd ontwikkeld om bestaande knelpunten in de informatieoverdracht en feedback aan te pakken, mislukte de implementatie ervan. Respondenten die participeerden in de studie gaven 3 groepen van barrières aan, te weten (a) bestaande routines voor informatieoverdracht en feedback binnen organisaties in de acute zorgketens, (b) de implementatie methode en het tijdstip van implementatie en (c) de afwezigheid van een hoge 'sense of urgency' onder zorgverleners in de acute zorgketens. De bevindingen in dit hoofdstuk indiceren dat organisatiefactoren een belangrijke rol spelen in het slagen of mislukken van de implementatie van een herontwerp - in dit geval van een communicatie proces. Organisatieroutines kunnen een belemmerende factor zijn als het herontwerp teveel afwijkt van de huidige manier van werken bij zorgverleners. Hiertoe is het belangrijk om niet alleen aandacht te hebben voor het aanleren van nieuwe werkwijzen, maar ook voor het afleren van bestaande routines.

In Hoofdstuk $\mathbf{5}$ wordt de evaluatie van een Lean Thinking project op een polikliniek Keel-Neusen Oorheelkunde (KNO) beschreven. Hierbij werd zowel de of als de hoe-vraag besproken. De toepassing van Lean wordt regelmatig geassocieerd met positieve uitkomsten, maar over het effect bij KNO-poliklinieken en de effecten op medewerkertevredenheid en organisatiecultuur is minder goed beschreven. Om deze reden werd een prospectieve voor en na studie met een duur van 18 maanden uitgevoerd waarbij gemixte methoden (d.w.z. zowel kwantitatief als kwalitatief) gebruikt werden voor dataverzameling. Patiënttevredenheid en medewerkertevredenheid werden gemeten aan de hand van vragenlijsten en semigestructureerde interviews werden gebruikt om de effecten van Lean op de mate van verspilling en op organisatiecultuur te bepalen. Gedurende het project werden 69 punten van inefficiëntie gemeld op een daarvoor bedoeld Leanbord, waarbij niet in alle gevallen sprake was van een specifiek type van verspilling. Voor 36 van deze punten werd verbetering gerealiseerd. Medewerkers rapporteerden aanzienlijke verbetering voor transport, verplaatsing en wachten tijdens de nameting. Patiënttevredenheid scoorde zowel bij voor- als nameting hoog en een significante verandering werd niet gezien. Er werden kleine significante verschillen waargenomen op het gebied van 
medewerkertevredenheid, waarbij de tevredenheid over autonomie en participatie daalde en de tevredenheid over communicatie significant verbeterde. Ook rapporteerden medewerkers veranderingen op het gebied van organisatiecultuur, en werd een toename van de veranderbereidheid en de openheid op de afdeling gerapporteerd. De bevindingen suggereren dat de implementatie van Lean op een polikliniek KNO tot een afname van verspilling heeft geleid, waarbij patiënttevredenheid onveranderd is gebleven en kleine significante verschillen werden waargenomen op het gebied van medewerkertevredenheid. Daarbij moet opgemerkt worden dat hoewel de implementatie van Lean niet tot zichtbare verandering op het gebied van patiënttevredenheid heeft geleid, het niet te zeggen is of er geen effect te vinden is voor de patiënt. Daartoe zouden andere methoden ingezet moeten worden om de perspectieven van de patiënt te evalueren.

In Hoofdstuk 6 stonden de ervaringen en voorkeuren van patiënten die de polikliniek KNO bezochten centraal, waarmee gedetailleerd op de of-vraag werd ingegaan. Dit werd gedaan in de context van patiënt-gecentreerde zorg, een onderwerp dat de laatste jaren in toenemende mate aandacht krijgt in de internationale literatuur. Een verdiepend inzicht in de perspectieven van de patiënt mist echter nog. Er werd gebruik gemaakt van kwalitatieve onderzoeksmethoden om de ervaringen en voorkeuren van patiënten in beeld te brengen. In eerste instantie werden 22 semigestructureerde interviews met patiënten afgenomen, waarna de bevindingen uit deze interviews gecontroleerd en verdiept werden in een focusgroep met patiënten. Patiënten rapporteerden in het algemeen positieve ervaringen met patiënt-gecentreerde zorg op de polikliniek. De dimensies informatie, communicatie en scholing, coördinatie en integratie van zorg en respect voor de geuite waarden en voorkeuren van patiënten kregen de meeste aandacht tijdens de interviews. Als patiënten negatieve ervaringen melden betrof het meestal één van deze dimensies. De dimensies fysiek comfort en betrokkenheid van familie en vrienden werden van minder belang bevonden door de patiënten. De meningen van patiënten over de dimensie emotionele ondersteuning waren verdeeld, waarbij patiënten vooral verschillende meningen hadden over de vraag of dit onder de verantwoordelijkheid van de zorgverlener of de patiënt valt. Door gebruik te maken van kwalitatieve onderzoeksmethoden was het mogelijk om een meer diepgaand inzicht te krijgen in de ervaringen en voorkeuren van patiënten. Een dergelijke evaluatie toonde zich nuttig voor de kanteling naar meer patiënt-gecentreerde zorg.

In Hoofdstuk 7 staan de voornaamste bevindingen uit dit proefschrift weergegeven, door de of, waarom en hoe-vraag te bespreken. De studies uit dit proefschrift bespraken verscheidene aspecten van het herontwerpen van zorgprocessen in de gezondheidszorg. In het algemeen kan gezegd worden dat het herontwerpen van zorgprocessen potentieel tot verbetering op het gebied van kwaliteit van zorg leidt. Daarbij heeft het herontwerpen van zorgprocessen ook invloed op de organisatiecultuur, bijvoorbeeld door het beïnvloeden van de veranderbereidheid en de openheid binnen een organisatie. Het stellen van de of, waarom en hoe-vraag bij herontwerp projecten lijkt zinnig om verdiepende inzichten te krijgen in de werking en 
implicaties van een herontwerp. De voornaamste aanbevelingen uit dit proefschrift zijn daarom ook om (a) een evaluatie raamwerk te creëren dat gebruikt kan worden om de volledige impact van herontwerp projecten te bepalen, (b) de patiënt vanaf het begin te betrekken bij het herontwerpen van zorgprocessen, bijvoorbeeld door'Experience Based Co-Design'te gebruiken, (c) het zogenaamde 'double loop' leren te gebruiken bij het implementeren van herontwerpen, om de kans op succes te vergroten en (d) het publiceren van dit type onderzoek in wetenschappelijke vakbladen te verhogen, om zo evidence-based herontwerp in de gezondheidszorg te stimuleren. 



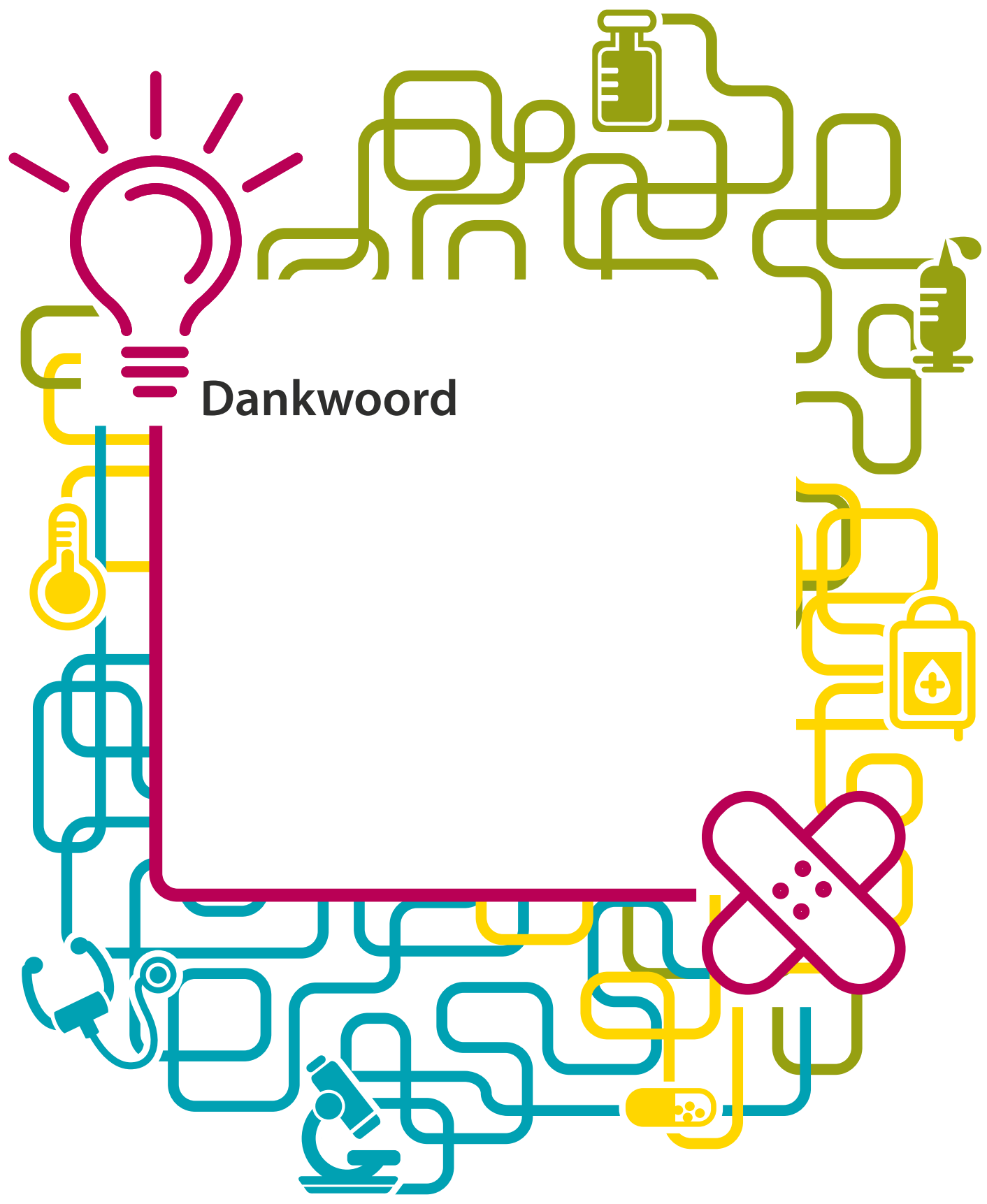




\section{DANKWOORD}

Hoeveel mensen je kent die een bijdrage leveren aan je proefschrift ervaar je pas als je ze in een dankwoord wil benoemen. Een ieder die een bijdrage - klein of groot - heeft geleverd aan de totstandkoming van dit proefschrift wil ik uiteraard hartelijk bedanken. Van al die mensen die dank toekomt, wil ik een aantal mensen in het bijzonder bedanken:

Op de eerste plek gaat mijn dank uit naar alle respondenten (zorgverleners en patiënten) die hebben deelgenomen aan de twee onderzoeksprojecten die de onderlegger van dit proefschrift zijn. Zonder jullie was het niet mogelijk geweest ook maar iets op papier te zetten, daarvoor mijn grote dank!

Mijn promotieteam bestaande uit Prof. dr. Dirk Ruwaard, Prof. dr. Bert Vrijhoef, Prof. dr. Bernd Kremer en dr. Arno van Raak. Prof dr. Ruwaard, beste Dirk, In 2010 werd je hoofd van de vakgroep Health Services Research en niet veel later werd je mijn eerste promotor. Ik heb je al die tijd ervaren als iemand die altijd geïnteresseerd in de mens achter je medewerker - en promovendus. Veel dank voor jouw betrokkenheid bij mijn werk, maar ook zeker voor je interesse in mij. Het heeft me op vele momenten geholpen. Prof. dr. Vrijhoef, beste Bert. Of het nu ging om het stimuleren tot het ingaan van een promotietraject of om het creëren van rust om te schrijven, jij lijkt altijd wel raad te weten en me van goede adviezen kunnen voorzien. Dat kan gebeuren in Maastricht, Singapore, het ziekenhuis van Leuven (onder bijzondere omstandigheden), via telefoon of skype. Het maakt eigenlijk niet uit, elk gesprek bood me mogelijkheden om weer verder te gaan en als ik je nodig had was je gewoon "binnen bereik". Mijn grote dank hiervoor! Prof. dr. Kremer, beste Bernd, richting het einde van mijn promotietraject werd duidelijk dat mijn onderzoek uitgevoerd op de poli KNO een substantieel deel zou gaan uitmaken van mijn proefschrift en werd jouw rol in mijn begeleiding steeds groter. Ik ben blij dat je destijds onderdeel bent geworden van mijn promotieteam! De samenwerking heb ik altijd als prettig ervaren. Ik heb mede daardoor een fantastische tijd gehad bij KNO, mijn dank daarvoor! Dr. Van Raak, beste Arno, een gesprek met jou over een onderzoek waarvoor je financiering had gekregen was de aanstichter van dit alles, alleen al daarvoor mijn dank. We zijn samen begonnen aan dit avontuur en gaandeweg het traject werd het begeleidingsteam uitgebreid. Jij hebt daar altijd een belangrijke rol in gespeeld en je was nooit te beroerd om me gedegen commentaren te geven op stukken (zelfs op de digitale manier!). Dank voor alle gesprekken, adviezen en hulp die je me tijdens dit traject hebt gegeven!

De leden van de beoordelingscommissie, prof. dr. Hans Maarse, prof. dr. Frits van Merode, prof. dr. Harm Haak, prof. dr. Henri Marres en prof. dr. Gert Westert wil ik hartelijk bedanken voor hun inspanningen om mijn proefschrift te beoordelen.

Leden van de projectgroep Spoedzorg, prof. dr. Peter Brink, dr. Inge Duimel, dr. Marielle Kroese, drs. Philo Logister, Dick Nagelhout en dr. Erica Posma, dank voor de ruimte die jullie me gaven om dit project uit te voeren en voor ondersteuning van mijn werkzaamheden op allerlei manieren. Dr. Duimel, lieve Inge, in het bijzonder wil ik jou hier bedanken. Jouw betrokkenheid hield niet 
op bij dit project, je bleef me volgen gedurende mijn promotietraject en daarna, maar ook privé. Ik vind je mailtjes altijd weer leuk om te lezen en we moesten een lunch maar periodiek terug laten komen! Ook alle overige medewerkers van de spoedzorgketens in regio Limburg wil ik hartelijk danken voor hun medewerking aan het project, via interviews, observaties, deelname aan implementatie bijeenkomsten of andere activiteiten.

Leden van de projectgroep Prosecco, dr. Lucien Anteunis, drs. Jan Wouter Brunings, Suzanne Mooi, dr. Sietze Reitsma, drs. Diane Smit en alle werknemers van afdeling KNO, het Audiologisch Centrum en de afdeling Evenwicht, heel veel dank voor jullie inzet! Prosecco was voor mij een geweldig project en ik heb jullie vertrouwen en betrokkenheid hierbij zeer gewaardeerd. Ik heb me dankzij jullie allemaal, en in het bijzonder dankzij Suzanne en Jan Wouter, heel erg welkom gevoeld.

De co-auteurs van mijn artikelen, dr. Inge Houkes, dr. Geert Willem Huismans, Jeroen Bisschop en Kerstin Grube, hartelijk dank voor jullie bijdrage aan het werk in dit proefschrift.

Mijn collega's bij Opera, Jessica, Nicole, Wendy, Mitchel, Koen, Tara, Dennis, Ellen en Judith. Bedankt voor jullie warme welkom op mijn nieuwe plek, voor het geduldig beantwoorden van mijn vele vragen, me op weg helpen als het me aan ervaring ontbreekt en jullie oprechte interesse in elkaar. Ik ben op een hele fijne werkplek terecht gekomen! Een bijzonder dankjewel daarvoor aan Mitchel (geen Ipad, maar nu wel eeuwige roem;))

Mijn collega's bij HSR, jullie zijn met teveel om op te noemen, maar ik wil jullie hier toch bedanken voor de gezellige jaren die ik heb beleefd op de vakgroep. In het bijzonder wil ik Suus, Rietje, Joanna en Brigitte bedanken. Jullie waren in verschillende fases zeer betrokken bij mijn wel en wee en dat was geweldig! Brigitte, dank voor al je secure regelwerk aan het einde van dit traject! Het maakte het allemaal een stuk makkelijker voor me. Ik ga jullie ontzettend missen!

Mijn lieve, lieve kamergenootjes: dr. Cindy Noben, dr. Reina de Kinderen en dr. Arianne Elissen. Wat hebben we toch allemaal niet meegemaakt in kamer 0.044 ! Zonder jullie was dit promotietraject echt lang zo leuk niet geweest en daarvoor ben ik jullie eeuwig dankbaar! De afgelopen jaren zijn zeer vruchtbare jaren geweest in kamer 0.044; twee huwelijken, een huwelijksaanzoek, 3 baby's (bijna 4!) en 4 proefschriften zijn daar een goede afspiegeling van. Ik denk dat je wel kan stellen dat we bijzonder gedreven inwoners van de kamer waren! dr. Noben, lieve Cindy, jij stort je vol enthousiasme op nieuwe projecten en weet er altijd een leuke draai aan te geven. Ook zit je nooit verlegen om een beetje humor en is er altijd wat te beleven met jou op de kamer! Dr. De Kinderen, lieve Reina, dinsdagen en woensdag - en soms ineens een andere dag van de week, heel verwarrend allemaal! Met jou erbij zijn ze gezellig en lekker druk! Je hebt altijd een luisterend oor, bent begripvol en geïnteresseerd. Ook nooit bang om je mening ergens over te geven, heerlijk. Bovendien kan je echt ontzettend lekker koken en heb ik genoten van de maaltijden in Budel! Daar kom ik nog eens voor terug. Dr. Elissen, lieve Arianne, een dramatische rit met de trein naar Den Haag was de aanzet tot jouw aanwezigheid op onze kamer wat een goede zet was dat! Praten met jou over mijn onderzoek, twijfels of wat dan ook was altijd 
fijn. En dan niet te vergeten onze congres tripjes, wat een succes was dat! En dan uiteindelijk samen met een dikke buik, een groot geheim project met veel stiekeme pret! $\mathrm{Na}$ al die dingen was de conclusie: nu moeten we eraan geloven, naast collega's zijn we nu toch echt vrienden geworden. Ik hoop dat we elkaar ondanks de afstand veel blijven zien! En ik ben heel erg blij dat jij mijn paranimf wil zijn!

Maastricht Madness, Lotte, Geoffrey, Laura, Danny, Magali, Idske, Ralph, Bo, Laurens, Roel, Chiel, Linferd \& Liina: sinds 2003 vormen we een bijzonder gemêleerd gezelschap. Inmiddels is iedereen uitgewaaid en is er nog een enkeling in Maastricht te vinden. Niettemin vinden we elkaar elk jaar weer terug voor een weekend in de Ardennen. Wat een feest is dat toch elk jaar weer! Ik hoop dat er nog vele jaren volgen. Lieve Lot, wat kan er toch een hoop gebeuren in een mensenleven. Ik ben blij dat we elkaar ondanks drukte toch blijven vinden, je bent een waardevolle vriendin voor me! Lieve Laura, je bent altijd betrokken en geïnteresseerd en staat voor me klaar. Ik geniet van onbezorgde middagen in het zonnetje met de kinderen om ons heen! Samen met jouw Danny en Magali ben je een waardevolle aanvulling op mijn leven. En ik ben blij dat je me wil steunen door vandaag mij paranimf te zijn! Lieve Ids, het creatieve brein in onze groep en altijd in voor gezelligheid, maar ook altijd geïnteresseerd in ons wel en wee. Heel veel dank ook voor het ontwerpen van de omslag, ik ben er heel erg blij mee! Lieve Chiel, hoewel woorden soms echt te kort schieten, raken wij eigenlijk nooit uitgepraat. Je weet hoe ik onze gesprekken waardeer en hoe trots ik op je ben. Nu staat dat ook mooi nog eens op papier! Dank voor je openheid, alle warme gesprekken en je interesse in mijn (ons) leven! Lieve Prick, je bent echt een vaste waarde in de groep, wil graag dat we allemaal bij elkaar zijn en blijven (ook na 1uur's nachts!). Dankjewel voor je warmte in de groep. Lieve Roel, wat moesten we nu zonder jouw entertainment in de groep? Onze gangmaker, zeker ook in voor serieuze gesprekken, maar immer het feestbeest! Lieve Linferd, als we elkaar zien is het altijd goed en dat is ontzettend fijn! My dearest Liina, your interest in our lives is truly honest and much appreciated by us!

Lieve Marielle, vanaf de vierde klas van de middelbare school vormen wij toch een wat bijzonder duo. Van samen Lingo kijken en kindersurprise eten in de tussenuren naar samen naar de kinderboerderij met onze kleine mannen, daar zitten een heleboel mooie herinneringen tussen! Ik koester ze en ik hoop dat er nog vele bij mogen komen.

Lieve familie Leezer, Jan, Willy, Tom, Yvonne, Bart en Evelien. Jullie vallen eigenlijk ook in de categorie familie bij ons en dat is fijn! Bedankt voor jullie betrokkenheid en interesse in ons wel en wee, maar zeker ook voor de fijne momenten samen, in Nederland of Flayosc (en binnenkort ook in Robertville?). Sven en Chris kunnen zich geen betere 'Bonpa' en 'Bonma' wensen. Lieve Tom, je bent een geweldige vriend en ik vind de momenten dat je weer bij ons bent altijd om van te genieten! Laten we snel naar die vierkantshoeve gaan zoeken, waar die dan ook moet staan :).

Mijn lieve schoonfamilie. Hoewel het soms misschien wel onduidelijk was waar ik me mee bezig houd, vind ik het leuk en fijn om in Ewijk / Nijmegen te zijn en over heel andere dingen te praten! 
Lieve Tobias, Inge, Elise, Guido en Eva, wat een mooi gezelschap (schoon)broers en (schoon) zussen vormen we toch! Ik geniet van de momenten dat we samen zijn, kunnen lachen maar ook onze zorgen kunnen delen met elkaar. Dank voor jullie grote interesse in mijn werk en jullie aanmoedigingen in dit traject, maar zeker ook privé! Zonder was dit zeker weten allemaal niet gelukt!

Lieve, lieve papa en Gusta, mijn dank richting jullie is eigenlijk niet goed op papier te zetten, toch doe ik een poging. Dank voor de oneindige hoeveelheid fijne gesprekken die we hebben gevoerd, voor jullie interesse in mijn (ons) leven en voor het feit dat jullie er altijd voor mij (en ons) zijn! Heel letterlijk heb ik dat gevoeld en dat voelt ontzettend goed.

Mijn laatste woorden van dank gaan uit naar mijn drie liefste mannen: Joost, Sven en Chris.

Lieve, lieve, lieve Joost!. Van jouw passie, gedrevenheid en doorzettingsvermogen heb ik veel kunnen leren. Ook nadat je het gevoel had dat je wereld was ingestort vond je weer de weg terug, ik heb er veel respect voor. Je bent mijn rots in de branding en mijn steun en toeverlaat waarvan ik weet dat je er altijd zal zijn en je altijd achter me zal staan. Alleen daardoor is een mens in staat prestaties te leveren. De helft van de credits voor dit boekje zijn daarmee dus voor jou! Wat ben ik blij dat ik mijn leven met jou kan delen, laten we nog veel mooie geschiedenis samen maken!

Lieve, lieve, lieve Sven en Chris! Als allerlaatst zijn jullie aan de beurt, dat is een bijzondere plek en toont aan hoe bijzonder jullie voor mij zijn. Door jouw geboorte Sven besef ik me dat gewoon zijn eigenlijk heel bijzonder is; zo klein als je was leerde jij mij die grote levensles. Je hebt je helemaal in het begin in Leuven al een sterke en stoere jongen getoond en dat ben je nog steeds. En jij Chris bevestigde die levensles alleen maar. Met jou hebben we er een echte levensgenieter bij gekregen en dat helpt om zwaardere dagen weer licht te maken. Ik geniet intens als ik zie hoe jullie je ontwikkelen en steeds meer van de wereld ontdekken. Daarin ontdek ik ook telkens weer een stukje van de wereld met jullie mee en dat houd me scherp. En jullie mogen het nooit vergeten: ik houd van jullie, tot aan de zon en terug! 



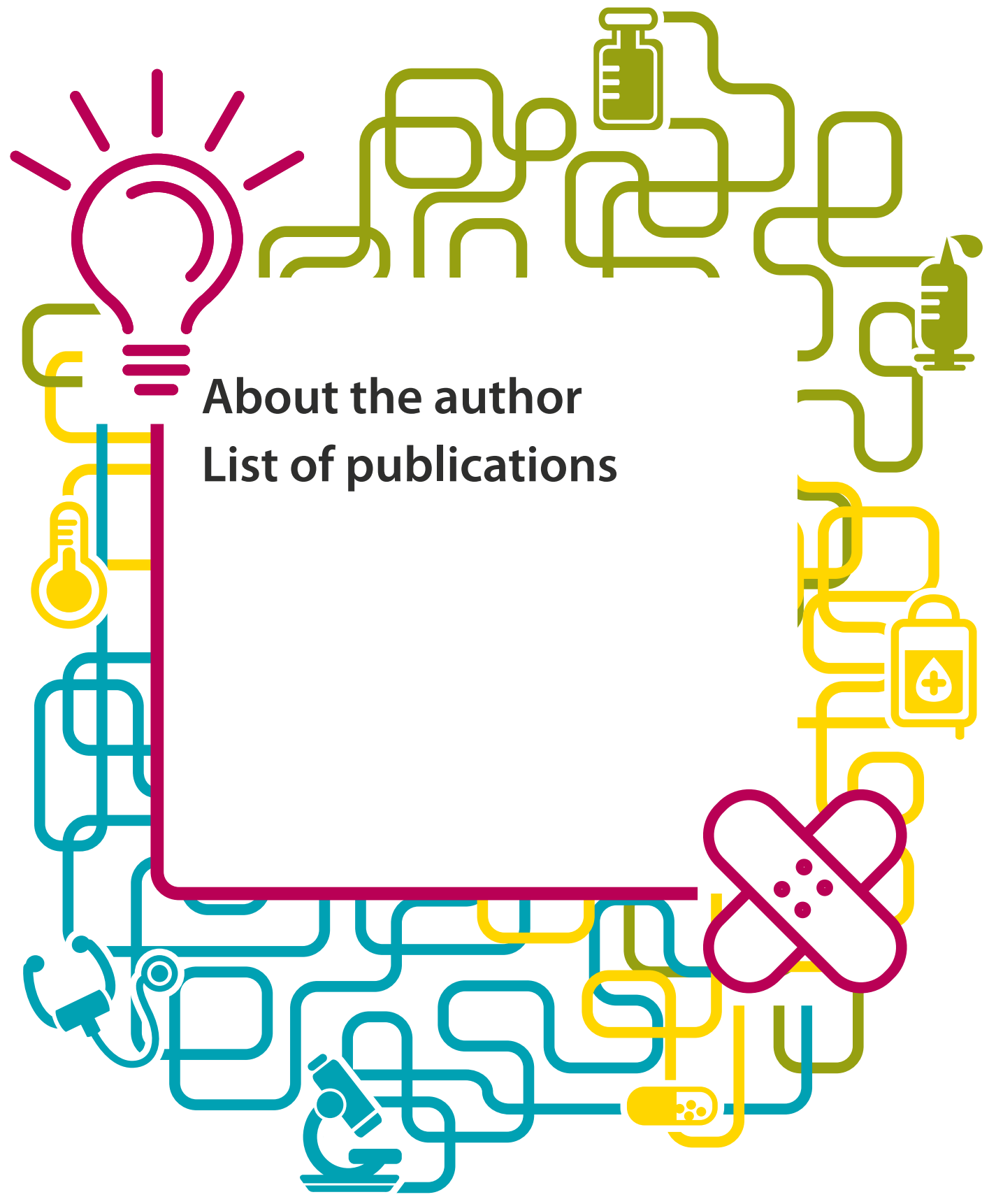




\section{ABOUT THE AUTHOR}

Janneke van Leijen-Zeelenberg holds a master degree in Health Policy, Economics and Management (HPEM) from Maastricht University, the Netherlands. She graduated in August 2008. Ever since her graduation, Janneke especially interested in operational excellence in health care. After graduation, Janneke worked on the development of a care pathway at the MUMC+ and started to work for the Comprehensive Cancer Center Limburg. Her work concentrated on quality improvements in the care provided to palliative patients. From November 2009 till June 2015, Janneke worked at the department of Health Services Research as a PhD-student. Her research

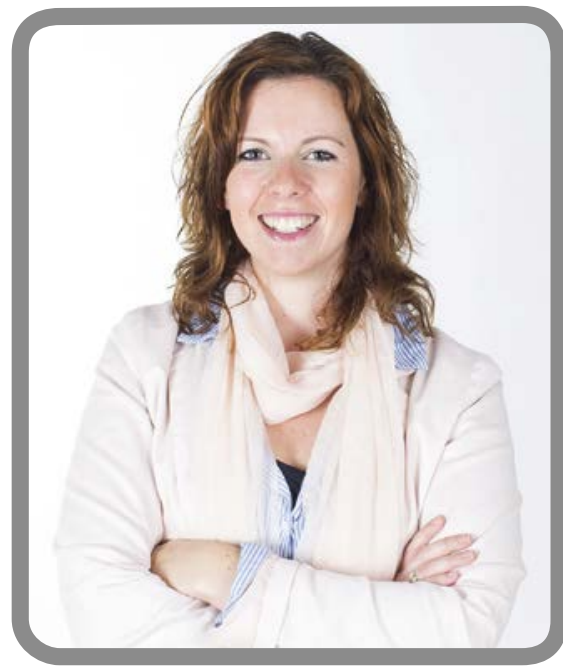
focussed the effect of redesigning healthcare processes on the quality of care provided to patients. She executed research projects in acute care - studying the communication process in acute care chains - and at the Otorhinolaryngology outpatient clinic - studying the effect of changes made by Lean Six Sigma methods in the care processes. Topics of special interest to Janneke are operational excellence, patient-centered care, changing organizational routines and incorporating patient experiences in redesigning healthcare processes.

Currently, Janneke is working as medior consultant at Opera Consultancy \& Implementatie. Here too Janneke focuses on helping healthcare organizations to optimize their care processes to increase efficiency and to succeed to deliver high quality of care. 


\section{LIST OF PUBLICATIONS}

\section{International Publications}

van Leijen-Zeelenberg, JE., van Raak, AJA., Duimel-Peeters, IGP., Kroese, MEAL., Brink, P., Ruwaard, D., Vrijhoef, HJM., (2014) Barriers to implementation of a redesign of information transfer and feedback in acute care: results from a multiple case study. BMC Health Services Research 2014 14:149.

van Leijen-Zeelenberg, JE., van Raak, AJA., Ruwaard, D., Vrijhoef, HJM., (2013) Barriers to implementation of a redesign of information transfer and feedback in acute care: results from a multiple case study. International Journal of Integrated Care; WCIC Conference Supplement; volume 13 URN:NBN:NL:UI:10-1-115997

van Leijen-Zeelenberg, JE., van Raak, AJA., Duimel-Peeters, IGP., Kroese, MEAL., Brink, P., Ruwaard, D., Vrijhoef, HJM., (2015) Interprofessional communication failures in acute care chains: how can we identify the causes? Journal of Interprofessional Care 2015, 29; 4, 320-330

Van Leijen-Zeelenberg, JE., Huismans, GW., Brunings, JW., van Raak, AJA., Vrijhoef, HJM., Ruwaard, D., Kremer, B., (2015) Experiences and preferences of patients visiting an Otorhinolaryngology outpatient clinic: a qualitative study. Health Expectations 2015 (epub ahead of print)

Van Leijen-Zeelenberg, JE., Brunings, JW., Houkes, I., van Raak, AJA., Vrijhoef, HJM., Ruwaard, D., Kremer, B., (2015) Using Lean Thinking principles at an Otorhinolaryngology outpatient clinic to improve patient care. The Laringoscope, accepted for publication

\section{Submitted Publications}

Van Leijen-Zeelenberg, JE., Elissen, AMJ., Grube, K., van Raak, AJA., Vrijhoef, HJM., Kremer, B., Ruwaard, D., The influence of redesigning healthcare processes on the quality of care: a systematic review

\section{Reports}

Zeelenberg, J., van Raak, A., Vrijhoef, B., Duimel-Peeters, I., Kroese, M., Logister, P., Posma, E., Slangen, E., Nagelhout, D., Brink, P., (2011) Eindrapportage Ontwikkelproject. Verbetering van de communicatie en feedback in ketens voor spoedzorg door invoering van een model voor informatieoverdracht en feedback.

\section{National Publications}

van Leijen-Zeelenberg, J.E., (2012) Uitkomsten en aanbevelingen vanuit het project "verbetering van communicatie en feedback in spoedzorgketens". Netwerk Acute Zorg Limburg: Nieuwsbrief juli 2012 


\section{Conferences}

Oral Presentation: "The influence of redesigning care processes on quality of care. A systematic review" at the 2015 International Conference on Integrated Care, 25-27 March 2015, Edinburgh Oral Presentation: "Barriers to the implementation of a communication redesign in acute care chain. Results of a multiple case study" at the 2013 World Conference on Integrated Care, 7-9 November 2013, Singapore.

Oral Presentation: "Increasing efficiency of an Otorhinolaryngology outpatient clinic" at the 2013 EHMA Conference, 26-28 June 2013, Milano, Italy.

Oral Presentation: "Zorglijnontwikkeling Hoofdhals Oncologie" at the Hoofd-Hals symposium, November 24th 2009, Sittard, The Netherlands

Poster Presentation: "Information transfer in acute care chains: Can routines explain the bottlenecks? On the Sixth Mediterranean Emergency Medicine Congress (MEMC VI) 10-14 September 2011, Kos, Greece 

